\title{
Spent Nuclear Fuel Project Multi-Year Work Plan WBS \#1.4.1
}

Date Published

March 1997

Prepared for the U.S. Department of Energy Assistant Secretary for Environmental Management 
IECAL Disclamer

This report wes prepered as en eccount of work eponeored by en egoney of the United States Govornment. Neither the

United States Government nor eny egenoy thereof, nor eny of their employese, nor any of their contrecton, abeontrectore or their employees, mekes eny warrenty, exprese of implied. of exeumes any loged lisbility or recponsibility for the

eceurecy, completenese, or any third party's use or the reaults of weh use of any imformetion, epperatus, product, or proceses diecloeed, of represents that its ues would not infringe privetaly owned righte. Reforence horein to eny epecific commeroid product, process, or eenvioe by trede neme, tredernerk, menufecturer, or otherwiee, does not neceseerily conetitute or imply ite ondorsement, recormmendation, or fovoring by the United Stetes Government or any epency thereof of its contrectore or eubcontrectors. The wiows end opinione of wuthore expreseed herein do not necesenerily atate of reflect those of the United Stetes Government or any epency thereof.

This report hes been reproduced from the beet aveileble copy. Aveilable in paper copy and microfiche.

Availeble to the U.S. Department of Energy

and tis contrectore from

U.S. Depertment of Eneroy

Office of Scientific and Technical information (OST)

P.O. Box 62

Oak Pidge, TN 37831

(615) 576-8401

Printed in the Unined Soute of America

DISCLM-3.CHP $(1-91)$ 


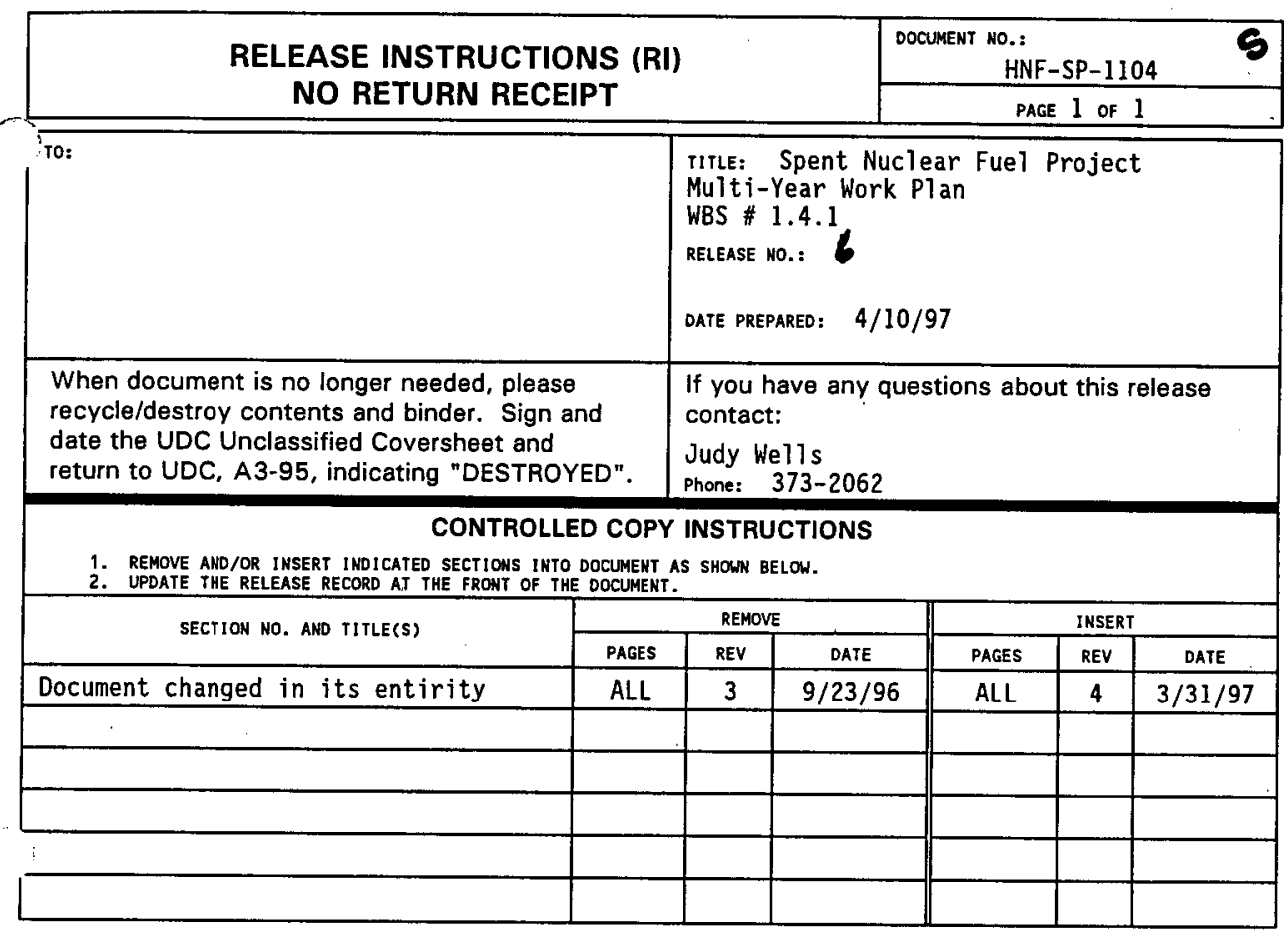

IMPLEMENTATION NOTICE 


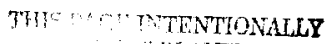

i . "BLANK 


\section{INFORMATION RELEASE REQUEST \\ PUBLIC DOCUMENT CHANGE/REVISION \\ (Short Form No. 1)}

1. Base Document ID Number: HNF-SP-1104

2. Base Document Title:

Spent Nuclear Fuel Project

Multi-Year Work Plan

WBS \#1.4.1

3. Change/Revision Number: Rev 4

4. Change/Revision Date: $3 / 31 / 97$

5. Unclassified Category: ${ }^{*}$ UC -

6. Budget \& Reporting Code: ${ }^{*}$ B\&R -

I verify this change/revision to the base document indicated above:

- Complies with the provisions of WHC-CM-3-4

- Contains no Limited-Use information

- Contains no classified or Applied Technology references

- Does not change the intent or meaning of the base document

- And, the base document itself is approved for public release.

7. Responsible Manager: Jeff L. Denning

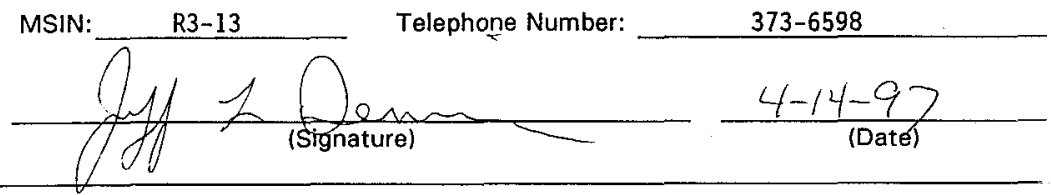

8. Information Release Administration Specialist: * *

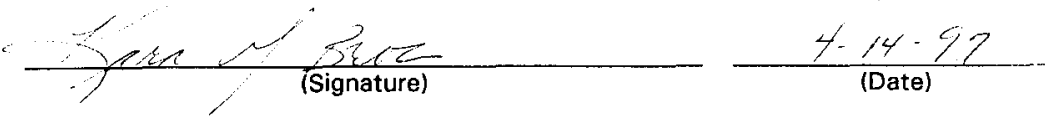

* Required only for full revisions transmitted to OSTI.

* * Required only for full revisions. 


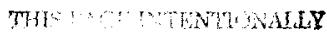
1. $\because \mathrm{N} N \mathrm{NK}$ 


\section{RELEASE AUTHORIZATION}

Document Number: HNF-SP-1104, Rev. 4

Document Title: $\quad$ Spent Nuclear Fuel Project Multi-Year Work Plan WBS \#1.4.1

Release Date: $\quad 4 / 14 / 97$

This document was reviewed following the procedures described in WHC-CM-3-4 and is:

APPROVED FOR PUBLIC RELEASE

WHC Information Release Administration Specialist:

-




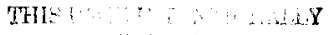

(... . N 


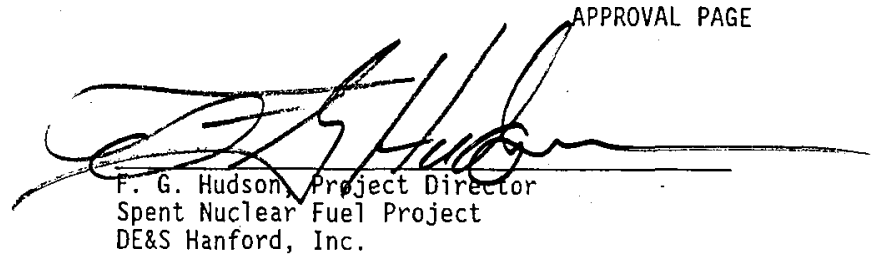

MQtide elen

N. H. Williams, Project Director Spent Nuclear Fuel Project Fluor Danjel Hanford, Inc.

\section{$\frac{4-1-97}{\text { Date }}$}

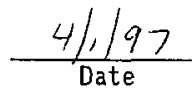

E. D. Sellers, Director

Spent Nuclear Fuels Project Division

U.S. Department of Energy

Richland Operations Office

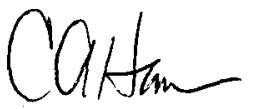

C. A. Hansen, Assistant Manager

for Waste Management

U.S. Department of Energy

Richland Operations Office

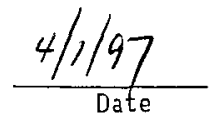




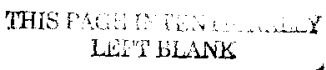


SPENT NUCLEAR FUEL PROJECT

WBS 1.4 .1

HNF-SP-1104. Rev 4

\section{Section}

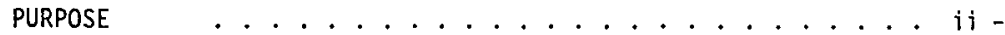

1.0 TECHNICAL BASELINE

1.0 Technical Baseline Narrative ............. . . . . 1.0-1

Exhibit 1A Hanford Site Technical Baseline Data . . . . . . . 1.A-1

2.0 WORK BREAKDOWN STRUCTURE

2.1 WBS Hierarchy . . . . . . . . . . . . . . . 2.1-1

2.2 WBS Dictionary . . . . . . . . . . . . . . . 2.2-1

2.3 WBS Responsibility Assignment Matrix . . . . . . . . . 2.3-1

3.0 SCHEDULE BASELINE

3.1 Program Master Basel ine Schedule . . . . . . . . . 3.1-1

3.2 SNF Level 1 Schedule . . . . . . . . . . . . . . . . 3.2-1

3.3 SNF Level 2 Schedule ................. . . . . . . .

3.4 Milestone/Performance Agreement List . . . . . . . . . . 3.4-1

3.5 Milestone Description Sheets . . . . . . . . . 3.5-1

\subsection{COST BASELINE}

4.1 B/A and Cost Baseline Summaries by Year . . . . . . . . 4.1-1

4.2 Basis of Estimate ................ . . . . . . . . . .

4.3 Planned Staff Profiles (AVE FTEs) . . . . . . . . . . 4.3-1

5.0 ASSUMPTIONS

5.1 Assumptions

5.1.1 Technical...................5.1.1-1

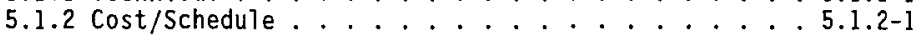

5.2 Risk

5.2.1 Technical .................. 5.2.1-1

5.2 .2 Cost/Schedule ............. . . 5.2.2-1 
THIS MAT RTENTENLA 1. $1 \mathrm{NWK}$ 


\section{SPENT NUCLEAR FUEL PROJECT \\ WBS 1.4.1}

HNF-SP-1 104, Rev 4

\section{PURPOSE}

The Spent Nuclear Fuel (SNF) Project Multi-Year Work Plan (MYWP) is a controlled living document that contains the current SNF Project Technical, Schedule and Cost Baselines. These baselines reflect the current Project execution strategies and are controlled via the change control process. Other changes to the MYWP document will be controlled using the document control process. These changes will be processed as they are approved to keep the MYWP a living document. The MYWP will be maintained continuously as the project baseline through the life of the project and not revised anually. The MYWP is the one document which summarizes and links these three baselines in one place. Supporting documentation for each baseline referred to herein may be impacted by changes to the MYWP, and must also be revised through change control to maintain consistency. 


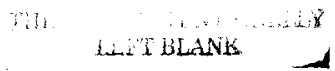




\section{SPENT NUCLEAR FUEL PROJECT \\ WBS 1.4.1}

HNF-SP-1104, Rev 4

\subsection{PROJECT TECHNICAL BASELINE}

The Hanford Site Technical Baseline (HSTB) was developed to consolidate the technical baselines for all projects across the Hanford Site and reflects the integration of the site-level and project-level baselines. The Spent Nuclear Fuel (SNF) Project technical baseline, as identified in the HSTB, is reproduced in Exhibit $1 A$, and is contained in Section 1 of this document.

At the Site-level, the data reflects guidance from the Hanford Mission Direction Document (MDD) ${ }^{\uparrow}$, which identifies safety objectives, interim and final endpoint targets, and performance measures. It is organized by material category and geographic area. The material category of interest to the SNF Project is spent nuclear fuel. The geographic areas of interest to the Project are Reactors on the River, the Central Plateau, and the South 600 Area. Selected interfaces with other Hanford Site projects are also included in Exhibit 1A. Section 3.1 contains the Program Master Baseline Schedule which reflects these site-level interfaces.

From the Project-level, the details of the SNF Project technical baseline are contained in the SNF Project Technical Baseline Description ${ }^{2}$. This document and the supporting information identify the Project-level functions and requirements necessary to accomplish the Project mission. The HSTB data in Exhibit $1 \mathrm{~A}$ reflects the SNF Project baseline data as well as site-wide baseline data that applies to the SNF Project.

Like the HSTB, the SNF Project technical baseline is composed of the constantly changing technical baselines of its sub-projects. As described in the SNF Project Systems Engineering Management Plan (WHC-SD-SNF-SEMP-001), the technical baseline evolves under tight configuration control from a functional requirements baseline (characterized by a Functional Design Criteria) to an operational baseline (characterized by authorization bases documents). Figure 1-l depicts the relationship of various technical baselines along with key documents which may contribute to each and how they progressively feed the Project and Site Integrated (technical, cost, and schedule) baselines. A complete iisting of document HNF-SD-SNF-PMP-011 is in the Project Management Plan (PMP).

The HSTB database is undergoing a significant revision to reflect the numerous changes that occurred as a result of the transition to the Project Hanford Management contractor (PHMC). The PHMC has committed to integrating this revision across the Site and projects by July 1997.

1 DOE/RL-96-14, Draft Hanford Mission Direction Document, U.S. Department of Energy, Richland Operations Office, June 1996.

2 Spent Nuclear Fuel Project Technical Baseline Description, WHC-SDSNF-SD-005, Revision 1, August 1996. 


\section{SPENT NUCLEAR FUEL PROJECT \\ WBS 1.4.1}

HNF-SP-1104, Rev 4

The purpose of this technical narrative section is to provide a summary of the SNF Project technical base]ine and background information on the data contained in Exhibit $1 \mathrm{~A}$.

\subsection{Project Summary}

The SNF Project was formed in early 1994 to specifically address the urgent need to move metallic uranium fuel from the present degraded storage conditions in the $K$ Basins near the Columbia River to safe interim storage on the Centra] Plateau until final disposition is decided at the national level.

1.1.1 Project Background. About $80 \%$ of the U.S Department of Energy's (DOE) spent nuclear fuel inventory is located at the Hanford $K$ Basins. Although the basins originally served the K Reactors in the 1950s and 1960s, N Reactor fuel was accumulated from 1978 through 1987. Storage at K Basins was intended to be only as needed to sustain operation of $\mathrm{N}$ Reactor while the PUREX Plant was refurbished and restarted. Although much of the $\mathrm{N}$ Reactor fuel was processed as planned, the decision in December 1992 to deactivate the PUREX Plant left approximately 2,100 metric tons of $N$ Reactor spent fuel in the $K$ Basins with no means for near-term removal and processing. Fuel stored in the basins exists in a degraded state which continues to further corrode. While fuel in the K-West Basin is contained in lidded canisters, the fuel in K-East Basin remains in open canisters allowing release of fission products to the basin water.

Deficient conditions at the $K$ Basins were identified in a number of internal and external reviews, including November 1993 findings of the DoE Spent Fuel Working Group ${ }^{3}$. Their report listed $K$ Basins among the few DOE spent nuclear fuel facilities given the highest priority to resolve environmental, health, and safety vulnerabilities.

In May 1994, the Defense Nuclear Facility Safety Board (DNFSB) issued Recommendation $94-1^{4}$ which identified concerns related to the U.S. DOE'S legacy fissile materials remaining from past defense production activities, including spent nuclear fuel stored at the $K$ Basins. The DNFSB expressed concern about the existing storage conditions for these materials and the slow pace at which the conditions were being remediated. The DNFSB made a strong recommendation to accelerate placement of the deteriorating fuel into a stable configuration that would minimize further degradation untio a final disposition option is selected.

DOE Spent Nuclear Fuel Working Group Report on Inventory and Storage of the Department's Spent Nuclear Fuel and Other Reactor Irradiated Nuclear Materials and the Environmental, Safety, and Health Vulnerabilities, U.S. Department of Energy, November 1993.

4 Conway, J.T., DNFSB Recommendation 94-1, letter to. H.R. O'Leary, U.S. Department of Energy, May 26, 1994. 


\section{SPENT NUCLEAR FUEL PROJECT WBS 1.4.1}

HNF-SP-1104, Rev 4

Additionally, negotiations with signatories of the Hanford Federal Facility Agreement and Consent Order (Tri-Party Agreement) defined a target milestone to achieve fuel removal by 2002 .

In addition to the fuel stored at the $K$ Basins, other spent nuclear fuel stored in the 400 Area Interim Storage Area (ISA); Fast Flux Test Facility (FFTF), T Plant; 324, 325, and 327 Buildings; and the $\mathrm{N}$ Reactor Basin will be moved to safe interim storage on the Central Plateau or transported offsite for treatment, consistent with national plans for DOE-owned spent nuclear fue?. 


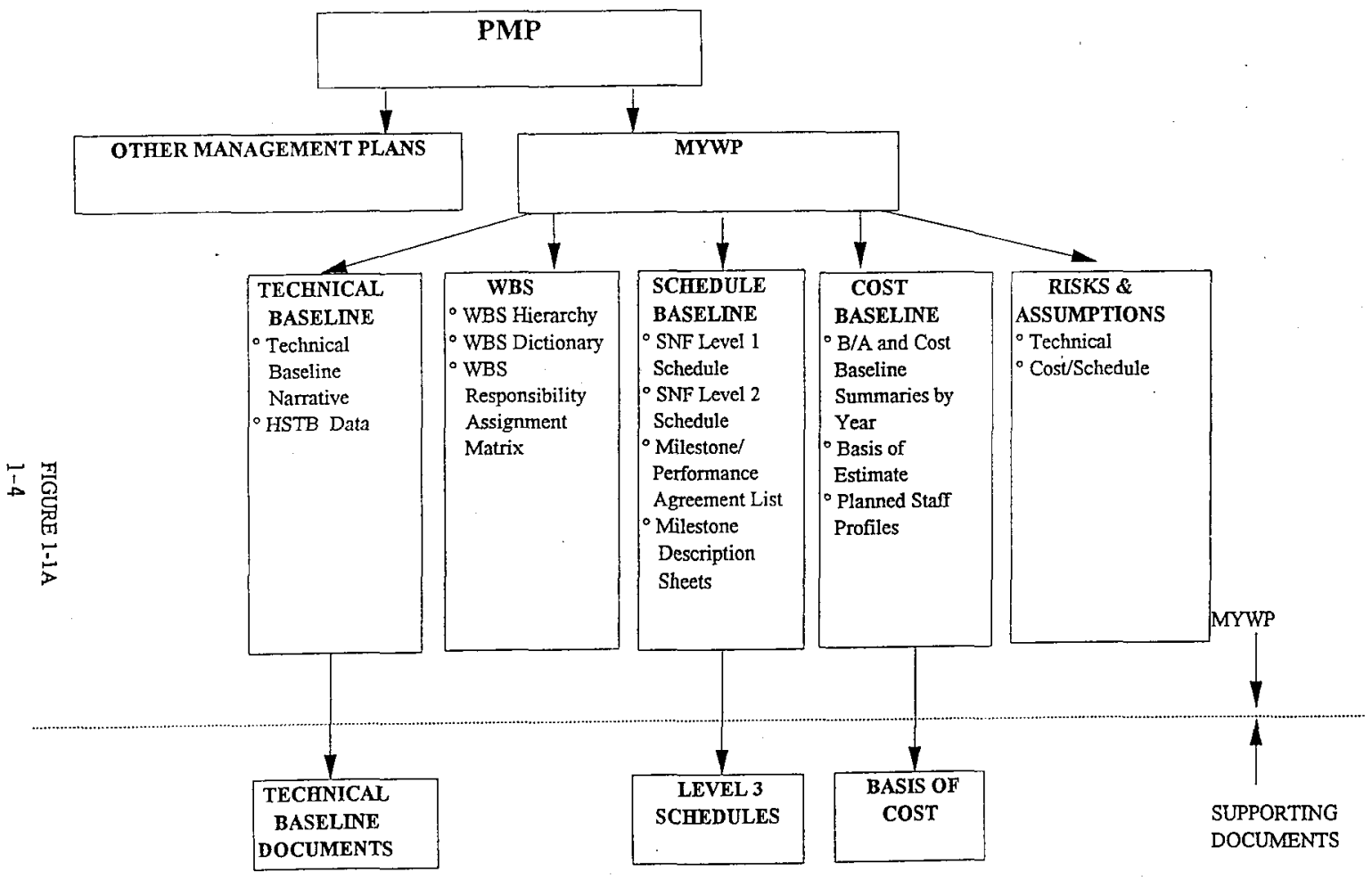




\section{TECHNICAL BASELINE DOCUMENT SUMMARY}

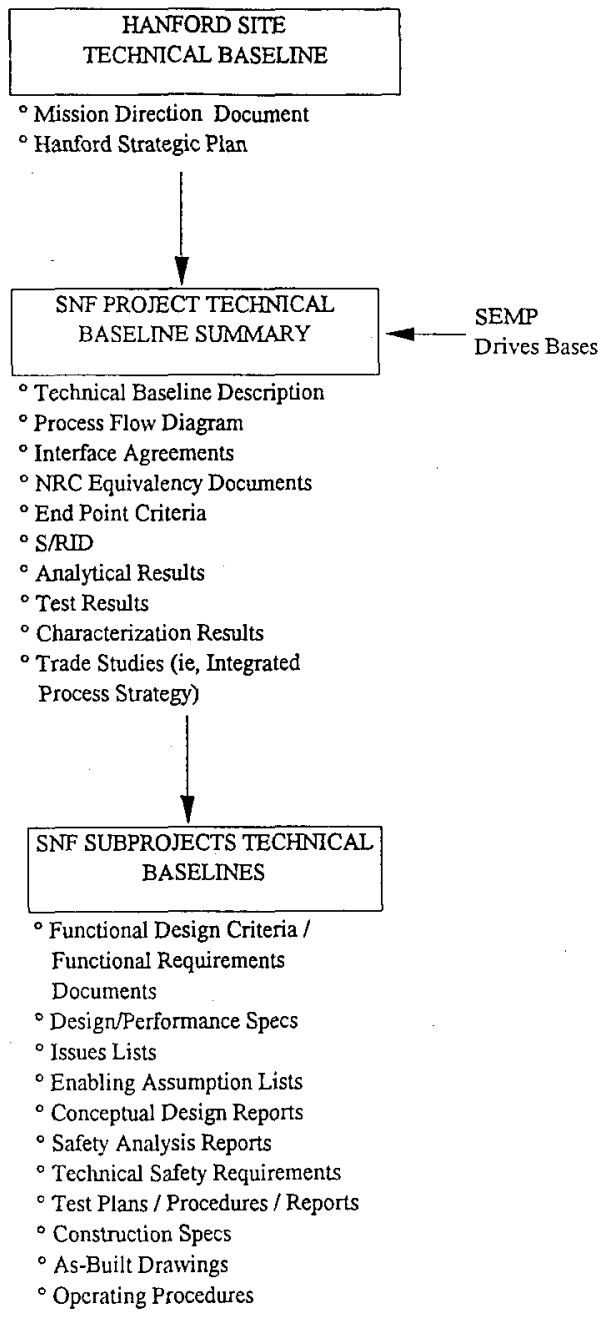




\section{SPENT NUCLEAR FUEL PROJECT \\ WBS 1.4.1}

HNF-SP-1104, Rev 4

1.1.2 Technical Summary.

The SNF Project process was developed to remove the fuel from existing canisters, repackage in MCOs for cold vacuum drying to remove bulk water from the fuel. MCOS will be transferred to the CSB for staging prior to hot conditioning to enable interim storage in the CSB.

$K$ Basin Operations and Maintenance (WBS 1.4.1.13.01) Ongoing operations and maintenance assures safe storage of the $K$ Basins SNF until fuel, sludge, and debris removal are complete. $K$ Basins Operations and Maintenance provides for all activities and facilities required to directly support the minimum safe operations of the basins including material and facility surveillance, radiological control, material and waste handling, safeguards and security, maintenance, operation of utility systems, planning/scheduling, engineering, training and assessments. The basin facilities will become the responsibility of Transition Projects at the end of the SNF Project in FY 2001.

Fuel Retrieval (WBS 1.4.1.14.02) and FRS Operations (WBS 1.4.1.13.02) The fuel retrieval process is located within each Basin pool where fuel elements will be removed from existing canisters and cleaned to remove unbonded sludge and corrosion particles. Fuel elements and fuel scraps will loaded into separate tier baskets. Loaded tier baskets are transferred to a queue station for lag storage prior to placement in the MCO.

Multi-Canister Overpack (WBS 1.4.1.15.01)

The MCO will provide primary confinement for fue] elements during transport, conditioning, and storage. Each MCO can accommodate a total of five Mark IV fuel baskets or six Mark $1 A$ fuel baskets. The current baseline assumes that MCOs will contain no more than one basket loaded with fuel scraps. The MCO 7 id main seal is mechanically sealed at the $K$ Basins loadout pit prior to transport to Cold Vacuum Drying facility. Pressure relief valves and rupture disks provide overpressure protection.

Cask/Transportation (WBS 1.4.1.15.02)

The cask/transportation system will be utilized to load tier baskets into MCOs and transfer the loaded MCO/cask to the CVD facility and to the CSB. The current baseline assumes that five cask/transporter systems will be sufficient to maintain the processing schedule.

Cold Vacuum Drying (WBS 1.4.1.17.01) and CVD Operations (WBS 1.4.1.13.04) The CVD facility is located in the $100 \mathrm{~K}$ Area to serve both the $\mathrm{K}$ East and $K$ West Basins. There, free and some hydrated water will be removed from the MCO under vacuum at sightly elevated temperatures. The project will then transport the water back to the K-Basins for return to the basins via the Integrated Water System. Four processing stations are provided in the CVD. Following the drying process, the MCOS will be sealed and transported to the CSB in the 200 Area for staging. 


\section{SPENT NUCLEAR FUEL PROJECT \\ WBS 1.4.1}

HNF-SP-1104, Rev 4

Canister Storage Building (WBS 1.4.1.16.01) and CSB Operations (WBS

1.4.1.13.08)

The CSB provides staging capacity for MCOs prior to hot conditioning. On receipt at the CSB service pit, the cask lid will be removed, the MCO vented and purged with inert gas, and then resealed for staging. Throughout the staging period, pressure relief devices provide $M C O$ overpressure protection. The CSB contains three storage vaults and an annex for the Hot Conditioning System. Vault 1 is outfitted with 220 carbon steel storage tubes which can each hold two MCOs. A storage tube plug provides shielding and secondary confinement for the MCO and maintains the inert atmosphere within the storage tube. Vault 1 is sized to accommodate all the MCOs needed for $K$-Basins SNF. The MCO Handling Machine (MHM) is used to transfer MCOs within the CSB and HCS. Following hot conditioning, a cap with rupture disk will be seal-welded on the $\mathrm{MCO}$, which will be returned to a CSB storage tube for interim storage until final disposition plans are decided at the national level. The CSB is designed for an interim storage period of 40 years, extendable to 75 years with future refits or upgrades. For planning purposes, it is assumed that CSB Operations become the responsibility of Solid Waste Programs at the end of the SNF Project in FY 2001.

Hot Conditioning System (WBS 1.4.1.17.02) and HCS Operations (WBS 1.4.1.13.07) Six process stations are contained in the Hot Conditioning Annex to the CSB. Hot conditioning will remove hydrated water remaining in the fuel and reduce fuel reactivity. The hot conditioning step, performed by holding the MCO at $300^{\circ} \mathrm{C}$ under vacuum followed by a partial oxidation step using helium with a maximum $2 \%$ oxygen content, is needed to prevent excessive pressure buildup that could be caused by continuing corrosion during interim storage.

Sludge Removal (WBS 1.4.1.14.03) and Sludge Removal Operations (WBS $1.4 .1 .13 .06)$

In support of the cleanout of the $K$ Basins, Sludge Removal systems (SRS) are provided to manage the siudge/sediment on the floor and in the pits of the basins and the sludge/sediment in the fuel canisters. The current baseline assumes that $K$ East Basin canister and floor sludge will be accumulated in the basin Weasel Pit for transfer to the Tank Waste Remediation System (TWRS) for management with other tank wastes. The SRS assumes (1) the $K$ West Basin canister sludge will be collected by the Integrated Water System into a retrievable form and (2) the sludge has constituents that make it consistent with $K$ East Basin canister sludge and it will be dispositioned in a similar manner. Because of the relatively small volume, $K$ West Basin floor sludge will remain in the basin.

Debris Removat (WBS 1.4.1.14.04) and Debris Removal Operations (WBS 1.4.1.13.03)

The Debris Removal system is provided to move, clean, and remove empty fuel canisters and other debris within the basins to facilitate fuel and sludge removal. Debris will be disposed of through the Hanford Solid Waste Program. 
Integrated Water System (WBS 1.4.1.14.05) and Water Treatment Operations (WBS $1.4 .1 .13 .05)$

The Integrated Water Treatment System (IWTS) provides water filtration and treatment necessary to maintain water quality in the basins. It will upgrade and provide new water treatment systems and facilities for both the KE and KW basins. The IWTS will provide clean, treated, or fresh water and receive and treat contaminated water for the fuel, sludge, and debris removal systems, as well as the MCO/Cask system. The IWTS will provide treatment capability to maintain water clarity, remove decay heat, and maintain soluble and insoluble radionuclide concentrations in the basins as low as reasonably achievable during continued SNF storage and fuel and sludge removal activities. The IWTS will also install a system for canister sludge management during fuel remova?. This system will put filter backwash material into the Weasel Pit.

Facility Uparades (WBS 1.4.1.14.01)

Facility upgrades are performed to repair, replace, and modify essential $K$ Basins infrastructure to maintain safe operations and to facilitate fuel removal from the Basins, including dose reduction and modifications to support the cask/transportation system.

0ther Hanford Fuel (WBS 1.4.1.15.03)

Fuel stored in the 400 Area ISA will be moved to the 200 Area for storage or transferred offsite for treatment. Sodium bonded Fast Flux Test Facility (FFTF) fuel will be loaded into shipping casks in the CSB and shipped to Argonne National Laboratory-West for treatment. FFTF fuel that requires additional security measures will be stored in the Plutonium Finishing Plant. Remaining FFTF fuel and other fuel in interim storage casks in the 400 Area ISA will be transferred to the 200 Area ISA adjacent to the CSB. Shippingport PWR Core 2 assemblies stored at T Plant will be retrieved and placed in MCOs for storage inside the CSB.

The SNF Project also has the responsibility for planning for disposition of miscellaneous reactor fuel assemblies located at the PFP and TRIGA Reactor fuel located in the 200 West Area burial ground. If fuel is discovered during cleanout of the $N$ Reactor basin, the SNF Project has the responsibility for its packaging, transport, and storage.

1.1.3 Interface with Hanford Site Mission. The SNF Project mission supports the overall Hanford Site mission by directly supporting Hanford's Mission Execution Priorities as identified in the MDD. The MDD priorities are:

- Essential Safety Operations -- These are operations that must be conducted to protect the health and safety of the public, workers, and environment. Maintaining safe storage of irradiated fue 1 in the $\mathrm{K}$ Basins is identified as an essential safety objective for the Site.

- Mitigate Urgent Risks -- DOE-EM has identified three urgent risks across the complex, all of which are present at Hanford: unstable plutonium, highlevel waste tanks, and corroded spent nuclear fuel. Included among essential actions listed in the MDD supporting this priority is moving spent fuel away from the Columbia River. 


\section{SPENT NUCLEAR FUEL PROJECT WBS 1.4.1}

HNF-SP-1104, Rev 4

- Reduce/Eliminate Costly Mortgages -- This priority addresses deactivation of facilities that require substantial resources for surveillance and maintenance. Movement of other Hanford SNF from current storage locations, such as the FFTF, the $N$ Reactor Basin, and the 300 Area laboratory buildings enables those facilities to be deactivated.

- Stabilize/Contain Wastes -- This priority addresses actions required for waste management that are not addressed in the other priorities.

- Recover Land (and Other Resources) for Beneficial Use -- This priority addresses achievement of land use goals for the major geographic areas of the site. The SNF Project directiy supports achievement of the draft land use goal for the Reactors on the River geographic area to "remove or stabilize spent fuel, surplus facilities, and waste sites to protect groundwater and the Columbia River".

\subsection{Project End Point Targets}

The Project technical objectives include the endpoint targets identified in the MDD for the material category "Spent Fuel" that are applicable to WBS element 1.4.1. The endpoint targets identified in Table 1-1 in Exhibit 1A reflect the stated MDD target of completion of spent fuel removal by $12 / 31 / 99$ and transfer of the $K$ Basins to Decontamination \& Decommissioning (D\&D). Since the MDD was prepared, the fuel removal schedule has changed and DOE has determined that the $K$ Basins will be transferred to Transition Projects after completion of the SNF Project mission. Table 1.2-1 below, summarizes the endpoint targets contained in Exhibit $1 \mathrm{~A}$ and reflects current SNF Project/Transition Projects plans.

\section{Table 1.2-1 Endpoint targets for Material Category "Spent Fuel"}

\begin{tabular}{|c|c|c|}
\hline Reactors on the River & Central Plateau & South 600 Area \\
\hline $\begin{array}{l}\text { Spent fuel removed by } 7 / 31 / 00 \\
\text { K-Basins cleaned sufficient to } \\
\text { transition to Transition Projects by } \\
9 / 30 / 01\end{array}$ & $\begin{array}{l}\text { Spent fuels consolidated in the } 200 \\
\text { Area in safe, stable, cost-effective } \\
\text { interim storage pending national } \\
\text { decisions on their ultimate } \\
\text { disposition. }\end{array}$ & $\begin{array}{l}\text { Spent fuels (light water reactor) } \\
\text { removed to interim storage in } 400 \\
\text { Area pending cvalability of } 200 \\
\text { Area interim storage. } \\
\text { Spent fuels (TRIGA and light } \\
\text { water reactor) and applicable } \\
\text { FFTF fuels removed from } 400 \\
\text { interim storage area to } 200 \text { Area. } \\
\text { Spent fuels (sodium-bonded } \\
\text { FFTF) removed off-site for final } \\
\text { disposition }\end{array}$ \\
\hline
\end{tabular}




\subsection{Summary-Level Forecasting Data}

1.3.1 Waste Type Data. Section 1.3.1 in Exhibit 1A contains the waste (Solid Waste, Tank Waste, Liquid Effluents, and Special Case Waste) inventory and volume projection data. These data are used to track the waste through generation, transfer, receipt, storage, and disposition. The Project schedule and budget reflect the $p l a n s$ for disposition of waste until the turnover of the $\mathrm{K}$ Basins to the Transition Projects and the turnover of the Canister Storage Building to the successor operating organization, assumed to be the Solid Waste Program for $\mathrm{planning} \mathrm{purposes.}$

The solid waste data were provided from the Solid Waste Information Tracking System (SWITS) database. This database is maintained by the Solid Waste program and receives input from a 71 Hanford Site and offsite waste generators, including the SNF Project. Exhibit $1 A$ identifies projected liquid waste from two sources: (1) water drained from multi-canister overpacks (MCO) during Cold Vacuum Drying (CVD) processing of spent nuclear fuel and (2) water shipped from the $K$ Basins during fuel removal to maintain the required basin levels. Liquid waste volumes may also be affected by tritium-level reduction efforts in the $\mathrm{K}$ East basin. Plans to return the water drained from the MCOs in the CVD to the K Basins as well as changes in the time-phasing of waste generation resulting from changes in the SNF processing schedule will be reflected in the July 1997 update of Exhibit 1A.

No data were forecast beyond FY 2001 as planning for activities beyond achieving interim storage is outside the current SNF Project scope. However, Exhibit 1A shows that the SNF Project maintains responsibility for disposition of more than 6,000 cubic meters of low-level liquid waste from FY 2001 through FY 2004. An RL Memorandum of Understanding with the Spent Nuclear Fuel Project, Transition Projects, a nd Restoration Projects was issued in October 1996 assigning responsibility for disposition of the basin water to the Transition Projects. This change will be reflected in the Juiy 1997 revision of Exhibit $1 A$.

1.3.2 Nuclear Materials. Section 1.3.2 of Exhibit 1A contains the nuclear materials (Nuclear Fue1) inventory and volume projection data. This data is used to track the nuclear materials through transfer, receipt, storage, and disposition. The Project schedule and budget reflect the plans for disposition of nuclear materials. Nuclear materials data from the SNF Project is derived from three sources:

- Irradiated fuel stored in the $\mathrm{K}$ Basins and scheduled to be moved to the Canister Storage Building. It will be transferred to a successor

organization at the conclusion of the SNF Project in FY 2001. For planning purposes, this is assumed to be the Solid Waste Program.

- Irradiated fuel stored in Hanford Site locations other than the $K$ Basins. This fuel will be moved to SNF Project operational control within the 200 Area from current storage locations or from the 400 Area Interim Storage Area. It will be transferred to a successor organization at the conclusion 


\title{
SPENT NUCLEAR FUEL PROJECT WBS 1.4.1
}

HNF-SP-1104, Rev 4

of the SNF Project in FY 2001 or transferred to the Idaho National

Engineering Laboratory for treatment and final disposition (sodium-bonded FFTF fuel on $7 y$ ). For planning purposes, the successor organization is assumed to be the Solid Waste Program.

- Sludge (corroded cladding, oxidized fuel, fuel particles, and inorganic and organic debris) within the K. Basins which will be assumed to be spent nuclear fuel until time of transfer for disposal; at that time it will be transferred to double-shell tanks or other systems for disposition as waste. The sludge inventory is not reflected in the HSTB database.

No data were forecast beyond FY 2001 because planning for activities beyond achieving interim storage is outside the current SNF Project scope.

1.3.3 Facilities (excess, deactivated). Section 1.3.3 in Exhibit 1A contains a listing of primary structures/facilities within the SNF Project. An MOU between DOE-SFD and DOE-WPD in August 1996 transferred the management responsibility for the $1706 \mathrm{KE} / \mathrm{KER} / \mathrm{KEL}$ facility to the SNF Project. The 7 ist does not reflect future facilities, including Canister Storage Building, and Hot Conditioning System Annex or non-permanent structures such as mobile offices. Future facilities will be included in the facilities listing after completion of construction. SNF Project mobile offices in the $100 \mathrm{~K}$ Area are noted below, and are planned for disposition in a FDH inter-program agreement completed in December 1996.

\begin{tabular}{|c|c|}
\hline 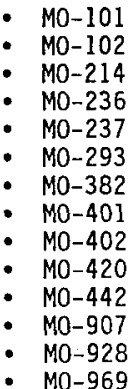 & $\begin{array}{l}1711 \mathrm{~K} \\
1709 \mathrm{~K} \\
1701 \mathrm{KA} \\
1728 \mathrm{~K} \\
1729 \mathrm{~K} \\
1725 \mathrm{~K} \\
1721 \mathrm{~K} \\
1719 \mathrm{~K} \\
1718 \mathrm{~K} \\
--2-- \\
1726 \mathrm{~K} \\
1722 \mathrm{~K} \\
1723 \mathrm{~K} \\
1730 \mathrm{~K}\end{array}$ \\
\hline
\end{tabular}

\author{
Document Control \\ Ops Analysis \& Waste Handiing \\ Job Control (ex Patrol Badge House) \\ Ops/Hpt Change Trailer (105KW) \\ Construction Forces trailer (105KW) \\ Projects and Training/Procedures \\ Radiological Control \\ $K$ Basins Ops/Admin and DOE \\ $K$ Basins Engineering \\ Sample Trailer next to 183KE \\ Training Classrooms \\ Ops Analysis \& Waste Handling \\ Design/Drafting \\ Ops/Hpt Change Trailer (105KE)
}

1.3.4 Infrastructure. Section 1.3.4 in Exhibit IA contains data for Hanford Site Infrastructure planning purposes only. Due to differences in definition of infrastructure type and the financial structure, a direct correlation can not be made between the forecasted usage and budgets within this MYWP.

\subsection{Drivers}

A number of documents provide the specific strategic, technical, and regulatory direction that govern how the Project mission is executed. These documents serve as the primary source of requirements for the Project. 


\section{SPENT NUCLEAR FUEL PROJECT \\ WBS 1.4.1}

HNF-SP-1104, Rev 4

Section 1.4.1 and 1.4.2 of Exhibit IA list the key Project drivers and other drivers.

1.4.1 Key project drivers. Key project drivers are those that drive the mission and direction of the Project. These are identified as sources of Mission Requirements in the SNF Project Technical Baseline Description. Key drivers include legal commitments and obligations, NEPA documents, DOE plans, guidance documents, and key directives, letters providing direction to the Project, and other documents that impact workscope or the method in which workscope is performed.

1.4 .2 other drivers. The SNF Project is subject to the full range of applicable local, State and Federal laws and regulations. The administrative and technical requirements contained in the laws and regulations impact the products produced by the SNF Project and subprojects. In addition, the administrative requirements (e.g., permitting) significantly impact the timing and sequencing of planned project activities.

The Code of Federal Regulations (CFR) is the source document for many of the implementing regulations associated with federal law. Permits are jssued by the regulatory agencies for specific activities when required by applicable laws and regulations. The permits contain requirements and constraints that directly impact the activities for which they are issued. In addition to the requirement to have a permit, the specific requirements of the permit will impact the project activities.

Washington state publishes legal requirements in the form of the Revised Code of Washington (RCW) and the Washington Administrative Code (WAC). These in turn may be passed on as direction by DOE-RL. The most significant of these are those associated with water and air permitting. Permits are required for anticipated air and liquid effluent emissions.

Standards/Requirements Identification (S/RIDs) is the process of identifying the specific Environmental, Safety, and Health standards/requirements that apply to conducting the day-to-day mission of defense nuclear facilities. The standards/requirements are derived from the following 1 ist of potentially applicabie documents:

- DOE Orders, Secretary of Energy Notices, Rules, and directives;

- Federal Regulations and Laws;

- National Consensus issuances and industry codes and standards: National Fire Protection Association, American National Standards

Institute/American Nuclear Society for Quality Control, Institute of Electronics and Electrical Engineers, International Commission on Radiological Protection, Underwriters Laboratory, Institute of Nuclear Power Operations, Electric Power Research Institute, etc.;

- International guidance: International Atomic Energy Agency, etc.

- other State and local requirements.

The product of the S/RIDs process, a facility-specific S/RID document is approved by $\mathrm{RL}$. Additional requirements are derived from DOE plans, guidance 


\section{SPENT NUCLEAR FUEL PROJECT WBS 1.4.1}

HIN-SP-1104, Rev 4

documents and directives, letters providing direction to the Project, company manuals, and other technical and administrative documents. 


\section{SPENT NUCLEAR FUEL PROJECT WBS 1.4.1}

HNF-SP-1104, Rev 4

\subsection{Functional Definitions}

In 1994, the Hanford Site Systems Engineering activity developed five major functions that encompass all activities in support of the Site's primary function, "Clean Up Hanford". The SNF Project and other Hanford Site projects, such as the Tank Waste Remediation System and Transition Projects, are responsible for performance of these functions. The SNF Project Systems Engineering activity established its function structure from the Site's by further developing the five functions listed below.

- Function 1.0, Manage SNF Project

- Function 2.0, Acquire Mission Essential Capabilities

- Function 3.0, Obtain Public Involvement

- Function 4.1.1.8, Deactivate K Basin

- Function 4.7.2, Condition, Store, and Disposition SNF Material.

The Site and SNF Project functional structure are shown in Figure 1-2. The HSTB database includes SNF Project functions one level below the intersection with the Site functions identified above. The SNF Project Technical Baseline Description includes those functions as well as any lower-level SNF Project functions that were developed in support of subproject activities.

Table 1.5-1 shows the relationship between the SNF Project systems engineering functions and the SNF Project Work Breakdown Structure. Section 1.5 of Exhibit $1 \mathrm{~A}$ identifies and defines the SNF Project functions contained in the HSTB database.

Table 1.5-1 SNF Project Function/Work Breakdown Structure Crosswalk

\begin{tabular}{|c|c|c|c|}
\hline Functiont & Function Title & WBS & uss litle \\
\hline 1.0 & Manage SNF Project & $\begin{array}{l}1.4 .1 .00 \\
1.4 .1 .12 \\
\end{array}$ & $\begin{array}{l}\text { Project Direction } \\
\text { Project Integration }\end{array}$ \\
\hline 1.1 & Integrate SNF Project & $\begin{array}{l}1.4 .1 .00 \\
1.4 .1 .12 \\
\end{array}$ & $\begin{array}{l}\text { Project Direction } \\
\text { Project Integration }\end{array}$ \\
\hline 1.2 & $\begin{array}{l}\text { Manage SNF Project Technical } \\
\text { Baseline }\end{array}$ & 1.4 .1 .12 & Project Integration \\
\hline 1.3 & Provide Support Services & 1.4 .1 .12 & Project Integration \\
\hline 1.4 & $\begin{array}{l}\text { Manage SNF Project Regulatory } \\
\text { Compl iance }\end{array}$ & 1.4 .1 .11 .00 .02 & Regulatory Compliance \\
\hline 2.0 & $\begin{array}{l}\text { Acquire SNF Mission Essential } \\
\text { Capabilities }\end{array}$ & $\begin{array}{l}1.4 \cdot 1.14 .01 \\
1.4 .1 .14 .02 \\
1.4 .1 .14 .03 \\
1.4 .1 .14 .04 \\
1.4 .1 .14 .05 \\
1.4 .1 .15 .01 \\
1.4 .1 .15 .02 \\
1.4 .1 .15 .03 \\
1.4 .1 .16 .01 \\
1.4 .1 .17 .01 \\
1.4 .1 .17 .02 \\
\end{array}$ & $\begin{array}{l}\text { Facility Upgrades } \\
\text { Fuel Retrieval Systen } \\
\text { Sludge Removal } \\
\text { Debris Removal } \\
\text { Integrated Water System } \\
\text { MCO } \\
\text { Cask/Transportation } \\
\text { Other Hanford SNF } \\
\text { CSB } \\
\text { Cold Vacuum Drying } \\
\text { Hot Conditioning System }\end{array}$ \\
\hline 2.1 & Formulate Acquisition Strategy & \multicolumn{2}{|c|}{ Work completed prior to FY 1997} \\
\hline 2.2 & Provide Expertise & 1.4 .1 .12 & Project Integration \\
\hline
\end{tabular}




\section{SPENT NUCLEAR FUEL PROJECT WBS 1.4.1}

HNF-SP-1104, Rev 4

Table 1.5-1 SNF Project Function/Work Breakdown Structure Crosswalk (eontinued)

\begin{tabular}{|c|c|c|c|}
\hline function \# & Eunction $1 \mathrm{itl}$ & ws * & WBS Title \\
\hline 2.3 & Provide Technology & \multicolumn{2}{|c|}{ Work completed prior to FY 1997} \\
\hline 2.4 & $\begin{array}{l}\text { Provide Facilities, Equipment, } \\
\text { Infrastructure \& Supplies }\end{array}$ & $\begin{array}{l}1.4 \cdot 1.14 .01 \\
1.4 .1 .14 .02 \\
1.4 .1 .14 .03 \\
1.4 .1 .14 .04 \\
1.4 .1 .14 .05 \\
1.4 .1 .15 .01 \\
1.4 .1 .15 .02 \\
1.4 \cdot 1.15 .03 \\
1.4 \cdot 1.16 .01 \\
1.4 .1 .17 .01 \\
1.4 .1 .17 .02 \\
\end{array}$ & $\begin{array}{l}\text { Facil ity Upgrades } \\
\text { Fuel Retrieval System } \\
\text { Sludge Removal } \\
\text { Debris Removal } \\
\text { Integrated Water system } \\
\text { MCO } \\
\text { Cask/Transportation } \\
\text { Other Hanford SHF } \\
\text { CSB } \\
\text { Cold Vacum Drying } \\
\text { Hot Conditioning System }\end{array}$ \\
\hline 2.5 & Provide Essential Information & $\begin{array}{l}1.4 \cdot 1.12 \\
1.4 .1 .11 \\
\end{array}$ & $\begin{array}{l}\text { Project Integration } \\
\text { Safety \& Quality }\end{array}$ \\
\hline 2.6 & $\begin{array}{l}\text { Provide Integrated independent } \\
\text { Service }\end{array}$ & 1.4 .1 .12 & Project Integration \\
\hline 3.0 & Obtain Publ ic involvement & $\begin{array}{l}1.4 .1 .00 \\
1.4 .1 .11 \\
\end{array}$ & $\begin{array}{l}\text { Project Direction } \\
\text { Safety \& Quality }\end{array}$ \\
\hline $\begin{array}{l}3.1 \\
3.2 \\
3.3 \\
3.4 \\
3.5\end{array}$ & $\begin{array}{l}\text { Identify Technical Baseline } \\
\text { Decisions } \\
\text { Perform Strategic Analysis \& } \\
\text { Develop Public Involvement Plan } \\
\text { Execute Public Involvement Process } \\
\text { Develop Understanding of } \\
\text { Public/Project Differences } \\
\text { Reconcile Differences }\end{array}$ & \multicolumn{2}{|c|}{ Work completed prior to Fy 1997} \\
\hline 4.1 .1 .8 & Deactivate $\mathrm{K}$ Basins & $\begin{array}{l}1.4 .1 .13 \\
1.4 .1 .14 \\
\end{array}$ & $\begin{array}{l}\text { Operations } \\
\text { Facility Projects }\end{array}$ \\
\hline 4.1 .1 .8 .1 & $\begin{array}{l}\text { Operate and Maintain } K \text { Basins } \\
\text { During Deactivation }\end{array}$ & 1.4 .1 .13 .01 & $\begin{array}{l}\text { K Basin Operations } 8 \\
\text { Maintenance }\end{array}$ \\
\hline 4.1 .1 .8 .2 & Plan $K$ Basins Deactivation & 1.4 .1 .13 .01 & $\begin{array}{l}\text { K Basin Operations \& } \\
\text { Maintenance }\end{array}$ \\
\hline 4.1 .1 .8 .3 & Disposition K Basins Materials & $\begin{array}{l}1.4 \cdot 1 \cdot 13 \\
1.4 \cdot 1.14 \\
\end{array}$ & $\begin{array}{l}\text { Operations } \\
\text { Facility Projects }\end{array}$ \\
\hline $\begin{array}{l}4.1 \cdot 1.8 .4 \\
4.1 .1 .9 \\
4.1 .1 .10 \\
4.1 .1 .11 \\
4.1 .1 .12 \\
4.1 .1 .13\end{array}$ & $\begin{array}{l}\text { Perform K Basins Deactivation } \\
\text { Deactivate Other SNF ( } 400 \text { Area) } \\
\text { Deactivate HCS } \\
\text { Deactivate CSB } \\
\text { Deactivate Other SNF ( } 200 \text { Area) } \\
\text { Deactivate CVD }\end{array}$ & \multicolumn{2}{|c|}{ Outside SNF Project scope } \\
\hline 4.7 .2 & $\begin{array}{l}\text { Condition, Store, \& Disposition SNF } \\
\text { Material }\end{array}$ & $\begin{array}{l}1.4 \cdot 1.13 .02 \\
1.4 \cdot 1.13 .03 \\
1.4 .1 .13 .04 \\
1.4 .1 .13 .06 \\
1.4 .1 .13 .07 \\
1.4 .1 .13 .08 \\
\end{array}$ & $\begin{array}{l}\text { FRS Operations } \\
\text { Debris Removal Operations } \\
\text { CVD Operations } \\
\text { Sludge Removal Operations } \\
\text { HCS Operations } \\
\text { CSB Operations }\end{array}$ \\
\hline 4.7 .2 .1 & Operate \& Maintain SNF Facilities & $\begin{array}{l}1.4 \cdot 1.11 \\
1.4 .1 .13 .01 \\
1.4 .1 .13 .04 \\
1.4 .1 .13 .07 \\
1.4 .1 .13 .08 \\
\end{array}$ & $\begin{array}{l}\text { Safety \& Quality } \\
\text { K Basins O \& } K \\
\text { CVD Operations } \\
\text { HCS Operations } \\
\text { CSB Operations }\end{array}$ \\
\hline
\end{tabular}




\section{SPENT NUCLEAR FUEL PROJECT \\ WBS 1.4.1}

HNF-SP-1104, Rev 4

Table 1.5-1 SNF Project Function/Work Breakdown Structure Crosswalk loontinuod

\begin{tabular}{|c|c|c|c|}
\hline functiont & Function title & HBS & was Iitle \\
\hline 4.7 .2 .2 & $\begin{array}{l}\text { Administer Condition, Store, \& } \\
\text { Disposition Operations }\end{array}$ & $\begin{array}{l}1.4 .1 .13 .01 \\
1.4 .1 .15 .03\end{array}$ & $\begin{array}{l}\text { K Basins } 0 \text { \& } M \\
\text { Other Hanford Fuel }\end{array}$ \\
\hline 4.7 .2 .3 & Stage K Basins SHF & 1.4.1.13.08 & CSB Operations \\
\hline 4.7 .2 .4 & Perform Hot Conditioning & 1.4 .1 .13 .07 & HCS Operations \\
\hline 4.7 .2 .5 & Store SNF & $\begin{array}{l}1.4 .1 .13 .08 \\
1.4 .1 .15 .03 \\
\end{array}$ & $\begin{array}{l}\text { CSB Operations } \\
\text { Other Hanford Fuel }\end{array}$ \\
\hline 4.7 .2 .6 & Stage SNF for Final Disposition & \multicolumn{2}{|c|}{ Outside SNF Project scope } \\
\hline
\end{tabular}

\subsection{Project Life-Cycle Requirements}

Requirements define how a function must be performed, describe conditions under which functions are performed, or set limits on functions or products. Requirements are derived from the source documents identified in section 1.4, Project Drivers. The requirements identified in Section 1.6 of Exhibit $1 \mathrm{~A}$ are those that have been allocated (determined to be applicable) to the functions at which the Project connects with the Site Baseline (the five functions listed in section 1.5).

\subsection{Project Issues and Assumptions}

A technical issue management system is used to identify, evaluate and resolve issues that encompass multiple subprojects and result in significant potential for baseline changes in the Project. In resolving these SNF Project 1evel issues, the decisions formally became part of the SNF Project's technical baseline.

SNF subprojects are required to maintain an issues management process to monitor resolution of issues at the subproject level. Subproject managers are responsible for regularly updating their issues 1ists. Likewise, subproject managers are responsible for maintaining and validating key enabling assumptions that affect their subproject and impact project design and safety bases.

A listing of all original issues and assumptions developed at the early stages of the SNF Project is retained in the electronic version of the SNF Project technical baseline database for historical records. See section 5.0 for current key Project assumptions. 


\section{SPENT NUCLEAR FUEL PROJECT WBS 1.4.1}

HNF-SP-1104, Rev 4

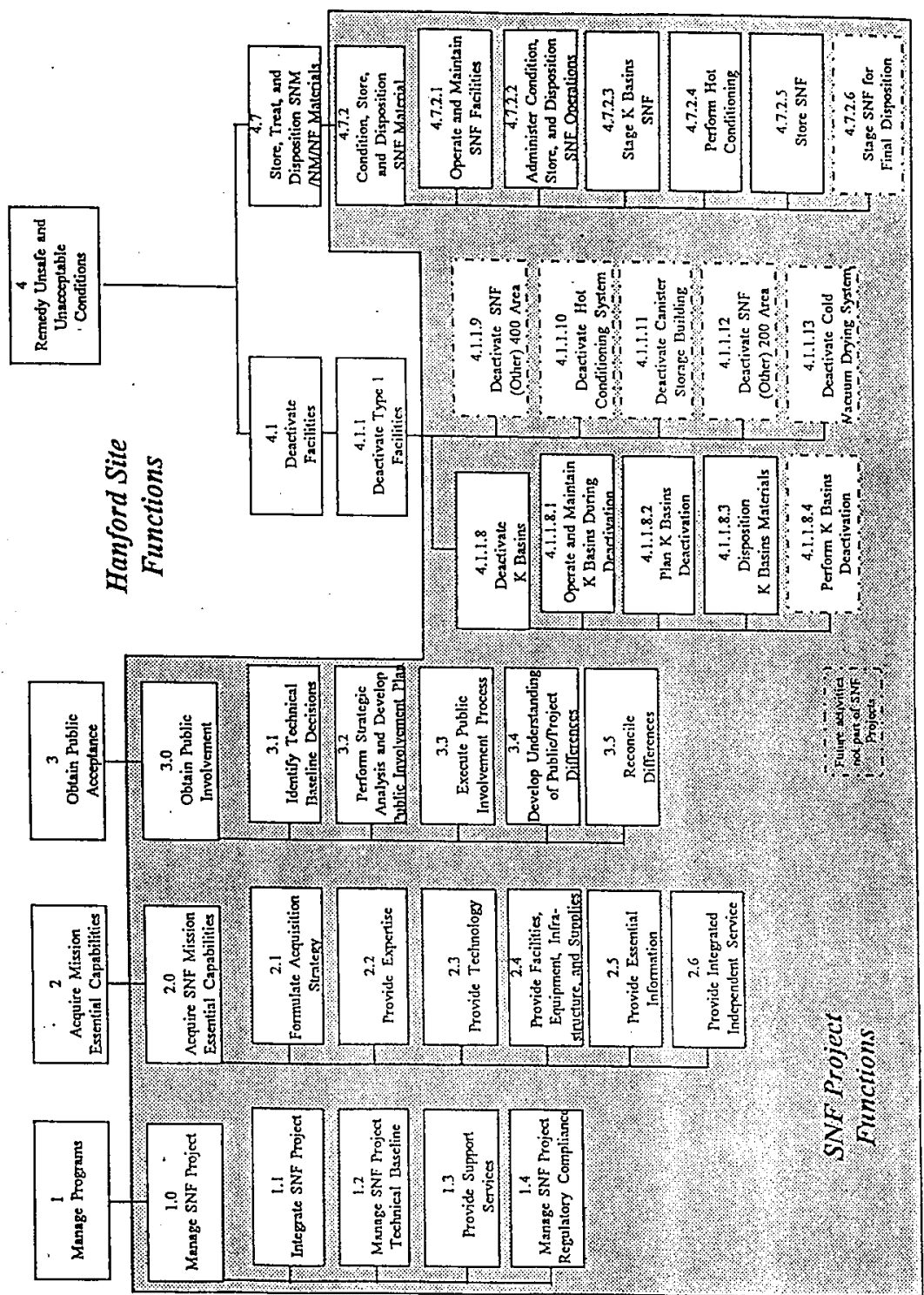




\section{WBS 1.4.1}

HNF-SP-1104, Rev 4

This page intentionally left blank. 
Exhibit 1A

Hanford Site Technical Baseline Data

HNF-SP-1104 Rev 4 
THIS PAGF TNTENTIONALLY i.i. TILANK BL 


\section{TECHNICAL BASELINE}

The technical baseline describes the work (functions) to be accomplished and the technical standards that govern the work. The following inlormation is provided in this section of the Multi-Year Work Plan (MYWP): Project Mission; Project End Point Targets; Summary-Level Forecasting Data (waste type, nuclear material, facility, intrastructure needs); Drivers (key mission and regulatory); Functional. Definitions; Project Life Cycle Requirements; Projecl Issues and Assumptions.

\subsection{PROJECT MISSION}

The Spent Nuclear Fuels (SNF) Projecl supports the Hantord mission to clean up the site by managing and reducing hazards associated with the Hanford spent nuclear fuel inventory, and by managing the effort as a project.

\subsection{PROJECT END POINT TARGETS}

This section identifies the significant end point targets, technical objectives, and safety objectives to be achieved in accomplishing the project mission. This includes general and specific objectives, deliverables, or activities that are essential to the project mission. This information provides an overview of the activities and deliverables that are contained in the schedule section.

\subsubsection{End Point Targets}

Table 1.2.1-1 contains the End Point Targets that are established in the Hantord Mission Direction Document (MDD). They provide the basis for the project mission and are implemented through technical requirements contained in the Hanford Site Technical Baseline.

Table 1.2.1-1 End Point Targets

\begin{tabular}{|c|c|}
\hline 1 & $\begin{array}{l}\text { HOD. Reactors on the Rive. Final, } 1 \\
\text { Spenl hei removed by } 12 / 31 / 99 \text {, K-Basins cleaned sufficient lo transition to } 08 D \text { by } 10 / 31 / 2000\end{array}$ \\
\hline 2 & 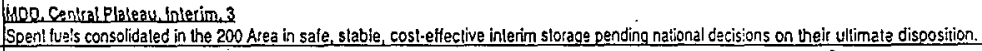 \\
\hline 3 & $\begin{array}{l}\text { MOD. South 600. Final. } 4 \\
\text { Spenilue!s (TRIGA and hight waler reactor) and applicable FFTF fuels removed to } 200 \text { Area. }\end{array}$ \\
\hline 4 & $\begin{array}{l}\text { MoQ. South } 600 \text { Final. } 40 \\
\text { Spenl luals isodium bonded FFTF removed off-site lor final disposition. }\end{array}$ \\
\hline 5 & $\begin{array}{l}\text { MO0. South } 500 \text {. Inteim } 59 \\
\text { Spent fusis (light wales reaclor) removed to inlerim slorage in } 400 \text { Area pending avallability ol } 200 \text { Aisa inis: im slorage. }\end{array}$ \\
\hline
\end{tabular}

\subsubsection{Technical Objectives}

Table 1.2.2-1 contains the Technical Objectives that achieve the end point targets. They are established in various Project specific documents and are implemented through technical requirements contained in the Hanford Site Technical Baseline. 
Table 1.2.2-1 Technical Objectives

\begin{tabular}{|c|c|}
\hline 1 & $\begin{array}{l}\text { SWF Interim Sterage } \\
\text { To provide sale, econornic, and environmentally sound interim siorage for all of the Hanlord Sile's spent nuciear fuel prior to final disposition } \\
\text { ol the malarial. }\end{array}$ \\
\hline 2 & 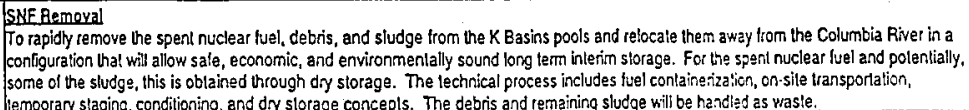 \\
\hline
\end{tabular}

\subsubsection{Safety Objectives}

Table 1.2.3-1 contains the Safety Objectives that are established in the Hantord Mission Direction Document.

Table 1.2.3-1 Salety Objectives

\begin{tabular}{|c|c|}
\hline 1 & $\begin{array}{l}\text { MDD. Reactors on the River. Salety Objective } \$ 38 \\
\text { Provide surveillance and maintenance of the } 1706 \text { KE KELKER Facility. }\end{array}$ \\
\hline 2 & 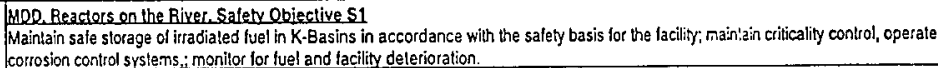 \\
\hline
\end{tabular}

\subsection{SUMMARY-LEVEL FORECASTING DATA}

This section contains forecast information about the project inputs and outputs during the pioject life cycle. The forecast information is an integral part of the technical basis for the planning, scheduling, and budgeting process.

\subsubsection{Waste Type Data (Solid Waste, Tank Waste, Liquid EHfluents, Special Case Waste)}

Table 1.3.1-1 contains the waste (Solid Waste, Tank Waste, Liquid Effluents, Special Case Waste) inventory and volume projection dala. These data are used to track the wasle through generation, transier, receipl, storage, and : disposition. The Project schedule and budget reflect the plans for disposition of waste.

Table 1.3.1-1 Waste Volume Inventory (cubic meters)

\begin{tabular}{|c|c|c|c|c|c|c|c|c|c|c|c|c|c|c|}
\hline & $\begin{array}{l}\text { LLW } \\
\text { (SOL) }\end{array}$ & $\begin{array}{l}\text { ILNW } \\
\text { (SOL) }\end{array}$ & ITU & TRUN & HAZ & HLW & $\begin{array}{l}\text { LLW } \\
\text { LLIO) }\end{array}$ & $\begin{array}{l}\text { LLMW } \\
\text { LLOO) }\end{array}$ & $\begin{array}{l}\text { Industrial } \\
\text { Waste } \\
\text { Wales }\end{array}$ & $\begin{array}{l}\text { Trealed } \\
\text { Liquid } \\
\text { Etiuerni } \\
\end{array}$ & $\left\{\begin{array}{l}\text { Sanitary } \\
(L 10)\end{array}\right.$ & $\begin{array}{l}\text { Sanilary } \\
\text { (SOL) }\end{array}$ & Asbestos & $\begin{array}{l}\text { Specie' } \\
\text { Case } \\
\text { Was!e }\end{array}$ \\
\hline \multicolumn{15}{|l|}{ FY 1997} \\
\hline \multicolumn{15}{|l|}{ Begin Inventory } \\
\hline \multicolumn{15}{|l|}{ Recelvad } \\
\hline Generaled & 379.15 & 1.285 & 0.48 & & & & 75.6 & & & & & & & \\
\hline \multicolumn{15}{|l|}{ Reduced } \\
\hline Translerred & 379.15 & 1.285 & 6.48 & & & & 75.6 & & & & 1 & & & \\
\hline \multicolumn{15}{|l|}{ Discosod } \\
\hline \multicolumn{15}{|l|}{ End Inyentory } \\
\hline \multicolumn{15}{|l|}{ FY 1998} \\
\hline Begin Inventory & & & & & & & & & & & & & & \\
\hline Received & & & & & & & & & & & & & & \\
\hline
\end{tabular}


Table 1.3.1-1 Waste Volume Inventory (cubic meters) (Continued)

\begin{tabular}{|c|c|c|c|c|c|c|c|c|c|c|c|c|c|}
\hline & $\begin{array}{ll}u W W \\
(S O L)\end{array}$ & $\begin{array}{l}\text { LLMWN } \\
\text { (SOL) }\end{array}$ & TRU & TRUM & HAZ & HLW & $\mid \begin{array}{l}L L W \\
(L I O)\end{array}$ & $\begin{array}{l}\text { LLNW } \\
\text { (LIO) }\end{array}$ & $\begin{array}{l}\text { Industrial } \\
\text { Waste } \\
\text { Water }\end{array}$ & $\begin{array}{l}\text { Trealed } \\
\text { Liquid } \\
\text { Entuent }\end{array}$ & $\begin{array}{l}\text { Sanilary } \\
(L \perp Q)\end{array}$ & $\begin{array}{l}\text { Sanitary } \\
\text { (SOL) }\end{array}$ & 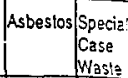 \\
\hline Generated & 677.474 & 1.285 & 67.42 & & & & 643.0 & & & & & & \\
\hline Reduced & & & & & & & & & & & & & \\
\hline Transferred & 677.474 & 1,285 & 67.42 & & & & 643.0 & & & & & & \\
\hline Disposed & & & & & & & & & & & & & \\
\hline End Inventory & & & & & & & & & & & & $\ldots$ & \\
\hline$F Y 1999$ & & & & & & & & & & & & & \\
\hline Begin loyentory & & & & & & & & & & & & & \\
\hline Received & & & & & & & & & & & & & 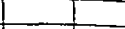 \\
\hline Generated & 539.514 & 1.285 & 3.24 & & & & 681.0 & & & & & & \\
\hline Reduced & & & & & & & & & & & - & & \\
\hline Transferred & 639.514 & 1.285 & 3.24 & & & & 681.0 & & & $\therefore$ & & & \\
\hline Disposed & & & & & & & & & & & & & \\
\hline End inventory & & & & & & & & & & & & & \\
\hline$F Y 2000$ & & & & & & & & & & & & & \\
\hline Begin Inventory & & & & & & & & & & & & & \\
\hline Received & & & & & & & & & & & & & \\
\hline Generaled & 294.098 & 1.285 & & & & 927.4 & 151.2 & & & & & & 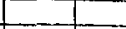 \\
\hline Reduced & & & & & & & & & & & & & \\
\hline Translemed & 294.098 & 11.285 & & & & 927.4 & 1151.2 & & & & & & \\
\hline Disposed & & & & & & & & & & & & & \\
\hline End Inventory & & & & & & & & & & & & & 5 \\
\hline FY 2001 & & & & & & & & & & & & & \\
\hline Begin Invenlory & & & & & & & & & & & & & $E$ \\
\hline Received & & & & & & & & & & & & & \\
\hline Genesated & & & & & & 397.4 & 95.0 & & & & & & \\
\hline Reduced & & & & & & & & & & & & & \\
\hline Translemed & & & & & & 397.4 & 95.0 & & & & & & \\
\hline Disposed & & & & & & & & & & & & & \\
\hline End inveniory & & & & & & & & & & & & & \\
\hline FY 2002 & & & & & & & & & & & & & \\
\hline Beain Inyentory & & & & & & & & & & & & & \\
\hline Received & & & & & & & & & & & & & \\
\hline Generated & & & & & & & 2271.0 & & & & & & \\
\hline Reduced & & & & & & & & & & & & & \\
\hline Translerred & & & & & & & 2271.0 & & & & & & \\
\hline Disposed & & & & & & & & & & & & & \\
\hline End Inventory & & & & & & & & & & & & . & \\
\hline FY 2003 & & & & & & & & & & & & & \\
\hline Begin Inveniory & & & & & & & & & & & & & \\
\hline Received & & & & & & & & & & & & & \\
\hline Generated & & & & & & & 2271.0 & & & & & & \\
\hline Reducod & & & & & & & & & & & & & \\
\hline Iransterred & & & & & & & 2271.0 & & & & & & \\
\hline Disposed & & & & & & & & & & & & & \\
\hline End inventory & & & & & & & & & & & & & \\
\hline$Y_{2} 2004$ & & & & & & & & & & & & & \\
\hline Beoin Inrentory & & & & & & & & & & & & & \\
\hline Received & & & & & & & & & & & & & \\
\hline Generated & & & & & & & 4542.0 & & & & & & \\
\hline Reduced & & & & & & & & & & & & & \\
\hline Translered & & & & & & & 4542.0 & & & & & & \\
\hline Disposad & & & & & & & & & & & & & \\
\hline End Invenlor & & & & & & & & & & & & & \\
\hline
\end{tabular}




\subsubsection{Nuclear Materials (Special Nuclear Materials, Nuclear Fuel, Cesium capsules, Strontium capsules)}

Table 1.3.2-1 contains the nuclear materials (Special Nuclear Materials, Nuclear Fuel, Cesium capsules, Strontium capsules) inventory and projection data. These data are used to track the nuclear materials through transier, receipt, storage, and disposition. The Project schedule and budget reflect the plans for disposition of nuclear malerials.

Table 1.3.2-1 Nuclear Materials Inventory

\begin{tabular}{|c|c|c|c|c|c|}
\hline & $\begin{array}{l}\text { PUHEU } \\
(K O)\end{array}$ & $\begin{array}{l}\text { |rradiated Fuel } \\
\text { (MTHM) }\end{array}$ & $\begin{array}{l}\text { Cs Capsules } \\
\text { (Number ol cagsules) }\end{array}$ & $\begin{array}{l}\text { Si Capsules } \\
\text { (Number of Ceossites) }\end{array}$ & $\begin{array}{l}\text { Unirradiated Uranium } \\
(M T-U)\end{array}$ \\
\hline \multicolumn{6}{|l|}{ FY 1997} \\
\hline Begin Inventory & & 2103.0 & & & \\
\hline \multicolumn{6}{|l|}{ Received } \\
\hline \multicolumn{6}{|l|}{ Generated } \\
\hline \multirow{2}{*}{\multicolumn{6}{|c|}{$\begin{array}{l}\text { Reduced } \\
\text { Transletred }\end{array}$}} \\
\hline & & & & & \\
\hline \multicolumn{6}{|l|}{ Disposed } \\
\hline End Inveniory & & 2103.0 & & & \\
\hline \multicolumn{6}{|l|}{ YY1998 } \\
\hline Begin Inventory & & 2103.0 & & & \\
\hline \multicolumn{6}{|l|}{ Peceived } \\
\hline \multicolumn{6}{|l|}{ Generaled } \\
\hline \multicolumn{6}{|l|}{ Reduced } \\
\hline Transterred & & 900.0 & & & \\
\hline \multicolumn{6}{|l|}{ Disposed } \\
\hline End Inventory & & 1203.0 & & & \\
\hline \multicolumn{6}{|l|}{ Fr1999 } \\
\hline Begin Inveniony & & 11203.0 & & & \\
\hline \multicolumn{6}{|l|}{ Received } \\
\hline \multicolumn{6}{|l|}{ Generaled } \\
\hline \multicolumn{6}{|l|}{ Reduced } \\
\hline Translerred & & 9080.0 & & & \\
\hline \multicolumn{6}{|l|}{ Disposed } \\
\hline End inventory & & 123.0 & & & \\
\hline \multicolumn{6}{|l|}{ EY 2000} \\
\hline Begin Inventory & & 123.0 & & & \\
\hline Received & & 7.49 & & & \\
\hline \multicolumn{6}{|l|}{ Generaled } \\
\hline \multicolumn{6}{|l|}{ Reduced } \\
\hline Transferted & & 120.0 & & & \\
\hline \multicolumn{6}{|l|}{ Disposed } \\
\hline End Invenlory & & 10.49 & & & \\
\hline \multicolumn{6}{|l|}{ FY 2001 } \\
\hline Begin livenlony & & 10.49 & & & \\
\hline Received & & 21.0 & & & \\
\hline \multicolumn{6}{|l|}{ Generated } \\
\hline \multicolumn{6}{|l|}{ Reduced } \\
\hline \multicolumn{6}{|l|}{ Transterred } \\
\hline Disposed & & & & & \\
\hline End Inventory & 1 & 61.5 & & & \\
\hline
\end{tabular}

\subsubsection{Facilities (Excess, Deactivated)}


Tabte 1.3.3-1 contains the facility forecasting data (facility deactivation, decontamination and decommissioning, and closure). The facility data are used to track the facility through acquisition, operations and maintenance, and disposal. The Projecl schedule and budget reflect the plans for disposition of excess and deactivated facilities.

Table 1.3.3-1 Facility Inventory

\begin{tabular}{|c|c|c|c|c|c|c|c|}
\hline Complex & Facilly & Facility Description & Acquisition Project & MSO Projecl & $\begin{array}{l}\text { Deactivation } \\
\text { Project }\end{array}$ & DSD Project & Ciosure Projecl \\
\hline FFTF & $432 \mathrm{~A}$ & $\begin{array}{l}\text { ISA COVERED } \\
\text { EQUIPMENT STORAGE }\end{array}$ & & $\begin{array}{l}\text { Advanced } \\
\text { Reaclots }\end{array}$ & Spent Nuclear Fue! & & \\
\hline FFTF & 4716 & $\begin{array}{l}\text { WTERIN STORAGE } \\
\text { AREA }\end{array}$ & & $\begin{array}{l}\text { Advanced } \\
\text { Reaclors }\end{array}$ & Spenil Nuclear Fue: & & \\
\hline $\begin{array}{l}\text { ERC } \\
\text { SNF }\end{array}$ & $105 \mathrm{KE}$ & $\begin{array}{l}\text { Reaclor Building } \\
\text { FUEL STORAGE BASIN }\end{array}$ & & Spent Nucleat Fuel & & $\begin{array}{l}\text { Ervivnmental } \\
\text { Bistioration }\end{array}$ & $\begin{array}{l}\text { Environmental } \\
\text { Resloration }\end{array}$ \\
\hline $\begin{array}{l}\text { EAC } \\
\text { SNF }\end{array}$ & $105 \mathrm{~kW}$ & $\begin{array}{l}\text { Reactor Building } \\
\text { FUEL STORAGE BASIN }\end{array}$ & & Spent Nuciear Fuel & & $\begin{array}{l}\text { Envivnmental } \\
\text { Anessioration }\end{array}$ & $\begin{array}{l}\text { Environmental } \\
\text { Resiosation }\end{array}$ \\
\hline SNF & $119 \mathrm{KE}$ & $\begin{array}{l}\text { 119-KE EXHAUST AIR } \\
\text { SAMPLING BLOG }\end{array}$ & & Spenl Nuciear Fuel & & $\begin{array}{l}\text { Envionmental } \\
\text { Pestoration }\end{array}$ & \\
\hline SNF & $1614 \mathrm{KE}$ & $\begin{array}{l}\text { ENVIRONMENTAL } \\
\text { MONITORING STATION }\end{array}$ & & Spenl Nucjear Fuel & & $\begin{array}{l}\text { Enxionmental } \\
\text { Pि=sioviation }\end{array}$ & . \\
\hline SNF & $165 \mathrm{KE}$ & KE Power Control Building & & Spent Nucleat Fuel & & $\begin{array}{l}\text { Envionmental } \\
\text { Anestoration }\end{array}$ & \\
\hline SNF & 166AKE & Oil Storage Facility & & Spent Nuclear Fuel & & $\begin{array}{l}\text { Entionmental } \\
\text { fatsoralion }\end{array}$ & \\
\hline SNF & $1705 \mathrm{KE}$ & $\begin{array}{l}\text { EFFLUENT WATEA } \\
\text { TREATMENT PILOI PLA }\end{array}$ & & Spent Nuclear Fuel & & $\begin{array}{l}\text { Ent: innental } \\
\text { Aus'sralion }\end{array}$ & \\
\hline SNF & $1713 \mathrm{KE}$ & SHOP EUILDING & & Spent Nuclear Fuel & & $\begin{array}{l}\text { Entronmenial } \\
\text { Gestoration }\end{array}$ & \\
\hline SNF & $1713 \mathrm{KW}$ & WAREHOUSE & & Spenl Nuclear Fue & & $\begin{array}{l}\text { Envionmental } \\
\text { Risicration }\end{array}$ & \\
\hline SNF & 17I\&KE & $\begin{array}{l}\text { OIL AND PAINT } \\
\text { STORAGE SHED }\end{array}$ & & Spenil Nuclear Fuel & & $\begin{array}{l}\text { Envionmental } \\
\text { Res'uration }\end{array}$ & \\
\hline SNF & $1714 \mathrm{KW}$ & KW Warehouse & & Spent Nuclear Fue? & & $\begin{array}{l}\text { Entwinmenta! } \\
\text { Restoration }\end{array}$ & \\
\hline SNF & $1717 \mathrm{~K}$ & $\begin{array}{l}\text { MAINTENANCE } \\
\text { TRANSPORTATION } \\
\text { SHOP BU }\end{array}$ & & Spent Nuclear Fuel & & $\begin{array}{l}\text { Encionmenta! } \\
\text { Rus'oration }\end{array}$ & \\
\hline SNF & I81KE & RIVER PUMP HOUSE & & Spenil Nuclear Fugl & & $\begin{array}{l}\text { Enrionmenta! } \\
\text { Res'suation }\end{array}$ & \\
\hline SNF & 183.1KE & HEADHOUSEICHLORINE & & Spent Nuclear Fuel & & $\begin{array}{l}\text { Enrizmmenlal } \\
\text { AEs'gralion }\end{array}$ & \\
\hline SNF & $183.1 \mathrm{~kW}$ & CHLORINE VAULT & & Spent Nuclear Fuel & & $\begin{array}{l}\text { Entunmental } \\
\text { Estoration }\end{array}$ & \\
\hline SNF & 183-2KE & KE Sedimentation Basins & & Spent Nuclear Fueil & & $\begin{array}{l}\text { Emironmental } \\
\text { Ris:viation }\end{array}$ & \\
\hline SNF & $183.3 \mathrm{KE}$ & KE Filter Basin & & Speni Nuclear Fua: & & $\begin{array}{l}\text { Ensironmental } \\
\text { Ais'sration }\end{array}$ & \\
\hline SNF & $183-4 \mathrm{KE}$ & KE Reservoir \& Clearwelis & & Spenil Nucleat Fues & & $\begin{array}{l}\text { Erivonnental } \\
\text { hiscoration }\end{array}$ & \\
\hline SNF & $183.5 \mathrm{KE}$ & HME FEEDER BUILDING & & Spent Nuclear Fuel & & $\begin{array}{l}\text { Entisnmental } \\
\text { Extratation }\end{array}$ & \\
\hline SNF & 183-6KE & LLHE FEEDER BUILDING & & Spent Nuclear Fuel & & $\begin{array}{l}\text { Enotonmental } \\
\text { Res'aralion }\end{array}$ & \\
\hline SNF & 183KE & $\begin{array}{l}\text { FILTER PLANT HEAD } \\
\text { HOUSE, CHLORINE }\end{array}$ & & Spent Nuclear Fue: & & 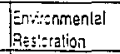 & \\
\hline SNF & $1908 \mathrm{~K}$ & OUTFALL & & Spent Nuclear Fueil & & $\begin{array}{l}\text { Ex:-ronmenta! } \\
\text { PE:zration }\end{array}$ & \\
\hline SNF & $1908 \mathrm{KE}$ & $\begin{array}{l}\text { EFFLUENT WATER } \\
\text { MONITORING STATION }\end{array}$ & & Spenl Nucleat Fue! & & $\begin{array}{l}\text { Enitonmental } \\
\text { Pzidgation }\end{array}$ & \\
\hline SNF & $190 \mathrm{KE}$ & MAIN PUMP HOUSE & & Spent Nuclear Fuz: & & $\begin{array}{l}\text { Eritionmental } \\
\text { Fiesisration }\end{array}$ & \\
\hline
\end{tabular}




\subsubsection{Infrastructure (Power, Steam, Water, Roads, Railroad, Sanitary Waste)}

This section contains the forecasting data for infrastructure support. These data are used to ensure the required infrastructure is available when it is needed. Table $1.3 .4-1$ provides the nominal, best estimale of infrastructure needs. Table 1.3.4-2 provides an estimate of the maximum probable need. The Project schedule and budget reflect the services (infrastructure) that are necessary to achieve the project mission.

Table 1.3.4-1 Infrastructure Requirements - Average Demand

\begin{tabular}{|c|c|c|c|c|c|c|c|c|c|c|c|}
\hline Infrasinucture Type. & 1507 & 1998 & 1999 & 2000 & 2001 & 2002 & 2003 & 2004 & $20 \%$ & $200 \div 2070$ & Units \\
\hline \multicolumn{12}{|l|}{ AllocaredLand } \\
\hline Analylical Labocalon Services & 350,0 & 6700.0 & 57000 & 3700.0 & 6700.0 & & & & & & SAMPLESTA \\
\hline Bioassay and Dosimein Servizes & $1 \leqslant 000$ & $1+9000$ & 190000 & 19000 & 1600.0 & & & & & & SANPLESTRA \\
\hline Gialogical Laboraton Sevices & 4930 & 4930 & 493.0 & 493.0 & 493.0 & & & & & & SAMIPLESNAR \\
\hline Building blazinienance & $1 \leqslant 000$ & 7200.0 & 0.0 & 0.0 & 0.0 & & & & & & SO FT. \\
\hline Clean Laundry & 9740000 & 874000.0 & 3740000 & 974000.0 & 1874000.0 & & & & & & LESNA \\
\hline Custodial Senices & 220000 & 22000.0 & 200000 & 26000.0 & 26000.0 & & & & & & $50 \mathrm{FT}$ \\
\hline Dala (HLAN) Transmission & 3550 & 365.0 & 305.0 & 365.0 & 365.0 & & & & & & No OFPCs \\
\hline \multicolumn{12}{|l|}{ Qeveloonent Laboratory Senicas } \\
\hline Electricity & 14,0 & 24.0 & 340 & 29.0 & 29.0 & & & & & & SWY-HANA \\
\hline Energy Management Services & 0.0 & 0.0 & 10.0 & 0.0 & 0.0 & & & & & & PROJECTSNR \\
\hline $\begin{array}{l}\text { Environmental Mciecular Science Lab } \\
\text { Services }\end{array}$ & 0.0 & 0.0 & 0.0 & 0.0 & 0.0 & & & & & & SYR \\
\hline Fab Shoo Sevices & 10000 & 250.0 & 1000 & 100.0 & 100.0 & & & & & & LASOR-HAYR \\
\hline Guaranleed Ride Home & 20.0 & 50.0 & 500 & 500 & 50.0 & & & & & & PASSENGERSNA \\
\hline Hantord Road Sys. Heawy Treilic & 50.0 & 340.0 & $3 \div 0.0$ & 700 & 10.0 & & & & & & IRUCK LOADSYYA \\
\hline Heaw Equipment & 00 & 0.0 & 0.0 & 10.0 & 0.0 & & & & & & EOUIP-DAYSYYR \\
\hline Heavy Trucks & 5000 & 240.0 & 34000 & 3400.0 & 3400.0 & & & & & & VEHICLE-HANA \\
\hline in- Field Laboralon Services & 160 & 6.0 & 160 & 6.0 & 66.0 & & & & & & SAMIPLESNA \\
\hline \multicolumn{12}{|l|}{ Industrial Hygiene Servicas } \\
\hline Lihing (Cranes) & 160.0 & 60.0 & 500 & 60.0 & 600 & & & & & & CRANE DAYSNA \\
\hline \multicolumn{12}{|l|}{ Mion-rad Siandards (Callowalior.s) } \\
\hline Otfice Soace [Leased) & bo & 0.0 & 0,0 & 0.0 & 0.0 & & & & & & SO.FT. \\
\hline OHice Soace Inlias!nucture Oaned) & 114000 & 7200.0 & 0.0 & 0.0 & 0.0 & & & & & & SO.FT. \\
\hline \multicolumn{12}{|l|}{ Otice Soace (Piogram Owneol } \\
\hline Pager Service & 1322.0 & 322.0 & 3220 & 322.0 & 312.0 & & & & & & NOO. OF PAGERS \\
\hline Polabie Waler & 6.7 & 0.7 & 07 & 0.7 & 0.7 & & & & & & MGAUNR \\
\hline Radioaclive Standards (Calitrations) & 1300.0 & 13000 & 13000 & 1300.0 & 350.0 & & & & & & CALIBRATIONSNR \\
\hline \multicolumn{12}{|l|}{ Bail Transpotition } \\
\hline Rิaw Wal & bo & 100 & 0.0 & 0.0 & 0.0 & & & & & & :34: \\
\hline Sedanshigh! Trucks & 310 & 37.0 & 32.0 & 210 & 21.0 & & & & & & NO. OF VEHICLES \\
\hline Steam & bo & 0.0 & 0.0 & 0.0 & 10.0 & & & & & & LLSSNA \\
\hline Slorage Space inl fastucture Omeol & 960 & 10000.0 & 10000 & 0.0 & 0.0 & & & & & & SO.FT. \\
\hline Slorage Space Reased & 0.0 & 00 & 0.0 & To.0 & 0.0 & & & & & & So.Fi. \\
\hline \multicolumn{12}{|l|}{ Sloraoe Soace (Program Ownet) } \\
\hline Taxi Service & 6000 & 6000 & 16000 & 600.0 & 6000 & & & & & & PASSENGEFSYYR \\
\hline Video Communicalion & 60.0 & 60.0 & 160.0 & 60.0 & 60.0 & & & & & & HAYR \\
\hline Voice Teleghong, Communistion & 350.0 & 430.0 & 年 & 6300 & 430.0 & & & & & & NO OF PHONES \\
\hline
\end{tabular}

Table 1.3.4-2 Infrastruclure Requirements-Peak Demand

\begin{tabular}{|c|c|c|c|c|c|c|c|c|c|c|c|}
\hline Intra struclure Tyes & 1997 & 1992 & 1999 & 2000 & 2001 & 2002 & 2003 & 2004 & $2 \%$ & $2000-2070$ & Units \\
\hline \multicolumn{12}{|l|}{ Allocalad Land } \\
\hline Analydical Laboratony Servizes & 63000 & 6700.0 & 6700.0 & 6700.0 & 6700.0 & & & & & & SAUPLESTH \\
\hline Bioassay and Dosinely Serizes & 15000 & 19000 & 15000 & 1950.0 & 1600.0 & & & & & & SAMPLESTRR \\
\hline Biglogical Laboraloy Servises & 1493.0 & 492.0 & 433.0 & .93 .0 & 493.0 & & & & & & SAMPLESYR \\
\hline Building Mlaintenance & 124000 & 72000 & 00 & 0.0 & 0.0 & & & & & & SO.FT. \\
\hline Oean Laundiy & 13740000 & 3740000 & 574200.0 & 974000.0 & 374000.0 & & & & & & LBSTA \\
\hline Cuslodal Services & 23000.0 & 220000 & 260000 & 26000.0 & 26000.0 & & & & & & SOFT. \\
\hline Oala (HLAN) Transmission & 73550 & 365.0 & 335.0 & 3650 & 305.0 & & & & & & No. OFPCs \\
\hline \multicolumn{12}{|l|}{ Develogment Laboratory Serrices } \\
\hline Electricity & co & 00 & 0.0 & 0.0 & 0.0 & & & & & & SWM-HAYA \\
\hline
\end{tabular}


Table 1.3.4-2 Infrastructure Requirements - Peak Dernand (Continued)

\begin{tabular}{|c|c|c|c|c|c|c|c|c|c|c|c|}
\hline Iniastructure Tree & 1997 & 1999 & 1999 & 2000 & 2001 & 2002 & 2003 & 2004 & 2005 & $2006-2070$ & Unils \\
\hline Energy Managemenl Services & 0.0 & 00 & bo & 0.0 & 0.0 & & & & & & PROJECTSYA \\
\hline $\begin{array}{l}\text { Environmental Molecular Sojenca Lab } \\
\text { Senices }\end{array}$ & 0.0 & 0.0 & 0.0 & 0.0 & 0.0 & & & & & & SYR \\
\hline Fab Strod Strvices. & 10.0 & 0.0 & 0.0 & 0.0 & 0.0 & & & & & & LABOA.HANA \\
\hline Guaranteed Ride Home & 0.0 & 0.0 & 0.0 & 0.0 & 0.0 & & & & & & PASSENGERSYA \\
\hline Hanlord Roas Srs. Heaw Tralic & 50.0 & 340.0 & 340.0 & 70.0 & 10.0 & & & & & & TRUCKLOADSNA \\
\hline Heavy Ecuipmen! & 0.0 & 0.0 & 0.0 & 10.0 & 0.0 & & & & & & EOUIP.DAYSNA \\
\hline Heavy Tnicks & 87600 & 2760.0 & 8760.0 & 8760.0 & 8760.0 & & & & & & VEHICLE-HRNR \\
\hline in-Fieid Laboratory Services & 5.0 & 6.0 & 60 & 5.0 & 5.0 & & & & & & SALIPLESNA \\
\hline \multicolumn{12}{|l|}{ Industrial Hygiene Sevices } \\
\hline Lithing (Cranes) & 60.0 & 60.0 & 60.0 & 00.0 & 60.0 & & & & & & CRANE DAYSNR \\
\hline \multicolumn{12}{|l|}{ Non-rad Slandards (Catbralions) } \\
\hline Othice Space [Leased & 0.0 & 0.0 & 0.0 & 0.0 & 0.0 & & & & & & SO. \\
\hline Otice Space inirastiveture Omnath & $1<4000$ & 7200.0 & 0.0 & 0.0 & 0.0 & & & & & & So. FT. \\
\hline Otice Soace (Prooram Omaol. & & & & & & & & & & & - \\
\hline Pager Service & 322.0 & 13220 & 3220 & 322.0 & 312,0 & & & & & & NO. OF PAGERS \\
\hline Polable Wa!er & 0.0 & 0.0 & 00 & 0.0 & 0.0 & & & & & & MGAYYA \\
\hline Radioactive Standards ICafibiations]. & 800.0 & 1300,0 & 1300.0 & 1300.0 & 850,0 & & & & & & CALIBRATIONSNA \\
\hline \multicolumn{12}{|l|}{ Aail Transpontation } \\
\hline Raw Waler & 0.0 & 0.0 & 0.0 & 0.0 & 0.0 & & & & & & SIGALYA \\
\hline Sedanslichi Trueks & 31.0 & 37.0 & 32.0 & 210 & 210 & & & & & & NO OF VEHICLES \\
\hline Steamt & 10.0 & 0.0 & 0.0 & 0.0 & 0.0 & & 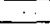 & & & & LBSNA \\
\hline Slorage Soace finlrastruclure Owneo & 100 & 10000.0 & 10000.0 & 0.0 & 0.0 & & & & & & SO.FT. \\
\hline Storage Space (Leased) & 0.0 & 0.0 & 00 & 0.0 & 0.0 & & & & & & 50.F. \\
\hline \multicolumn{12}{|l|}{ Storage Space (Proo:am Owined) } \\
\hline Taxi Service & 0.0 & 0.0 & 0.0 & 0.0 & 0.0 & & & & & & PASSENGEASYA \\
\hline Video Communication & 60.0 & 60.0 & 60.0 & 60.0 & 60.0 & & & & & & HRYล \\
\hline Voice (Teleohone) Communication & 350.0 & 430.0 & 430.0 & 430.0 & 4300 & & & & & & NO OF PMONES \\
\hline
\end{tabular}

\subsection{DRIVERS}

This section identifies the documents that are sources of project requirements, including Key and Regulatory Drivers.

\subsubsection{Key Drivers}

Table 1.4.1-1 lists the source documents that tend to drive the project mission (e.g. Mission Direction Document, Tri-Party Agreement).

Table 1.4.1-1 Key Drivers

\begin{tabular}{|c|c|}
\hline Name & Thie \\
\hline EHI Letter $0162 \cdot 15$ & Memorandum of Underslanding for Saleguards and Security of Nuclear Melerial at N Reacloi, June 15, 1995 \\
\hline ONFSB 94.1 & Dseiense Nuciear Facitities Salety Board Recommendalion 94.1 \\
\hline $\begin{array}{l}\text { DOE Leller } \\
95 \text {-AMW.003 }\end{array}$ & Approval of Spenl Nuclear Fuel (SNF) Path Forward Recommendation, Febtuary 14, 1995 \\
\hline $\begin{array}{l}\text { DOE Leiter } \\
\text { 95.NMO-071 }\end{array}$ & Approval ol X Basins Sludge Disposition Stralegy, June 13, 1995 \\
\hline $\begin{array}{l}\text { DOE Leller } \\
\text { 95-NMD.090 }\end{array}$ & Canister Storage Building (CSB) Design Lite Extension, June 19, 1995 \\
\hline DOE Leller 95.57 & $\begin{array}{l}\text { Ofice of Civilian Radioactive Wasle Mangement (OCRW), "Quality Assurance Requirements and Désziplion (QARD) [RW-033PJ", July } \\
12,1995\end{array}$ \\
\hline
\end{tabular}


Table 1.4.1-1 Key Drivers (Continued)

\begin{tabular}{|c|c|}
\hline Name & Tille \\
\hline DOE Leller 95-SFD-113 & $\begin{array}{l}\text { Spen! Nuclear Fuel (SNP) Mukiple Canisler Overpack (MCO) Conloumance lo National Mutti.Purose Canisler (MPC) Subsystem, July } \\
17,1995\end{array}$ \\
\hline DOE Lefter 95.SFD.132 & Approval ol Spent Nucleat Fuel (SNF) Path Fonward Recommendation, Juty 31, 1995 \\
\hline DOE Leller 95.SFD-167 & Implementation ol the K Easins Spent Nuclear Fuel Project (SNFP) Regulatory Pollicy, Seplemte: 12, 1295 \\
\hline $\begin{array}{l}\text { DOE Owned Spent } \\
\text { Nuclear Fuel Sirategic } \\
\text { Plan }\end{array}$ & $\begin{array}{l}\text { DOE Owned Spent Nuclear Fue! Strategic Plan, U.S. Department of Energy Ofice of Envirormential Fastoralion and Wasle } \\
\text { Managemenl (EM-37). Decernbe! } 1994\end{array}$ \\
\hline DOE/AL.93.10? & Fiscal Year 1995 Hanlord Mission Plan Volume 1, Site Guldance \\
\hline DOERRL-96.14 & Uodeted Drath Mission Direction Document June 1996 \\
\hline SNF-RD-PM-001 Rev 0 & Spen! Nuclear Fuel Program Requiremen!s Document, Revision 0 \\
\hline Tri-Party Agieement & Hanlord Federal Facility Aoreemenl and Consent Order: $89-10$, Rev. 1 \\
\hline WHC-SP-1144 & Spent Nuclaar Fuel Projecl K Ba sins Pa:h Forward Acquisition Stralegy, Rev. F, Drall, December 1994 \\
\hline
\end{tabular}

\subsubsection{Other Drivers}

Table 1.4.2-1 contains the source documents that must be followed as the project mission is accomplished.

Table 1.4.2-2 Other Drivers

\begin{tabular}{|c|c|}
\hline Name & Tille \\
\hline 10 CFR 1017 & Identification and Proleclion of Unclassified Conlroiled Nucleat Inlormation \\
\hline 10 CFR 1021 & NEPA inglemenling Procedures \\
\hline $10 \mathrm{CFR} 1022$ & Compliance with FloodplainWellands Environmenlat Review Regutitements \\
\hline $10 \mathrm{CFA} 20$ & Slandards for Proleclion Agains! Rejdialion \\
\hline $10 \mathrm{C} F \mathrm{~F} 435$ & Energy Conservation Voluntary Pertormance Siandards for New Buildings; Aendatory lo: Fejarel Eutings \\
\hline 10 CFR 435 & Federal Energy Management and Planning Programs; Lite-Cycle Cos! Methodology and Procestyres \\
\hline 10 CFR 50 & Domestic Licensing of Production and Utilization Facilities \\
\hline 10 CFP 71 & Packaging and Transportation of Radioactive Material \\
\hline 10 CFR 71.55 & Packaqing and Transportalion of Redioaclive Malerial: General Requiremenls lor Fissile Malerrial Paxizgas \\
\hline 10 CFR 72 & Licensing Requitements lor the independent Storage of Spent Nuclear Fuel and High-Leval Radioasliva Wasle \\
\hline 10 CFR 830 & Nuclear Salety Management, 4/5/94 \\
\hline 10 CFR 830.120 & Quelity Assurance Requitrements \\
\hline 10 CFR 835 & Oscupational Padialion Prolection \\
\hline 16 U.S.C. $470 \mathrm{aa}-47011$ & Conservalion; Archaeological Resources ACl \\
\hline 16 U.S.C. SEcion 1531 & Conservation; Congressional Findings and Declaration ol Purposes and Policy \\
\hline 16 U.S.C. Stelion $6 \hat{g} 9$ & Conservation: Preservalion of historical and Archeological Dala Threalenes by Dam Consluction er Fyetations of Terrain \\
\hline 16 USC 651 et seq & Proleclion and Conservation of Wildirle \\
\hline 25 USC 3001 el sea & Native American Graves Prolection and Repatriation Act \\
\hline 29 CFA 1504 & Recording and Reoorling Occupational Injuries and lliness \\
\hline 29 CFR 1910 & Oecuoational Salety and Healih Standards \\
\hline
\end{tabular}


Table 1.4.2-2 Other Drivers (Continued)

\begin{tabular}{|c|c|}
\hline Name & Title \\
\hline$\otimes$ CFA 1925 & Salely and Healih Requlalions During Consinuction \\
\hline 29 USC 651 el seq & Occupational Salety and Health \\
\hline 33 CFR 153 & Control ol Pollulion by Oil and Hazardous Substances, Discharoe Removal \\
\hline 33 CFR 322 Section 10 & Penrils for Siructures or Work in or Affecting Navioable Walers of the Unilted Stales \\
\hline 33 CFR 325 & Processing of Departmenl of the Army Permils \\
\hline 33 USC 1251 el seg & Waler Pollution and Prevention Conlrols: Concressional Declaralion ol Goals and Policy \\
\hline 40 CFR 110 & Discharge ol Oil \\
\hline 40 CFR 116 & Designation of Hazardous Substances \\
\hline 40 CFA 117 & Deleiminalion of Reportable Ouantities for Hazardous Substances \\
\hline $40 \mathrm{CrR} 122$ & EPA Administared Pemit Programs: The National Pollulanl Discharge Elimination Syslem Pemirs. \\
\hline 40 CFR 129 & Toxic Pollulant Effivenl Standards \\
\hline 40 CFR 141 & National Primary Drinking Waler Regulations \\
\hline 40 CFR 144 & Underaround Iniection Conlrol Program \\
\hline 40 CFR 146 & Underoround linjeclion Control Program: Crileria and Slandards \\
\hline 40 CFR 148 & Hazardous Wasle Injection Restriction \\
\hline $\begin{array}{l}40 \text { CFR } 1500 \text { through } \\
1508\end{array}$ & Council on Environmental Quality, NEPA, EIS \\
\hline 40 CFA 191 & $\begin{array}{l}\text { Environmental Radialion Protection Standards for Management and Disposal ol Spenl Nuclear Futl, High-Level and Transuranic } \\
\text { Radioaclive Wasles }\end{array}$ \\
\hline 40 CFR 241 & Guidelines tor the Land Disposal ol Solid Wasle \\
\hline 40 CFA 244 & Solid Was!e Managemen! Guidelines for Beverage Containers \\
\hline 40 CFR 247 & Guidelines for Procurement of Producls that Contain Recycled Materials \\
\hline 40 CFR 248 & Guideline for Federal Procurement of Building Producls Containing Recovered Malerials \\
\hline 40 CFR 249 & Guideline lor Federal Procurement ol Cernent and Concrele Containing Fly Ash \\
\hline 40 CFA 250 & Guideline lor Federal Procurement ol Paper and Paper Producls Containing Recovered Materials \\
\hline 40 CFR 252 & Guideline lor Federal Procuternent ol Lubricating Oils Conlaining Rafined Dil \\
\hline 40 CFR 253 & Guivelina lor Federat Procurement of Retread Tires \\
\hline 40 CFR $25 ?$ & Criletia lor Classification ol Solid Wasle Disposal Facilities and Piaclices \\
\hline 40 CFA 260 & Hazardous Waste Managoment System: General \\
\hline 40 CFA 261 & Identificalion and Lisling ol Hazardous Waste \\
\hline 40 CFR 262 & Standards koplicable 10 Generalors o! Hazardous Wasle \\
\hline 40 CFR 263 & Slandards Apolicable lo Transponters of Hazardous Waste \\
\hline 40 CFR 264 & Standards for Owmers and Operalors of Hazardous Wasle Trealment, Storage, and Disposal Fasirie: \\
\hline 40 CFR 265 & Interim S! zius Standards for Oxners and Operators of Hazardous Waste Treaiment, Storage, znd Q soosal Facillies \\
\hline 40 CFR 266 & Standards lor the Management of Soecific Hazardous Wasles and Specific Tyoes of Hazardous w/as'a Management Facililigs \\
\hline 40 CFR 268 & Land Discoszl Restrictions \\
\hline 40 CFR 270 & EPA Adrinistered permit Programs: The Hazardous Waste Permil Program. \\
\hline $40 \mathrm{CFR} 273$ & Standards for Universal Waste Managemen! \\
\hline 40 CFR 279 & Siandards for the Managemenl of Used Oil \\
\hline 40 CFR 280 & Technical Siandards and Coneclive Aclion Requiements lor Owners and Operalors ol Underorouts Storage Tanks (UST) \\
\hline 40 CFR 300 & Mational Oil and ha zardous Substances qollution Conlingency Plan \\
\hline 40 CFR 302 & Oesignation Reporlable Quanlities, and Notificalion \\
\hline 40 CFR 355 & Emergency Planning and Notificalion \\
\hline 40 CFR 370 & Hazardous Chemical Reporting: Community Right-10 Know \\
\hline 0 CFR 372 & Toxic Chemical Release Reoorting: Communily Righi-to-Know \\
\hline 40 CFA 52 & Approval and Promutation of implementation Plans \\
\hline
\end{tabular}


Table 1.4.2-2 Other Drivers (Continued)

\begin{tabular}{|c|c|}
\hline Name & Title \\
\hline 40 CFB 60 & Standards ol Periormance for New Stalionary Sources \\
\hline 40 CFR 61 & Nalional Emissions Slandards lor Hazardous Air Pollutants \\
\hline 40 CFR 66 & Assessment and Colleclion of Noncompliance Penalties by EPA \\
\hline 40 CFR 68 & Chemica! Acciden! Prevention Provisions \\
\hline 40 CFR 70 & State Operating Pemit Progiams \\
\hline LOCFR 761 & Polychiorinaled Biphenv/s (PCBs) Manulacturing. Processing, Distribution in Commerce. end Use Prohibitions \\
\hline LOCFR82 & Proteclion of Stratospheric Ozone \\
\hline 42 USC 10101 , el sea. & Nuclear Wasle Policy ACl \\
\hline 42 USC 13106 & Source reduction and recycling data coliection \\
\hline 42 USC 2011 , el seq. & . \\
\hline 42 USC $4321 \mathrm{el} \mathrm{seg}$ & The Public Healith and Welfare: Congressional Declaration of Purpose \\
\hline 42 USC 6901, el seq. & Resource Conservation and Recovery Act of 1976 (RCRA). \\
\hline 42 USC 6924 & Standards Applicable to Owners and Operalors of Hazardous Wasle Treatment, Sloraga, and Otsposal Facilities \\
\hline 42 USC 7401 el seo & The Public Kealth and Weliare; Congressional Findings and Declaration of Puroose \\
\hline 42 USC 7671 & Clean Air Act \\
\hline 42 USC 9601 el seg & The Public Health and Weliare: Hazardous Substances Releases, Liability. Compensation Definitions \\
\hline 43 CFA 7 & Prolection of Acchaeological Resources: Unilom Regulations \\
\hline 49 CFP 171 & General Inlomalion, Requlations, and Definitions \\
\hline 49 CFR 172 & $\begin{array}{l}\text { Hazatdous Malerials Table, Special Provisions, Hazardous Malerials Communicalions, Emergency Fezsponse Inlomation, and Training } \\
\text { Regutremenls }\end{array}$ \\
\hline 49 CFR 173 & Shippers, General Requiremenls lor Shipmenls and Packaginas. \\
\hline 49 CFR 175 & Carriage by Airerait \\
\hline 49 CFR 176 & Carriage by Vessel \\
\hline 49 CFR 178 & Specificalions for Packagings \\
\hline 49 CFR 393 & Parts and Accessories Necessary for Sate Opesation \\
\hline 49 CFR 397 & Transportation of Hezardous Malerials: Driking and Parking Rules \\
\hline GCFA571 & Federal Holor Vehicte Sately Standards \\
\hline 49 USC 5101 & Transportation of Hazardous Material \\
\hline 50 CFA 17 & Endariogred and Threatened Wild lite and Plants. \\
\hline 50 CFR 402 & interagency Cooperation - Endangered Species Acl \\
\hline $94 \cdot \mathrm{FDB} \cdot 015$ & Agreement Relating lo Removal and Encapsutation ol Asbeslos Material \\
\hline 95.SFD-191 & Review ol 'DOE Spent Nuclear Fuel Requirements Report (0I: A00000000-00811-1708-0000 Rev, 0)' dated June 6, 1995 \\
\hline $\begin{array}{l}\text { A Reassessment of } \\
\text { Hantord Strategic } \\
\text { Thinking }\end{array}$ & A Reassessment o! Hantord Strategic Thinking, Discussion Materials, Diat, Rev.2, Seplembet 1995 \\
\hline ANS8.7/ANSI.N16.5 & Guide for Nuctear Criticality Sataty in the Slorage of Fissile Malerials \\
\hline ANSI Z88.2.1992 & American National Standard for Resoiralon Prolection, $8 / 6 / 92$ \\
\hline ANSUANS.8.1 & Nuclear Criticality Safery in Operations with Fissionable Materials Outside Reactors \\
\hline ANSI/ANS 8.19 .84 & Asministralive Practices for Nusleat Criticality Salety \\
\hline BCCAA-REGI & Regulation i of the Benton County Clean Air Authority \\
\hline
\end{tabular}


Table 1.4.2-2 Other Drivers (Continued)

\begin{tabular}{|c|c|}
\hline Name & Thile \\
\hline DE.91NM-177 & $\begin{array}{l}\text { Consent Order, In the Matler of the Compliance by USDOE with Chapler } 70.105 \text { and } 90.48 \text { RCW and the Rules and Regulations of the } \\
\text { Department of Ecology }\end{array}$ \\
\hline ONFSB 92.7 & Delense Nuclear Facilities Salety Board Recommendation 92-7, Training and Qualitication \\
\hline DOE $4300.2 C$ & Work for Others (Non-Departmenl of Energy Funded Work) \\
\hline $\begin{array}{l}\text { OOE Document, Plan of } \\
\text { Aclion }\end{array}$ & Pian ol Aclion to Resolve Spent Nucle ar Fuel Vulnerabilities Phase III, Octobet 1994 \\
\hline DOE LeIter 95.SFD-166 & $\begin{array}{l}\text { Choice of Roadway as the Transportation Mode for the U. S. Department of Energy, Fichtand Opsraticns Office K Basin Speni Nuclear } \\
\text { Fuel, Seplember 21, } 1995\end{array}$ \\
\hline DOE Le:ter 95.SFD.218 & U.S. Department of Energyn.S. Department of the Navy/State of Idaho Court Agreemenl \\
\hline DOE LeHer 95-SFD-220 & Approval of Changes to the Operational Salety Requirements (OSA) for the K Basins, December 6,1995 \\
\hline DOE Leller 96-SFO-0DA & $\begin{array}{l}\text { Record of Decision (ROD) for the Final Environmental Impact Statement (FEIS) on the Management cl Spent Nuclear Fuel (SNF) lrom } \\
\text { the K Basins al the Hanford Sile, Richland, Washington, (DOEEIS-0245). March 4, } 1996\end{array}$ \\
\hline DOE M 471.2-1 & Manual for Classified Matter Protection and Control \\
\hline DOE Memo & $\begin{array}{l}\text { Nemorandum ol Agreemenl (MOA) - Utilization ol Canister Storage Building (CSB) Vaults } 2 \text { and } 3 \text { for Immobilized High-level Waste, } \\
\text { April 18, } 1996\end{array}$ \\
\hline DOE Nolice 4700.5 & Projecl Control System Guidelines \\
\hline DOE Nolice 5400.9 & Seaided Radioaclive Source Accountability \\
\hline COE 0130.1 & Budgel Formulation Process \\
\hline DOE 0470.1 & Saleguards and Security Program \\
\hline DOE 0471.2 & Inlomation Security Program \\
\hline DOE 0534.1 & Accounting \\
\hline DOE Ordet 1240.28 & Unclassified and Assignments by Foreign Nationals, B/21/92 \\
\hline DOE Order $1309.2 \mathrm{~A}$ & Departmenl ol Enegay Technisa! Standards Program \\
\hline DOE Order $1324.2 \mathrm{~A}$ & Aacords Disposition, 4/9/92 \\
\hline DOE Order 1324.3 & Files Management \\
\hline DOE Order 1324.5A & Fecords Managemenl Program \\
\hline DOE Ordet 1324.6 & Automaled Otfice Eleckonic Recordkeaping \\
\hline DOE Order 1330.10 & Computer Soltwase Management \\
\hline DOE Otder 1540.1A & Neterials Transoortation and Traffic Manaoement, 1992 \\
\hline QOE Order 1540 ? & Hazzardous Material Packagino for Transport - Administralive Procedures, Change 1, 1988 \\
\hline DOE Order $4330.4 \mathrm{~B}$ & Meinlenance Managemenl Piogram. \\
\hline DOE Order 4700.1 & Project Manzagenenl Syslem \\
\hline $\begin{array}{l}\text { DOE Order } 5000.38 \\
\text { Change } 1\end{array}$ & Occurrence Reporting and Processing ol Operations Information \\
\hline DOE Order 5300.10 & Telesommunications \\
\hline DOE Order 5400.1 & General Environmental Prolection Proosam \\
\hline DOE Order 5400.5 & Rediation Piolection of the Public and the Environment \\
\hline DoE Order 5480.10 & Contracloe Industrial Hygiene Program \\
\hline DOE Order 5480.11 & Radiation Protection for Occupalional Workers \\
\hline DOE Order 5480.19 & Conducl of Operalions Requisements for DOE Facilities \\
\hline DOE Order $5480: 20 \mathrm{~A}$ & Personnel Selection, Qualificalion, Training, and Slatfing al DoE Reactor and Non-Reaclor Nucieat Fasilities \\
\hline
\end{tabular}


Table 1.4.2-2 Other Drivers (Continued)

\begin{tabular}{|c|c|}
\hline Name & Title \\
\hline DOE Ordet 5480.21 & Uneyiewed Salen Quastions \\
\hline DOE Order 5480.22 & Technical Saloly Requirements \\
\hline DOE Orde: 5480.23 & Nuclear Salely Analysis Repon \\
\hline DOE Order 5480.24 & Nuclear Criticality Salety \\
\hline DOE Order 5480.20 & Trending and Analysis of Operations Inlormalion Using Performance indicators \\
\hline DOE Order 5480.28 & Natural Phenomena Hazards Mitigation \\
\hline DOE Order 5480.29 & Employee Concems Management System \\
\hline DOE Order 5480.3 & $\begin{array}{l}\text { Saleh Requirements lor the Packaging and Transportation of Hazardous Materials, Hazardous Subslances, and Hazardous Wasle. } \\
1985 \text {. }\end{array}$ \\
\hline DOE Order 5480.31 & Siatup and Restart ol Nuclear Facilities . \\
\hline DOE Order 5480.4 & Envilongental Proleclion, Salevy and Health Prolection \\
\hline DOE Order $5480.7 \mathrm{~A}$. & Fire Piols slion \\
\hline DOE Ordar $5480.8 \mathrm{~A}$ & Contiacios Occupalionai Medicai Prodram, 6/26/92 \\
\hline DOE Order $5490.9 \mathrm{~A}$ & Censinction Projecl Salely and He alth Managemenl \\
\hline DOE Order 5482.13 & Envionmenl. Satety and Health Aopraisal Program \\
\hline DOE Order 5483.14 & laccupational Saletiand Health Program lor DOE Conlaclor Employees al Govemmeni-Owned Coniractor-Operaled Facilities \\
\hline DOE Order 5484.1 & Environmantal Prolection, Salehy, and Health Proleclion Inlomalion Reporting Requirements \\
\hline DOE Order 5500.18 & Emergency Managament System \\
\hline DOE Order 5500.28 & Emergency Categories, Classes, and Notification and Reporting Requirements, 4/30/8 \\
\hline DOE Order $5500.3 \mathrm{~A}$ & Plenning and Preparedness for Operational Emergencies. \\
\hline DoE Order 5630.113 & SElequaids and Security Proaram, 8/2/94 \\
\hline DOE Order 5630.15 & Saleguards and Security Training Program \\
\hline DOE Order $5631.1 \mathrm{C}$ & Salєguards and Security Awareness Program, 5/4/94 \\
\hline QOE Orde: $5631.2 \mathrm{C}$ & Peisonnal Security Program, 2/17/94 \\
\hline DOE Order $5631.4 \mathrm{~A}$ & Conlrol o! Classified Visils, 7/8/92. \\
\hline OOE Order $5632.1 \mathrm{C}$ & Prolaslion and Control of Salequards and Security Inleres's, $7 / 15 / 94$ \\
\hline COE Order $5633.3 \mathrm{~B}$ & Contiol and Accountability ol Nuclear Malorials, 2/1293 \\
\hline DOE Order 5639.3 & Vigletion of laws, Losses, and incidents of Security Concem, 9 '15/92 \\
\hline DOE Order 5650.23 & Identification ol Classified Intomation \\
\hline DOE Order 5700.20 & Cost Eslimating, Analysis, and Standardization \\
\hline looE Order $5700.6 \mathrm{C}$ & Ougiti Assuiance, 8/21/91 \\
\hline DOE Order $5820.2 \mathrm{~A}$ & Rac oaclive Waste Managemant \\
\hline DoE Order $6430.1 \mathrm{~A}$ & Genaral Dosion Criteria \\
\hline DOE $+15632.3 C \cdot 1$ & WEnval for Prolection and Control of Saleguards and Security Inlerests \\
\hline DOE-PL 5430.1A & Envircrimental, Salety, and Health Program for Fichland Operalions \\
\hline DOE-RL-92-36 & Han'ord Site hoisting and Rigging Menua! \\
\hline DOE.RL-94-02 & Herdord Energency Response Plan \\
\hline DOE.5TD.1020.94 & Nath:al Fhenomena Hazaros Design and Evalualion Criteria for Department of Energy Facilities \\
\hline DOE-STD-1021-93 & Naturei Pnenomena Hazards Perfomance Categorization Guidelines for Structures, Systerns, and Cemponents \\
\hline DOE.STD-1022.94 & Ne!tral Phenomena Hazards Site Characterization Crileria \\
\hline DOE.STD-1023-9j & Na':utel Phenomena Hazards Assessment Criteria \\
\hline
\end{tabular}


Table 1.4.2-2 Other Drivers (Continued)

\begin{tabular}{|c|c|}
\hline Name & Tiille \\
\hline DOE-STO-1073-93 & DOE Standard Guide for Operational Configuration Managernent Program \\
\hline DOEEH.0173T & Environmenlal Requlalory Guide lor Pladiological Effluent Monitoring and Environmental Surveillance \\
\hline DOEEHO135 & Performance Objeclives and Crileria lor Technical Salety Appraisals at Deparment ol Energy Facilitias and Sites \\
\hline DOEEEHO256T(940431) & U.S. Dapartment of Energy Radiological Conlrol Manual \\
\hline DOE/EP.0108 & Standard lor Fire Proleclion of DOE Electronic CompuretDsta Processing Systems \\
\hline DOEEV.0043 & Standat on Fite Proleclion for Portable Structures \\
\hline $\begin{array}{l}\text { DOE Naw/State of } \\
\text { doho Consent } \\
\text { Order/SetilemenlA }\end{array}$ & DOENaw/Siate of Idaho Consent Ordet/Setliement Agreement on Spenl Fusl and Nuclear Waste, October 18, 1995 \\
\hline DOESNFIPP-001 & DOE.Orned Spenil Nuciear Fuel Program Plan \\
\hline $\begin{array}{l}\text { Federal Regisle Nol. } \\
60, \text { No. } 105\end{array}$ & $\begin{array}{l}\text { Programmatic Spent Nuclear Fuel Management and Idaho National Engineering Laboralory Environmental Resloralion and Waste } \\
\text { Managament Programs. Racord ol Decision }\end{array}$ \\
\hline FF-01 & FF-ol Pamil lot Radioaclive Airborne Emission Sources \\
\hline $\begin{array}{l}F F C A \text { lot HESHAP } \\
(2 \pi / 94)\end{array}$ & The National Emission Standards lor Hazardous Air Pollutants: Federal Facility Compliance Agreenrent for the Hanford Site \\
\hline GC-LOAD-01 & Design Loads for Facilities \\
\hline GH-CLIM-01 & Oesign Climale Data lor Hanlord Site \\
\hline $\begin{array}{l}\text { Hantord SNF Siralegic } \\
\text { Plan. }\end{array}$ & Strategic Pian for Managing Spent Nuclear Fuel on the Hantord Site, Draht, Herch 1994 \\
\hline HSRCM-1 & Haniord Sile Radiological Conirol Manual \\
\hline IATA & Inlemalional Air Transport Association, Dangerous Goods Regulations, 1994 \\
\hline $\begin{array}{l}\text { Inlemal Merno, C. } \\
\text { Defigh.Price to L. E. } \\
\text { Ebbeson }\end{array}$ & Slandards/Requirements idenificalion Document Accounlability - Adherence Sell Assessmenl, 2A100-95.007, February 28, 1996 \\
\hline $\begin{array}{l}\text { Inlemal Memo, D. W. } \\
\text { Siddoway to C. L. } \\
\text { Bennet. }\end{array}$ & Memulandum ol Underalanding between K Basins and Analytical Services, February 13, 1996 \\
\hline $\begin{array}{l}\text { Mernorandum of } \\
\text { Agreement }\end{array}$ & Utilizalion ol AMNP Canister Storage Building (CSB) by Spent Nuclear Fuel Project Division (SFD), E-25/95 \\
\hline MRP 1.1 & Managing BOE Directives (from WHC.CM 1.3, Managemen! Requirements and Procsdures) \\
\hline MRP 2.16 & Processing Control Manual Syslem Procedures (from WHC.CM-1-3, Managemen! Requirements and Procedures). \\
\hline MRP 4.16 & Administering Progressive Discipline (from WHC-CM-1-3, Managemenl Requirements and Procedues) \\
\hline MRP 4.19 & Overlime and Shit Differential lor Salaried Non-Exemp! Employees (from WHC.CH: 1-3, Management Requiremen!s and Procedures) \\
\hline MRP 5.44 & Wasta Mininizadion Program (trom WHC-CM-1-3, Managennent Requirenanils and Procedures) \\
\hline MAP 6.15 & Facility Shutdown, Stand By, and Transler (from WHC.CM-1.3, Management Requirements and Procedures) \\
\hline PL 92.500 & Fedatai Wales Pollution Control Act Amendments ol 1972 (Clean Water Acl) \\
\hline PL.100.605 & Hzanoid Re ach Siudy Acl, Comorehensive River Conservalion Sludy \\
\hline RCRA-S(DW)/940829) & Dangatous Wasle Portion of the RCRA Pemil for the Trealmenl, Storage, and Disposal of hazardous W/aste \\
\hline RCW 27.44 & Indian Giaves and Records \\
\hline RCW 27.53 & Archaeological Siles and Resources \\
\hline
\end{tabular}


Table 1.4.2-2 Other Drivers (Continued)

\begin{tabular}{|c|c|}
\hline Name & fitle \\
\hline RCW 46.37 & Vehicle Lighting and Other Equipment \\
\hline RCW 49.17 & Washinglon Industrial Salety and Health Act \\
\hline RCW 70.105 & Washinglon Hazardous Wasle Managemenl Act \\
\hline RCW $70.105 D$ & Model Toxics Conlicl Acl \\
\hline RCW 70.94 & Washinglon Clean Air ACl \\
\hline RCW 70.95 & Solid Waste Managemenl - Fecovery and Recycling \\
\hline RCW 70.98 & Nuclear Energy and Radialion \\
\hline RCW 90.44 & Regula lion ol Public Ground Waters \\
\hline RCW 90.48 & Water Polltution Control Acl \\
\hline ACW 90.52 & Pollution Disclosure Acl ol 1971 \\
\hline RCW 90,76 & Underoround Slorage Tanks \\
\hline PLID $1300.1 C$ & Facility Representative Program \\
\hline RLID 232.1 & Occurrence Reporling and Processing of Operations Informalion \\
\hline RLIO 430.1 & Systems Engineering Criteria Document \& Implementing Diteclive \\
\hline$R L 10471.2$ & Inlomation Security Program \\
\hline RLID 473.1 & Protection of Saleguards and Security Inlerests \\
\hline PLLID 5000.1 & Baseline Execution and Management Process \\
\hline RLID 5000.38 & Occurrence Reporting and Processing of Operations Information \\
\hline RLL1D 5480.31 & Slartup and Restart of Nuclear Facitilies Operalional Readiness Feview and Readiness Assessments \\
\hline RLLID 5480.7 & Fire Prolection \\
\hline BLLID 5630.3A & Protection ol Hanlord Facilities Aoainsl Fiadiological and Toxicological Sabolage \\
\hline RLID 5632.1日 & Assel Prolection Requirements \\
\hline RL10 5533.3 & Control and Accountability of Nuclear Materials at RL \\
\hline F!L $94-04 \hat{3}$ & Hentord Site Stralegic Plan \\
\hline RLIP 5484,1A & Envionmenlal Proleclion, Safely, and Health Prolection Intormation Reporting Requiremenls \\
\hline RLPD 430.1 & Hantord Site Systems Engineering Policy \\
\hline SEN 35-91 & Nuclear Salety Policy \\
\hline $\begin{array}{l}\text { U. S. Govemment } \\
\text { Memo, B.J. } \\
\text { Shackletord lo Dist. }\end{array}$ & Afitachments, Issue Papers on the Applicability of RCRA lo Spent Nuclear Fue! \\
\hline $\begin{array}{l}\text { U.S. Department of } \\
\text { Energy/S.S. } \\
\text { Depatmeni of the N }\end{array}$ & DOE Leller 95-SFD-218. WHC Letter 9504811BA1 \\
\hline WA.000374.3 & Authorization to Discharge Under the National. Pollutant Discharge Elimination Systern \\
\hline WA7890008967.DW & Dangerous Wasle Portion of the RCRA Pemit for the Tieatment, Storage, and Disposal of Hazardous Waste \\
\hline WAC 173.200 & Watat Qualiny Standards lor Ground Walers of the Stale of Washington \\
\hline WAC 173-201A & Waler Qualizy Standards for Surlace Waters of the Siate of Washington \\
\hline WAC 173.216 & Stele Whasie Discharge Permit Program \\
\hline WAC 173.218 & Underground Injeclion Con!rol Program \\
\hline WAC 173.220 & National Pollutant Discharge Elimination Syslem Permil Program \\
\hline WAC 173.221 & Discharge Slandards and Etiluent Limilalions for Domestic Waslevaler Facilities \\
\hline
\end{tabular}


Table 1.4.2-2 Other Drivers (Continued)

\begin{tabular}{|c|c|}
\hline Name & Title \\
\hline WAC 173-225 & Federal Waler Pollution Control Acl-Establishment of Implementalion Procedures ol Application for Certificalion \\
\hline WAC 173-230 & Centification ol Operators of Wostewater Trealment Facilities \\
\hline WAC 173.240 & Submission of Plans and Heports for Consinction of Wastewater Facililies \\
\hline WAC 173.303 & Dangerous Waste Regulalions \\
\hline WAC 173.304 & Minimum Funclional Slandards lor Solid Wasle Handling \\
\hline WAC 173.307 & Plans \\
\hline WAC 173.360 & Underground Storage Tank Regulations \\
\hline WAC $173-400$ & General Requlalions for Air Poilution Sources \\
\hline WAC 173-401 & Ooeraling Pernit Requlations. \\
\hline WAC 173-435.010 & Emergency Episode Plan \\
\hline WAC 173-460 & Conliol for New Sources ol Toxic Air Pollutants \\
\hline WAC 173-470 & Washinglon Air Pollttion Control Regulations, Ambient Air Quality Standard lor Panticulate Malses \\
\hline WAC 173-480 & $\begin{array}{l}\text { Ambient Air Quality Slandards and Emission Limils for Radionuclides } \\
\text { SEPA Piccedures }\end{array}$ \\
\hline WAC 173-802(840615) & SEPA Piocedures \\
\hline WAC 197-11 & SEPA Rules \\
\hline WAC 220.110 & Hydraulic Code Ritles \\
\hline WAC 246.247 & Radiation Prolection - Air Emissions \\
\hline WAC 245-272 & On. Site Sewage System \\
\hline WAC 246.290 & Public Waler Suoplies \\
\hline WAC 246-291 & Group B Public Wa!er Syslems \\
\hline WAC 246.292 & Operalor Ce rification - Water System Operation Renewal of Centificales \\
\hline WAC 246-294 & Drinking Water Operating Permits \\
\hline WAC 296-155 & Salety Stds. lor Construction Work \\
\hline WAC 296.24 & General Salety and Health Standards \\
\hline WAC 296.27 & Recordkeeping and Reporting \\
\hline WAC 296.360 & Biscriminalion, Pursuant to RCW 49.17 .160 \\
\hline WAC 290.65 & Asboslos Removal and Encapsulation \\
\hline WAC $446-50$ & Transponation of Hazardous Materials \\
\hline WAC 463.39 & General and Operating Pemit Regulations for Air Polulion Sources \\
\hline WAC 470.12 & Transporting Rules \\
\hline WAC 51.11 & We shington Stale Energy Code \\
\hline WAR.00-000F & Stomwater Discharge Permit \\
\hline WAR-10.000 F & Stormwaler Discharge Permil \\
\hline YHC Letter 9553985 & Submittal of Conceplual Design Review lo: Canisler Slorage Building, 7/24/95 \\
\hline WHC-CM-1 & Manazement Policies \\
\hline $\mathrm{YHC} \cdot \mathrm{CM}-1 \cdot 10$ & Saley Manual \\
\hline WHC.CM-1.11 & InJustrial Hygiene Marnual \\
\hline WHC.CM. $9 \cdot 5$ & Slandard Operating Praclices \\
\hline $\mathrm{WHC} \cdot \mathrm{CM} \cdot 1-8$ & Work Hanagement \\
\hline WHC.CM-2-10 & Cosi Accounting Manual \\
\hline
\end{tabular}


Table 1.4.2-2 Other Drivers (Continued)

\begin{tabular}{|c|c|}
\hline Name & Thile \\
\hline MHC.CM+2-14 & Hazardous Malerial Packaging and Shipging \\
\hline WrAC.CM-2.15 & Training Adminisistration Manual \\
\hline WHC-CM-2.2 & Materials Managemenl Manual \\
\hline WHC.CM.2.3 & Propenty Hanagament Manual \\
\hline WHC.CM-2.5 & Mlanagemenl Control Syslem \\
\hline WHC.CH.3.10 & Sotwiare Piaclices \\
\hline $\mathrm{W}-\mathrm{HC} \cdot \mathrm{CM} \cdot 3 \cdot 5$ & Dosument Conlsol and Records Management Manual \\
\hline WHC.CH:4.14 & Aqpilied Ratiological Controls \\
\hline WHC.CM-4.16 & Dosimelry and Medical Services Manual \\
\hline$W H C \cdot C M \cdot 4 \cdot 2$ & Qualiny Assurance Manua! \\
\hline WHC.CN-4.29 & Nuclear Criticality Salen Manual \\
\hline WHC.CN.4.33 & Securing Manval \\
\hline WHC.CM-4.40 & Industrial hiviene Manual \\
\hline WHC.CM-4-41 & Fire Proleclion Program Nlanual \\
\hline WHC.CM-4-43 & Emergency Mlanagemenl Procedures \\
\hline WHC.CM-4.46 & Nonceactor Facility Salehr Analysis Manual \\
\hline WHC.CM-4.5O & Saleguards Accounting Manual \\
\hline WhHC-CH-4.7 & Unciassified Compuler Security \\
\hline WHC-CM-5-13 & K Besin Policy Menual \\
\hline WHC.CM-6.1 & Slenderd Engineering Preclices \\
\hline WHC.CH.6.10 & Welding Mlanual \\
\hline WHC-CM.6-2 & Prolecl Manzoement \\
\hline WHC-CM-6-3 & Drating Standards Manuai \\
\hline WHC-CM-7.5 & Envisonmental Compliance \\
\hline WHC-CM-8.7 & Operalions Support Services \\
\hline WHC-EP-0063.4 & Hanlord Site Solid Wasle Acceptence Criteria \\
\hline WhiC.EP-0495 & WhC Pollution Prevention Program Implementation Plan \\
\hline WHC.EP.0790 & Sp=nt Huclear Fusil Project Wission Analysis Repor \\
\hline WHC-EP-OB30 & Hantord Spent Nuclea: Fuel Project Fecommended Path Forward \\
\hline WHC-EP.0853 & DNFSB Recommendation 94-1 Hanlord Sile Inlegrated Slabilizalion Management Plan \\
\hline WHC.IP-0565 & Saleguards Dosk Procedures \\
\hline WHC-IP-0702 & Who ico Aiea Emergency Response \\
\hline WHC.IP-0821.PUO & Plutonium Opzation Administration \\
\hline$W H C \cdot \mid P \cdot 1043$ & WhL Occupational ALARA Program \\
\hline$W / H C \cdot P \cdot 1140$ & Technical Frocedure Development and Conitrol \\
\hline WHC-SD-GN-DGS.3001 & $\begin{array}{l}\text { Redialogicel Design Guide } \\
\end{array}$ \\
\hline W/HC-SO-GN.ER-10OOE & KBasin: Floor Loads and Calculation \\
\hline WHC-SO-GN-ES-30006 & Sitz Dasign Crileria lor Uniform Bolting Preloads \\
\hline
\end{tabular}


Table 1.4.2-2 Other Drivers (Continued)

\begin{tabular}{|c|c|}
\hline Name. & Title \\
\hline $\begin{array}{l}\text { WHC.SD-GN-ICO-001, } \\
\text { Rev. } 1\end{array}$ & Inlerlace Agreement for the Managemenl of the 308 Building Spent Nuclear Fuel, December 22,1995 \\
\hline WHC.SO-NR.SA.024 & 105-KE/105-KW Irradialed Fuel Storage Basins Seismic Qualification \\
\hline WHC.SD-SNF.CM-001 & Spent Nuclear Fuel Projecl Conliguralion Management Plan \\
\hline WHC.SD-SNF.CM-0O3 & Spent Nuclear Fuel Project Interface Control Plan \\
\hline $\begin{array}{l}\text { WHC.SD-SNF-OB.002, } \\
\text { Aev, } 1\end{array}$ & Spenl Nuclear Fuel Project Path Forward - Nuclear Salely Equivelency lo Comparable NRC-Licens:d Facilities; dated June 3, 1996 \\
\hline WHC:SD-SNF-DQ-003 & Spent Nucloar Fuel Projecl Path Forward Additionai NAC Requitements \\
\hline WHC.SD-SNF.08.004 & Spent Nuclear Fuel Project Seismic Design Criteria \\
\hline WHC.SD.SNF.0B-005 & U.S. NRC Salely Equivalency Requiremenis; Multi-Canisler Overpack Additional NRC Ręquisenast's \\
\hline WHC-SD-SNF-DE-009 & CS9 Na!urat Phenomena Hazards LA015, Revision 1, June 10, 1996 \\
\hline WHC-SD-SNF.DB-010 & Cold Vacuum Drying Syslem Natural Phenomena Hazards, Revision 0, June 7, 1995. \\
\hline WHC-SD-SNF-DGS.001 & $100 \mathrm{~K}$ Basins Design Guidelines \\
\hline WHC-SD.SNF.DGS.002 & SNF Project, Equipment and Piping Labeling Guide \\
\hline $\begin{array}{l}\text { WHC-SD-SNF.HC-011. } \\
\text { ReV.0 }\end{array}$ & Hazard Calegorization ol K Bas in Fual Characierization, Phase Il, February 7, 1996 \\
\hline WHC-SO-SNF.OCD.0O & Spenl Nucle ar Fuel Condilioning Producl Crileria, February 23, 1996 \\
\hline WHC-SD.SNF.PLN-011 & Speni Nuctear Fuel Project Nuclear Safely Regulatory Program Plen \\
\hline WHC-SD-SNF-PMP-01 & Spent Nuclear Fuel Project, Projecl Management Plan \\
\hline WHC.SO-SNF-OAPP.OD & $\int_{4}$ SNF Project, Quality Assurance Program Pian \\
\hline WHC-SD.SNF-RPT+C07 & $\begin{array}{l}\text { Application of the Otfice of Civilian Radioactive Wasle Management Ovality Assurance Requiraments to the Mantord Spent Nuclear } \\
\text { Fuel Project }\end{array}$ \\
\hline WHC.SD.SNF.SEMP.OS & Spent Nuclaar Fual Project Systsms Engineering Kanagemenl Pien \\
\hline WHC.SD-SNF-SP-OO2 & Spen! Nuclear Fuel Regulalory Strategy \\
\hline WHC.SO-SNF.SP.007 & SNF Projed, SNF Accounlability Plan \\
\hline $\begin{array}{l}\text { WHC.SD-SNF-SP.009, } \\
\text { Rev. } 0\end{array}$ & $105 \mathrm{~K}$ East and $105 \mathrm{~K}$ West Fuel Transfer Bay Crane Use Siralegy lor Spant Kuclear Fül Path Formard \\
\hline WHC-SD.SNF-SP-010 & SNF Project, Dose Managemenl Plan \\
\hline WHC-SD-SNF-TI.009 & $105 \mathrm{~K}$ Básin Material Design Ba sis Feed Description for Spenl Nuclear Fuel Project Fazilitis \\
\hline WHC-SD-SNF-TI-O16 & Developmenl of Design Basis Capacity for SNF Projecl Syslems \\
\hline WHC.SO-WMAOSR.006 & Operalions Sa'ely Requirements - $100 \mathrm{~K}$ Easi and $100 \mathrm{~K}$ West fuel Storage Basins \\
\hline WAC.SOWWASAR.0S2 & Sately Analysis of liradialed N. Peactor Fuel \\
\hline WHC.5P.0708 & Wes linghouse Hentord Compazy Conducl of Operalions Manual \\
\hline WHC.SP.C366 & Westinghouse Conducl of Maintenance Manual \\
\hline C.SP.1104 Re' I. & Spen! Nuclear Fuel Projecl 1995 Mulli-Year Program Pla \\
\hline
\end{tabular}


Table 1.4.2-2 Other Drivers (Continued)

\begin{tabular}{|l|l|l|}
\hline Name & Title \\
\hline WHC-SP-1131 & Weslinghouse Hanlord Company OA Piogram and implementalion Plan \\
\hline
\end{tabular}

\subsection{FUNCTIONAL DEFINITIONS}

This section contains the Hanford Site Technical Baseline functions that are assigned to the project. If describes the project work in terms consislent with the Hanford Site Technical Baseline. The 'number' column in this section refers to the unique function number for the listed function as contained in the site technical baseline database maintained by Site Systems Engineering.

\subsubsection{Manage SNF Disposition System}

Table 1.5.1-1 Manage SNF Disposition System Functions

\begin{tabular}{|c|c|}
\hline Number & Name / Osscriotion \\
\hline 1.0 & 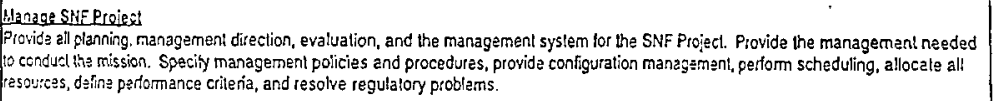 \\
\hline 3.0 & 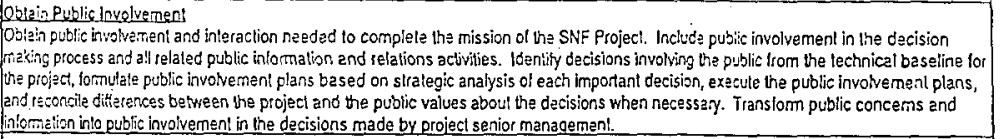 \\
\hline 3.1 & 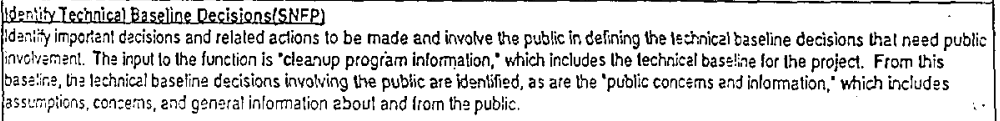 \\
\hline 3.2 & 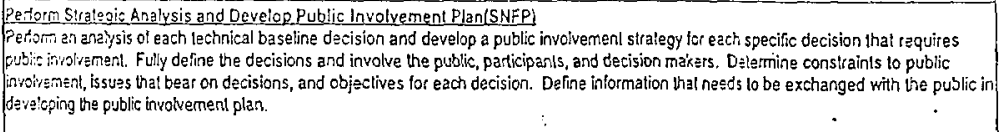 \\
\hline 3.3 & 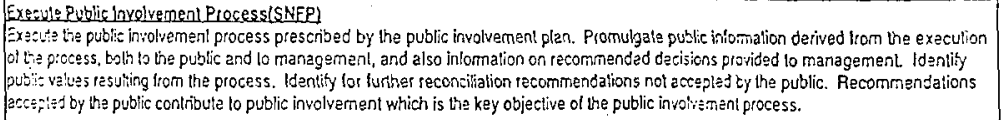 \\
\hline 34 & 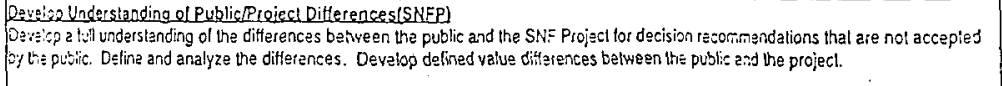 \\
\hline 3.5 & 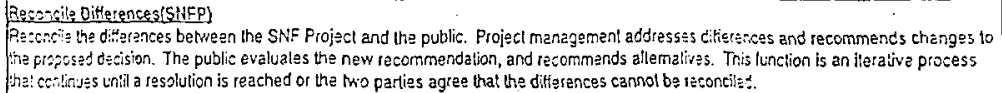 \\
\hline
\end{tabular}

\subsubsection{Acquire SNF Disposition System}


Table 1.5.2-1 Acquire SNF Disposition System Functions

\begin{tabular}{|c|c|}
\hline Number & Name / Descriplion \\
\hline 2.0 & $\begin{array}{l}\text { Aceuire SUE Projact Missien Essential Capabilities } \\
\text { Provida all new intellectual and physical resources. This includes personnel, consultanls, services, supplies, equipment, struclure s, systems. } \\
\text { and components that are supplied by the site contractors, construction projects, and subcontracts ol all kinds. }\end{array}$ \\
\hline 2.1 & 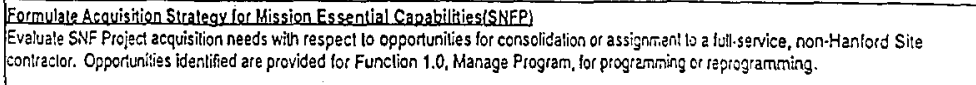 \\
\hline 2.2 & $\begin{array}{l}\text { Provide Expertise(SNFP) } \\
\text { Provide new parsonnel and relrain existing professional personnel for new assignments oulside their usual discipline, provide expent } \\
\text { consullants, and a cquire ofhsite expert services ol all types. }\end{array}$ \\
\hline 2.3 & 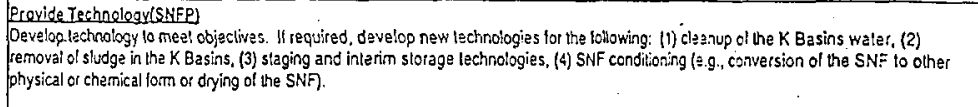 \\
\hline 2.4 & $\begin{array}{l}\text { Provide Facilities, Equioment Inlrastructure and Supolies(SNFP) } \\
\text { Produce all lacilities, equipmen!, soltware, and related ilems needed loc all work aclivities, including ail systems, subsyslems, componenis, } \\
\text { and structures. }\end{array}$ \\
\hline 2.5 & 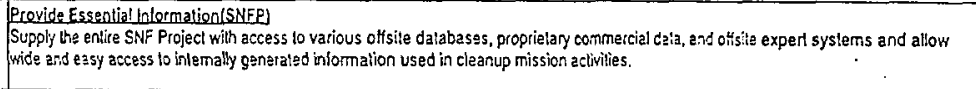 \\
\hline 2.6 & $\begin{array}{l}\text { Provide Integrated independenl Services(SNFF) } \\
\text { Delive: integraled service conliacls tor other inlegration ettorts. }\end{array}$ \\
\hline
\end{tabular}

\subsubsection{Operate \& Maintain SNF Disposition System}

Table 1.5.3-1 Operate \& Maintain SNF Disposition System Functions

\begin{tabular}{|c|c|}
\hline Number & Name / Descriplion \\
\hline 4.7 .2 & 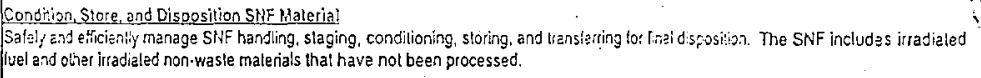 \\
\hline 4.7 .2 .1 & 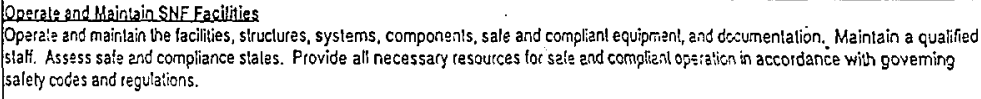 \\
\hline 4.7 .2 .2 & 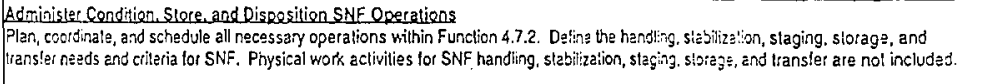 \\
\hline 4.7 .2 .3 & $\begin{array}{l}\text { Stage K Easins SMF } \\
\text { Receive shipments of SWF trom K Basins, provide shortterm slorage of the SNF, and stage the SNF for tienster lo conditioning. }\end{array}$ \\
\hline 4.7 .2 .4 & 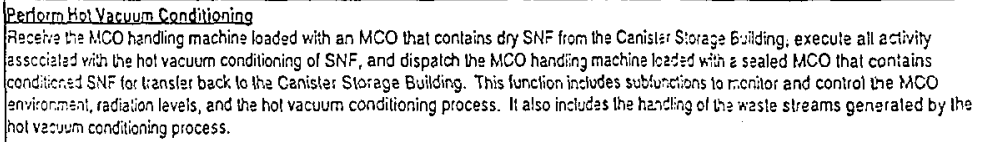 \\
\hline
\end{tabular}


Table 1.5.3-1 Operate \& Maintain SNF Disposition Syslem Funclions (Continued)

\begin{tabular}{|c|c|}
\hline Number & Name / Descriplion \\
\hline 4.7 .2 .5 & $\begin{array}{l}\text { Store SNF } \\
\text { Provide inlerim storage of conditioned K Basins SNF at the Canisler Storage Building. Also provide inlerim slorage of other SNF al the } 200 \\
\text { Area and } 400 \text { Rrea interim slorage areas. }\end{array}$ \\
\hline 4.7 .2 .6 & $\begin{array}{l}\text { Stage SNF for Final Disposition } \\
\text { Prepare, condition, and iransler all Hanlord Site SNF for final disposition. Disposition the SNF by one of trio processes: Iranslat usabie SNF } \\
\text { or transler materials lor disposal. Anange for disposal ol incidental wastes during disposition process. }\end{array}$ \\
\hline
\end{tabular}

\subsubsection{Dispose of SNF Disposition System}

Table 1.5.4-1 Dispose of SNF Disposition System Functions

\begin{tabular}{|c|c|}
\hline Number & Name / Descriplion \\
\hline 4.1 .1 .9 & $\begin{array}{l}\text { Deactivate SNF (Other) } 400 \text { Area } \\
\text { Deaclivate the SNF (Other) } 400 \text { Araa including the transler of the SNF (Other) to the SNF (Other) } 200 \text { Area. This includes the lacility and } \\
\text { equipment. }\end{array}$ \\
\hline 4.1 .1 .10 & $\begin{array}{l}\text { Deactivale Hol Vacuum Conditioning System } \\
\text { Deaclivate the Hot Vacuum Conditioning Facitity. This includes lacility intrastruclure and associaled equipment. }\end{array}$ \\
\hline 4.1 .1 .11 & $\begin{array}{l}\text { Deactivate Canister Storase Building (CSB) } \\
\text { Deactivate the Canister Storage Buitding. This includes facilities, associaled equipment, and infrastructure, as well as the transtar of the SNF }\end{array}$ \\
\hline 4.1 .1 .12 & $\begin{array}{l}\text { Deactivate SHF (Other) } 200 \text { Area } \\
\text { Deaclivate the SNF (Other) } 200 \text { Area. This includes the transler of the tuel as well as the tacility, associatis equipment, and intraslructure. }\end{array}$ \\
\hline 4.1 .1 .14 & $\begin{array}{l}\text { Deactivate Cold Vaeuum Drying System } \\
\text { De activale the cold vacuum dying systen. This includes system inlrastructure and associated equipment. }\end{array}$ \\
\hline
\end{tabular}

\subsubsection{Manage K Basin Deactivation System}

\section{Table 1.5.5-1 Manage K Basin Deactivation System Functions}

\begin{tabular}{|c|c|}
\hline Number & Name / Oescription \\
\hline 1.0 & $\begin{array}{l}\text { Hanage SH.JF Project } \\
\text { Provide ell plenning, management direction, evaluation, and the managemenl system for the SNF Project. Frovide the management needed } \\
\text { to conduct the mission. Specity managemenl policies and procedures, provide configuration managemenl, partorm scheduling, allocate all } \\
\text { resoutces, detine pertomance criteria, and resolve regulatory problems. }\end{array}$ \\
\hline 3.0 & 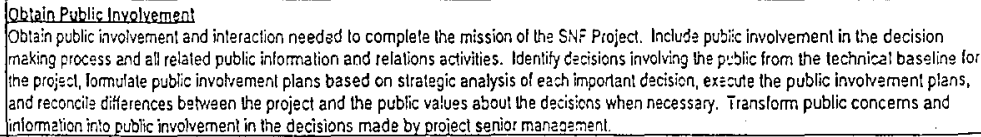 \\
\hline 3.1 & 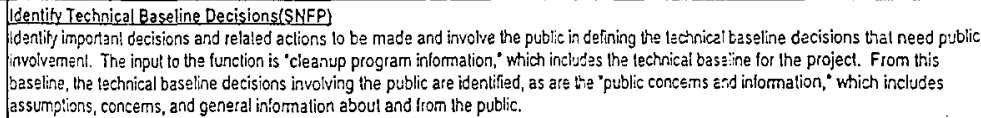 \\
\hline
\end{tabular}


Table 1.5.5-1 Manage K Basin Deactivation System Functions (Continued)

\begin{tabular}{|c|c|}
\hline Number & Name / Description \\
\hline 3.2 & 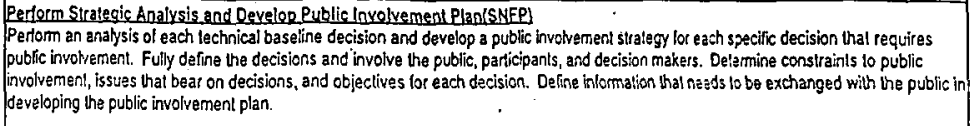 \\
\hline 3.3 & 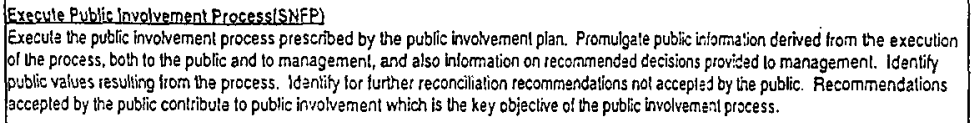 \\
\hline 3.4 & 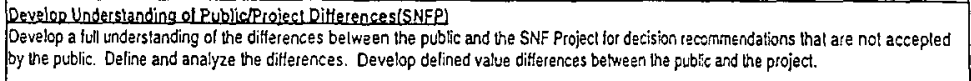 \\
\hline 3.5 & $\begin{array}{l}\text { Aeconcile Difterences:SNFP) } \\
\text { Aeconcile the difterences beiween the SNF Project and the public. Project managemenl addresses ditterences and recommends changes to } \\
\text { the proposef decision. The pubtic evaluates the new recommendation, and recommends altematives. This tunction is an iterative process } \\
\text { thal continues until a tesolution is reached or the two parties agree that the difterences cannol be teconciled. }\end{array}$ \\
\hline
\end{tabular}

\subsubsection{Acquire K Basin Deactivation System}

Table 1.5.6-1 Acquire K Basin Deactivation System Functions

\begin{tabular}{|c|c|}
\hline Number & Name/Descriplion \\
\hline 2.0 & $\begin{array}{l}\text { Acquire SNF Project Mission Essential Capabilities } \\
\text { Provide all new intellectual and physical resources. This inciudes personnel, consultants, sevicas, supplies, equipment, stuclures, syslems, } \\
\text { and components thal are supplied by the site contractors, cons!nction projects, end subcontracls ol all kinds. }\end{array}$ \\
\hline 2.1 & $\begin{array}{l}\text { Eormulate Aequisition Strategy for Mission Essential Capabilitias(SNFP) } \\
\text { Evaluate SNF Project acquisition needs with respect to opportunities lor consolidalion or assignment to a full-service, non-Hanlord Sile } \\
\text { conlraclor. Opportunities identified are provided tor Function } 1.0, \text { Manage Program, lor programming or reprogramming. }\end{array}$ \\
\hline 2.2 & 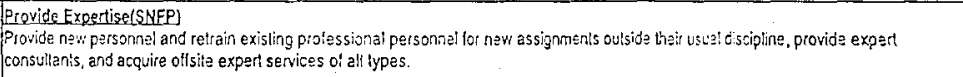 \\
\hline 2.3 & $\begin{array}{l}\text { Provide Technelogy(SNFP) } \\
\text { Develop lechnology lo meel objectives. If required, devalop new technologies tor the following: (1) cleanup of the K Basins water, (2) } \\
\text { removal of sludge in the K Basins, (3) slaging and interim storage technologies, (4) SNF conditioning (e.9. Conversion of the SNF to other } \\
\text { physical or chemical lom or drying of the SNF). }\end{array}$ \\
\hline 2.4 & 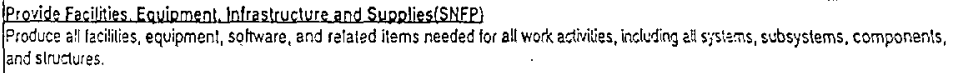 \\
\hline 2.5 & $\begin{array}{l}\text { Provide Essential Inlormation(SHFP) } \\
\text { Supply the entire SNF Project with access to various oflsite databases, proprietary commercial data, end ohsite expert sys tems and allow } \\
\text { wide and ezsy access to intemally generated intorination used in cleanup mission activities. }\end{array}$ \\
\hline 2.6 & $\begin{array}{l}\text { Provide Integrated Independent Services(SNFP) } \\
\text { Defiver integra!ed service contracts for other iniegration eftorts. }\end{array}$ \\
\hline
\end{tabular}

\subsubsection{Operate \& Maintain K Basin Deactivation System}


Table 1.5.7-1 Operate \& Maintain K Basin Deactivation Syslem Functions

\begin{tabular}{|c|c|}
\hline Number & Name/Descriotion \\
\hline 4.1 .1 .8 & $\begin{array}{l}\text { Deactivate K Basins } \\
\text { Deactivale K Basins. Remove SNF, sludge, debris, contaminaled water, and other radioactive or hazardous malerials. Include K Basins } \\
\text { facilities and equipment. }\end{array}$ \\
\hline 4.1 .1 .8 .1 & $\begin{array}{l}\text { Operate and Maintain K Basins During Deactivation } \\
\text { Operate and mainain the K Basins facilities, systems, and equipment. Maintain a qualifed stat, sate and compliant equiprnent, and } \\
\text { documentation. Provide assessment of saley condritons and slate ol compliance. Provide all necessary resources for sate and compliant } \\
\text { operation in accordance with goveming salety codes and regulations according to the K Basin standards'requitements identification } \\
\text { document. }\end{array}$ \\
\hline 4.1 .1 .8 .2 & 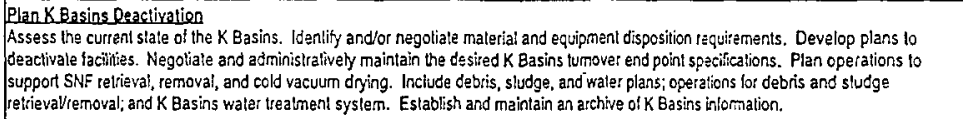 \\
\hline 4.1 .1 .8 .3 & $\begin{array}{l}\text { Disposition K Basins Materials } \\
\text { Execule activities necessary to pertorm and suppon SNF removal while mainlaining accounilabitity of SNF at all times. This includes } \\
\text { characterization and categonzation ol materials and sludge, wate:, and debris supporting aclivilies. }\end{array}$ \\
\hline 4.1 .1 .8 .4 & $\begin{array}{l}\text { Perform K Basins Deactivation } \\
\text { Deactivate nonessential syslems, syslem components, and physical structures. Take other actions, as required, lo minimize environmental, } \\
\text { public, and personnel hazards; ensure actions laken are consistent with minimizing continuing costs a! the K Basins. }\end{array}$ \\
\hline
\end{tabular}

\subsubsection{Dispose of K Basin Deactivation System}

Table 1.5.8-1 Dispose of K Basin Deactivation System Functions

\begin{tabular}{|l|l|}
\hline Number & Name / Description \\
\hline 4.1 .1 .8 .4 & Petorm K Basins Deactivation \\
& $\begin{array}{l}\text { Deaclivate nonessential systems, system components, and physical struclures. Take other actions, as requifed, lo minimize envisonmental. } \\
\text { public, and personnel hazards; ensure actions taken are consistenl with minimizing conlinuing cos's al tha K Basins. }\end{array}$ \\
\hline
\end{tabular}

\subsection{PROJECT LIFE-CYCLE REQUIREMENTS}

This section contains the requirements for each project life cycle phase including the project management requirements.

\subsubsection{Manage SNF Disposition System}

\subsubsection{Manage SNF Project}

\subsubsection{Obtain Public Involvement}

\subsubsection{Acquire SNF Disposition System}

1.6.2.1 Acquire SNF Project Mission Essential Capabilities 


\begin{tabular}{|c|c|}
\hline 1 & $\begin{array}{l}\text { SNEP(103.056) } \\
\text { Requirement Relerence: SNF-RD-PM-00 Rev 0, Section } 4.1 .1 \\
\text { Systems Approach. A stnucluzed, risk-based management approach is required to inlegrale, coordinale, and optiriza the activilies necessary lor } \\
\text { conditioning, handing, and transportation of SNF in the near lerm, for interim slorage, and in preparing the SNF for permanent disposal. }\end{array}$ \\
\hline 2 & $\begin{array}{l}\text { SNFP(103067 } \\
\text { Fequirement Pelerence: SNF.AD.PM.001 Rev 0, Section } 4.1 .1 \\
\text { Systems Approach. A systems approach shall be used to support program integration and to provide a strictured, Icgical, documenlable, and } \\
\text { delensible approach to planning. }\end{array}$ \\
\hline
\end{tabular}

\subsubsection{Operate \& Maintain SNF Disposition System}

Table 1.6.3-1 Operate \& Maintain SNF Disposition System Requirements

\begin{tabular}{|c|c|}
\hline 1 & $\begin{array}{l}\text { MOD. Reacters on the River, Salety Obiective } 538 \\
\text { Provide survell ance and mainlenance of the } 1705 \text { KEKKEUKER Facility. }\end{array}$ \\
\hline 2 & 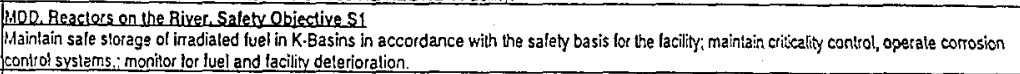 \\
\hline 3 & $\begin{array}{l}\text { HOD Reacters on the River. Final, 1a } \\
\text { Construet Canister Storage Building }(\mathrm{CSB}) \text {. }\end{array}$ \\
\hline 4 & $\begin{array}{l}\text { Moo. Reaclors on the Rixer. Final, ib } \\
\text { Consliucl Co!d Vacuum Drying Facility. }\end{array}$ \\
\hline 5 & $\begin{array}{l}\text { HDQ Reaciors on the River. Final 1c } \\
\text { insiall Hol Vacuum Drying Facitity }\end{array}$ \\
\hline 6 & $\begin{array}{l}\text { 1900. Reacjors on the River. Final 1d } \\
\text { Uporade K. Basin Facilty }\end{array}$ \\
\hline 7 & $\begin{array}{l}\text { Mpo. Reactiors on the River. Final. ie } \\
\text { Insiall fuel relrieval system. }\end{array}$ \\
\hline 8 & $\begin{array}{l}\text { MOD. Reacjors on the River. Final. } 11 \\
\text { Fabricate transpontalion syslem and casks. }\end{array}$ \\
\hline 9 & $\begin{array}{l}\text { MOD, Reaclors on the River, Final, ig } \\
\text { Fabricale multi-canister overpack containers, }\end{array}$ \\
\hline 10 & $\begin{array}{l}\text { HoD. Aeactors on the River, Final, } 1 \mathrm{~h} \\
\text { Ac move sludge } 0 \text { lank farms in the } 200 \text { Area for storage and treatment. }\end{array}$ \\
\hline 11 & $\begin{array}{l}\text { MOQ. Reastors on the Rivec. Final. I1 } \\
\text { rabricale siudae removal system. }\end{array}$ \\
\hline 12 & $\begin{array}{l}\text { MQN. Reacters on the River Final. } 11 \\
\text { Remove debris for decontamination and disposal. }\end{array}$ \\
\hline 13 & $\begin{array}{l}\text { MOD, Reactors on the River, Final } 1 \mathrm{k} \\
\text { Procure debris decontamination services. }\end{array}$ \\
\hline 14 & $\begin{array}{l}\text { HoD Reactors on the River Final. 11 } \\
\text { Peduce radionuclides (except trilium) using basin water puriticalion syslem. }\end{array}$ \\
\hline 15 & $\begin{array}{l}\text { Hop Reactors on the Hiver. Final, im } \\
\text { Uggrade besins watar pyrification syslem. }\end{array}$ \\
\hline 16 & 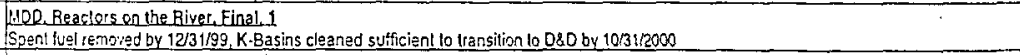 \\
\hline 17 & $\begin{array}{l}\text { Pisp Central Flateau, Interim } 3 a \\
\text { Permils lor canister slorgao bidd obtained. }\end{array}$ \\
\hline 18 & $\begin{array}{l}\text { HoQ Central Plateau, Interim. 3b } \\
\text { FFTF maleriats placed in inlerim slorage. }\end{array}$ \\
\hline 19 & 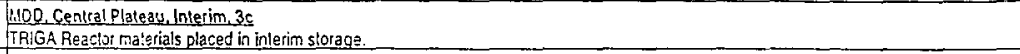 \\
\hline 20 & $\begin{array}{l}\text { HOQ Centrat Platealu Interim 3d } \\
\text { SWR PWB olaced in interim slorage. }\end{array}$ \\
\hline 21 & $\begin{array}{l}\text { WDO, Central Plateau, Interim. 3e } \\
\text { B.Cell Cleanoul Special Case Fuel placed in interim slorage. }\end{array}$ \\
\hline
\end{tabular}


Table 1.6.3-1 Operate \& Mainlain SNF Disposition Syslem Requiremenls (Continued)

\begin{tabular}{|c|c|}
\hline 22 & 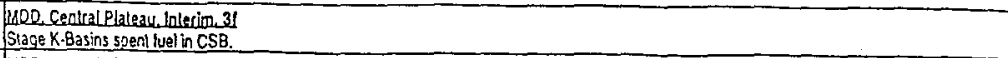 \\
\hline 23 & 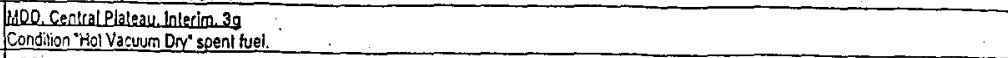 \\
\hline 24 & $\begin{array}{l}\text { HDO Central Plategu, Interim. } 3 \mathrm{~h} \\
\text { Siore corditienes fuel is CSB. }\end{array}$ \\
\hline 25 & $\begin{array}{l}\text { WDQ Central Plateau, Inlerim. } 3 \mathrm{i} \\
\text { Obtain National Reoository waste and packaging criteria (WAC \& PAC). }\end{array}$ \\
\hline 25 & $\begin{array}{l}\text { HDD Central Plateau, Final } 39 a \\
\text { Assure sognal lua's are sale. slable, and 'road-ready. }\end{array}$ \\
\hline 27 & $\begin{array}{l}\text { HoD, Central Plaleau Final } 39 \mathrm{~b} \\
\text { Provide inserim slofage. }\end{array}$ \\
\hline 28 & 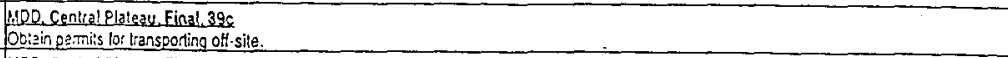 \\
\hline 29 & $\begin{array}{l}\text { HQD Cenlral Plateau Final } 398 \\
\text { Oblain Nzational Repository wasle and packaging criteria IWAC \& PACl. }\end{array}$ \\
\hline 30 & 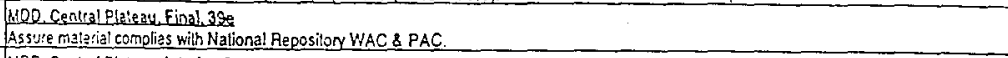 \\
\hline 31 & 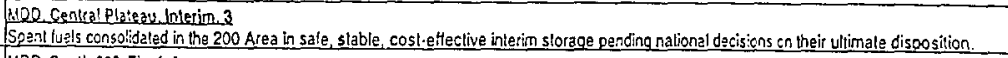 \\
\hline 32 & 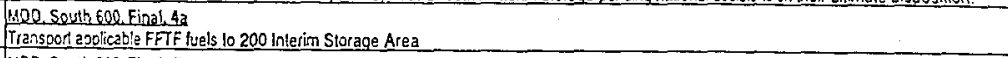 \\
\hline 33 & WOR South 600 Final $4 \mathrm{~b}$ \\
\hline 34 & $\begin{array}{l}\text { HOD. Squth } 600 \text { Final } 4 \mathrm{c} \\
\text { Iranspor lishi vialer reaclor fueis } 10200 \text { isA. }\end{array}$ \\
\hline 35 & $\begin{array}{l}\text { MOQ South } 600 \text { Final } 40 \mathrm{a} \\
\text { Assure malerials are salo, stablg, road-teady: }\end{array}$ \\
\hline 35 & 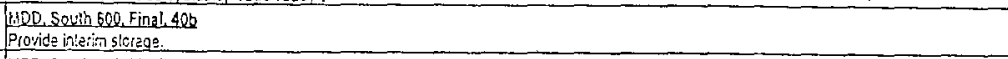 \\
\hline 37 & 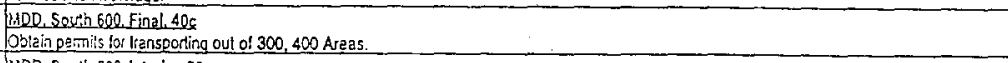 \\
\hline 38 & 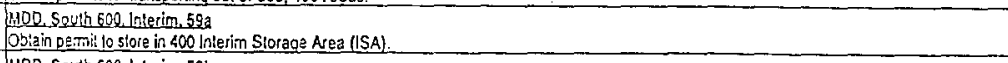 \\
\hline 39 & THOQ South 600 . Interim 596 \\
\hline 40 & 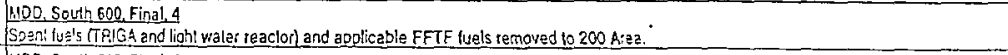 \\
\hline 31 & 190, Swuth E2R, Final, 40 . \\
\hline 12 & 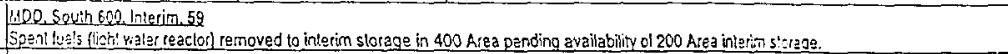 \\
\hline 43 & 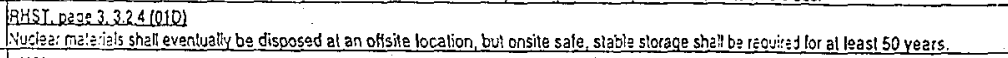 \\
\hline 4 & 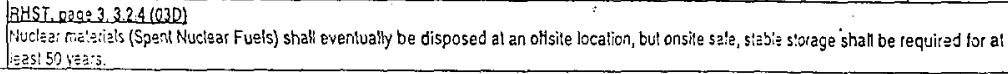 \\
\hline
\end{tabular}

\subsubsection{Condition, Store, and Disposition SNF Material}




\begin{tabular}{|c|c|}
\hline 1 & 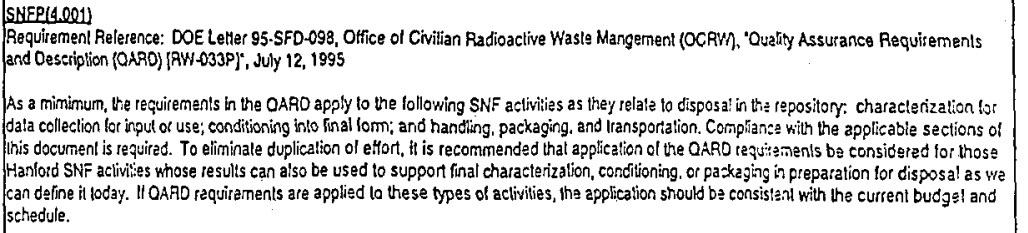 \\
\hline 2 & 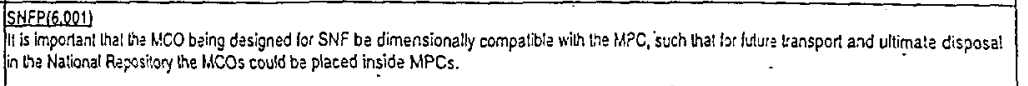 \\
\hline 3 & $\begin{array}{l}\text { SNEP(9.00) } \\
\text { The existing CSB site and loundation will be liansiarred from the Tanix Waste Fernedia'ion System (TWF) to SFD. Secondary priorities witl be lo } \\
\text { utilize excess CSB spaca lo store other Hanlord spent luels or radioaclive malerials. }\end{array}$ \\
\hline 4 & 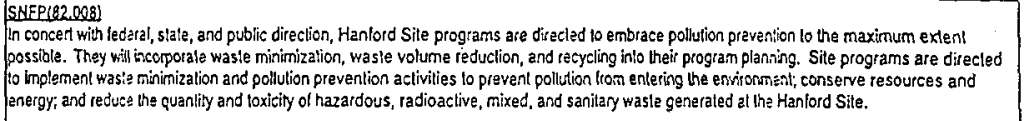 \\
\hline 5 & 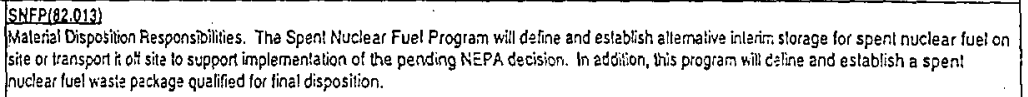 \\
\hline 6 & 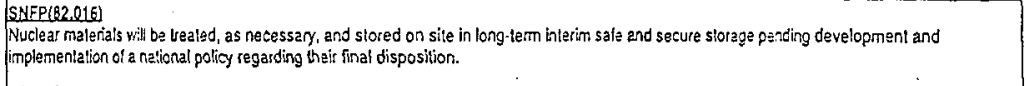 \\
\hline 7 & $\begin{array}{l}\text { SNFP(82.022) } \\
\text { Paragraph 19. Store SNF destined tor ofi-site disposition al an on-site location uniti off site locations are prapared to accept SNF. }\end{array}$ \\
\hline 8 & PNAFP(82.023) \\
\hline 3 & 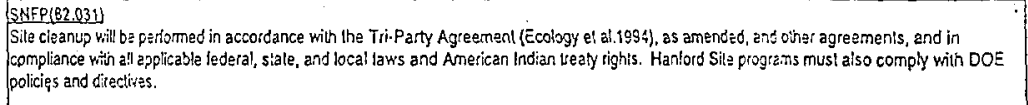 \\
\hline 10 & 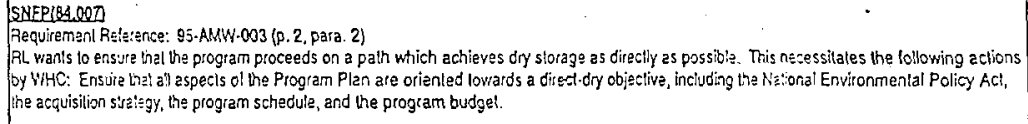 \\
\hline 11 & 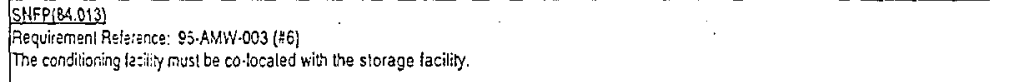 \\
\hline 12 & 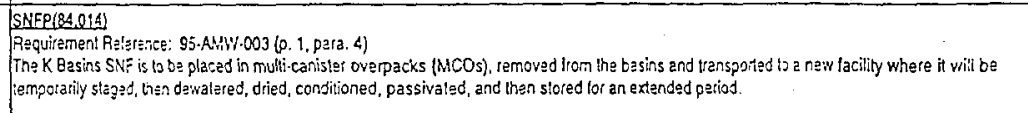 \\
\hline 13 & 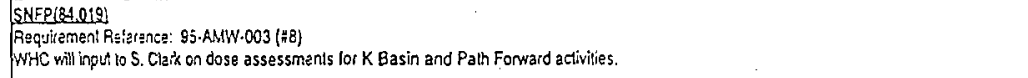 \\
\hline
\end{tabular}




\section{Table 1.6.3.1-1 Condition, Store, and Disposition SNF Material Requirements (Contunued)}

\begin{tabular}{|c|c|}
\hline 14 & $\begin{array}{l}\text { SNEP(B5,001) } \\
\text { Along with the very aggressive ALARA dose reduction ethort required for the fuel reracking process, a project-kide dose managemenl etfort, } \\
\text { Including a folal project dose budget, is needed. }\end{array}$ \\
\hline 15 & $\begin{array}{l}\text { SNEP(B5.005) } \\
\text { WHC is directed to proceed and implement the integraled process strategy. }\end{array}$ \\
\hline 16 & $\begin{array}{l}\text { SNEP(103.001) } \\
\text { Requirement Relerence: SNF.RD-PM-001 Rev 0, Section } 4.2 .5 \\
\text { Accident Radioactive Feleases. New SNF facilities shall be designed, consinucted, and cperated such that a tar a design basis accidant, potential } \\
\text { exposure lo radiation shall be within regulatory requirements, as specilied in applicable sections of Title } 10 \text { of the Code of Federal Regulations } \\
\text { (CFR). }\end{array}$ \\
\hline 17 & $\begin{array}{l}\text { SNFP(103,003) } \\
\text { Fequirement Relarence: SNF-RD-PM-001 Rev 0, Section } 5.3 .8 \\
\text { Assurance of Storage Viability. Criteria and methods, appropriate to each fuel form and storage method, shall be eslablished fo assure continued } \\
\text { sale slorage of DOE-owned SNF. }\end{array}$ \\
\hline 18 & $\begin{array}{l}\text { SAFP(103.004) } \\
\text { Requirement Reference: SNF-RD.PM-O01 Rev 0, Seclion } 5.3 .4 \\
\text { Canislerization. The SNF program shall undertake a joinl eflort with RW to ulitize a standardized canister, developed by RW, for storing, } \\
\text { itansporting, and disposing of DOE-Owned SNF. }\end{array}$ \\
\hline 19 & 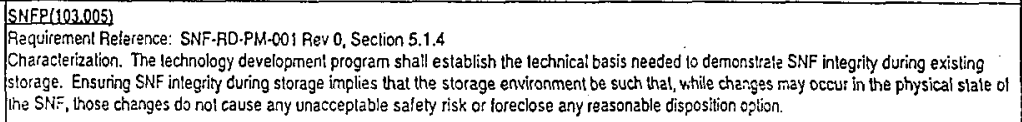 \\
\hline 20 & $\begin{array}{l}\text { SNFP(103,006) } \\
\text { Requicement Relerence: SNF-RO-PM-001 Rev 0, Section } 5.2 .6 \\
\text { Characterization. The lechnology developmenl program shall establish technical bases tor interin slorage and permanent disposal of DOE-owned } \\
\text { SNF, and shall develop criteria and procedures lo ensure thal the SNF and facilities satisty the technical bases. }\end{array}$ \\
\hline 21 & $\begin{array}{l}\text { SNFP(103.007 } \\
\text { Requiremen!Relesence: SNF.RD.PM.001 Rev 0, Section } 5.2 .2 \\
\text { Codes and Standards. New SNF facilities shall be designed, consiructed, and operated to modem commerciel industry codes and slandards. }\end{array}$ \\
\hline 22 & 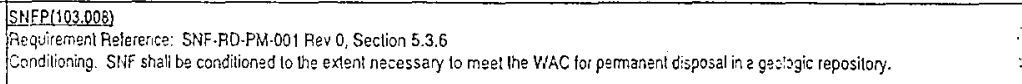 \\
\hline 23 & 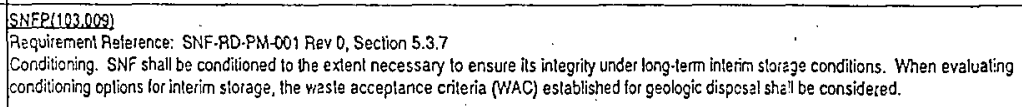 \\
\hline 24 & 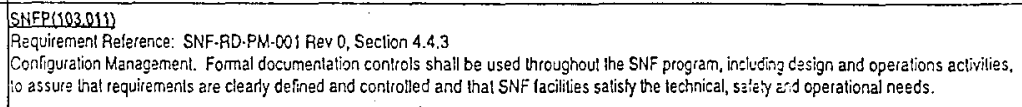 \\
\hline 25 & 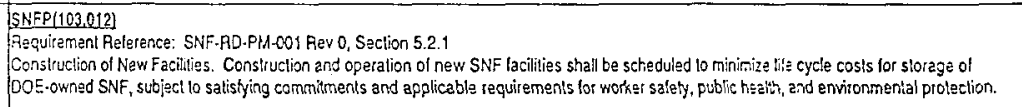 \\
\hline 26 & 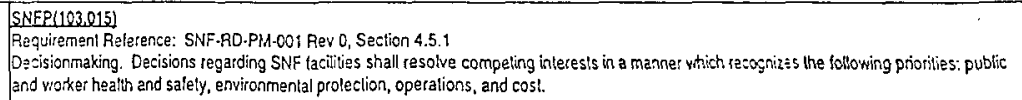 \\
\hline
\end{tabular}


Table 1.6.3.1-1 Condition, Store, and Disposition SNF Material Requiremenits (Continued)

\begin{tabular}{|c|c|}
\hline 27 & $\begin{array}{l}\text { SNEP(103.016) } \\
\text { Requitement Reierence: SNF-RO-PM-001 Rev 0, Section } 5.2 .10 \\
\text { Decontamination and Decommissioning. New SNF facilities shall be designed, constructed, maintained, } \\
\text { and operaled to facilitate eventual deconlamination and decommissioning. }\end{array}$ \\
\hline 28 & 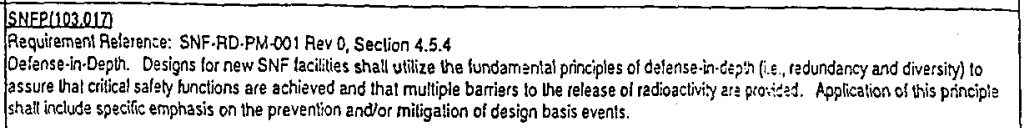 \\
\hline 29 & 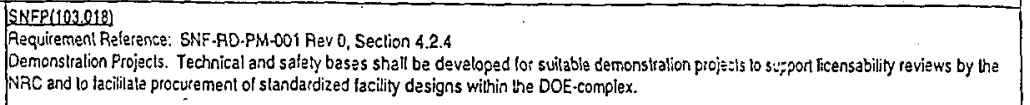 \\
\hline 30 & $\begin{array}{l}\text { SNFP(103.019) } \\
\text { Requiremenl Relesence: SNF-FD.PM-001 Rev 0, Section } 5.2 .3 \text {. } \\
\text { Design Lile. New SNF lacilities shail be designed for a minimurn of life of } 40 \text { years. }\end{array}$ \\
\hline $3 i$ & 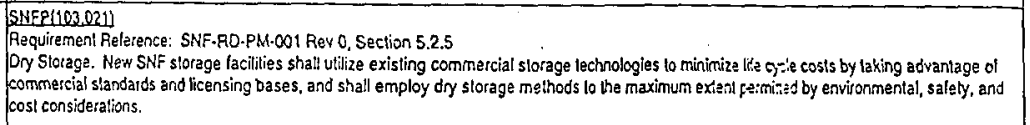 \\
\hline 32 & 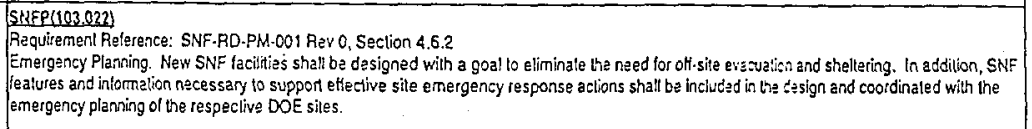 \\
\hline 33 & 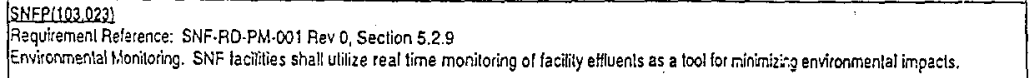 \\
\hline 34 & 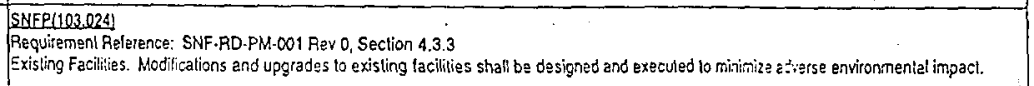 \\
\hline 35 & 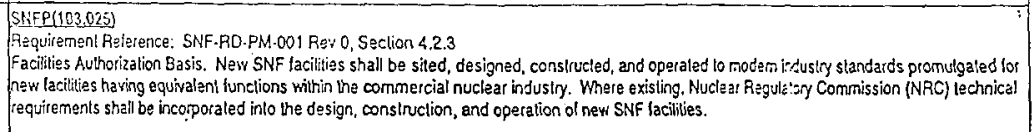 \\
\hline 36 & 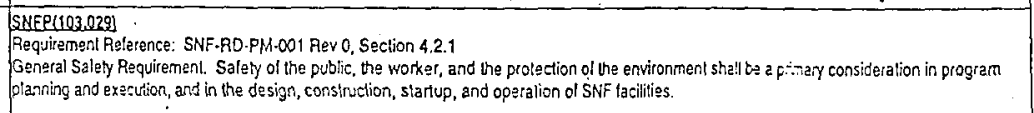 \\
\hline 37 & 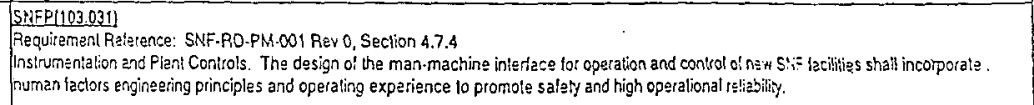 \\
\hline$\sqrt{38}$ & 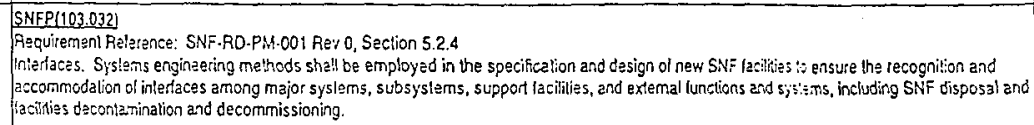 \\
\hline
\end{tabular}


Table 1.6.3.1-1 Condition, Store, and Disposition SNF Material Requiremenis (Continued)

\begin{tabular}{|c|c|}
\hline 39 & $\begin{array}{l}\text { SNFP(103.0351 } \\
\text { Requirement Relerence: SNF.AD.PM.001 Aev 0, Section 4.7.1 } \\
\text { Waintenance. New SNF facilities shall have ready access to equipment, provide support equipment located } \\
\text { and sized to facilitate work, assure that systems and components have high reliability, use automation when } \\
\text { cost effeclive and salety enhancing, and provide for mockups and training aimed ai reducing radiological } \\
\text { exposure and easy repair or replacement of components. Preventative maintenance approaches shall } \\
\text { receive primary locus when undertaking facility maintenance planning. }\end{array}$ \\
\hline 40 & 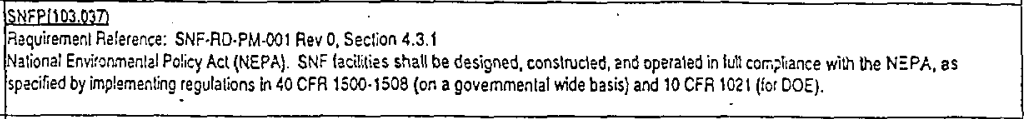 \\
\hline A1 & 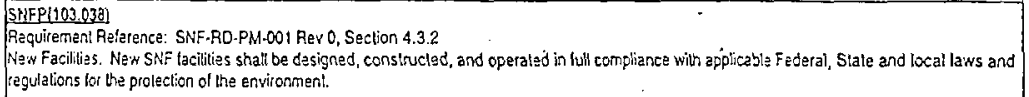 \\
\hline 42. & 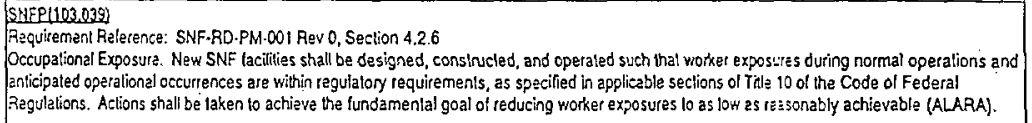 \\
\hline 43 & 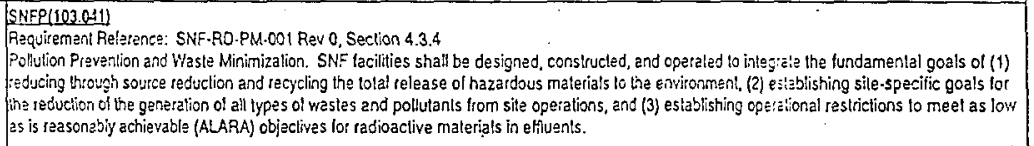 \\
\hline 44 & 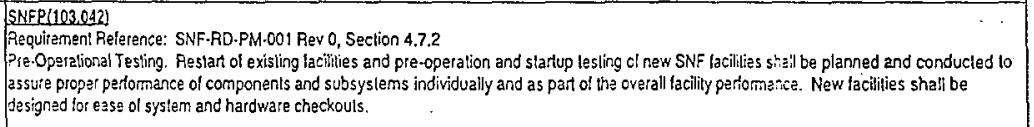 \\
\hline 45 & 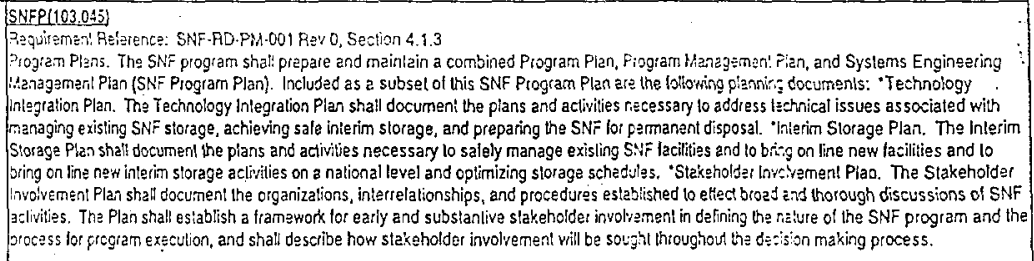 \\
\hline 46 & 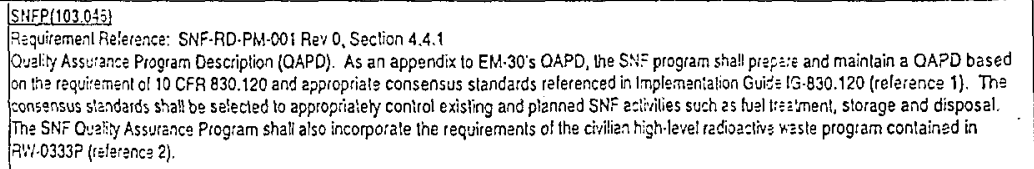 \\
\hline 47 & 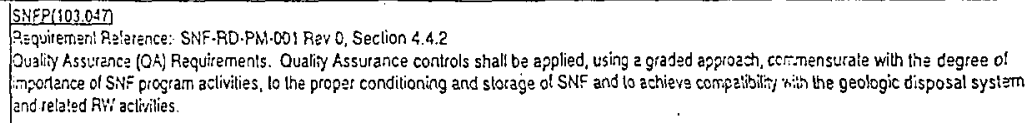 \\
\hline
\end{tabular}


Table 1.6.3.1-1 Condition, Store, and Disposition SNF Material Requirements (Continued)

\begin{tabular}{|c|c|}
\hline 48 & $\begin{array}{l}\text { SNFP(103.048) } \\
\text { Requirement Relerence: SNF-RD-PM-DO1 Rev 0, Section } 4.2 .2 \\
\text { Regulatory Compliance. SNF facillies shall be designed, constructed, and operaled in tell compliance with applicable Federal, State and local laws } \\
\text { and reguiations lor the prolection of the public and worket health and salely. }\end{array}$ \\
\hline 49 & 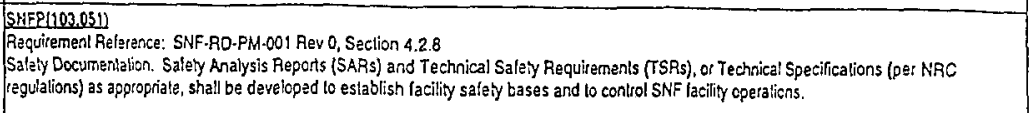 \\
\hline 50 & $\begin{array}{l}\text { SNFP(103.061) } \\
\text { Aaquitement Relerenc: SNF-RO.PM-001 Rev } 0, \text { Seclion } 4.5 .5 \\
\text { Sile Related Hazards. SNF facilities shall be designed to commercial industry slandards lor resistance to saismic evenls, floods, winds, and olher } \\
\text { nalural phenomena. }\end{array}$ \\
\hline 51 & 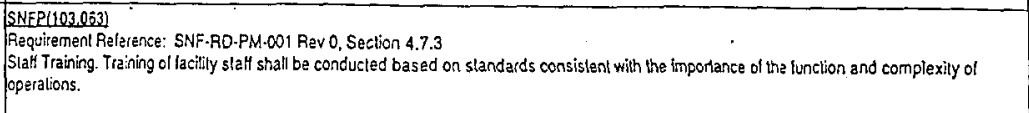 \\
\hline 52 & $\begin{array}{l}\text { SHEP(103064) } \\
\text { Requiremenl Relerence: SNF-RO-PM-001 Rev 0, Section } 4.7 .3 \\
\text { Slatt Training. A stall training program shall be implemented to meel either DOE or nuclear power indusiry slandards, as appropriale for the } \\
\text { panticular operation. Relraining and recentification shall be part of the operalor training plan. }\end{array}$ \\
\hline 53 & 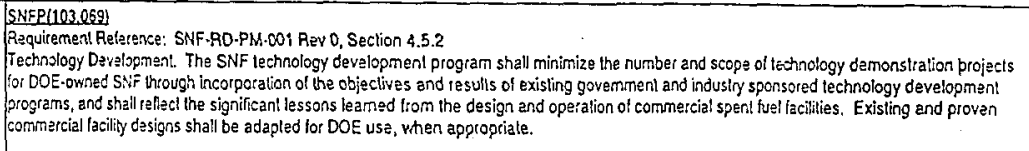 \\
\hline 54 & 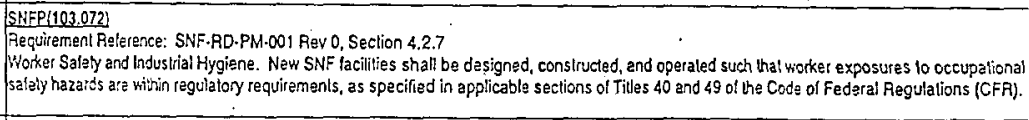 \\
\hline 55 & 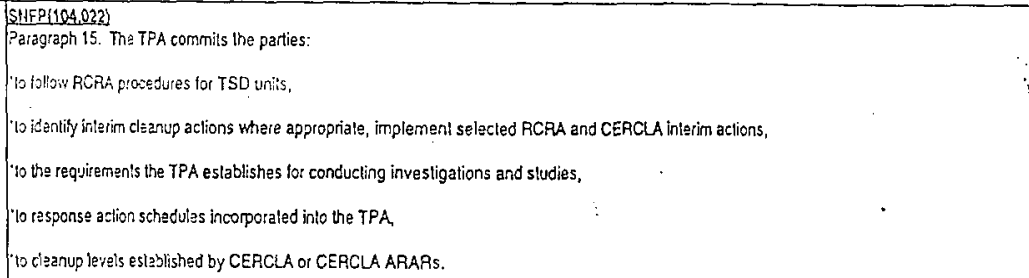 \\
\hline 56 & 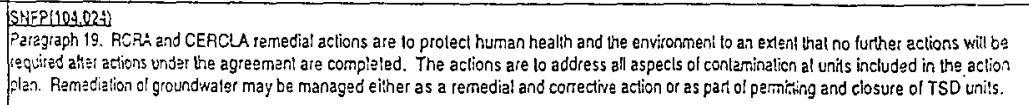 \\
\hline
\end{tabular}

\subsubsection{Dispose of SNF Disposition System}

\subsubsection{Deaclivale SNF (Other) 400 Area}

1.6.4.2 Deactivate Hol Vacuum Conditioning System 
1.6.4.3 Deactivale Canister Storage Building (CSB)

\subsubsection{Deaclivate SNF (Other) 200 Area}

\subsubsection{Deactivate Cold Vacuum Drying Syslem}

\subsubsection{Manage K Basin Deactivation System}

\subsubsection{Manage SNF Project}

1.6.5.2 Obtain Public involvement

1.6.6 Acquire K Basin Deactivation System

\subsubsection{Acquire SNF Project Misșion Essential Capabilities}

Table 1.6.6.1-1 Acquire SNF Project Mission Essential Capabilities Requirements

\begin{tabular}{|c|c|}
\hline 1 & 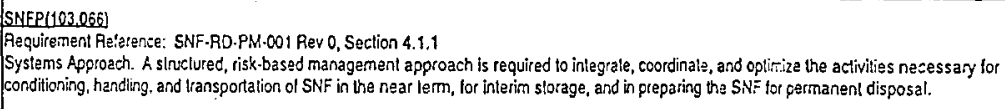 \\
\hline 2 & $\begin{array}{l}\text { SNFP1103.060 } \\
\text { Requirement Relerence: SNF-RD-PM-001 Rev 0, Section } 4.1 .1 \\
\text { Syslems Approch. A systems approach shall be used to support program inlegration and to provide a slruclured, logical, documentable, and } \\
\text { delensible approach lo planning. }\end{array}$ \\
\hline
\end{tabular}

\subsubsection{Operate \& Maintain K Basin Deactivation System}

Table 1.6.7-1 Operate \& Maintain K Basin Deactivation Syslem Requirements

\begin{tabular}{|c|c|}
\hline 1 & $\begin{array}{l}\text { UDD, Heaclors on the River, Salety Objective } \$ 38 \\
\text { Provide survellance and meintenance of the } 1706 \mathrm{KE} K \mathrm{KELKER} \text { Facirity. }\end{array}$ \\
\hline 2 & 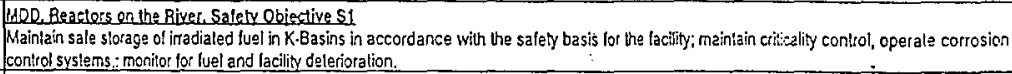 \\
\hline 3 & 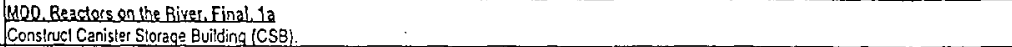 \\
\hline 4 & $\begin{array}{l}\text { BDQ Reactors on the Hiyec. Final. th } \\
\text { Consirucl Cold Vaceum Orying Facility. }\end{array}$ \\
\hline 5 & 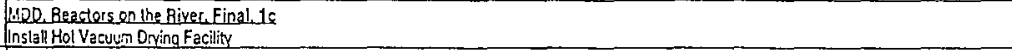 \\
\hline 6 & $\begin{array}{l}\text { MDD, Reactors on the Rivet Final, 1d } \\
\text { Upgrade K.Basin Factilit }\end{array}$ \\
\hline 7 & 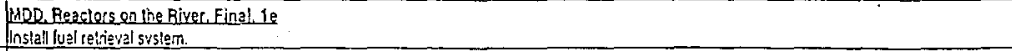 \\
\hline 8 & $\begin{array}{l}\text { MOQ Reactors on the Fiver. Final, If } \\
\text { Fabricaie transogrtition system and casks. }\end{array}$ \\
\hline 9 & $\begin{array}{l}\text { Moo Reaclors on the Biver. Final } 19 \\
\text { Fabricale multi.cenistac overpack contaners. }\end{array}$ \\
\hline 10 & 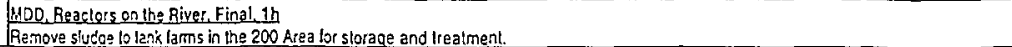 \\
\hline
\end{tabular}


Table 1.6.7.1 Operate \& Maintain K Basin Deactivalion System Requirements (Continued)

\begin{tabular}{|c|c|}
\hline 111 & $\begin{array}{l}\text { MDO, Beactors on the Biver final } 11 \\
\text { Fabricale sludge removal system. }\end{array}$ \\
\hline 12 & $\begin{array}{l}\text { WDQ. Beactors on the River Final.1j } \\
\text { Remove debris for deconlaminalion and disposal. }\end{array}$ \\
\hline 13 & $\begin{array}{l}\text { WoQ Reactoes on the River. Final. ik } \\
\text { Procure debris dacontamination services. }\end{array}$ \\
\hline 14 & 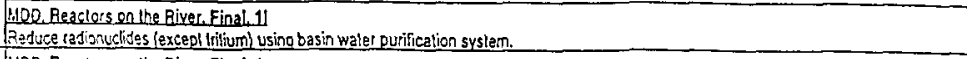 \\
\hline 15 & $\begin{array}{l}\text { MOR Reacters on the Biver Fina:1m } \\
\text { Upgiade ba sins water purificalion system. }\end{array}$ \\
\hline 16 & 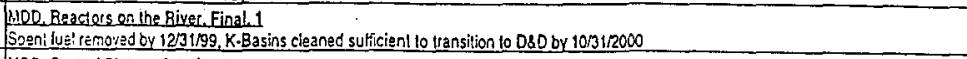 \\
\hline 17 & 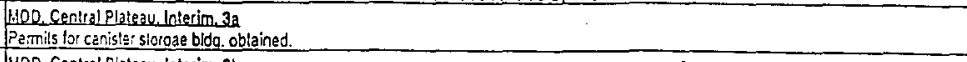 \\
\hline 18 & 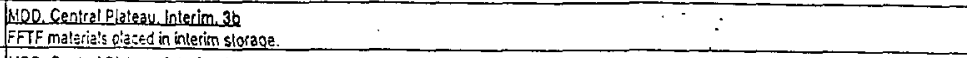 \\
\hline 19 & 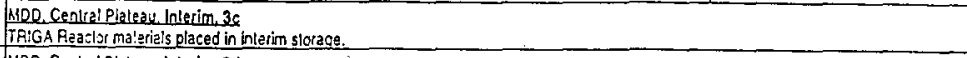 \\
\hline 20 & 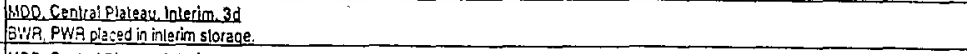 \\
\hline 21 & $\begin{array}{l}\text { Moo Cenleal Plateau Interion. 3e } \\
\text { B.Cell Cleanoul Spetial Case Fuel placed in inlerim slorage. }\end{array}$ \\
\hline 22 & HDQ Central plateau Interim. 31 \\
\hline 23 & $\begin{array}{l}\text { MOD, Central Plateau, interim, } 39 \\
\text { Condition Hol Vacuum Or' spenl fuel. }\end{array}$ \\
\hline 24 & 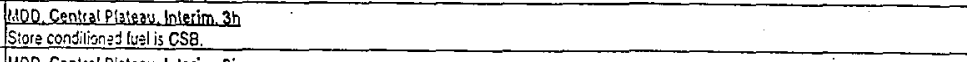 \\
\hline 25 & $\begin{array}{l}\text { M00 Central Plateau Interim, } 3 \mathrm{j} \\
\text { ODlain Nationel Repository wasle and packaning criteria (WAC \& PAC). }\end{array}$ \\
\hline 20 & $\begin{array}{l}\text { Meo Cenlral Pialeav Final, 39a } \\
\text { Assure soanl lue!'s are safe, slable, and 'road.ready". }\end{array}$ \\
\hline 27 & $\begin{array}{l}\text { P.100 Cenlra! Plaleau,Fínal.39b } \\
\text { Provide inle rim siorage. }\end{array}$ \\
\hline 28 & $\begin{array}{l}\text { Wod Central Flaleau. Final } 398 \\
\text { Oblain permils for lransporting off-site. }\end{array}$ \\
\hline 29 & 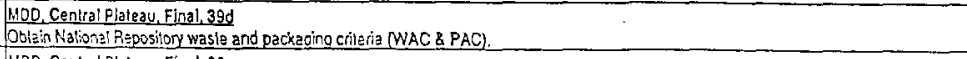 \\
\hline 30 & 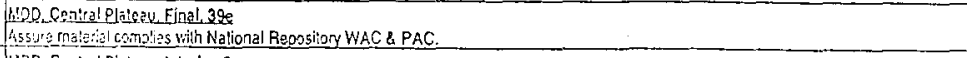 \\
\hline 31 & 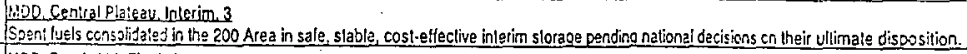 \\
\hline 32 & $\begin{array}{l}\text { 3100 South 600 Fingl as } \\
\text { Transport agoirable FFTF luais to } 200 \text { Inlerin Slorage Area }\end{array}$ \\
\hline 33 & $\begin{array}{l}\text { MOQ South 602 Final } 46 \\
\text { Trensport TRISA Guals to 200 ISA. }\end{array}$ \\
\hline 34 & 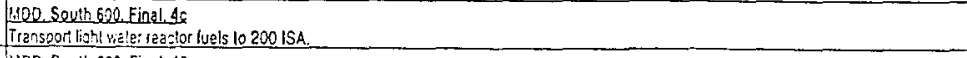 \\
\hline 35 & Hoo, South 600 Final $40 \mathrm{a}$. \\
\hline 35 & 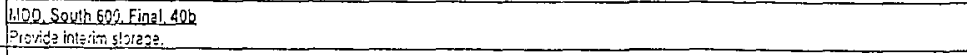 \\
\hline 37 & 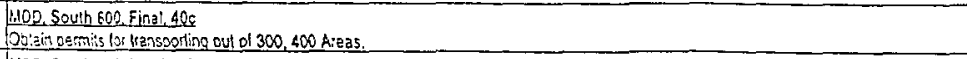 \\
\hline 38 & 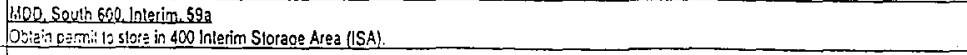 \\
\hline 39 & 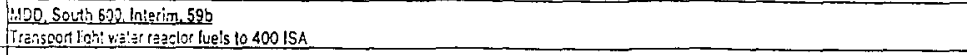 \\
\hline 40 & 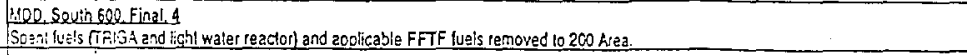 \\
\hline 41 & 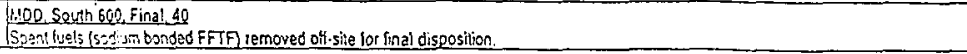 \\
\hline
\end{tabular}


Table 1.6.7-1 Operate \& Maintain K Basin Deactivation System Requirements (Continued)

42 MDQ South 600 Interion 59

Spent fuels (light water reactor) removed to interim storage in 400 Area pending availability of a00 Area inlerim stor 39 e.

\subsubsection{Deactivate K Basins}

Table 1.6.7.1-1 Deactivate K Basins Requirements

\begin{tabular}{|c|c|}
\hline is & 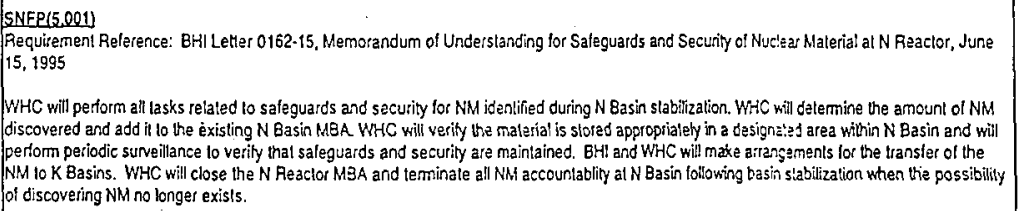 \\
\hline 2 & $\begin{array}{l}\text { SNFP } 6.0011) \\
\text { It is important thal the UCO being designed for SNF be dimensionally compatible with the MPC, such that lor tutu: transport and uttimale disposal } \\
\text { in the Nationat Repository the MCOs could be placed inside MPCs. }\end{array}$ \\
\hline 3 & $\begin{array}{l}\text { SNFP(Z002) } \\
\text { Aequiremen! Relerence: DOE Letter 95-NMD-071. Approval of K Basins Siudge Disposition Strategy. June 13, 1995 } \\
\text { RL directs WHC lo continue } 10 \text { work on dispositioning of the K.East and K.Wes! Basin floor sludges to be sent to etther the Tank Farms or the Solid } \\
\text { Waste Managemenl Factily as appropriate. }\end{array}$ \\
\hline 4 & $\begin{array}{l}\text { SNEP(7.003) } \\
\text { RL direcls WHC to continue dispositioning small pieces of fuel and canister sludges as spanl nuclear hel lo be processed and sent lo dry storage, } \\
\text { along with the rest of the fuel, il those cannol be transferred to Tank Fams or the Solid Waste Managernent Facility. }\end{array}$ \\
\hline 5 & $\begin{array}{l}\text { SNFP(14005) } \\
\text { Requiremenl Relerence: DNFSB } 94-1 \\
\text { Interim measures have and will be taken inciuding installing a cotterdam between the K-East } 8 \text { asin and the reactor discharge chute by April } 1995 \text {. }\end{array}$ \\
\hline 6 & 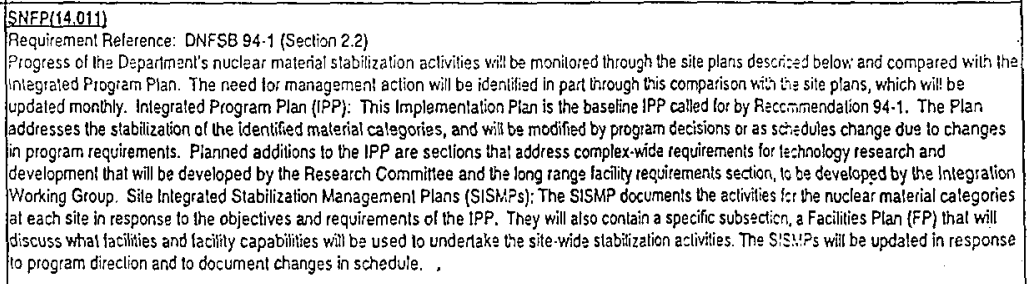 \\
\hline 7 & 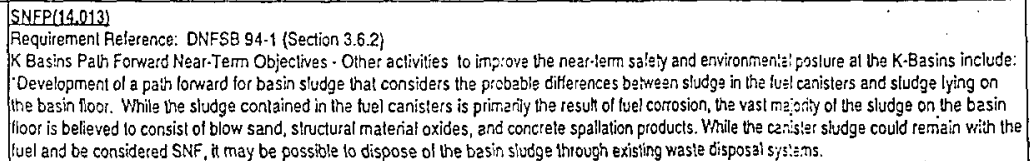 \\
\hline
\end{tabular}


Table 1.6.7.1-1 Deactivate K Basins Requirements (Continued)

\begin{tabular}{|c|c|}
\hline 8 & 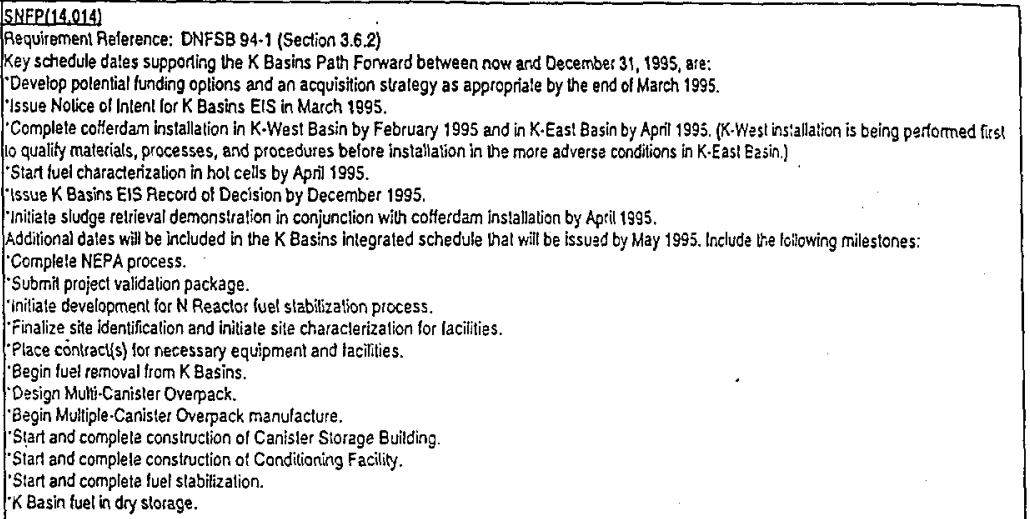 \\
\hline 9 & $\begin{array}{l}\text { SNFP(14.020) } \\
\text { Requisement Relerence: DNFS8 } 94.1 \\
\text { Fuel and sludge characterization in hot cells will begin by April } 1995 .\end{array}$ \\
\hline 10 & 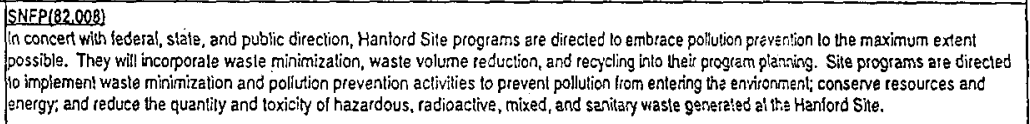 \\
\hline$\sqrt{11}$ & $\begin{array}{l}\text { SHEP(82.014) } \\
\text { Material Management Responsibilities. The Spent Fuel Program is responsible for near.lem sale storage of spent nuclear fuel in the } 105 . \mathrm{K} \text { Basins. } \\
\text { The NEPA process will be used to make a decision of how and where spent nuclear fuel will be managed on the Site. }\end{array}$ \\
\hline 12 & $\begin{array}{l}\text { SNFP182.Q27) } \\
\text { Paragraph 5. Conduct planning and carry out aclivities lo clean out retired facilities to conditions appropriale for lumover to D\&D etionts. }\end{array}$ \\
\hline 13 & $\begin{array}{l}\text { SNFP(B2030) } \\
\text { Site cleanup will be pertormed in accordance with the Tr.Panty Agreemenl (Ecology et el. 1994), as arnended, end olher agteements, and in } \\
\text { compliance with all applicable lederal, slale, and local laws and American indian Ireaty rights. Hanlord Site progrems must also comply with DOE } \\
\text { policies and direclives. }\end{array}$ \\
\hline 14 & 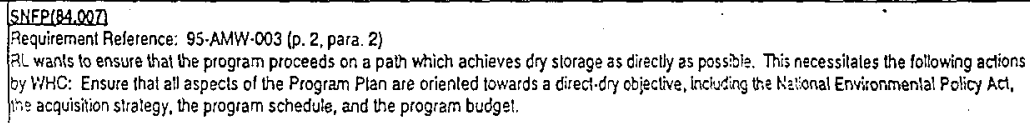 \\
\hline 15 & $\begin{array}{l}\text { SNFP(B4,019) } \\
\text { REquirement Reterence: } 95 \cdot A M W-003(\because 8) \text {. } \\
\text { WHC vill input to } S \text {. Clark on dose assessments for } K \text { Basin and Path Forward activitios. }\end{array}$ \\
\hline$\sqrt{16}$ & 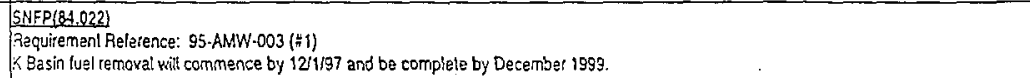 \\
\hline 17 & 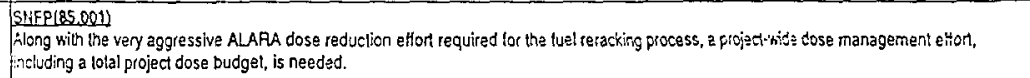 \\
\hline
\end{tabular}


Table 1.6.7.1-1 Deaclivate K Basins Requirements (Conlinued)

\begin{tabular}{|c|c|}
\hline 18 & $\begin{array}{l}\text { SNFP(85.005) } \\
\text { WHC is diracled lo proceed and implemenl the fnlegraled process siralegy. }\end{array}$ \\
\hline 19 & 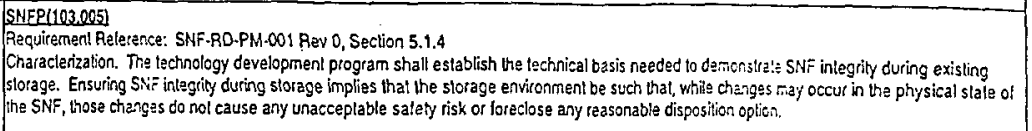 \\
\hline 20 & 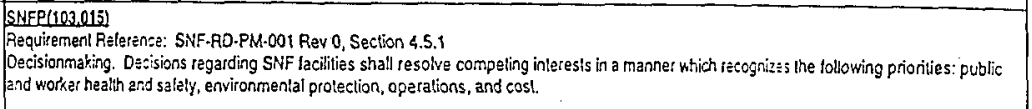 \\
\hline 21 & 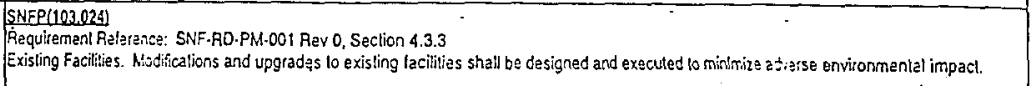 \\
\hline 22 & 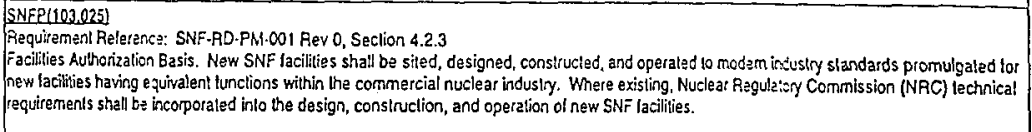 \\
\hline 23 & 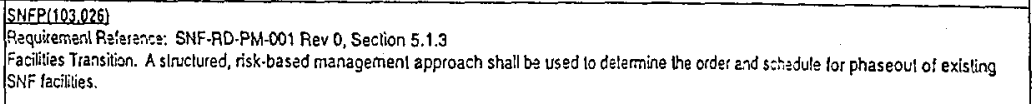 \\
\hline 24 & 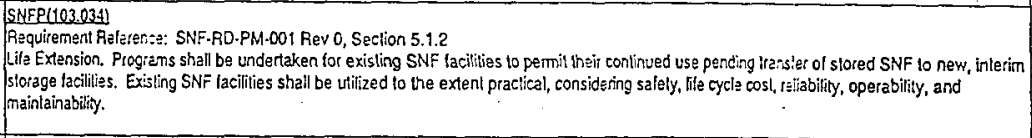 \\
\hline 25 & 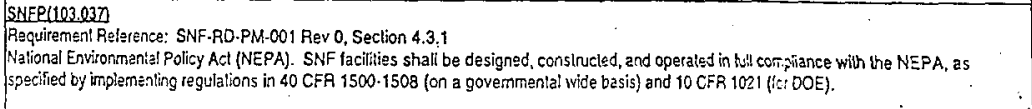 \\
\hline 20 & 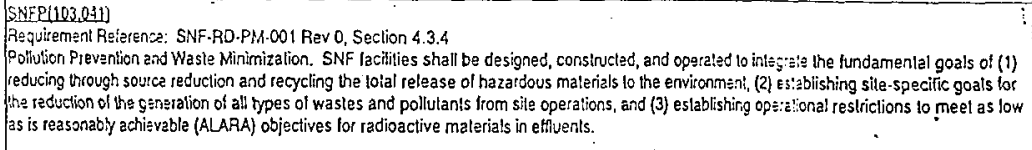 \\
\hline 27 & 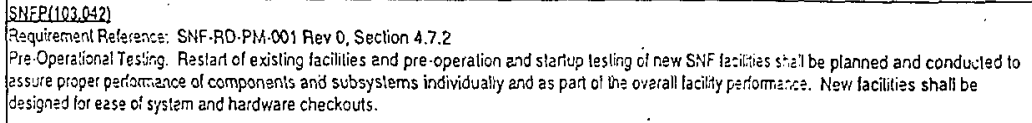 \\
\hline 28 & 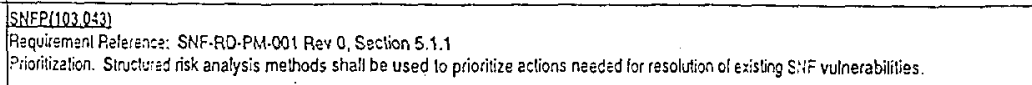 \\
\hline
\end{tabular}




\begin{tabular}{|c|c|}
\hline 29 & 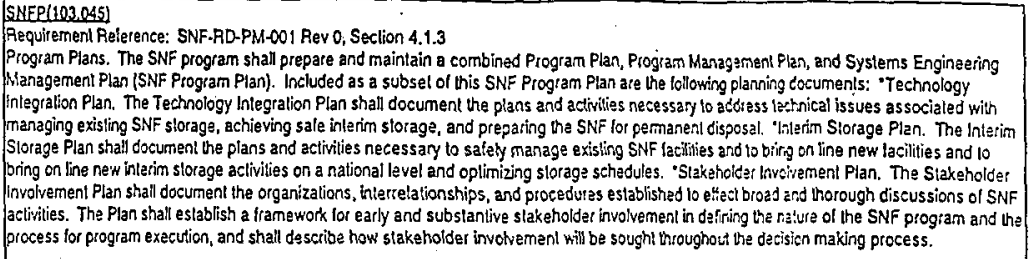 \\
\hline 30. & 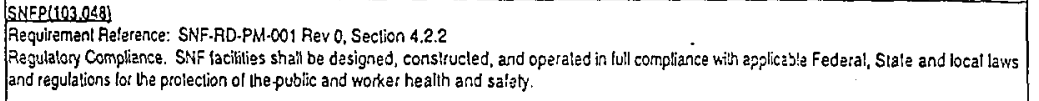 \\
\hline 31 & $\begin{array}{l}\text { SNFP[103.061) } \\
\text { Aequitement Reference: SNF.AD.PN-001 Rev 0, Seclion } 4.5 .5 \\
\text { Sile Relaled Hazards. SNF lacilities shall ba designed to commercial indusly slandards loc tesistance to seismic events, floods, winds, and other } \\
\text { nalural phenomena. }\end{array}$ \\
\hline 32 & $\begin{array}{l}\text { SNFPU103.063! } \\
\text { Aequiremen! Relarence: SNF-RO-PM-001 Rev 0, Section } 4.7 .3 \\
\text { Siaff Training. Training of Iachily slatt shall be conducled based on slandards consislent with the importance of the funclion and complexity of } \\
\text { operalions. }\end{array}$ \\
\hline 33 & 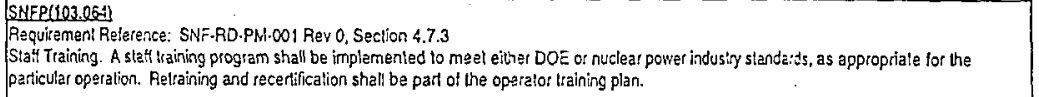 \\
\hline 34 & 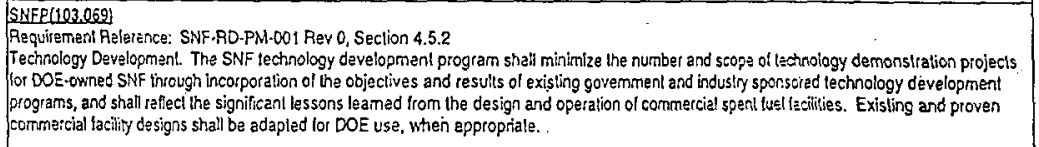 \\
\hline 35 & 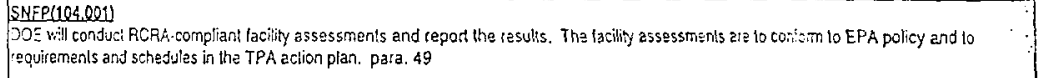 \\
\hline 36 & 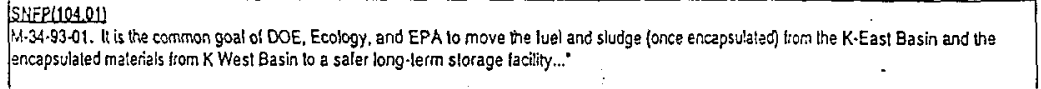 \\
\hline 37 & $\begin{array}{l}\text { SHFP(104.02) } \\
\text { Paragraph 130. To signity completion ol required response actions for an operable unit, the lead Izgutalon agerng for the unit will issue a notice of } \\
\text { completion to DOE. The tead agency may is sue a notice of completion for partial complation of response astions is: a unit. }\end{array}$ \\
\hline 38 & $\begin{array}{l}\text { SHEP(1040021 } \\
\text { DOE vill conduct RCRA.compliant facility investigations and report the results. The lactity investigations ait } 10 \text { ce-tiom lo EPA policy and } 10 \\
\text { requirsments and schedulas in the TPA action plan. para. } 51\end{array}$ \\
\hline 39 & $\begin{array}{l}\text { 5MFP(104,03) } \\
\text { Paragraph 54. DOE will propose remedial actions or corrective actions which conlom to EPA polizy and lo requitenenls and schedules in the TPA } \\
\text { action plan. }\end{array}$ \\
\hline 40 & $\frac{S N F P[104.005]}{M \cdot 34 \cdot 00 \cdot T 01 . \text { Issue Nolice ol Intent lor N.Reaclor Fuel EIS, Due Date: } 6^{\prime} 30 / 94}$ \\
\hline
\end{tabular}


Table 1.6.7.1-1 Deactivate K Basins Requirements (Continued)

\begin{tabular}{|c|c|}
\hline 41 & $\begin{array}{l}\text { SMEP(104.006) } \\
\text { M-34.00-T04. Submit a schedule describing aclivities for the final disposition of contaninated K. Easl basin walar lot planning purposes to support } \\
\text { the 100-KR-4 record of decision. Due Date: } 10 / 31 / 94\end{array}$ \\
\hline 42 & $\begin{array}{l}\text { SNFP(104.007) } \\
\text { M-34.00-T05. DOE shall provide a schedule for tuel and sludge encapsulation and contaminaled water removal ct replacement } 10 \text { Ecology and } \\
\text { EPA that supports the TPA miteslone. Due Date: } 3 / 31 / 95\end{array}$ \\
\hline 43 & 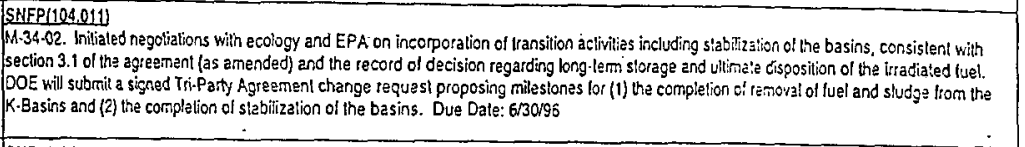 \\
\hline 44 & 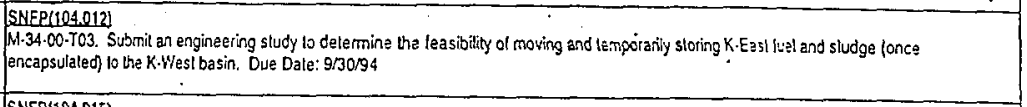 \\
\hline 45 & $\begin{array}{l}\text { SNEP(104.015) } \\
\text { Paragraph 101. It the ragulalors conciude that additional work or modificalions to planned work are necessary, has are to notity DOE. DOE is lo } \\
\text { assess is ability to partom the newly identified work, and inlorm the regulators of its evalualion. }\end{array}$ \\
\hline 46 & SNFP(104.019) \\
\hline 47 & 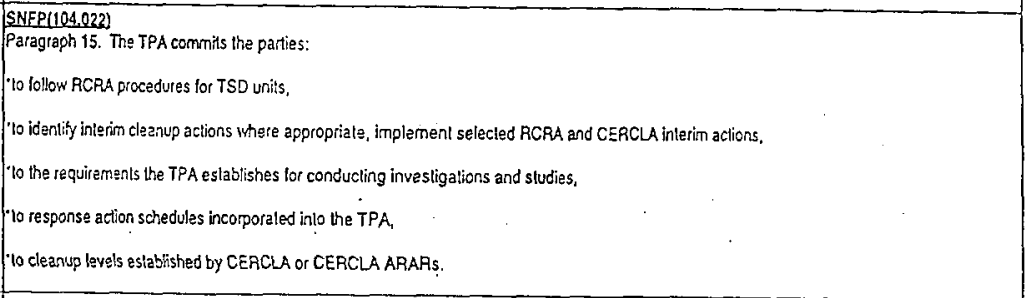 \\
\hline 48 & 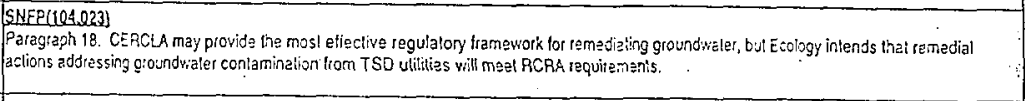 \\
\hline 49 & 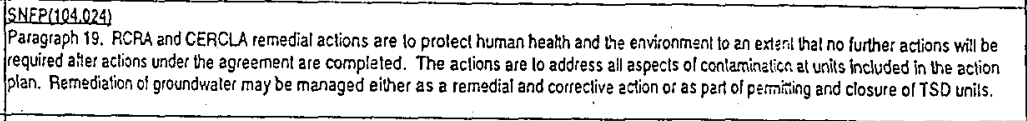 \\
\hline 50 & $\begin{array}{l}\text { SNFPU104.020 } \\
\text { Paragraph } 49 \text {. DOE will conduct CERCLA-compliant remedial investigations and report the results. The remedizi invesligations are to conlorin to } \\
\text { EPA policy and to requirements and schedules in the TPA action plan. }\end{array}$ \\
\hline 51 & $\begin{array}{l}\text { SNFP(104,029) } \\
\text { Paragraph 53. COE will undertake RCRA-compliant corrective measures sludies and report the resul's. The leci:..ij essessments are to contom to } \\
\text { EPA policy and to taquiernents and schedules in the TPA action plan. }\end{array}$ \\
\hline 52 & 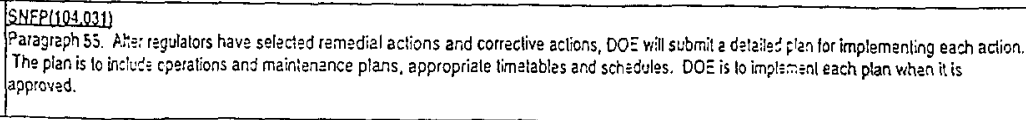 \\
\hline
\end{tabular}


Table 1.6.7.1-1 Deactivate K Basins Requiremenis (Continueo;

\begin{tabular}{|c|c|}
\hline 53 & 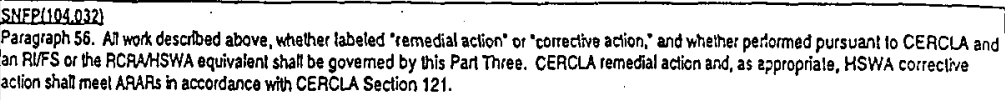 \\
\hline 54 & $\begin{array}{l}\text { SNEP(104033) } \\
\text { Paragraph 90. The TPA is nol inlended lo produce cleanup actions which make other actions less elfeclive. [Rrities XXV and XXVI centcint } \\
\text { procedures lor resolving circumstances where actions appear physically inconsislenl.] }\end{array}$ \\
\hline 55 & 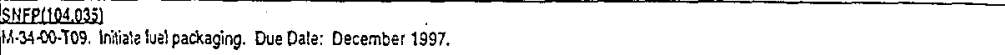 \\
\hline 56 & SNFP(104035) \\
\hline 57 & 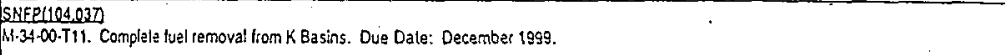 \\
\hline 58 & $\begin{array}{l}\text { SHEP(104.038) } \\
\text { M-34.00-T12. Is sue a plan including schedule for complation ol fuel stabilization. DOE will submit a signed Tri.Pa.ty Agraement Changa Reques! } \\
\text { proposing new milestones. Due Dale: Seplember } 1995 \text {. }\end{array}$ \\
\hline 59 & SHEP(104.039) \\
\hline 60 & SNEP(104.040) \\
\hline 61 & $\begin{array}{l}\text { SNFP(104.041) } \\
\text { W.34.00.T15. Complale bulk sludge removal from K Basins. Due Dale: December } 2000 .\end{array}$ \\
\hline 62 & $\begin{array}{l}\text { SNEP(104042) } \\
\text { M-34-00-716. Conplate debris removal from K Basins. Due Date: December } 2002 .\end{array}$ \\
\hline 63 & $\begin{array}{l}\text { SHEP }(104.043) \\
\text { M.34.00-317. Complete RemovaVDisposal ol Contaminaled Debris in K Basins. Due Dale: Decernb=r } 2001 .\end{array}$ \\
\hline 64 & $\begin{array}{l}\text { SUEP(104.044) } \\
\text { [1-34-00-T05. Complete development of Unit Wanager recommendalions regarding K Basins. Due Date: March } 1395 .\end{array}$ \\
\hline$\widehat{005}$ & M. 34.03 . Complete removal of fuel and sludge from the K Besins. Due Dale: December 2002. \\
\hline
\end{tabular}

\subsubsection{Dispose of K Basin Deactivation System}

\subsubsection{Perform K Basins Deaclivation}

Table 1.6.8.1-1 Pertorm K Basins Deactivation Requirements

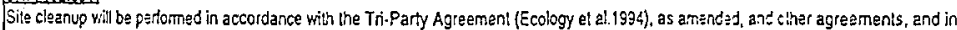
compliance with all applicable federal, stale, and local laws and American Indien treaty rights. Hanlord Sita progi policies and directives. 


\section{7-PROJECT ISSUES AND ASSUMPTIONS}

Table 1.7-1 contains the issues that affect the project. These include projecl specific issues, as well as the site-level issues that have been assigned to the project for resolution. It also contains the assumptions that are used as a basis for the development of project plans until the issues are formally resolved with records of decision. The "Champion" column determines if the Project has lead responsibility or is an affected participant. If the champion belongs to the Project, the Project has the lead. If not, the Project is an aflected participant. Project plans include appropriate activities and resources for resolving these issues.

MOTE: Please see tables in section 5.0 . 


\section{SPENT NUCLEAR FUEL PROJECT WBS 1.4.1}

HNF-SP-1104 Rev 4

\section{WBS HIERARCHY}

The Project Authorization Documents (PADs) are the contractual work authorization between Fluor Daniel Hanford, Inc. (FDH) and their major subs. (These PADs are the equivalent extension of the work authorization that DOE issues to FDH.) The PADs are written in accordance with the (FDH/Duke Engineering \& Services Hanford, Inc. (DESH) contract, reflect common FDH/DESH management strategies and are consistent with the Spent Nuclear Fuel (SNF) Project Management Plan (PMP).

The previous Westinghouse Hanford Company WBS numbering system, specifically at the subproject level (WBS 5) does not conform, both vertically and horizontally, to the PMP, Configuration Control, schedule roll-up or the management strategy employed in the Rev. 0 MYWP. At the Cost Account Level (CA-WBS Level) the individual blocks were found to be adequate to support the management strategies and requirements described above, if realigned. This realignment can take place one of two ways;

(1) Completely renumber the financial data system, its history and assign new cost account codes to all cost inputs (i.e., payroll, POs, contracts, etc.), or

(2) Realign the existing WBS Level 6 Cost Accounts to conform with the requirements via use of the PADs.

To minimize impact to the Project, the second option was selected and is represented in the MYWP baselines. 


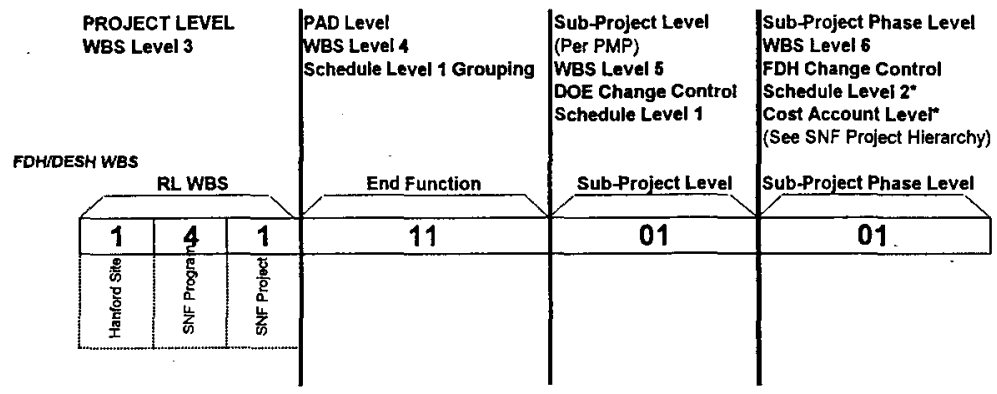




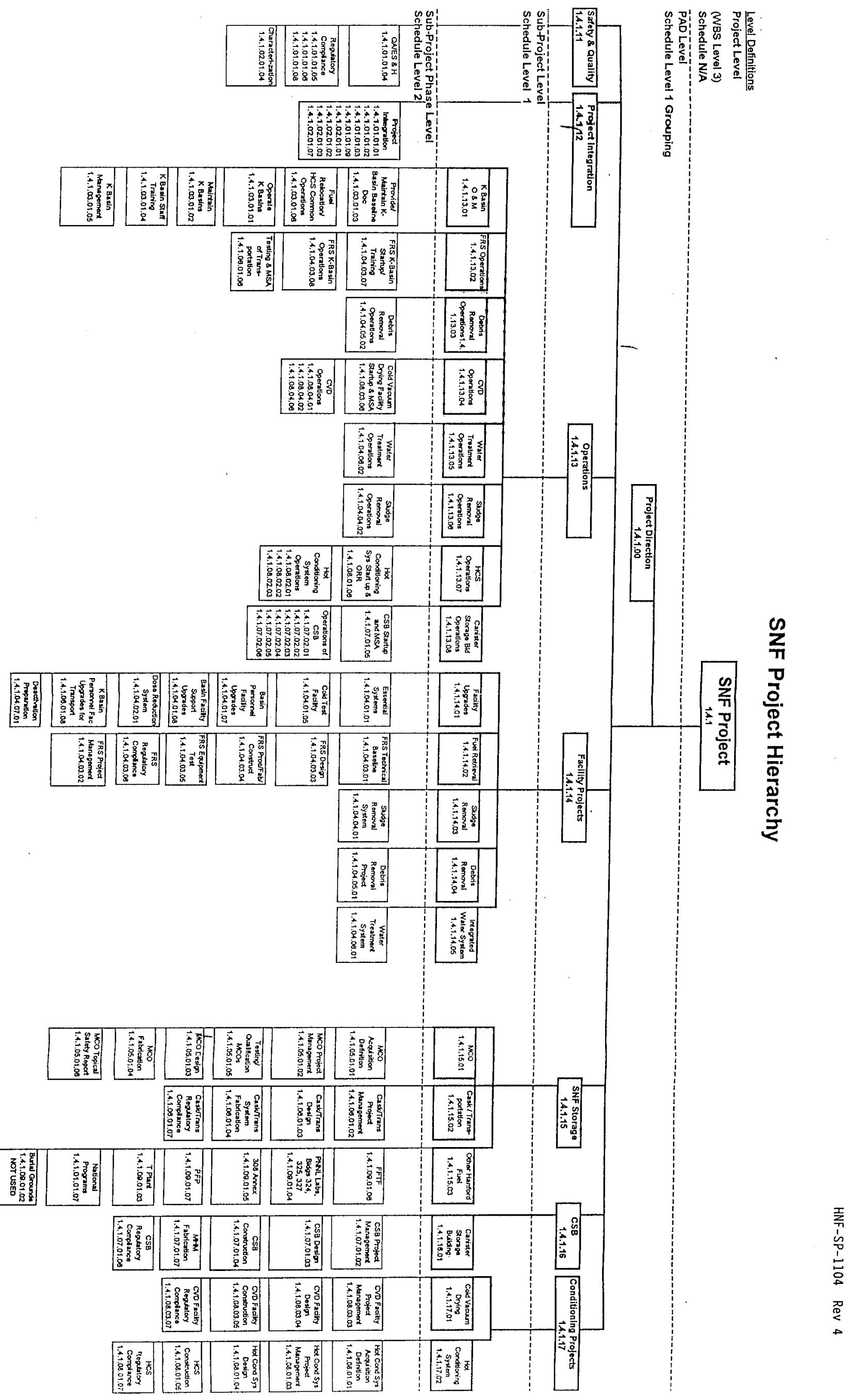




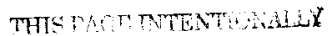
i. BLNE 


\section{SPENT NUCLEAR FUEL PROJECT \\ WBS 1.4.1}

HNF-SP-1104, Rev 4
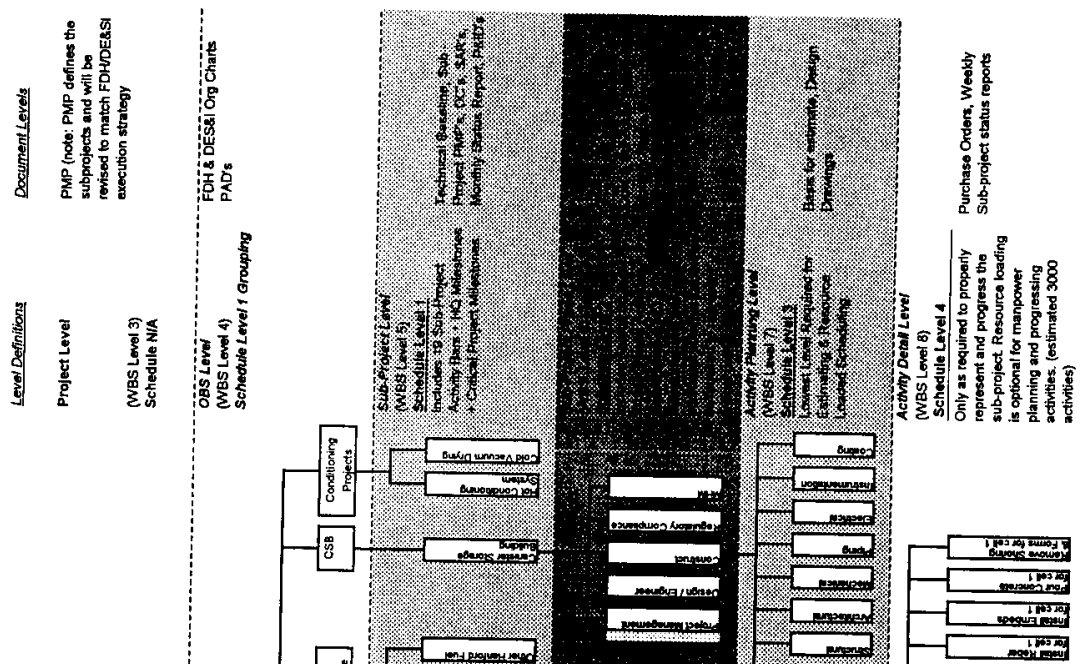

홍
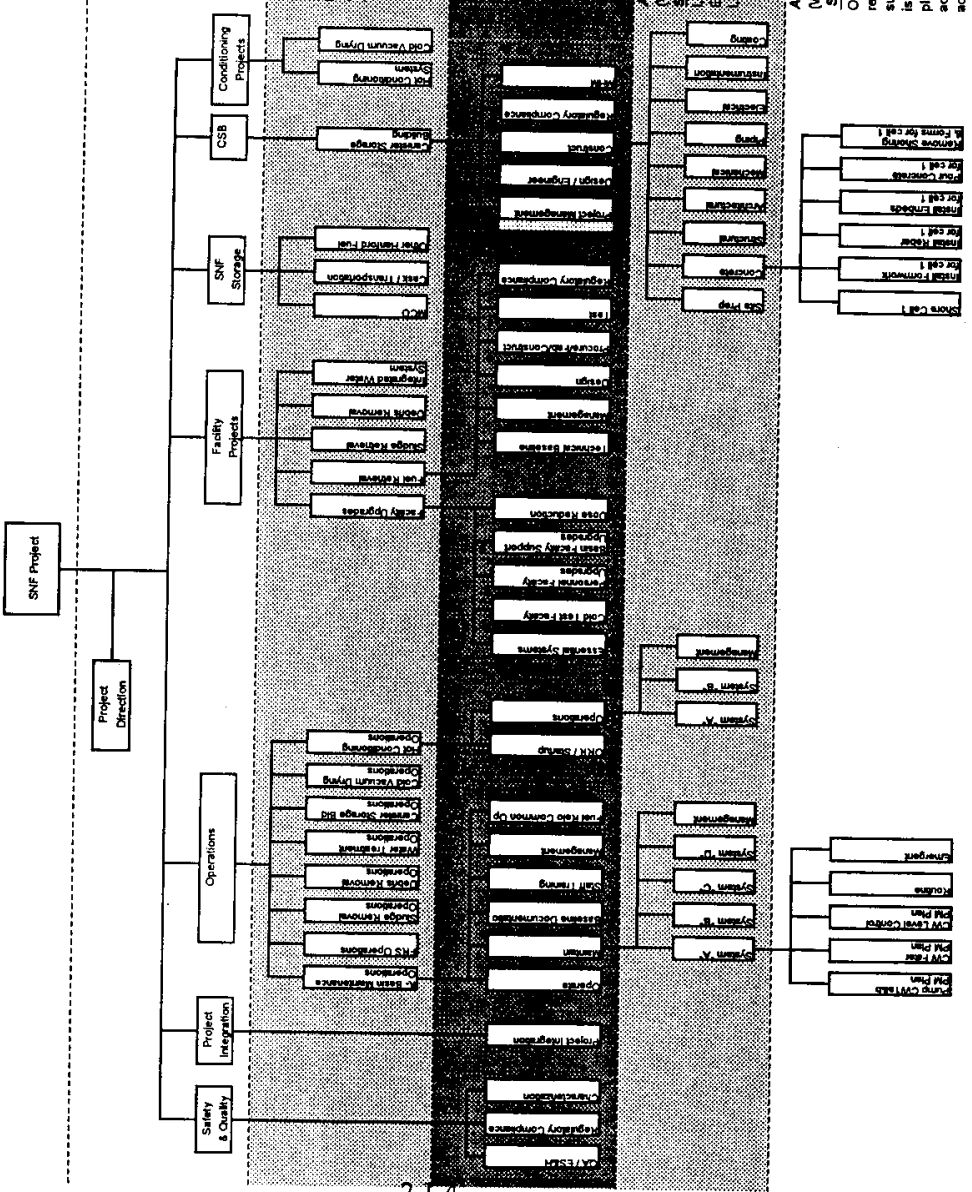
This page intentionally left blank. 
WBE LEVEL 4 DICTIONARY

\begin{tabular}{|c|c|c|}
\hline \multicolumn{3}{|c|}{ WBS DICTIONARY } \\
\hline $\begin{array}{l}1 \text { PROJECT TITLE/PARTICIPANT } \\
\text { Spent Nuclear Fuel Project }\end{array}$ & $\begin{array}{l}2 \text { DATE } \\
\text { January 14, } 1997\end{array}$ & \begin{tabular}{|c}
$\begin{array}{l}3 \text { mENTIFICATION } \\
\text { NO. } \\
14100 \\
\end{array}$ \\
\end{tabular} \\
\hline $\begin{array}{r}4 \text { PROJECT ELEMENT CODE } \\
1.4 .1 .00\end{array}$ & \multicolumn{2}{|c|}{5 PROJECT ELEMENT TITLE } \\
\hline 6 INDEX LINE NO. & $\begin{array}{l}7 \text { REVISION NO AND } \\
\text { AUTHORIZATION }\end{array}$ & 8 DATE \\
\hline \multicolumn{3}{|l|}{9 APPROVED CHANGES } \\
\hline 10 SYSTEM DESIGN DESCRIPTION & \multicolumn{2}{|c|}{$\begin{array}{c}11 \text { BUDGET AND REPORTING NUMBER } \\
\text { EW7040000 }\end{array}$} \\
\hline
\end{tabular}

\section{A. STATEMENT OF WORK}

\section{Work To Be Performed}

The Spent Nuclear Fuel Project Director is the key point of contact for the major subcontractor and is the designated FDH Contracting Officer's Representative (COR). The responsibilities of the project director's group incude the following:

- Lead the development of life-cycle baseline, multi-year, and current fiscal-year plans; integrating the subcontractor into the planning process; and ensuring Sitewide integration of these activities from a scope, schedule, interface, and resource perspective

- Interface with DOE-RL and stakeholders regarding incorporation of regulatory commitments and other requirements 


\section{SPENT NUCLEAR FUEL PROJECT \\ WBS 1.4.1}

HNF-SP-1104, Rev 4

- The prioritization of work activities to accomplish project results on the basis of DOERL's Integrated Priority List and budget direction supported by risk/benefit assessments that consider safety, environmental, technical, and cost factors

- Serving as COR to provide technical and administrative direction, to review the subcontractors project work for compliance, to monitor progress, and to verify status and completion of performance measures

- Integrate project activities in PHMC by facilitating effective communications and resolving issues among projects, ES\&H, PHMC subcontractors, PHMC support organizations, management, and DOE-RL clients.

The Sub-project Phase I elements are as follows: $\quad 1.4 .1 .00$

Major end-item deliverables (related to milestones and interfaces appropriate to this level of the sub-project):

All deliverables as listed in the WBS Dictionaries. 
WBS LEVEL 4 DICTIONARY

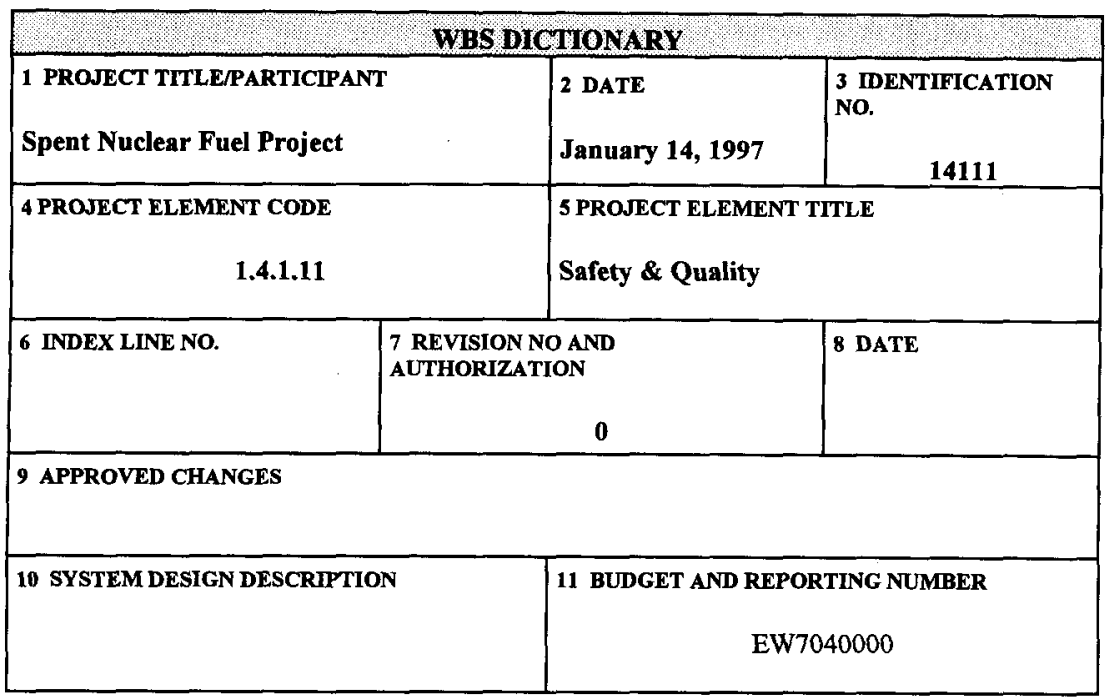

\section{A. STATEMENT OF WORK}

\section{Work To Be Performed}

The Project Safety and Quality WBS Level 4 element encompasses the development/maintenance of QA, Regulatory and Characterization plans and supporting documents associated with the safe, environmentally acceptable, regulatory compliant, and cost effective interim storage for Hanford's spent nuclear fuel consistent with the DOE complex spent fuel disposition plans and in compliance with state and federal regulations.

The scope includes management, administration and integration of SNF project activities associated with environmental, safety and healthy, quality; regulatory compliance, and characterization.

Safety and Quality consists of three main sub-elements at the sub-project level. 


\section{SPENT NUCLEAR FUEL PROJECT \\ WBS 1.4.1}

HNF-SP-1 104, Rev 4

Quality Assurance/ES\&H - develop and maintain Spent Nuclear Fuel Project Quality Assurance Program Plans (QAPP) consistent with regulatory and contractual requirements, conduct assessments to assure compliance to the QAPPs. At this time OCRWM QA requirements are not included. Ensure a consistent approach to NEPA, CAA, and other environmental regulatorydriven documentation for all facilities containing Spent Nuclear Fuel. Preparation and Review is provided for all necessary environmental documentation. Tri-Party Agreement (TPA) negotiations and status activities relative to spent fuel issues are also covered here.

Regulatory Compliance - develop \& maintain SNF Project regulatory policy, strategy, and coordinate/support regulatory reviews. This includes supporting funding for key Regulatory Requirement Team Members and support for Independent Review Panel activities. Also ensures a consistent approach to regulatory documentation for all facilities within the SNF Project. Prepare and maintain the Integrated Safety Management Plan (ISMP), per DNFSB 95-2, which outlines how safety will be integrated, including scheduling data for SARS, permits, etc. Other cross-cutting regulatory plans will also be developed and implemented as required.

Characterization - collect samples of fuel and sludge from the KE and KW Basins; conduct analyses; provide evaluated data describing the physical, chemical, and radiological characteristics of the fuel and sludge to analytical lab and hot cells for analysis; and conduct tests as necessary to support resolution of technical issues. Furthermore, these data will likewise be used to support products or processes such as MCOs, transportation, conditioning and storage.

The Sub-project Phase 1 elements are as follows:

QA/ES\&H (1.4.1.01.01.04)

Regulatory Compliance (1.4.1.01.01.05, 1.4.1.01.01.06, 1.4.1.01.01.08)

Characterization (1.4.1.02.01.04)

Major end-item deliverables (related to milestones and interfaces appropriate to this level of the sub-project):

- Quality Assurance Plans and Project Quality Assurance surveillances.

- Regulatory Strategy Report, Price Anderson Amendments Act plan, Integrated Safety Management Plan (ISMP) and central database of regulatory requirements.

- Development of supporting State/Federal Regulatory permits and applications. 


\section{SPENT NUCLEAR FUEL PROJECT \\ WBS 1.4.1}

HNF-SP-1104, Rev 4

- Sub-project supporting safety analysis such as PSE, PSAR, Phased SAR's and FSAR's as required

- Characterization Management Plan

- Complete sampling, analyses and reporting of $\mathrm{KE} / \mathrm{KW}$ canisters

- $\quad$ Complete the 2nd KW Fuel Sampling, the non-destructive examination of 2nd Fuel

Samples and the canister sludge sampling, analyses and reporting data

- Complete examinations and analyses of the KE fuel and report, and the KE canister sludge and report

- K Basins Fuel and Sludge sampling and laboratory analysis consisting of acquiring fuel/sludge samples from $\mathrm{KE} / \mathrm{KW}$, transportation to Hot analytical labs such as: 327,325 or $222 \mathrm{~S}$ consistent with design/safety requirements. Analysis will include oxidation rates, water content, combustibility and hazardous waste such as PCBs 
WBS LEVEL 4 DICTIONARY

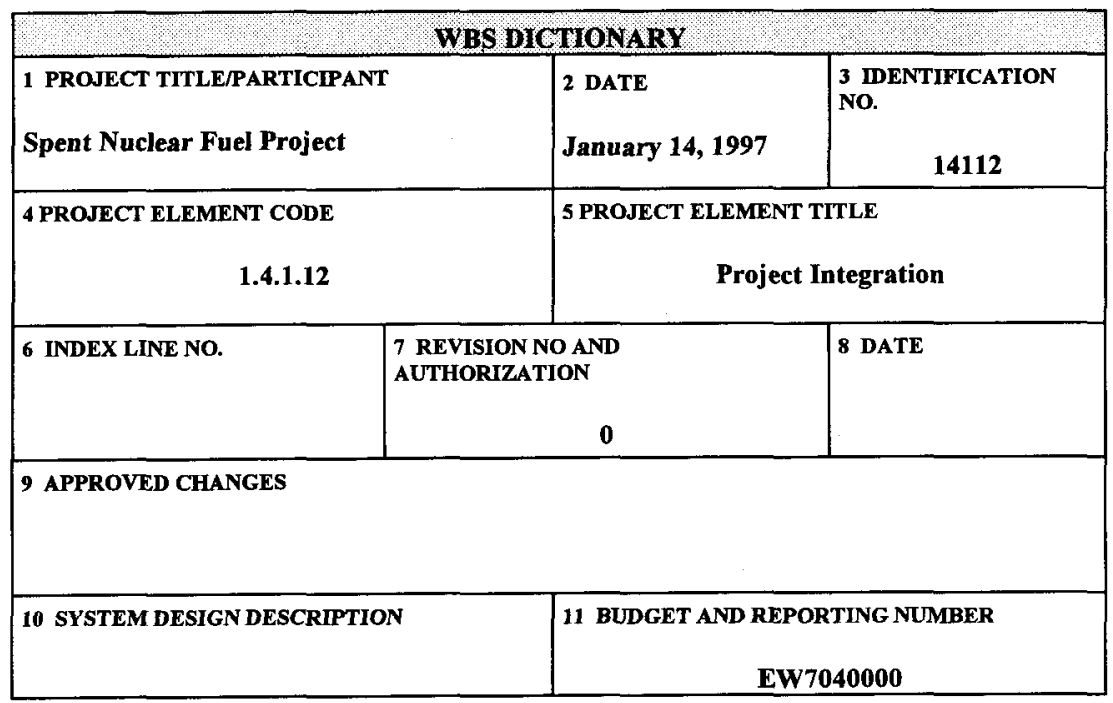

\section{A. STATEMENT OF WORK}

\section{Work To Be Performed}

The Project Integration sub-project element develops, coordinates, and administers the project baseline including the fully-integrated resource based schedule and all changes and revisions to it in a manner which is cost effective, consistent with the DOE complex spent fuel disposition plans and in compliance with state and federal regulations.

The scope includes the program administration, integration and project controls activities required to manage the project. The resource loaded integrated scheduling and budgeting activities provide the cost and schedule baseline. It also, encompasses the overall technical direction for the Spent Nuclear Fuel Project and facilitates the overall technical strategy for the Project and resolution of technical issues as necessary in implement the strategy 


\section{SPENT NUCLEAR FUEL PROJECT \\ WBS 1.4.1}

HNF-SP-1104, Rev 4

The Sub-project Phase 1 elements are as follows:

Project Integration $\quad(1.4 .1 .01 .01 .01,1.4 .1 .01 .01 .02,1.4 .1 .01 .01 .03,1.4 .1 .01 .01 .09$,

$1.4 .1 .02 .01 .01,1.4 .1 .02 .01 .02,1.4 .1 .02 .01 .03,1.4 .1 .02 .01 .07)$

Major end-item deliverables (related to milestones and interfaces appropriate to this level of the sub-project):

- SNF Project cost and schedule baselines, program plans, project control documents, and change management documents.

- Site planning and reporting documents including Multi-Year Work Plans, SMS, and PTS, 10-year Plan and PBS.

- Administrative reports and activities including, significant events, monthly project review packages, all project related documents and correspondence, recognition awards and events.

- Meetings, briefings and reports to stakeholders, Indian tribes, governmental officials, civil entities and the media as requested by project director.

- Revisions to Systems Engineering Management Plan, Project-level Technical Baseline, Interface Control Plan, Configuration Management Plan, and Interface Control Agreements.

- Verification of expected project MCO throughput using commercial modeling software.

- Process throughput capacity and modeling 
WBS LEVEL 4 DICTIONARY

\begin{tabular}{|c|c|c|c|}
\hline \multicolumn{4}{|c|}{ WBS DICTIONARY } \\
\hline \multicolumn{2}{|c|}{$\begin{array}{l}\text { 1 PROJECT TITLE/PARTICIPANT } \\
\text { Spent Nuclear Fuel Project }\end{array}$} & $\begin{array}{l}2 \text { DATE } \\
\text { January } 14,1997\end{array}$ & $\begin{array}{c}3 \text { IDENTIFICATION NO. } \\
14113\end{array}$ \\
\hline \multicolumn{2}{|c|}{4 PROJECT ELEMENT CODE } & \multicolumn{2}{|c|}{5 PROJECT ELEMENT TITLE } \\
\hline 6 INDEX LINE NO. & & $\begin{array}{l}\text { OAND } \\
\text { ION } \\
\quad 0 \\
\end{array}$ & 8 DATE \\
\hline \multicolumn{4}{|c|}{9 APPROVED CHANGES } \\
\hline \multicolumn{2}{|c|}{10 SYSTEM DESIGN DESCRIPTION } & \multicolumn{2}{|c|}{$\begin{array}{c}\text { 11 BUDGET AND REPORTING NUMBER } \\
\text { EW7040000 } \\
\text { EW7002010 (Basin Operations) }\end{array}$} \\
\hline
\end{tabular}

\section{A. STATEMENT OF WORK}

The SNF Operations WBS Level 4 encompasses the work to maintain the facilities in a safe, environmentally sound condition. This includes facility operations and maintenance; handling and storage of spent nuclear fuel; and operational support of the Spent Nuclear Fuel Project.

The scope contained in the WBS element includes the management, technical, clerical and bargaining unit personnel performing maintenance, surveillance, documentation, training, fuel removal operations, procedure preparation, readiness assessments/reviews, and security for the fuel storage basins and the supporting utility systems. Non-labor cost elements include maintenance materials and waste disposal charges.

\subsubsection{OPERATIONS:}




\section{SPENT NUCLEAR FUEL PROJECT \\ WBS 1.4.1}

HNF-SP-1104, Rev 4

\section{KBasin Q \& M:}

Provide for the operation of all K Basin facilities and systems including K East, K West, and the water treatment plant. Provide funds for electric utility power and maintenance assessments, fire systems maintenance assessments, performance of required surveillance, material / waste handling and disposal assessments, emergency preparedness activities, safeguards and security activities, radiological control, safety, and quality assurance.

O\&M provides funding for planning, engineering design/modification support, scheduling, tracking, and performing of maintenance work packages and activities.

O\&M provides funding for development and maintenance of policies and procedures, regulatory compliance and technical safety activities, including engineering design support and configuration control of baseline documentation.

O\&M provides funding for development, implementation, and management of the training program for all O\&M staff, including all required qualifications and certifications.

O\&M provides funding for $K$ Basins independent oversight and self assessment activities, $K$ Basins Management Activities, tracking of commitments and action items as well as management direction and administrative services.

\section{FRS Operations:}

Perform operations planning, mobilization, start-up and ORR of the FRS and Cask/Transportation System. This includes operations staff ramp up and training, establishment of FRS management systems, procedure development, and start-up and operational testing. This activity also provides operational input to the Projects for design, procurement, permitting, and Safety Analysis Report development, as well as operational support for fuel retrieval.

O\&M provides funding for plant operational activities including shift management, facility maintenance, HPT and lab services and surveillance, training, and procedural development.

\section{Debris Removal Operations:}

Includes removal of underwater debris from $105 \mathrm{KE}$ and $105 \mathrm{KW}$ Basins. Efforts will focus on moving and/or removing empty fuel canisters and clearing areas to facilitate fuel and sludge removal from the basins. Operational activities for the removal of the empty canisters, old tools, cables, gloves, and miscellaneous materials of debris in $105 \mathrm{KE}$ and $105 \mathrm{KW}$ Basins are included. Debris Removal activities include removing debris from the South Load Out Pit, cleaning and 


\section{SPENT NUCLEAR FUEL PROJECT \\ WBS 1.4.1}

HNF-SP-1104, Rev 4

removing empty canisters from the basins prior to fuel processing and removal, and general debris removal.

\section{CVD Operations:}

Perform Cold Vacuum System start-up, ORR, and operations. This includes operations staff ramp up and training, establishment of CVD management systems, and procedure development. This activity also provides operational input to the CVD Project for design, procurement, permitting, and Safety Analysis Report development, as well as operational support for the CVD.

\section{Water Treatment Operations:}

The integrated water system is needed to handle particulate and ion loading in basin water due to liberation of canister sludge and basin turbidity. Operational activities for the treatment of water in the basins include replacement of Ion Exchange Modules (IXMs) and Cartridge Filters (Cfs). Disposition of 45 Legacy Water System IXC's, design, installation, safety analysis, preparation for operations and readiness review.

\section{Sludge Retrieval Operations:}

Provides systems to manage the sludge/sediment on the floor and pits of $105 \mathrm{~K}$ East (KE) and the sand filter backwash pit in $105 \mathrm{~K}$ West $(\mathrm{KW})$ Basins, and canister sludge in $\mathrm{KE}$ and $\mathrm{KW}$ and floor sludge in KE. The sub-project will retrieve the sludge from the KE Basin floor and pits and transfer to staging areas with the basin; treat/package the sludge; and transfer the sludge to long term storage outside the K Basins. Sludge removal will be executed in two phases. The first phase consists of retrieving and relocating the KE floor sludge to the weasel pit area for temporary staging. The second phase consists of removing sludge from the KE pits/staging area and the $K W$ sand filter backwash pit to a double shell tank at TWRS. The activity also provides operational input to the Sludge Retrieval Project for design, procurement, permitting and safety analysis report development, as well as operational support for sludge retrieval.

\section{HCS Operations:}

Perform Hot Conditioning System start-up, ORR, and operations. This includes operations staff ramp up and training, establishment of HCS management systems, and procedure development. This activity also provides operational input to the HCS Project for design, procurement, permitting, and Safety Analysis Report development, as well as operational support for the HCS. 


\section{SPENT NUCLEAR FUEL PROJECT \\ WBS 1.4.1}

HNF-SP-1104, Rev 4

\section{CSB Operations:}

Perform CSB start-up, ORR, and operations. This includes operations staff ramp up and training, establishment of CSB management systems, and procedure development. This activity also provides operational input to the CSB Project for design, procurement, permitting, and Safety Analysis Report development, as well as operational support for the CSB.

The Sub-project Phase 1 elements are as follows:

K Basin O\&M

K Basins Baseline Documentation: (1.4.1.03.01.03)

Fuel Relocation/HCS Common Operations (1.4.1.03.01.06)

K Basin Operations: (1.4.1.03.01.01)

Maintain K Basins: (1.4.1.03.01.02)

K Basins Staff Training: (1.4.1.03.01.04)

$\mathrm{K}$ Basins Management (1.4.1.03.01.05)

FRS Operations

FRS K Basin Start-Up/Training (1.4.1.04.03.07)

FRS K Basin Operations (1.4.1.04.03.08)

Testing and ORR of Transportation: (1.4.1.06.01.06)

Debris Removal Operations

Debris Removal Operations (1.4.1.04.05.02)

CVD Operations

CVD Facility Start-Up \& MSA: (1.4.1.08.03.06)

Operations of CVD Facility: (1.4.1.08.04.01,1.4.1.08.04.02, 1.4.1.08.04.06)

Water Treatment Operations

Water Treatment Operations: (1.4.1.04.06.02)

Sludge Removal Operations

K Basin Sludge Removal Operations (1.4.1.04.04.02) 


\section{SPENT NUCLEAR FUEL PROJECT \\ WBS 1.4.1}

HNF-SP-1104, Rev 4

\section{HCS Operations}

HCS Start-Up \& ORR (1.4.1.08.01.06)

Hot Conditioning System Operations (1.4.1.08.02.01, 1.4.1.08.02.02,

1.4.1.08.02.03)

CSB Operations

CSB Start-Up \& MSA: (1.4.1.07.01.05)

Operations of CSB: (1.4.107.02.01, 1.4.1.07.02.02, 1.4.1.07.02.03, $1.4 .1 .07 .02 .04,1.4 .1 .07 .02 .05,1.4 .1 .07 .02 .06)$

Major end-item deliverables (related to milestones and interfaces appropriate to this level of the sub-project):

- Maintain and operate the fuel storage basins at $100 \mathrm{~K}$ Area, CVD, and CSB/HCA in a safe and environmentally sound manner.

- Complete and verify training for special nuclear fuel handling personnel in compliance with DOE order.

- Complete required surveillance and maintenance to ensure compliance with TSR and SAR.

- Complete the management system portion - SNF ORR.

- Train Operators and Complete ORRs.

- Clean canisters.

- Remove debris.

- Remove sludge

- Clean, repackage, load and transport fuel from K Basins to CSB.

- Operate integrated water system to ensure water quality during fuel removal.

- Disposition of 45 Legacy Water System IXC's, design, installation, safety analysis, preparation for operations and readiness review. 


\section{SPENT NUCLEAR FUEL PROJECT \\ WBS 1.4.1}

HNF-SP-1 104, Rev 4

WBS LEVEL 4 DICTIONARY

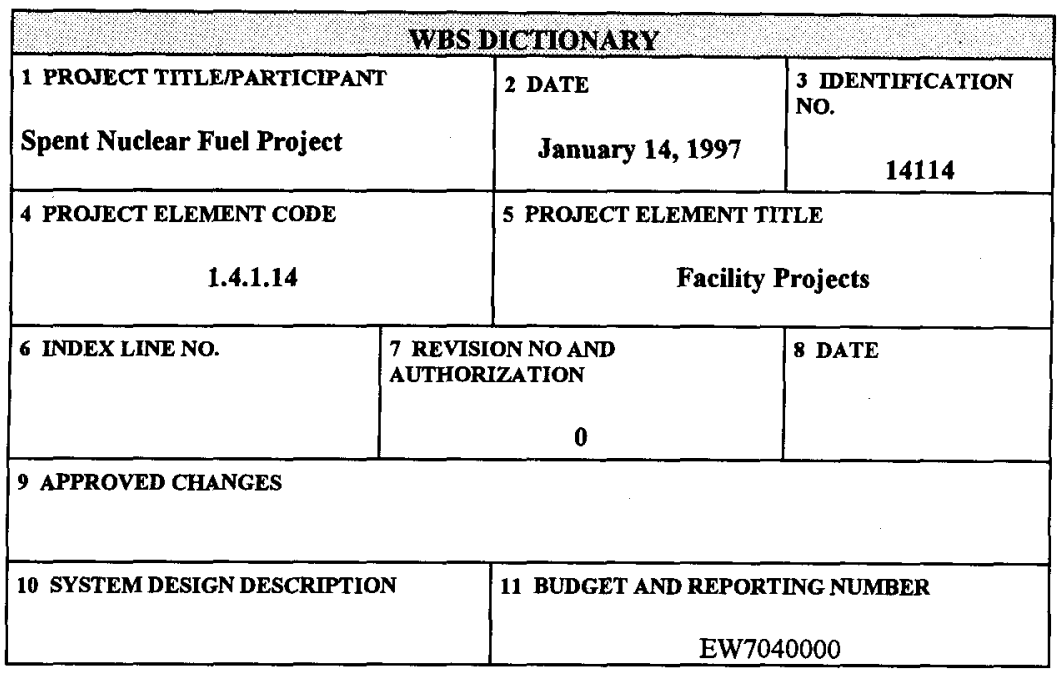

\section{A. STATEMENT OF WORK}

\section{Work To Be Performed}

The Facility Projects WBS element contains sub-projects to upgrade the K Basin Facility for safety and efficiency in supporting the additional personnel and increased activity that are expected during upcoming removal projects, to maintain and operate a Cold Test Facility, to reduce personnel dose in the $\mathrm{K}$ Basins, to remove fuel, sludge, and debris from the $\mathrm{K}$ Basins and to continue basin water treatment throughout the project.

For each sub-project, the work includes the functions and requirements specification, design, procurement, installation, acceptance testing, safety documentation, environmental compliance and permitting. The scope also includes the operations related activities to prepare procedures, train personnel and conduct operational readiness assessments/reviews, the operations cost for transportation of fuel or waste to the destination location, and the cost and interface with Hanford Site waste disposition facilities. 


\section{SPENT NUCLEAR FUEL PROJECT \\ WBS 1.4.1}

HNF-SP-1 104, Rev 4

The sub-projects included are: K Basins Facility Upgrades, Dose Reduction System, Fuel Retrieval, Sludge Removal, and Debris Removal, Integrated Water Treatment, and Deactivation Preparation.

Facility Upgrades - Included in this sub-project are activities to repair, replace and modify essential K Basins infrastructure to facilitate successful execution of Spent Fuel mission objectives, close out the project records for the roof repair activity, installation of MO-442 in 100K (completed FY96), modify and maintain a cold test facility for K Basin fuel, sludge and debris removal and dose reduction equipment development, acceptance testing and operator training, evaluate the needs and construct additional space for change rooms, clothing storage, lunch rooms, rest rooms, and office space to accommodate increased staff, and perform other facility modifications for fuel removal support. Facility Upgrades for Cask Transportation Construction activities associated with preparing the South Transfer Areas in both KE and KW basins for the Cask Transportation System, and installation of same. Initial work will involve removing systems and structures that are no longer in use and upgrading the 30 ton cranes to allow for safe, efficient cask transportation operations. Other construction activities will be to install the immersion pail support structure and the Multi-canister overpack (MCO) loading system.

Dose Reduction System - Includes mitigation of dose from basin walls and mitigation of dose and dose management for $K$ East and $K$ West Basins for all of the basin projects. For basin wall dose mitigation, the basin walls will be cleaned and coated and the basin water level raised in $105 \mathrm{KE}$ Basin (completed FY96). Dose management includes global ALARA planning based on dose assessments to help determine staffing requirements, and dose reduction equipment procurement for individual projects' operations. Additional dose reduction will be required for $105 \mathrm{KE}$ Basin in FY 97 to reduce the dose received by the increased personnel expected in the basins during fuel re-racking. This will include hydrolasing of piping and equipment in high traffic areas, such as the transfer bay and dummy elevator pit, decontaminating of piping and equipment that is slated to be removed, and decontaminating and refinishing the concrete floors in both basins. A PA communication system will be installed in both $\mathrm{KE}$ Basin and KW Basin.

Fuel Retrieval - Acquisition and testing of the equipment to retrieve fuel canisters from storage bays, open (KW only), clean fuel elements to remove all unbonded sludge and corrosion particles and remove stuck fuel elements from canister. Fuel and fuel scrap loading in MCO tier baskets, and transfer of loaded tier baskets to a queue station for lag storage.

Sludge Removal - This sub-project provides systems to manage the sludge/sediment on the floor and in the pits of $105 \mathrm{KE}$ and $105 \mathrm{KW}$ Basins and any sludge/sediment contained in the fuel canisters, to remove/retrieve the sludge, to pre-treat/package the sludge, and to transfer the 


\section{SPENT NUCLEAR FUEL PROJECT \\ WBS 1.4.1}

HNF-SP-1 104, Rev 4

sludge for disposal. Sludge removal will be executed in two phases. The first phase consists of relocating sludge for temporary staging during fuel retrieval. The second phase consists of transferring sludge from staging to double shell tank at TWRS. Retrieval and disposal of KW floor sludge is not included in this scope, anticipating $R L$ approval of the contractor level MOU between SNFP and Transition Projects.

Debris Removal - This sub-project includes removal of underwater debris from $105 \mathrm{KE}$ and 105 KW Basins. Efforts will focus on moving and/or removing empty fuel canisters and clearing areas to facilitate fuel and sludge removal from the basins. Debris Removal activities include removing debris from the South Load Out Pit, cleaning and removing empty canisters from the basins prior to fuel processing and removal, and general debris removal.

Integrated Water Treatment - The Integrated Water Treatment System (IWTS) provides water filtration and treatment necessary to maintain water quality in the basins. It will upgrade and provide new water treatment systems and facilities for both the KE and KW basins. The IWTS will provide clean, treated, or fresh water and receive and treat contaminated water for the fuel, sludge, and debris removal systems, as well as the MCO/Cask system. The IWTS will provide treatment capability to maintain water clarity, remove decay heat, and maintain soluble and insoluble radionuclide concentrations in the basins as low as reasonably achievable during continued SNF storage and fuel and sludge removal actives. The IWTS will also install a system for canister sludge management during fuel removal. This system will put filter backwash material into the Weasel Pit.

The Sub-project Phase 1 elements are as follows:

Facility Upgrades

Essential Systems (1.4.1.04.01.01)

Cold Test Facility (1.4.1.04.01.05)

Basin Personnel Facility Upgrades (1.4.1.04.01.07)

Basin Facility Support Upgrades (1.4.1.04.01.08)

Dose Reduction System (1.4.1.04.02.01)

K Basin Facility Upgrades for Transport (1.4.1.06.01.08)

Fuel Retrieval

FRS Technical Baseline (1,4.1.04.03.01)

FRS Design (1.4.1.04.03.03)

FRS Procurement/Fab/Construction (1.4.1.04.03.04)

FRS Equipment Test (1.4.1.04.03.05)

FRS Regulatory Compliance (1.4.1.04.03.06) 


\section{SPENT NUCLEAR FUEL PROJECT \\ WBS 1.4.1}

HNF-SP-1104, Rev 4

FRS Project Management (1.4.1.04.03.02)

Sludge Removal

Sludge Removal System (1.4.1.04.04.01)

Debris Removal

Debris Removal Project (1.4.1.04.05.01)

Integrated Water System

Water Treatment System (1.4.1.04.06.01)

Deactivation Preparation (1.4.1.04.07.01)

Major end-item deliverables (related to milestones and interfaces appropriate to this level of the sub-project):

- Essential system upgrades to basin. This will include upgrades to the electrical system, maintenance facility and potable water system. Also provides minimal fire protection.

- Relocation of personnel trailers

- $\quad$ K Basins roof repairs (completed FY96)

- Availability of the Cold Test Facility for design development and operator training.

- Design, installation and readiness review of Fuel Removal Support Facilities

- Dose Reduction associated with basin walls and piping. Also will include floor refinishing and superstructure decon.

- Fuel Retrieval System design, installation, safety analysis, preparation for operations, and Operational Readiness Review.

- Sludge Removal System design, installation, safety analysis, preparation for operations, and Operational Readiness Review.

- Debris Removal System design, installation, safety analysis, preparation for operations, and Operational Readiness Review. 


\section{SPENT NUCLEAR FUEL PROJECT \\ WBS 1.4.1}

HNF-SP-1 104, Rev 4

- Water Treatment System design, installation, safety analysis, preparation for operations, and Readiness Assessment.

- K Basin Facilities Turnover agreements/criteria 


\section{SPENT NUCLEAR FUEL PROJECT \\ WBS 1.4.1}

HNF-SP-1 104, Rev 4

WBS LEVEL 4 DICTIONARY

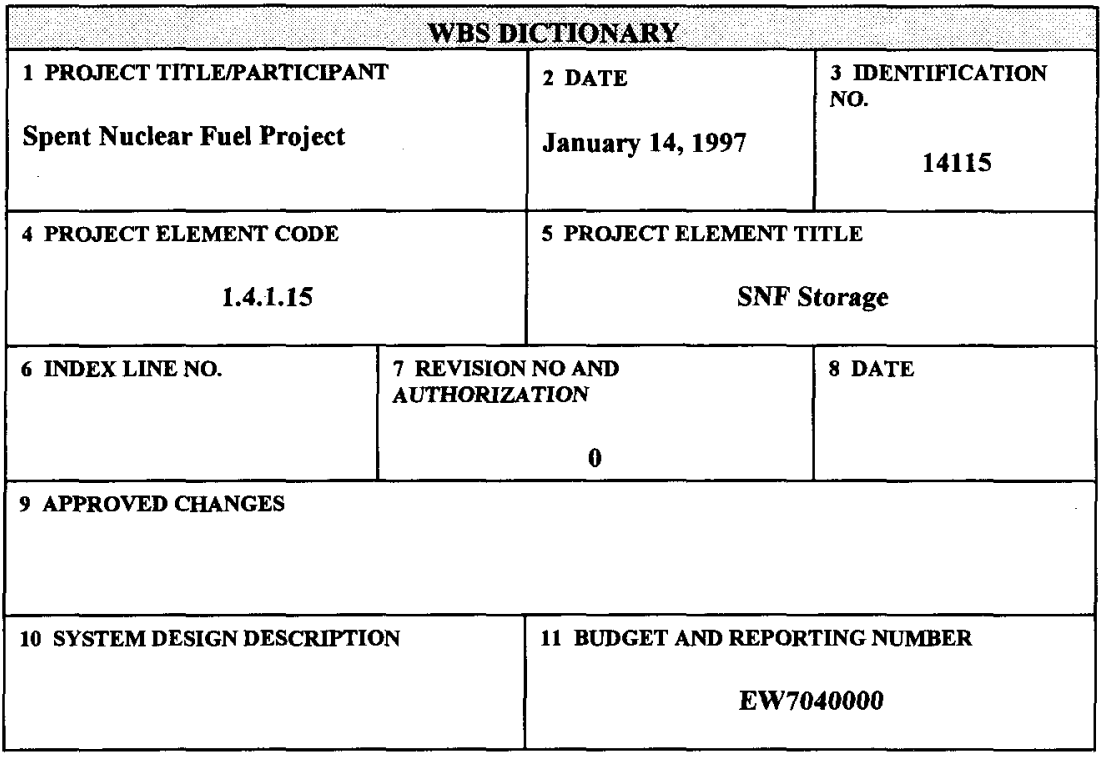

\section{A. STATEMENT OF WORK}

\section{Work To Be Performed}

The SNF Storage WBS Level 4 consists of three sub-projects: Cask/ Transportation System, Multi-Canister Overpack (MCO) Acquisition, and Other Hanford Fuel. Each sub-project is defined below.

Cask Transportation - The Cask/Transportation System encompasses acquisition of the transportation system and transportation casks to transfer the Multi-Canister Overpack (MCO's) from the K Basins to the Cold Vacuum Drying (CVD) station, provides the processing vessel and operating platform for $\mathrm{CVD}$, and then transports the MCO's to the Canister Storage Building (CSB). The transportation system includes the casks, conveyances, ancillary equipment, and an immersion pail system required to preclude contamination of the 


\section{SPENT NUCLEAR FUEL PROJECT \\ WBS 1.4.1}

HNF-SP-1104, Rev 4

cask package. The transportation system also includes acquisition of the MCO loading system which moves the loaded fuel baskets from the basins into the cask loadout and loads the baskets into the Cask/MCO.

The scope includes definition of performance specifications, design and fabrication procurement, safety analyses, qualification testing, acceptance testing, installation verification, and management systems. Also included are interface activities associated with modification of the Basin facilities to accommodate installation and operation of the cask/transportation system. Five Cask/Conveyance systems and two immersion pail systems are required.

The fuel load out and transportation training, readiness assessments/review and operations are contained in other WBS elements. Sludge, debris, and water transportation equipment are also contained in other WBS elements.

Multi-Canister Overpack (MCO) - The Multi-Canister Overpack (MCO) Acquisition encompasses acquisition of containers which will house the spent nuclear fuel during cold vacuum drying, transportation to the Canister Storage Building (CSB), staging at the CSB, hot conditioning, and interim storage at the CSB. $400 \mathrm{MCO}$ 's are estimated to be required. The scope includes the establishment of the MCO performance specification, design, process testing, acceptance testing, fabrication, procurement, safety documentation, and management systems. The MCO includes the shell, closure mechanism, and internal structures (fuel and scrap baskets), and internal provisions to accommodate the drying and conditioning processes.

The facilities, equipment, training, readiness assessment/reviews and operations to load and transport the MCO's are contained in other WBS elements.

Other Hanford Fuel - Other Hanford Fuel encompasses SNF Project activities necessary to attain safe interim storage of the SNF throughout the Hanford Site that is not currently stored at the $\mathrm{K}$ Basins and manage these materials in accordance with the SNF and INEL EIS Record of Decision and DOE/Navy/State of Idaho Consent Order. The SNF inventory in the 300 and 400 areas will be consolidated at the 400 Area Interim Storage Area (ISA), primarily with non-SNF Project funds. The SNF Project will fund certain activities to maintain the materials at the 400 Area ISA in accordance with approved project interface agreements. The 400 Area ISA SNF will later be transferred to a 200 Area ISA, which will include a pad provided under this WBS. T Plant SNF will be transferred to the CSB after emplacement of $K$ Basins SNF at the CSB. Plans will be developed to repackage and interim store PFP and LLBG SNF. Sodium bonded FFTF SNF will be transloaded at the CSB into T-3 casks and transferred to INEL.

The scope includes acquisition of casks, safety analysis, transportation of fuel onsite, interim 


\title{
SPENT NUCLEAR FUEL PROJECT \\ WBS 1.4.1
}

HNF-SP-1104, Rev 4

storage surveillance and security and transportation of the fuel offsite as prescribed by the interprogram MOUs. Work scope and associated costs after the endpoint defined by the Project Management Plan are included for information purposes only.

Other Hanford Fuel includes: Oregon State University reactor TRIGA fuel currently located at the Low Level Waste Burial Grounds; Shippingport PWR Core 2 fuel currently located at T Plant; Light Water Reactor fuel from the PNNL 324, 325, and 327 facilities; TRIGA fuel currently located at the 400 Area/SA (previously at the 308 Facility Annex); FFTF fuel currently located at the FFTF; LAMPRE and University of Washington reactor fuel currently located at the PFP facility; and N Reactor fuel fragments in the N Basins sludge currently located at the $\mathrm{N}$ Basins.

The Sub-project Phase 1 elements are as follows:

\author{
MCO Acquisition \\ MCO Acquisition Definition (1.4.1.05.01.01) \\ MCO Project Management (1.4.1.05.01.02) \\ Testing/Qualification MCO's (1.4.1.05.01.05) \\ MCO Design (1.4.1.05.01.03) \\ MCO Fabrication (1.4.1.05.01.04) \\ MCO Topical Safety Report (1.4.1.05.01.06) \\ Cask/Transportation \\ Cask/Transportation Project Management (1.4.1.06.01.02) \\ Cask \& Transportation System Design (1.4.1.06.01.03) \\ Cask \& Transportation System Fabrication (1.4.1.06.01.04) \\ Cask/Transportation Regulatory Compliance (1.4.1.06.01.07) \\ Other Hanford Fuel \\ FFTF (1.4.1.09.01.06) \\ PNNL Labs, Buildings 324, 325, 327 (1.4.1.09.01.04) \\ 308 Annex (1.4.1.09.01.05)
}


PFP (1.4.1.09.01.07)

T Plant (1.4.1.09.01.03)

National Programs (1.4.1.01.01.07)

Major end-item deliverables (related to milestones and interfaces appropriate to this level of the sub-project):

Multi-Canister Overpack (MCO):

- Approve MCO Fabrication

- $\quad$ Approve MCO Topical Safety Report

- Issue MCO Design Review Report

- $\quad$ Critical Decision 3 for MCO fabrication

- Award MCO Fabrication Contract

- Receive First MCO

- Complete Fabrication and Delivery of MCOs

Cask/Transportation:

- Award Cask/Transport System Design and Fabrication Contract

- Complete Cask/Transportation Performance Testing

- Complete Cask/Transport System Design

- Complete/Issue Cask/Transportation SARP

- Critical Decision 3 for Cask Fabrication

- Complete Cask/Transportation System Fabrication

- Receive Cask/Transport Systems for Training

- Complete Cask/Operations Equipment Acceptance Test

- Complete MCO Loading System Acceptance Test

Other Hanford Fuel:

- The transfer of spent nuclear fuel located at T Plant, PNNL Labs, FFTF, and the 400 Area ISA to an interim storage pad located adjacent to the CSB in the 200 Area (or to the CSB in the case of SNF at T Plant)

- Operations and surveillance of the LWR and TRIGA SNF at the 400 Area

- $\quad$ Acquiring canisters and drying system for implementing dry storage of T Plant SNF

- Activities to investigate repackaging capability at the CSB for small SNF packages, until the SNF project is transferred to the successor organization

- Acquisition of sodium bonded FFTF transloading capability and associated SNF transfer to INEL 


\section{WBS LEVEL 4 DICTIONARY}

\begin{tabular}{|c|c|c|}
\hline \multicolumn{3}{|c|}{ WBS DICIONANA } \\
\hline $\begin{array}{l}\text { I PROJECT TITLE/PARTICIPANT } \\
\text { Spent Nuclear Fuel Project }\end{array}$ & $\begin{array}{l}2 \text { DATE } \\
\text { January 14, } 1997\end{array}$ & $\begin{array}{l}3 \text { IDENTIFICATION } \\
\text { NO. } \\
\qquad 14116\end{array}$ \\
\hline $\begin{array}{c}4 \text { PROJECT ELEMENT CODE } \\
1.4 .1 .16\end{array}$ & \multicolumn{2}{|c|}{$\begin{array}{l}5 \text { PROJECT ELEMENT TITLE } \\
\text { Canister Storage Building }\end{array}$} \\
\hline 6 INDEX LINE NO. & $\begin{array}{c}7 \text { REVISION NO AND } \\
\text { AUTHORIZATION } \\
0\end{array}$ & 8 DATE \\
\hline \multicolumn{3}{|l|}{9 APPROVED CHANGES } \\
\hline 10 SYSTEM DESIGN DESCRIPTION & \multicolumn{2}{|c|}{$\begin{array}{l}11 \text { BUDGET AND REPORTING NUMBER } \\
\text { EW7040000 } \\
\text { 39EW70400 }\end{array}$} \\
\hline
\end{tabular}

\section{A. STATEMENT OF WORK}

\section{Work To Be Performed}

The Canister Storage Building (CSB) WBS Level 4 encompasses the acquisition to stage and store the spent nuclear fuel after removal from the basins. Scope of the WBS element also includes construction of the annex which will house the Hot Conditioning System (design of the annex is within Hot Conditioning WBS element). The facility is sized to storage 2,100 metric tons of fuel in a secure environment. The fuel is to be accommodated within one of three vaults to be constructed. The second and third vaults are to be partially prepared for optional storage of TWRS glass canisters. SNF will be loaded into MCO's at the $105 \mathrm{~K}$ basins and transported to the CSB for staging after cold vacuum drying. The CSB provides staging of the fuel prior to hot conditioning at a co-located conditioning system. Following Hot Conditioning fuel will be returned to the CSB storage tubes where it will be placed, via a 


\section{SPENT NUCLEAR FUEL PROJECT \\ WBS 1.4.1}

HNF-SP-1104, Rev 4

MCO Handling Machine (MHM), in storage. The facility has a design life of 40 years and will be designed with features to facilitate future life extension to 75 years with necessary refit and future upgrade of the CSB facility.

Under the mission of the SNF Project, the operation of the CSB is transferred to a facilities maintenance program in September 2001. Deactivation of the CSB is budgeted for, but will not be performed by the SNF Project.

Acquisition includes the definition of functions and requirements, design, procurement, construction, safety documentation, environmental compliance and permitting.

Project management includes, cost and schedule reporting, change control, administrative systems tasks, key decision process, ICE reviews, project validation, request for proposal, statement of work for design, construction, startup, and Quality Assurance program. Review and approve contractor submittals.

Prepare engineering trade studies in support of conceptual design and advanced conceptual design. Provide the Definitive Design for the CSB, including system and discipline drawings, vendor information, procurement specification, design calculations, design verification documents, and test procedures. Provide Title III Engineering and Inspection activities to assure that the project is constructed in accordance with the plans and specifications and that the quality of materials and workmanship is consistent with the requirements of the project.

Perform construction management, construction procurement, and construction of the CSB. The Fixed Price contractor shall build, inspect, and test the CSB based on the approved drawings and specifications supplied by the A-E.

Perform activities related to Environmental Documentation, Regulatory Compliance, and permitting. Activities include obtaining air and water permits, prepare NOC for $\mathrm{DOH}$, and EPA approval. Development of the S/RIDs and the preparation of the Safety Analysis Report. Provide systematic identification of hazards with the CSB. Describe and analyze the adequacy of measures taken to eliminate, control, or mitigate identified hazards, analyze potential accidents and the associated risks.

Acquisition of the MCO handling machine to handle the MCOs from the transportation cask unload station to the storage tubes and Hot Conditioning System. This includes a gantry type crane with a cask, hoisting equipment and controls, and shield gate. This WBS item includes a Value Engineering review, proposal evaluation, design and fabrication support, and the design, fabrication and installation of the equipment. 


\section{SPENT NUCLEAR FUEL PROJECT WBS 1.4.1}

HNF-SP-1104, Rev 4

The Sub-Project Phase 1 elements are as follows:

Canister Storage Building

CSB Acquisition Definition (1.4.1.07.01.01)

CSB Project Management (1.4.1.07.01.02)

CSB Design (1.4.1.07.01.03)

CSB Construction (1.4.1.07.01.04)

MHM Fabrication (1.4.1.07.01.07)

CSB Regulatory Compliance (1.4.1.07.01.06)

Major end-item deliverables (related to milestones and interfaces appropriate to this level of the Sub-Project):

- Award Design Contract

- Definitive Design Report

- Safety Analysis Report

- Key Decisions 0, 1, 2, 3A (award construction, 3B (initiate construction)

- Critical Decisions 3 (Deck), 3A (Superstructure), and 3B (Systems), CD4

- Construction Acceptance of Facility 
WBS 1.4.1

HNF-SP-1104, Rev 4

WBS LEVEL 4 DICTIONARY

\begin{tabular}{|c|c|c|c|}
\hline \multicolumn{4}{|c|}{ WBS DICMONARY } \\
\hline \multicolumn{2}{|c|}{$\begin{array}{l}1 \text { PROJECT TITLE/PARTICIPANT } \\
\text { Spent Nuclear Fuel Project }\end{array}$} & $\begin{array}{l}2 \text { DATE } \\
\text { January 14, } 1997\end{array}$ & $\begin{array}{l}3 \text { IDENTIFICATION } \\
\text { NO. } \\
\qquad 14117\end{array}$ \\
\hline \multicolumn{2}{|c|}{$\begin{array}{l}4 \text { PROJECT ELEMENT CODE } \\
\qquad 1.4 .1 .17\end{array}$} & \multicolumn{2}{|c|}{$\begin{array}{l}5 \text { PROJECT ELEMENT TITLE } \\
\text { Conditioning Projects }\end{array}$} \\
\hline 6 INDEX LINE NO. & & $\begin{array}{l}\text { NO AND } \\
\text { TTION } \\
0\end{array}$ & 8 DATE \\
\hline \multicolumn{4}{|c|}{9 APPROVED CHANGES } \\
\hline \multicolumn{2}{|c|}{10 SYSTEM DESIGN DESCRIPTION } & \multicolumn{2}{|c|}{$\begin{array}{c}11 \text { BUDGET AND REPORTING NUMBER } \\
\text { EW7040000 } \\
\text { 39EW70400 } \\
\end{array}$} \\
\hline
\end{tabular}

\section{A. STATEMENT OF WORK}

\section{Work To Be Performed}

The Conditioning Acquisition will provide equipment to dry and condition spent nuclear fuel stored at K Basins. Conditioning the fuel will occur in two process steps, Cold Vacuum Drying (CVD) and Hot Conditioning (HC).

CVD will be performed in the $100 \mathrm{~K}$ area near the $\mathrm{K}$ Basins and is required prior to both transportation to and staging in the Canister Storage Building (CSB). The process removes bulk water from the MCO by draining and then vacuum drying the contents. Removal of bulk water mitigates fuel corrosion and the associated production of hydrogen gas. This allows the MCO to be transferred to the CSB and staged while minimizing the threat of overheating or over pressurization. 


\section{SPENT NUCLEAR FUEL PROJECT \\ WBS 1.4.1}

HNF-SP-1 104, Rev 4

Hot Conditioning will be performed in an annex to the CSB and will prepare the fuel for interim dry storage. The construction of the annex includes the concrete slab and hot conditioning process pits, the superstructure, electrical power and lighting, and HVAC for the annex area. Annex construction is part of the CSB scope of work and is not included in this WBS. Included in this WBS is the development of the HC process, the design, procurement and installation of the HC process equipment, and the Quality Assurance, Safety Analysis and project management functions associated with it.

The HC process removes chemically bound water (hydrates) and uranium hydrides by applying a combination of heat $(300 \mathrm{C})$ and reduced pressure $(1$ torr). This minimizes the potential for pressurizing the $\mathrm{MCO}$ by removing the constituents that can change phase into a gaseous state. A partial oxidation step creates an oxide layer on the exposed uranium metal surfaces to reduce the reactivity of the fuel in the presence of air or other oxidants.

Hot Conditioning System Acquisition Definition includes the development and maintenance of the baseline documents including the Functions and Requirements and design specifications. It also includes the performance of trade studies, discipline reviews, the preparation and maintenance of interface documentation and the development of product and feed criteria.

Project management for CVD and $\mathrm{HC}$ includes cost, schedule and technical baseline control and reporting, change control, administrative systems tasks, critical decision process, definitive design, construction, and quality assurance.

The Design covers the development of the system and discipline drawings, vendot information, procurement specifications, design calculations, design verification documents and test procedures. It also includes prototype development and prototype testing.

Construction includes construction management, procurement, construction, equipment installation, and title III engineering.

Regulatory Compliance includes the development of the Safety Analysis Report, the Hazards Analysis, air permits and S/RIDs.

The Sub-Project Phase 1 elements are as follows:

Cold Vacuum Drying

Cold Vacuum Drying System Project Management (1.4.1.08.03.03)

Cold Vacuum Drying System Design (1.4.1.08.03.04)

Cold Vacuum Drying System Construction (1.4.1.08.03.05)

Cold Vacuum Drying System Regulatory Compliance (1.4.1.08.03.07) 


\title{
SPENT NUCLEAR FUEL PROJECT \\ WBS 1.4.1
}

HNF-SP-1 104, Rev 4

\author{
Hot Conditioning System \\ Hot Conditioning System Acquisition Definition (1.4.1.08.01.01) \\ Hot Conditioning System Project Management (1.4.1.08.01.03) \\ Hot Conditioning System Design (1.4.1.08.01.04) \\ Hot Conditioning System Construction (1.4.1.08.01.05) \\ Hot Conditioning System Regulatory Compliance (1.4.1.08.01.07)
}

\begin{abstract}
Major end-item deliverables (related te milestones and interfaces appropriate to this level of the Sub-Project):
\end{abstract}

Major end-item deliverables for both Cold Vacuum Drying System and Hot Conditioning System Sub-Projects include:
- Award the Design Contract
- Complete Conceptual Design Report
- Complete Definitive Design Report
- Prepare Safety Analysis Report
- Complete Construction Acceptance of Facility 


\section{SPENT NUCLEAR FUEL PROJECT WBS 1.4.1}

HNF-SP-1104, Rev 4

This page intentionally left blank. 


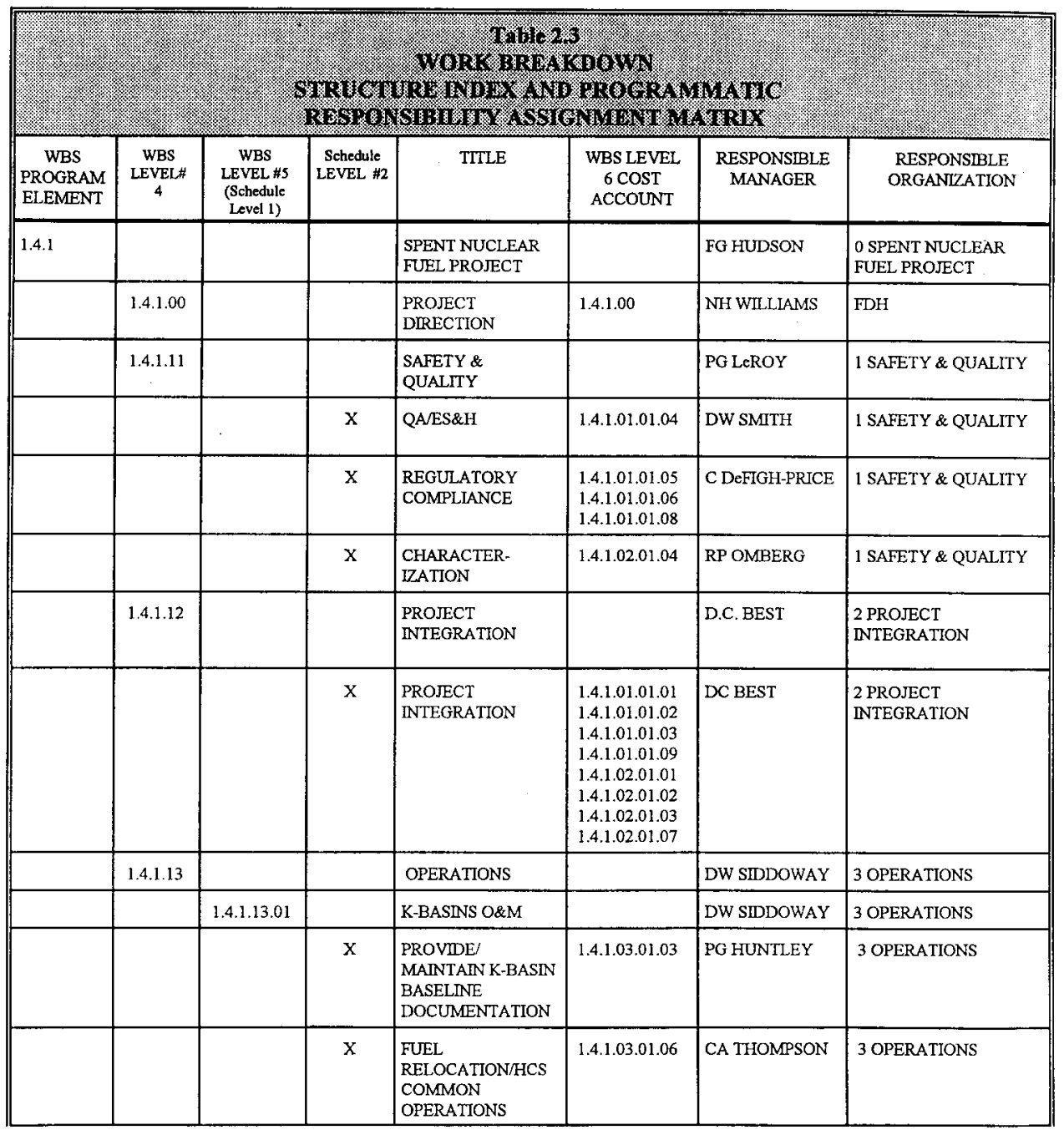




\section{SPENT NUCLEAR FUEL PROJECT \\ WBS 1.4.1}

HNF-SP-1104, Rev :

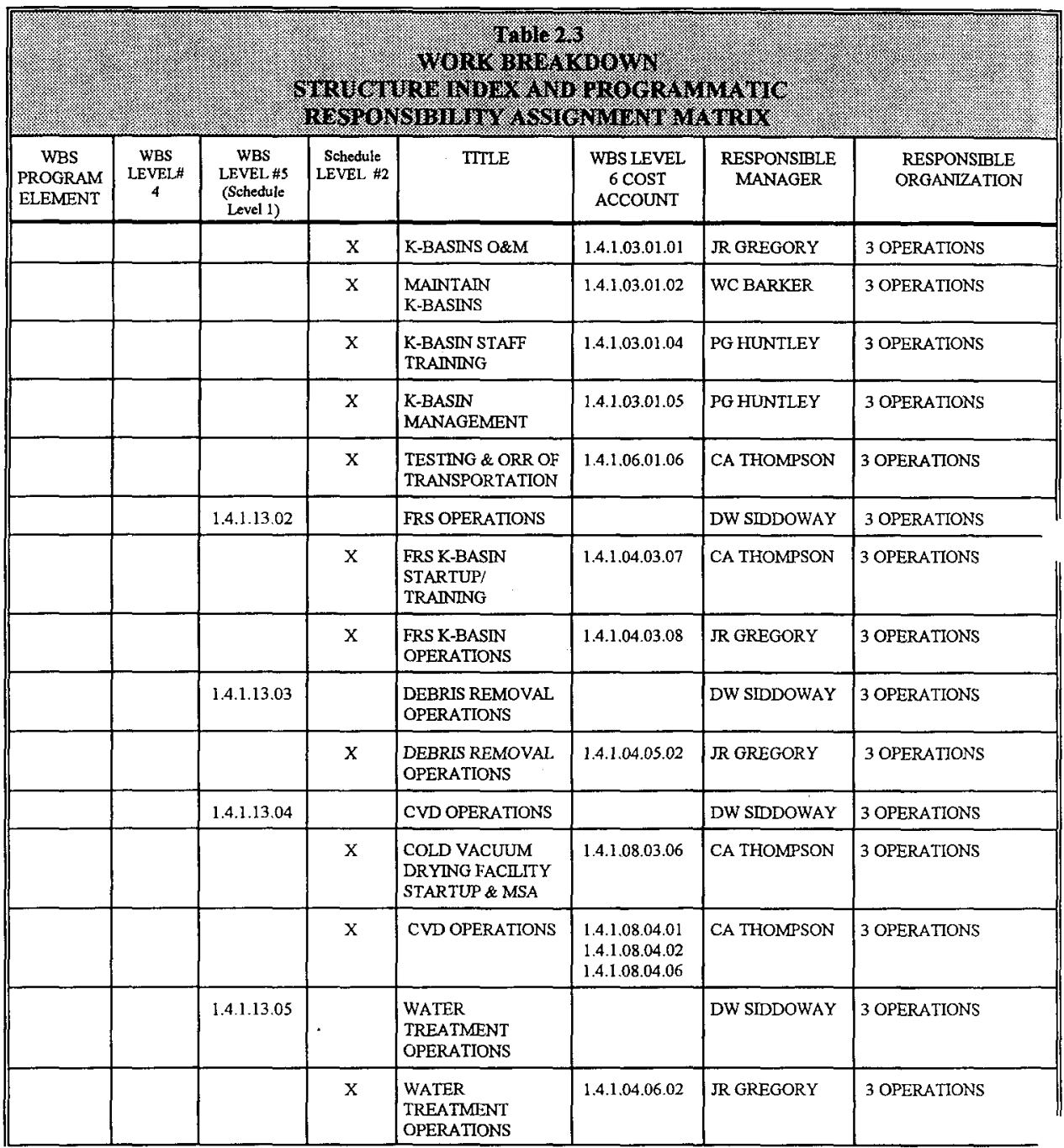




\section{SPENT NUCLEAR FUEL PROJECT \\ WBS 1.4.1}

HNF-SP-1 104, Rev 4

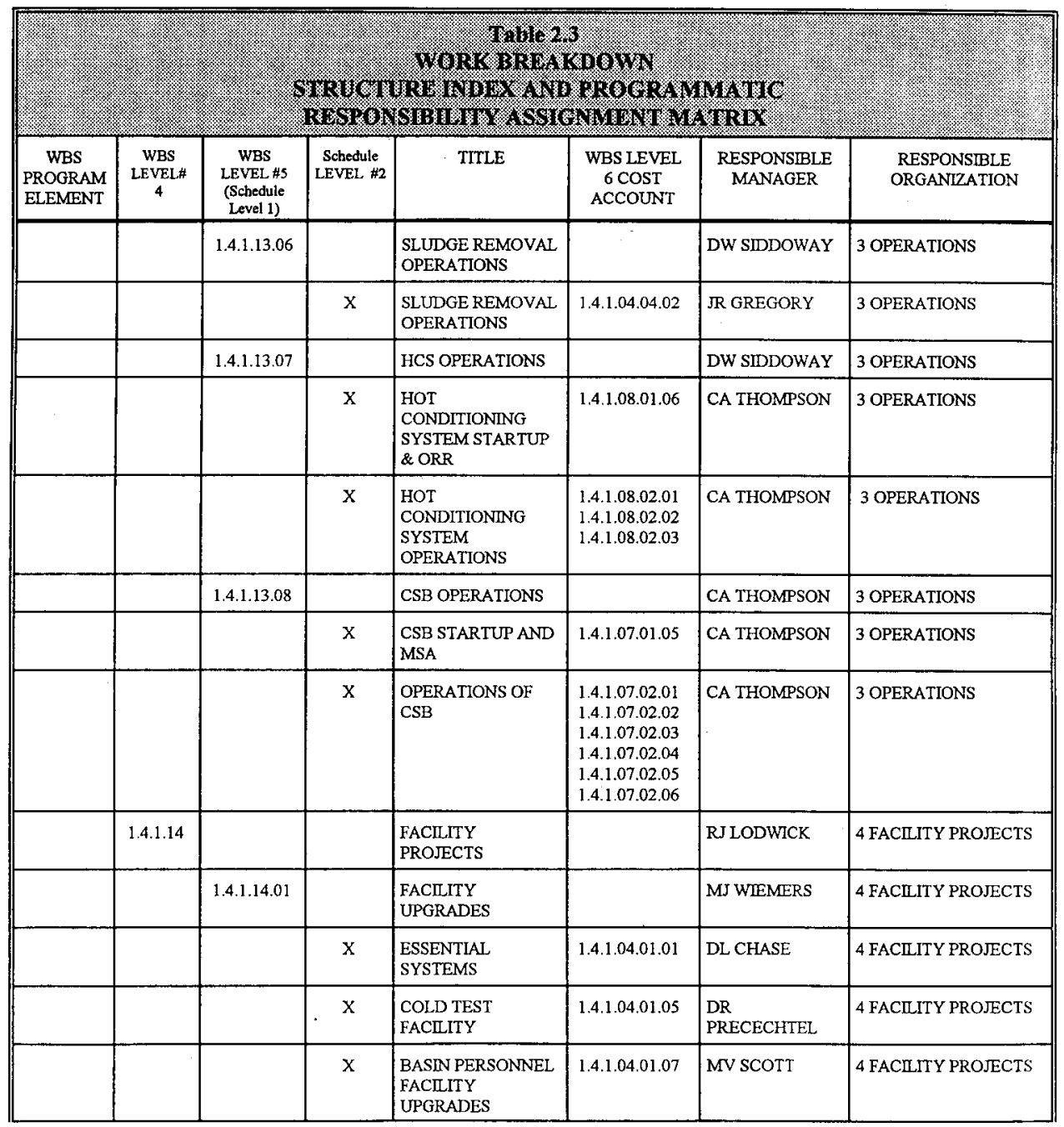




\section{SPENT NUCLEAR FUEL PROJECT \\ WBS 1.4.1}

HNF-SP-1 104, Rev

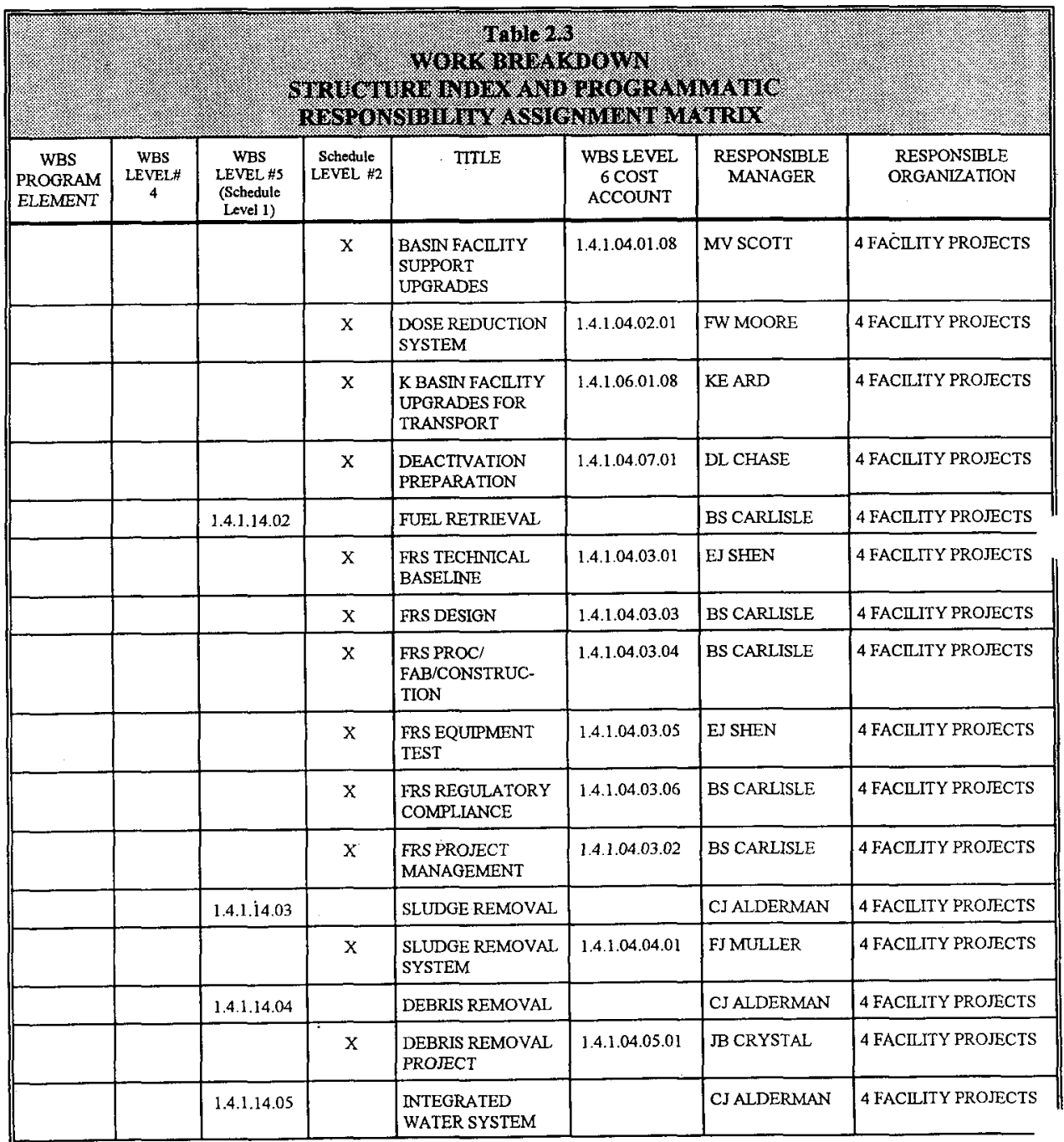




\begin{tabular}{|c|c|c|c|c|c|c|c|}
\hline & & & 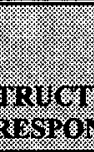 & 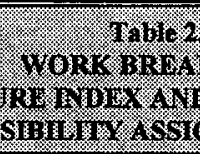 & 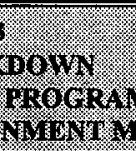 & 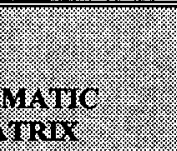 & r. \\
\hline $\begin{array}{c}\text { WBS } \\
\text { PROGRAM } \\
\text { ELEMENT }\end{array}$ & $\begin{array}{l}\text { WBS } \\
\text { LEVEL\# } \\
4\end{array}$ & $\begin{array}{c}\text { WBS } \\
\text { LEVEL \#5 } \\
\text { (Schedule } \\
\text { Level 1) }\end{array}$ & $\begin{array}{l}\text { Schedule } \\
\text { LEVEL \#2 }\end{array}$ & TITLE & $\begin{array}{l}\text { WBS LEVEL } \\
6 \text { COST } \\
\text { ACCOUNT }\end{array}$ & $\begin{array}{l}\text { RESPONSIBLE } \\
\text { MANAGER }\end{array}$ & $\begin{array}{l}\text { RESPONSIBLE } \\
\text { ORGANIZATION }\end{array}$ \\
\hline & & & $\mathrm{x}$ & $\begin{array}{l}\text { WATER } \\
\text { TREATMENT } \\
\text { SYSTEM }\end{array}$ & 1.4.1.04.06.01 & DS TAKASUMI & 4 FACILITY PROJECTS \\
\hline & 1.4.1.15 & & & SNE STORAGE & & $\begin{array}{l}\text { RW } \\
\text { RASMUSSEN }\end{array}$ & 5 SNF STORAGE \\
\hline & & 1.4 .1 .15 .01 & & $\mathrm{MCO}$ & & $\begin{array}{l}\text { RW } \\
\text { RASMUSSEN }\end{array}$ & 5 SNF STORAGE \\
\hline & & & $\mathrm{x}$ & $\begin{array}{l}\text { MCO ACQUISITION } \\
\text { DEFINITION }\end{array}$ & 1.4.1.05.01.01 & KE SMITH & 5 SNF STORAGE \\
\hline & & & $\mathrm{x}$ & $\begin{array}{l}\text { MCO PROJECT } \\
\text { MANAGEMENT }\end{array}$ & 1.4 .1 .05 .01 .02 & KE SMITH & 5 SNF STORAGE \\
\hline & & & $\mathrm{x}$ & $\begin{array}{l}\text { TESTING/ } \\
\text { QUALIFICATION } \\
\text { MCOS }\end{array}$ & 1.4.1.05.01.05 & KE SMTTH & 5 SNF STORAGE \\
\hline & & & $\mathrm{x}$ & MCO DESIGN & 1.4 .1 .05 .01 .03 & KE SMITH & 5 SNF STORAGE \\
\hline & & & $\mathrm{x}$ & MCO FABRICATION & 1.4.1.05.01.04 & KE SMITH & 5 SNF STORAGE \\
\hline & & & $\mathrm{x}$ & $\begin{array}{l}\text { MCO TOPICAL } \\
\text { SAFETY REPORT }\end{array}$ & 1.4.1.05.01.06 & C DEFIGH-PRICE & 5 SNF STORAGE \\
\hline & & 1.4.1.15.02 & & $\begin{array}{l}\text { CASK/TRANSPORT } \\
\text { ATION }\end{array}$ & & $\begin{array}{l}\text { RW } \\
\text { RASMUSSEN }\end{array}$ & 5 SNF STORAGE \\
\hline & & & $\mathrm{x}$ & $\begin{array}{l}\text { CASK/TRANS } \\
\text { PROJECT } \\
\text { MANAGEMENT }\end{array}$ & 1.4 .1 .06 .01 .02 & $\begin{array}{l}\text { RW } \\
\text { RASMUSSEN }\end{array}$ & 5 SNF STORAGE \\
\hline & & . & $\mathrm{x}$ & $\begin{array}{l}\text { CASK/TRANS } \\
\text { DESIGN }\end{array}$ & 1.4 .1 .06 .01 .03 & $\begin{array}{l}\text { RW } \\
\text { RASMUSSEN }\end{array}$ & 5 SNF STORAGE \\
\hline & & & $x$ & $\begin{array}{l}\text { CASK/TRANS } \\
\text { SYSTEM } \\
\text { FABRICATION }\end{array}$ & 1.4 .1 .06 .01 .04 & $\begin{array}{l}\text { RW } \\
\text { RASMUSSEN }\end{array}$ & 5 SNF STORAGE \\
\hline & & & $\mathrm{x}$ & $\begin{array}{l}\text { CASK/TRANS } \\
\text { REGULATORY } \\
\text { COMPLIANCE }\end{array}$ & 1.4.1.06.01.07 & C DEFIGH-PRICE & 5 SNF STORAGE \\
\hline & & 1.4.1.15.03 & & $\begin{array}{l}\text { OTHER HANFORD } \\
\text { FUEL }\end{array}$ & & $\begin{array}{l}\text { RW } \\
\text { RASMUSSEN }\end{array}$ & 5 SNF STORAGE \\
\hline
\end{tabular}




\section{SPENT NUCLEAR FUEL PROJECT \\ WBS 1.4.1}

HNF-SP-1 104, Rev

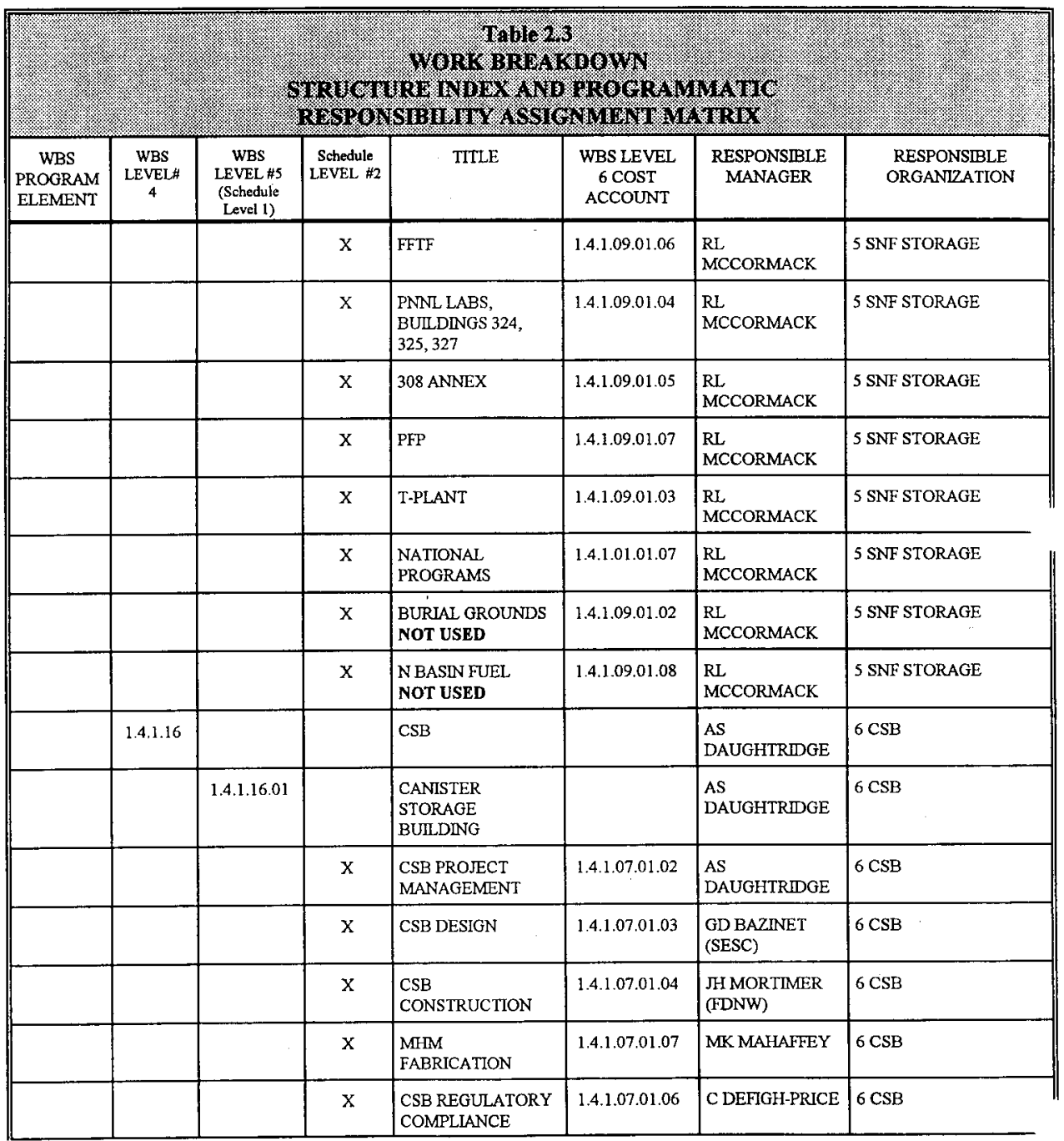




\section{SPENT NUCLEAR FUEL PROJECT \\ WBS 1.4.1}

HNF-SP-1 104, Rev 4

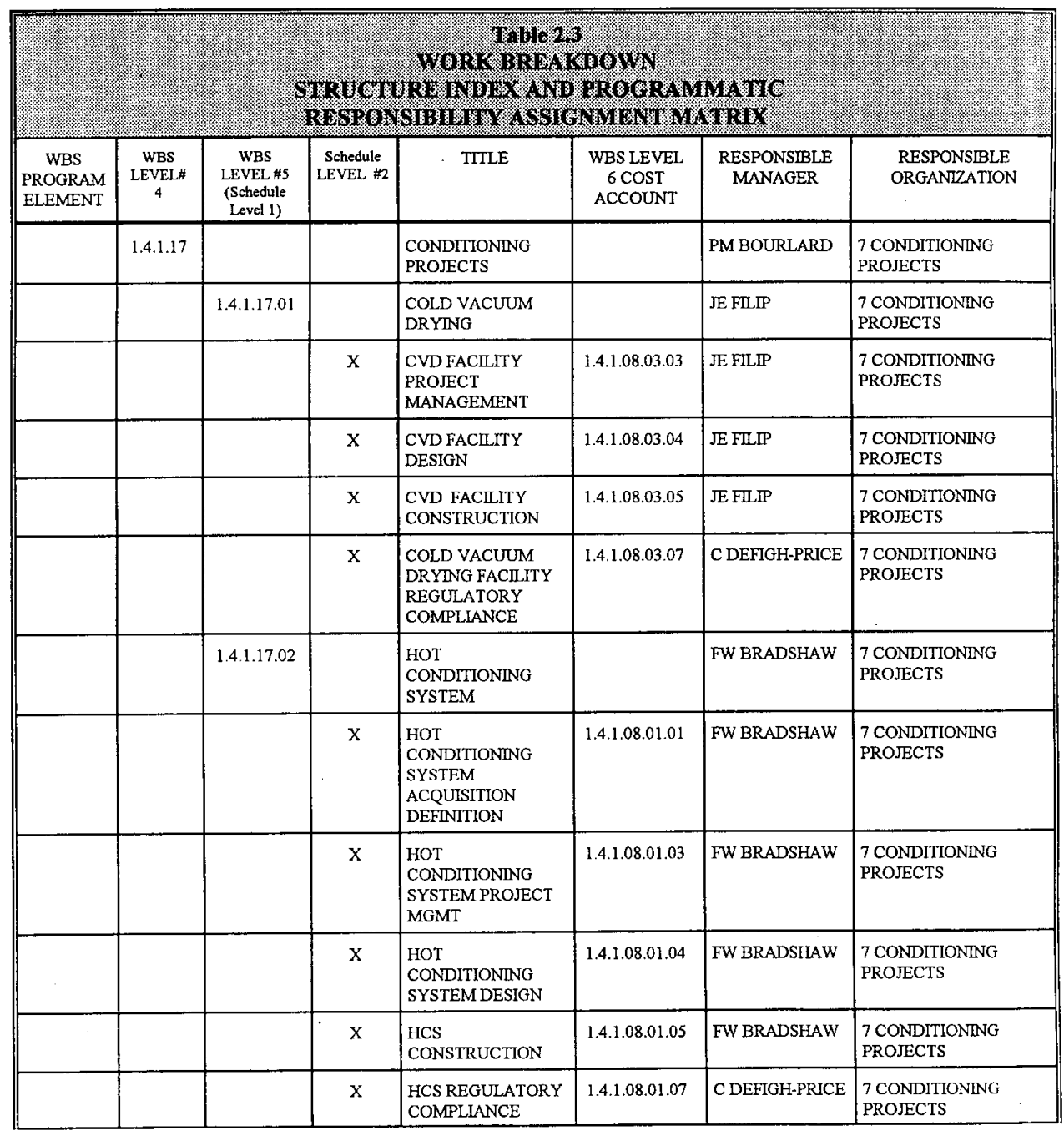




\section{SPENT NUCLEAR FUEL PROJECT \\ WBS 1.4.1}

HNF-SP-1 104, Rev .

\begin{tabular}{|c|c|c|c|c|c|c|c|}
\hline & & & 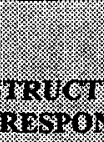 & 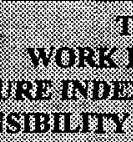 & 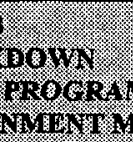 & 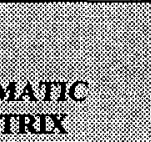 & \\
\hline \multirow[t]{2}{*}{$\begin{array}{c}\text { WBS } \\
\text { PROGRAM } \\
\text { ELEMENT }\end{array}$} & $\begin{array}{c}\text { WBS } \\
\text { LEVEL\# } \\
4\end{array}$ & $\begin{array}{c}\text { WBS } \\
\text { LEVEL \#5 } \\
\text { (Schedute } \\
\text { Level 1) }\end{array}$ & $\begin{array}{c}\text { Schedule } \\
\text { LEVEL \#2 }\end{array}$ & TITLE & $\begin{array}{l}\text { WBS LEVEL } \\
6 \text { COST } \\
\text { ACCOUNT }\end{array}$ & $\begin{array}{l}\text { RESPONSIBLE } \\
\text { MANAGER }\end{array}$ & $\begin{array}{l}\text { RESPONSIBLE } \\
\text { ORGANIZATION }\end{array}$ \\
\hline & & & & NOT USED & $\begin{array}{l}1.4 .1 .02 .01 .05 \\
1.4 .1 .04 .01 .06 \\
1.4 .1 .08 .01 .02 \\
1.4 .1 .08 .03 .02\end{array}$ & & \\
\hline
\end{tabular}


Program Master Baseline Schedule

This page left intentionally blank.

\section{$3.1-1$}


This page intentionally left blank. 


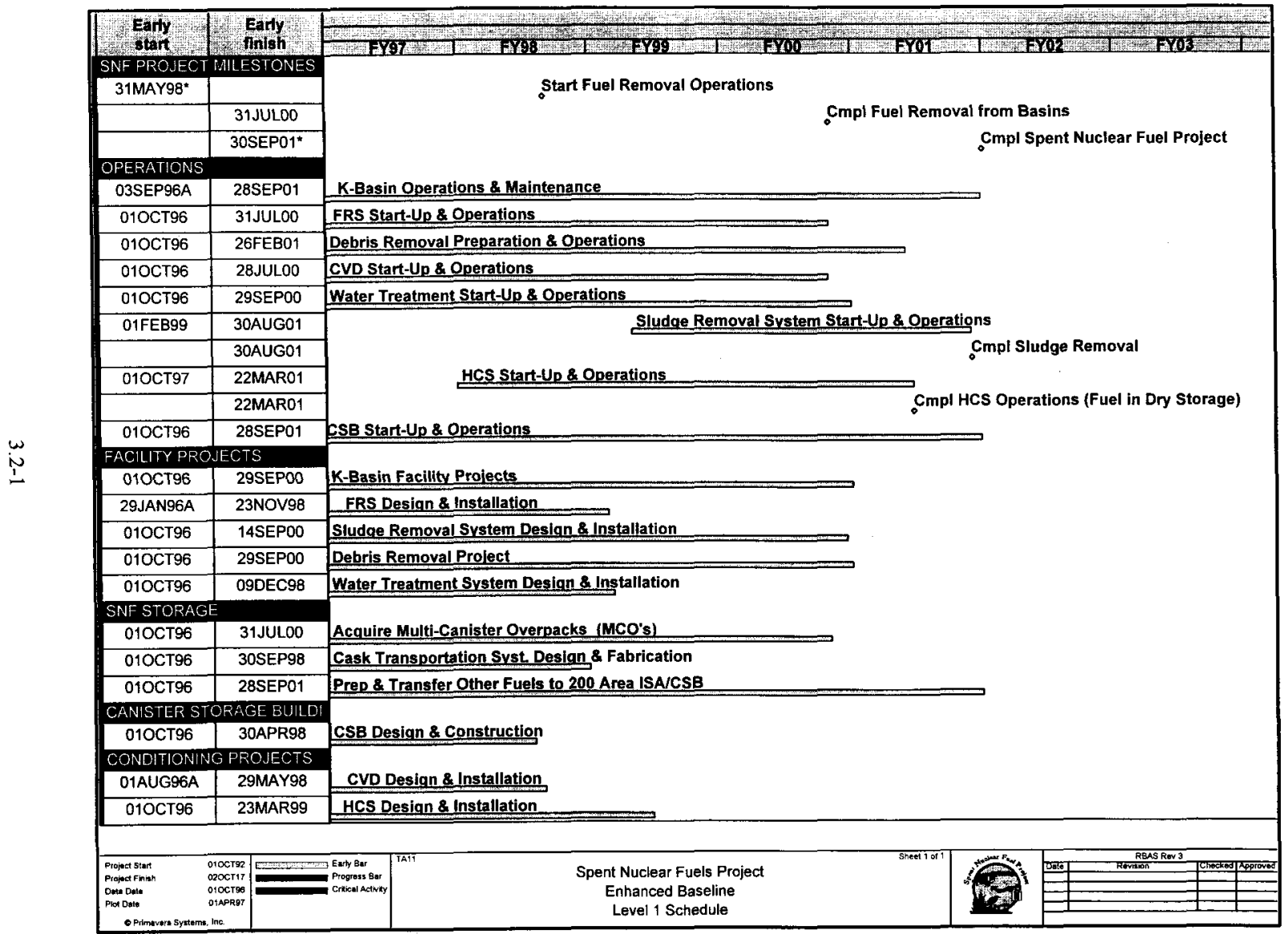




\section{SPENT NUCLEAR FUEL PROJECT}

WBS 1.4.1

HNF-SP-1104, Rev 4

This page intentionally left blank. 
HNF-SP-1 104, Rev 4

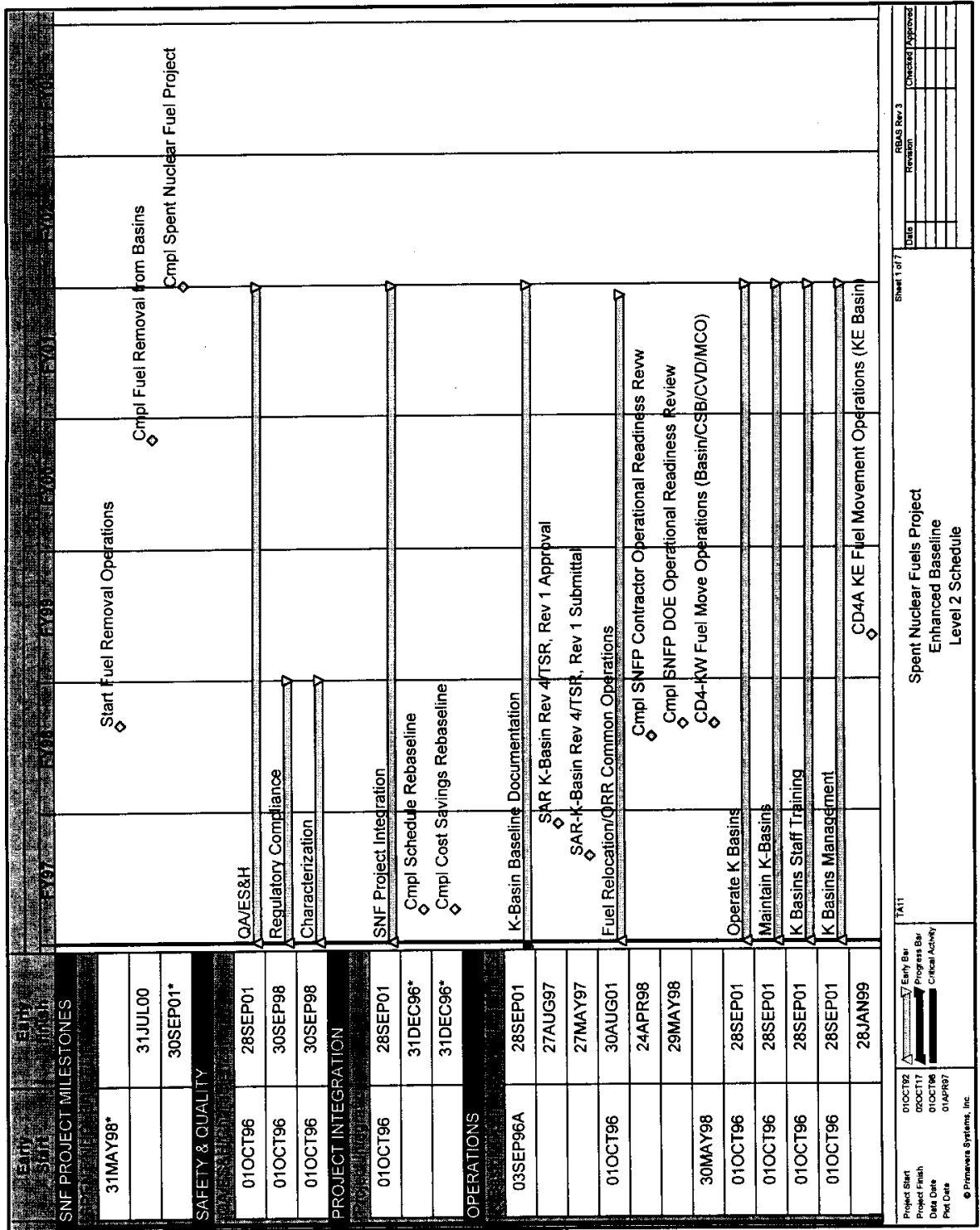




\section{WBS 1.4.1}

HNF-SP-1104, Rev 4

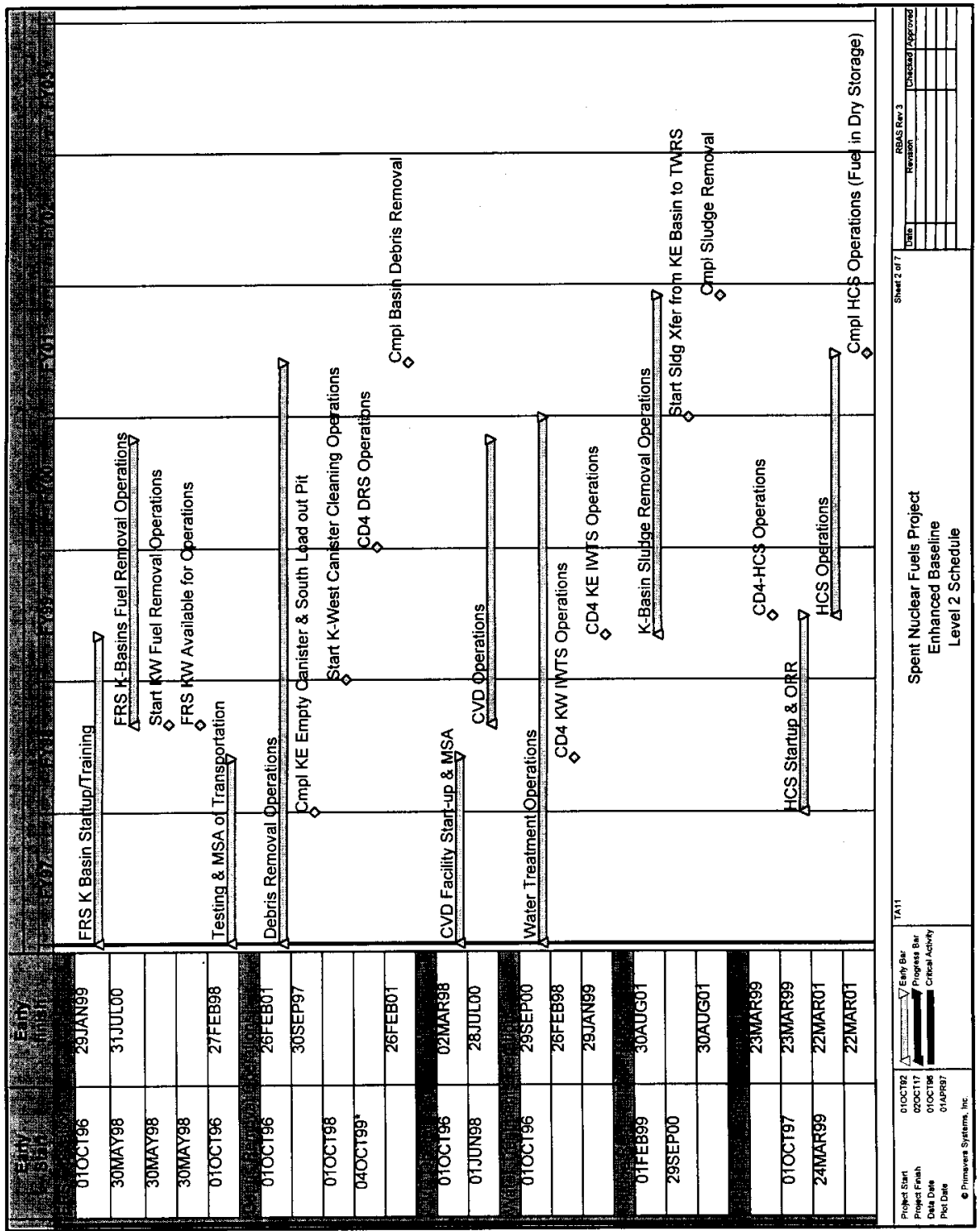


HNF-SP-1104, Rev 4

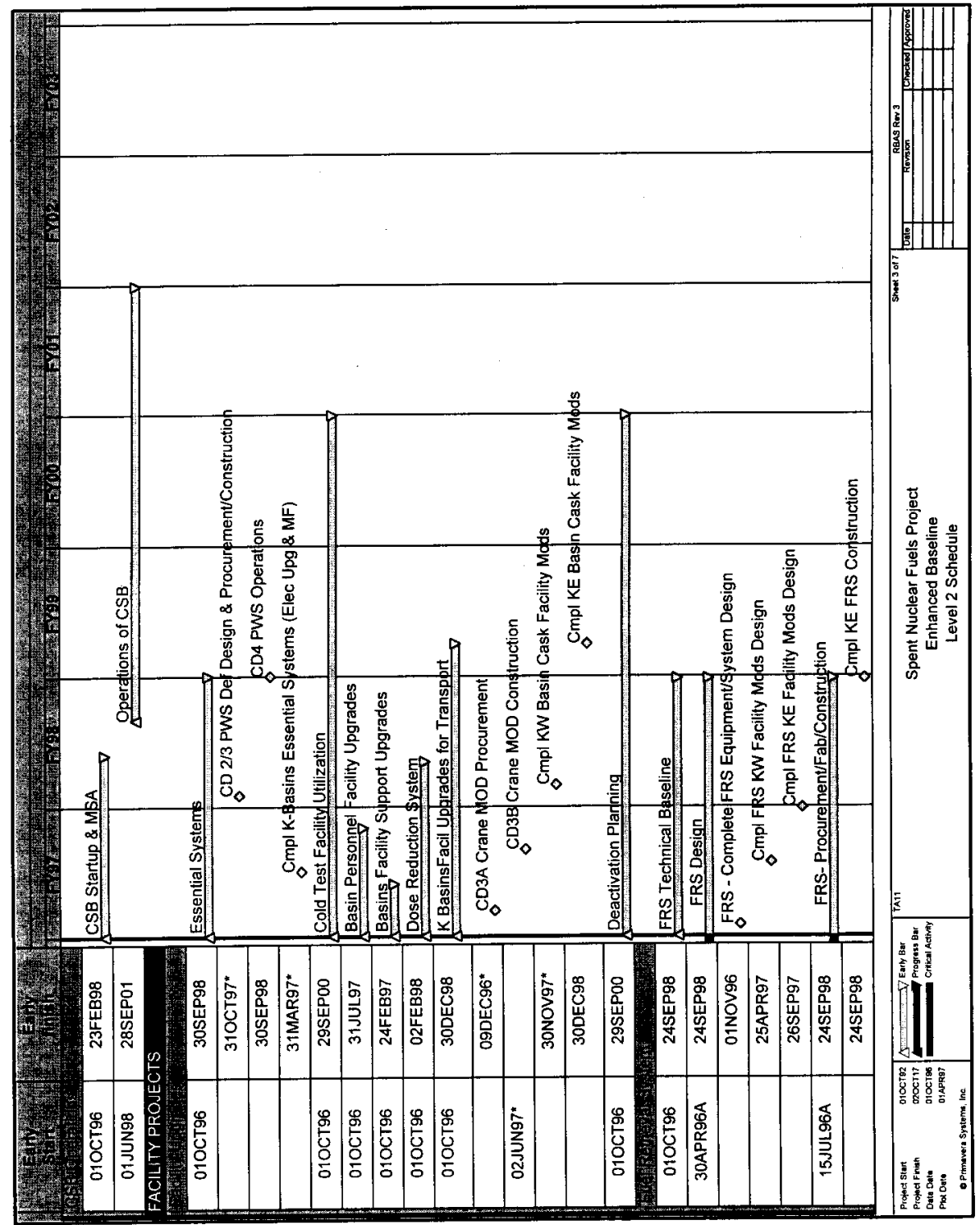




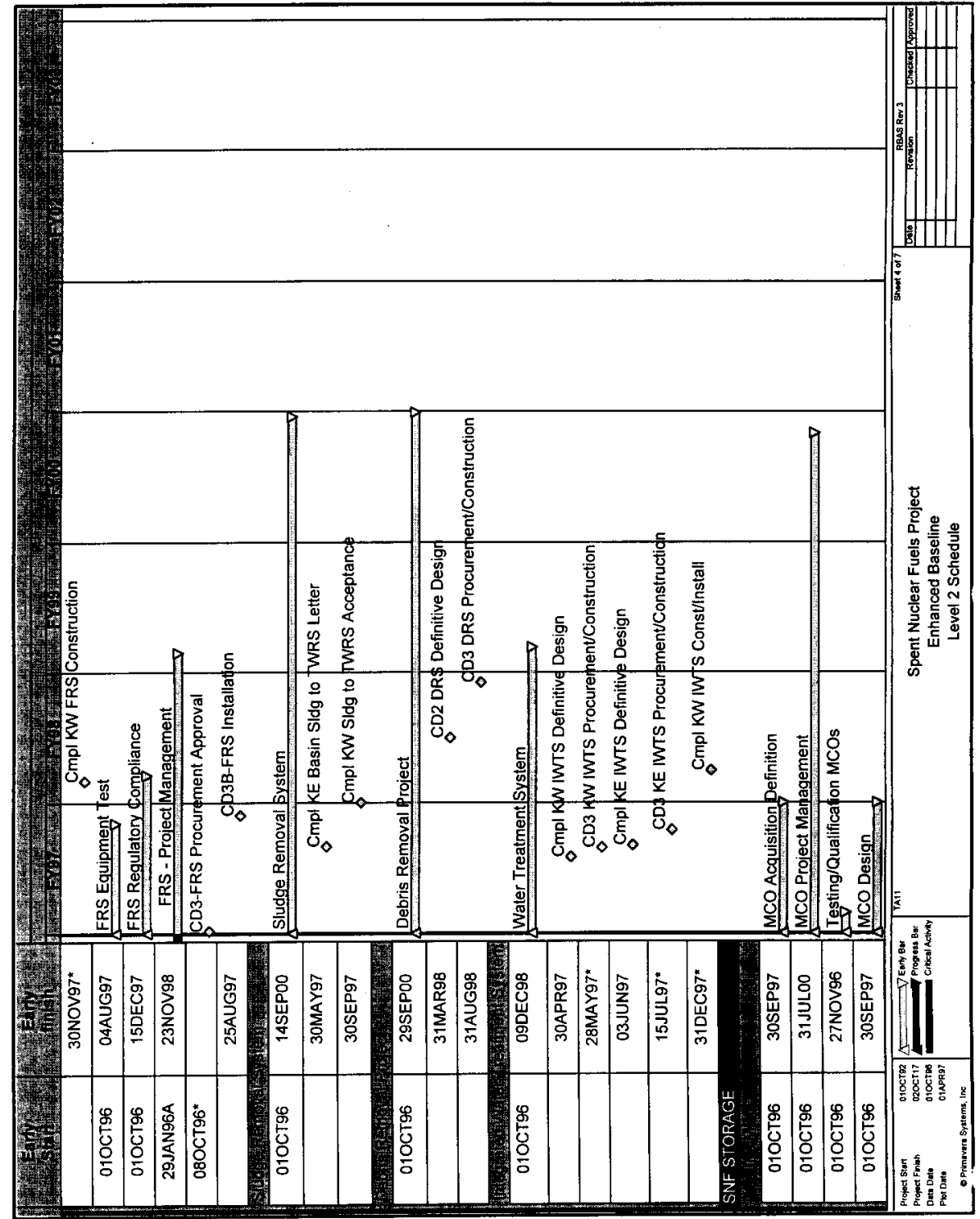


HNF-SP-1104, Rev 4

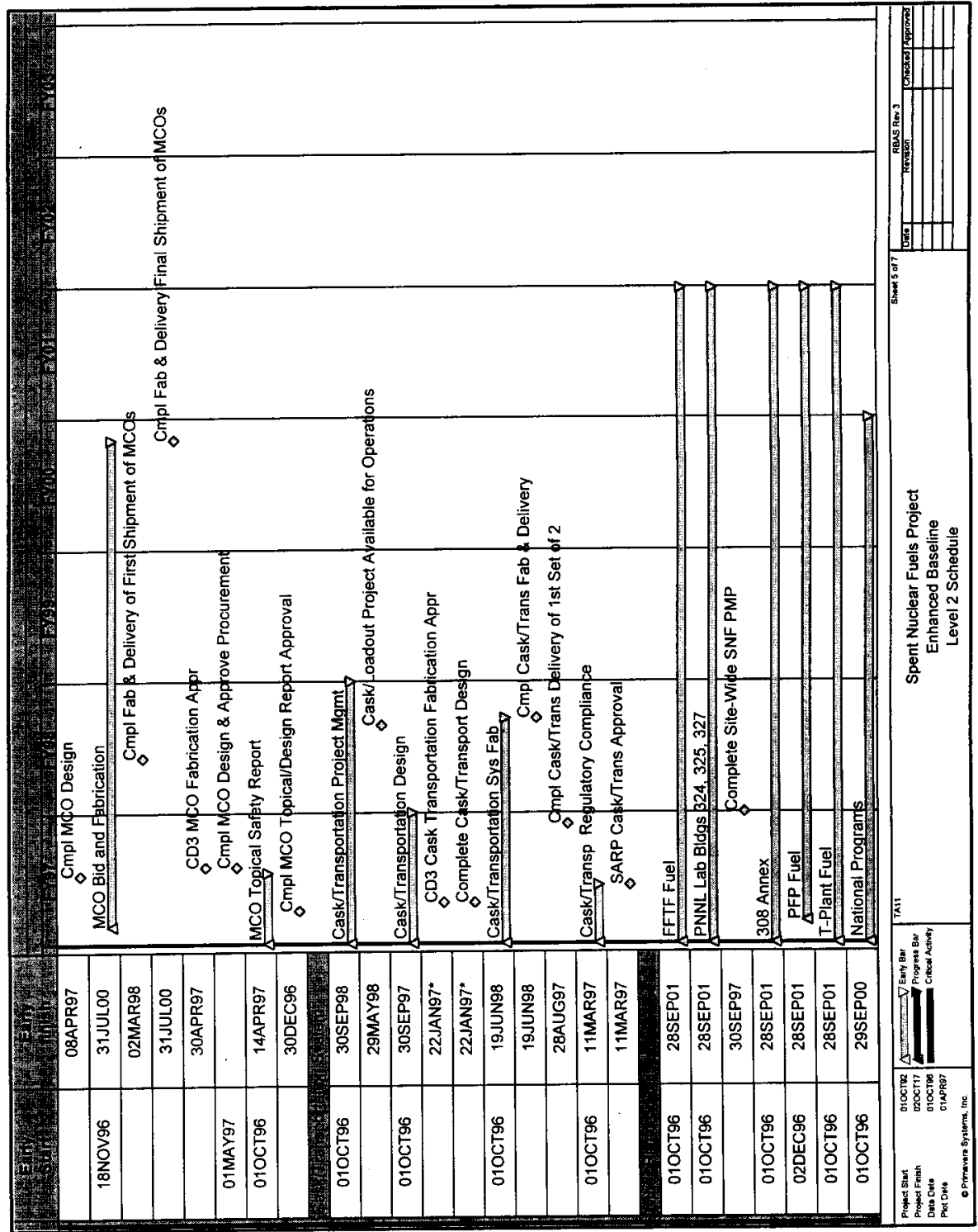




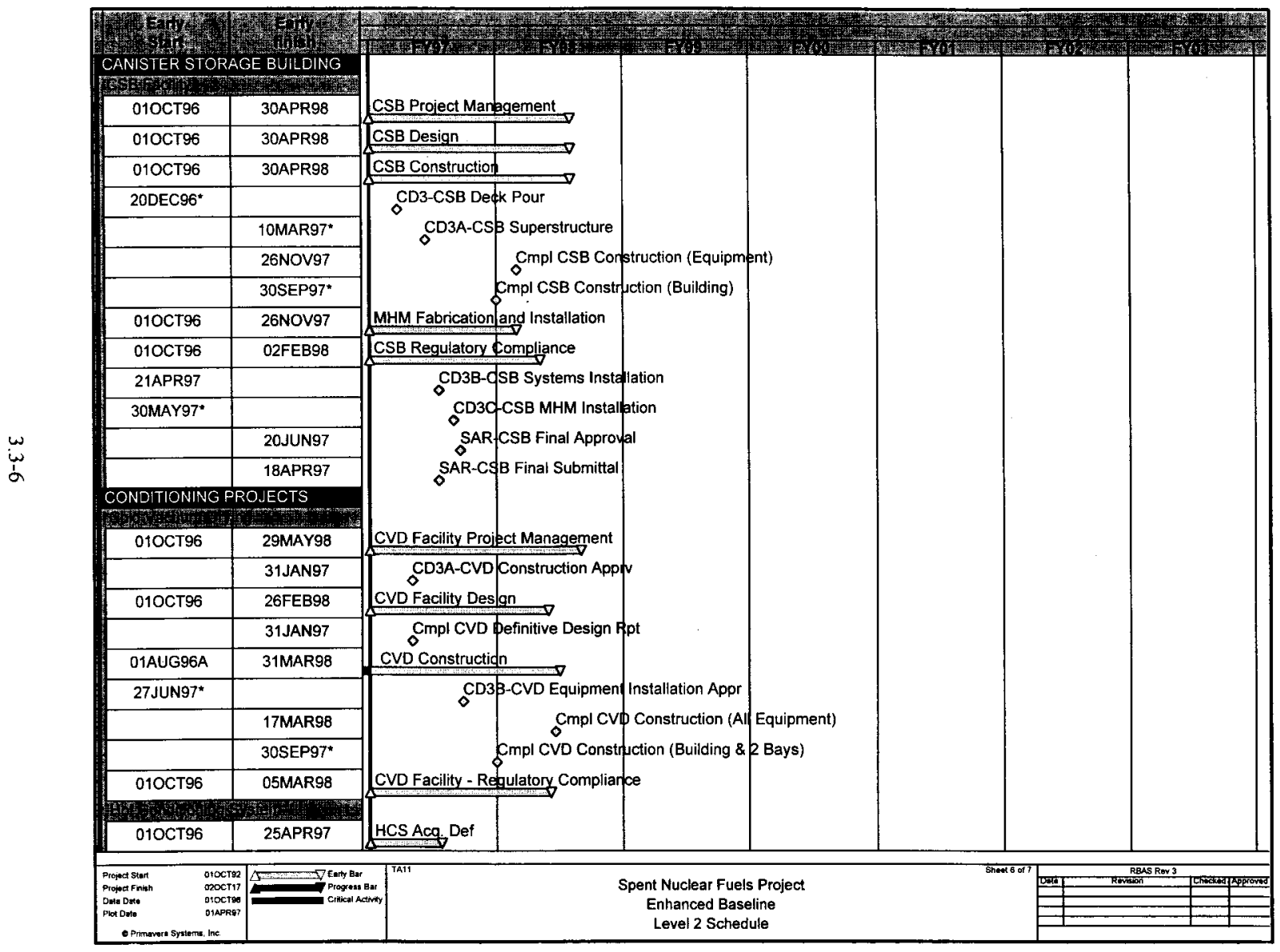




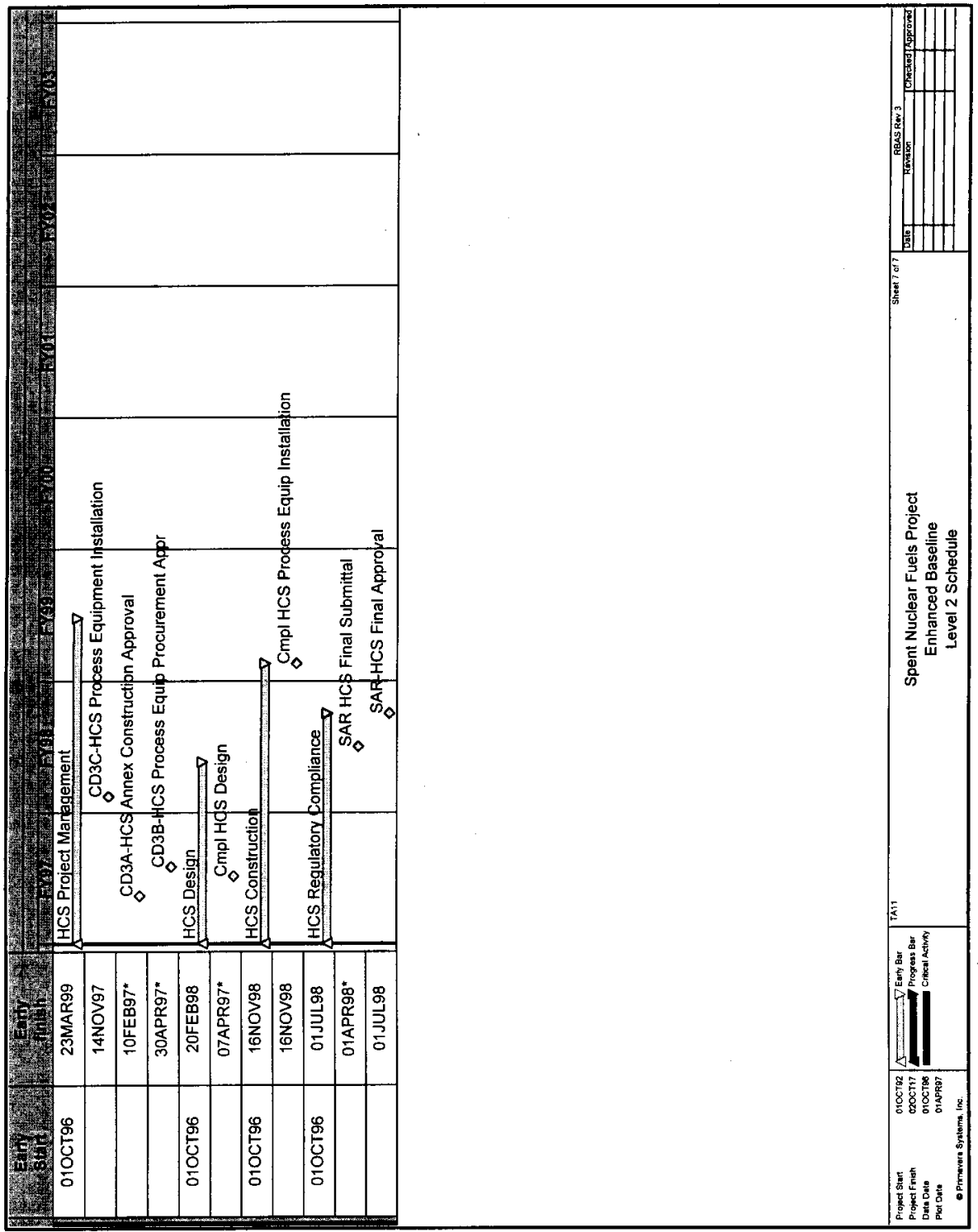




\section{SPENT NUCLEAR FUEL PROJECT}

WBS 1.4.1

HNF-SP-1 104, Rev 4

This page intentionally left blank. 


\begin{tabular}{|c|c|c|c|c|c|c|c|c|c|c|c|}
\hline & $\begin{array}{l}\text { MAP } \\
\text { MILSTOA } \\
\text { MS 1997 - PROD }\end{array}$ & $\mathrm{HE}$ & $\begin{array}{l}341 \\
\mathrm{HST} \\
\mathrm{SOH}\end{array}$ & 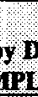 & $\begin{array}{l}\mathrm{gte} \\
\mathrm{ge}\end{array}$ & & & & & & \\
\hline Whis tone & With & & & inge & 21 & tot & & & Sertin: & Xinterot & \\
\hline Wha & & TA & DST & TO & $\mathrm{YM}$ & WAS & ST & Exh & 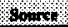 & mons & Data \\
\hline SO4-97-350 & CD3 FRS Procurement Approval & & & & & & & $\mathrm{x}$ & $\mathbf{M}$ & & $10 / 8 / 96$ \\
\hline SO4-97-515 & Complete FRS Equipment / System Design & $\mathrm{x}$ & & & & & $\mathrm{x}$ & $\mathrm{x}$ & $\mathbf{D}$ & & $11 / 1 / 96$ \\
\hline S06-97-020 & CD3A Crane MOD Procurement & & & & & & & $\mathbf{x}$ & N/A & & $12 / 9 / 96$ \\
\hline S07-97-046 & CD3 CSB Deck Pour & & & & & & & $\mathbf{x}$ & $\mathbf{M}$ & & $12 / 20 / 96$ \\
\hline S05-97-004 & Complete MCO Topical / Design Report Approval & & & & & & & $\mathrm{x}$ & D & & $12 / 30 / 96$ \\
\hline PA 5.1.1 & Complete Schedule Rebaseline & & & & & $\mathbf{x}$ & $\mathrm{x}$ & $\mathrm{x}$ & $P$ & & $12 / 31 / 96$ \\
\hline PA 5.1.2 & Complete Cost Savings Rebaseline & & & & & $\bar{x}$ & $\bar{x}$ & $\bar{x}$ & $P$ & & $12 / 31 / 96$ \\
\hline S06-97-011 & CD3 Cask Transportation Fabrication Approval & & & & & & & $\bar{x}$ & $\mathbf{M}$ & & $1 / 22 / 97$ \\
\hline PA 1.3.1 & Complete Cask/Trans Design & & & & $\mathbf{x}$ & $\mathrm{x}$ & $\bar{x}$ & $\bar{x}$ & $P$ & & $1 / 22 / 97$ \\
\hline S08-97-007 & CD3A CVD Construction Approval & & & & & & & $\bar{x}$ & $\mathbf{M}$ & & $1 / 31 / 97$ \\
\hline S08-97-006 & Complete CVD Definitive Design Report & & & & $x$ & & $\mathrm{x}$ & $\mathrm{X}$ & $\mathbf{D}$ & & $1 / 31 / 97$ \\
\hline S08-97-012 & CD3A HCS Annex Construction Approval & & & & & & & $\mathrm{x}$ & $\mathbf{M}$ & & $2 / 10 / 97$ \\
\hline S06-97-005 & SARP Cask/Trans Approval & & & & & & & $\bar{x}$ & $\mathbf{D}$ & & $3 / 11 / 97$ \\
\hline S04-97-104 & Complete K-Basins Essential Systems (elec upgrade \& MF) & & & & & & & $\mathbf{x}$ & D & & $3 / 31 / 97$ \\
\hline PA 2.2.1 & Complete HCS Design & & & & & $\mathrm{X}$ & $\mathrm{x}$ & $\mathbf{X}$ & $\mathrm{P}$ & & $4 / 7 / 97$ \\
\hline $505-97-007$ & Complete MCO Design & & & & $\mathbf{x}$ & & $\mathrm{x}$ & $\bar{x}$ & $\mathbf{D}$ & & $4 / 8 / 97$ \\
\hline S07-97-054 & SAR CSB Final Submittal & & $\bar{x}$ & & & & $\mathbf{x}$ & $\bar{x}$ & D & D-04 & $4 / 18 / 97$ \\
\hline S07-97-047 & CD3B CSB Systems Installation & & & & & & & $\overline{\mathrm{x}}$ & $\mathbf{M}$ & & $4 / 21 / 97$ \\
\hline S04-97-347 & Complete FRS KW Facility Mods Design & & & & $\mathrm{x}$ & & $\mathrm{x}$ & $\mathrm{X}$ & $\mathbf{D}$ & D-01 & $4 / 25 / 97$ \\
\hline S04-97-613 & Complete KW IWTS Definitive Design & & & & & & & $\overline{\mathrm{x}}$ & $\vec{D}$ & D-02 & $4 / 30 / 97$ \\
\hline S05-97-011 & CD3 MCO Fabrication Approval & & & & & & & $\bar{x}$ & $\mathbf{M}$ & & $4 / 30 / 97$ \\
\hline S08-97-008 & CD3B HCS Process Equip Procurement Approval & & & & & & & $\mathrm{X}$ & $\mathbf{M}$ & & $4 / 30 / 97$ \\
\hline PA 1.3 .2 & Complete MCO Design \& Approve Procurement & & & & & $\mathbf{x}$ & $\mathbf{x}$ & $\mathrm{x}$ & $\overline{\mathbf{P}}$ & & $5 / 1 / 97$ \\
\hline S03-97-052 & SAR K-Basin Rev 4/TSR, Rev I Submittal & & $\mathrm{X}$ & & & & $\overline{\mathbf{x}}$ & $\mathrm{x}$ & $\mathrm{D}$ & & $5 / 27 / 97$ \\
\hline S04-97-609 & CD3 KW IWTS Procurement / Construction & & & & & & & $\bar{x}$ & $\vec{M}$ & & $5 / 28 / 97$ \\
\hline PA 3.1.1 & Complete KE Basin Sludge to TWRS letter & & & & & $\mathbf{x}$ & $\mathrm{x}$ & $\bar{x}$ & $\mathbf{P}$ & & $5 / 30 / 97$ \\
\hline S07-97-048 & CD3C CSB MHM Installation & & & & & & & $\mathrm{X}$ & $\bar{M}$ & & $5 / 30 / 97$ \\
\hline S06-97-021 & CD3B Crane MOD Construction & & & & & & & $\mathrm{x}$ & $\bar{M}$ & & $6 / 2 / 97$ \\
\hline S04-97-615 & Complete KE IWTS Definitive Design & & & & & & & $\bar{x}$ & $\mathrm{D}$ & D-02 & $6 / 3 / 97$ \\
\hline S07-97-053 & SAR CSB Final Approval & & $\bar{x}$ & & & & $\bar{x}$ & $\mathrm{x}$ & $\mathrm{s}$ & & $6 / 20 / 97$ \\
\hline $508-97-020$ & CD3B CVD Equipment Installation Approval & & & & & & & $\bar{x}$ & $\bar{M}$ & & $6 / 27 / 97$ \\
\hline
\end{tabular}

\section{"Criteria Definition:}

$\mathrm{S}=$ Stakeholder Document (TPA, DNFSB, VA)

$\mathrm{P}=$ Performance Agreement

$M=$ Milestone Description Sheet

$\mathrm{D}=$ Dictionary Definition

(In definition process, examples attached) 


\begin{tabular}{|c|c|c|c|c|c|c|c|c|c|c|c|}
\hline$\sqrt{10}$ & 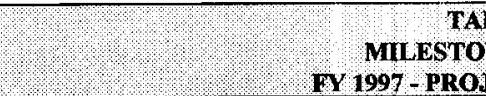 & $\mathrm{WE}$ & $\begin{array}{l}341 \\
\mathrm{SOb}\end{array}$ & III & 19 & [OI & & & & & \\
\hline Trilowo & wito & & & 64 & 6 & 3 & & & Ginth & Dinfition? & \\
\hline 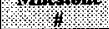 & 8 & Wx & DNSSB & Wo: & W. & (1) & St) & Now & onom & por $\mathrm{xyp}$ & $0 \times \mathbb{N E}$ \\
\hline S04-97-616 & CD3 KE IWTS Procurement / Construction & & & & & & & $\bar{x}$ & $\mathbf{M}$ & & $7 / 15 / 97$ \\
\hline S04-97-352 & CD3B FRS Installation & & & & & & & $\bar{x}$ & $\mathbf{M}$ & & $8 / 25 / 97$ \\
\hline S03-97-055 & SAR K-Basin Rev 4/TSR, Rev 1 Approval & $\mathrm{x}$ & & & & & $\mathbf{x}$ & $\mathrm{X}$ & $\mathbf{S}$ & & $8 / 27 / 97$ \\
\hline S06-97-050 & Complete Cask/Trans Delivery of 1st Set of 2 & & & & & & & $\bar{x}$ & $\mathrm{D}$ & & $8 / 28 / 97$ \\
\hline$S 04-97-348$ & Complete FRS KE Facility Mods Design & & & & $\bar{x}$ & & $\mathrm{x}$ & $\mathrm{x}$ & $\overline{\mathrm{D}}$ & D-01 & $9 / 26 / 97$ \\
\hline PA 3.1.1 & Complete KW Sldg to TWRS Acceptance & & & & & $\bar{x}$ & $\mathrm{x}$ & $\mathrm{x}$ & $P$ & & $9 / 30 / 97$ \\
\hline PA 3.1.2 & Complete KE Empty Canister \& South Load Out Pit & & & & & $\mathrm{x}$ & $\mathrm{x}$ & $\mathrm{x}$ & $P$ & & $9 / 30 / 97$ \\
\hline PA 1.2.1 & Complete CSB Construction (Building) & & & & & $\mathrm{x}$ & $\mathrm{x}$ & $\bar{x}$ & $P$ & & $9 / 30 / 97$ \\
\hline PA 1.4.1 & Complete CVD Construction (Building \& 2 bays) & & & & & $\bar{x}$ & $\bar{x}$ & $\mathrm{x}$ & $P$ & & $9 / 30 / 97$ \\
\hline PA 4.1.1 & Complete Site-Wide SNF PMP & & & & & $\mathrm{X}$ & $\mathrm{x}$ & $\mathrm{X}$ & $P$ & & $9 / 30 / 97$ \\
\hline S04-98-105 & $\begin{array}{l}\text { CD2/3 PWS Definitive Design \& Procurement / } \\
\text { Construction }\end{array}$ & & & & & & & $\bar{x}$ & $\mathbf{M}$ & & $10 / 31 / 97$ \\
\hline S08-98-025 & CD3C HCS Process Equipment Installation & & & & & & & $\bar{x}$ & $\mathbf{M}$ & & $11 / 14 / 97$ \\
\hline S07.98-050 & Complete CSB Construction (Equipment) & & & & & & & $\bar{X}$ & D & & $11 / 26 / 97$ \\
\hline PA 1.1.1 & Complete KW FRS Constnuction & $\mathrm{x}$ & & & & $x$ & $\mathrm{x}$ & $\bar{x}$ & $P$ & & $11 / 30 / 97$ \\
\hline PA 1.1.2 & Complete KW Basin Cask Facility Mods & & & & & $x$ & $\mathrm{x}$ & $\mathrm{X}$ & $\bar{P}$ & & $11 / 30 / 97$ \\
\hline PA 1.1.4 & Complete KW IWTS Construction /Installation & $x$ & & & & $\mathrm{x}$ & $\mathrm{x}$ & $\mathrm{x}$ & $P$ & & $12 / 31 / 97$ \\
\hline S04-98-607 & CD4 KW IWTS Operations & & & & & & & $\bar{x}$ & $\mathbf{M}$ & & $2 / 26 / 98$ \\
\hline S05-98-010 & Complete Fab \& Delivery First Shipment of MCO's & & & & & & & $\bar{x}$ & $\mathbf{D}$ & D-03 & $3 / 2 / 98$ \\
\hline S08-97-220 & Complete CVD Construction (all equipment) & & & & & & & $\mathrm{X}$ & D & & $3 / 17 / 98$ \\
\hline S04-98-510 & CD2 DRS Definitive Design & & & & & & & $x$ & $\mathbf{M}$ & & $3 / 31 / 98$ \\
\hline S08-98-020 & SAR HCS Final Submittal & & & & & & & $\mathrm{X}$ & $\mathrm{D}$ & D-04 & $4 / 1 / 98$ \\
\hline S03-98-602 & Complete SNFP Contractor Operations Readiness Review & & $\bar{x}$ & & & & $x$ & $\mathrm{x}$ & $\mathrm{S}$ & & $4 / 24 / 98$ \\
\hline$\$ 03-98-625$ & Complete SNFP DOE Operations Readiness Review & & $x$ & & & & $\mathrm{x}$ & $\bar{x}$ & s & & $5 / 29 / 98$ \\
\hline $\mathbf{S 0 3 - 9 8 - 6 2 0}$ & CD4 Fuel Movement Operations (KW) CSB, CVD, FRS & & $\mathrm{x}$ & & $\mathrm{x}$ & & $\mathrm{x}$ & $\bar{x}$ & $\mathbf{M}$ & & $\$ / 30 / 98$ \\
\hline PA 1.1.3 & Start Fuel Removal Operations & $x$ & $\mathrm{x}$ & $x$ & & $\mathrm{x}$ & $\mathrm{x}$ & $\bar{x}$ & $P$ & & $5 / 31 / 98$ \\
\hline S06-98-006 & Complete Cask/Trans Fabrication \& Delivery & & & & & & & $\bar{x}$ & $\mathrm{D}$ & & $6 / 19 / 98$ \\
\hline S08-98-018 & SAR HCS Final Approval & & & & & & & $\bar{x}$ & $\mathbf{D}$ & D-04 & $7 / 1 / 98$ \\
\hline$S 04-98-511$ & CD3 DRS Procurement / Construction & & & & & & & $\bar{x}$ & $\mathrm{M}$ & & $8 / 31 / 98$ \\
\hline$\$ 04-97-101$ & CD4 PWS Operations & & & & & & & $\mathrm{x}$ & $M$ & & $9 / 30 / 98$ \\
\hline S04-98-356 & Complete KE FRS Construction & $\bar{x}$ & & & & & $\mathrm{x}$ & $x$ & s & & $9 / 24 / 98$ \\
\hline
\end{tabular}

*Criteria Definition:

$S=$ Stakeholder Document (TPA, DNFSB, VA)

$\mathrm{P}=$ Performance Agreement

$M=$ Milestone Description Sheet

$\mathrm{D}=$ Dictionary Definition

(in definition process, examples attached) 


\begin{tabular}{|c|c|c|c|c|c|c|c|c|c|c|c|}
\hline \multirow{3}{*}{ Wै। } & \multicolumn{8}{|c|}{ 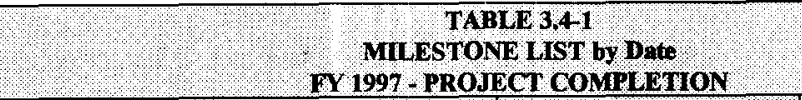 } & & 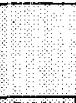 & \multirow[b]{3}{*}{$\mathrm{bat}$} \\
\hline & \multirow{2}{*}{ Nites } & \multicolumn{6}{|c|}{$\mathrm{NaHO} / \mathrm{H}$} & & \multicolumn{2}{|c|}{ GHten in phition } & \\
\hline & & 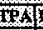 & WTS & $\mathbf{1 0}$ & $\times$ & es & 1968 & inst & $80+1$ & $10+1$ yom & \\
\hline S08-98-009 & Complete HCS Process Equipment Installation & & & & & & & $\mathrm{x}$ & $\mathrm{D}$ & & $11 / 16 / 98$ \\
\hline S04-97-621 & Complete KE IWTS ATP & & & & & $\mathrm{x}$ & $\overline{\mathrm{x}}$ & $\bar{x}$ & s & & $12 / 9 / 98$ \\
\hline S06-97-012 & Complete KE Basin Cask Facility Mods & & & & & & & $\overline{\mathrm{X}}$ & $\overline{\mathrm{D}}$ & & $12 / 30 / 98$ \\
\hline$\$ 04-99-510$ & CDAA Fuel Movement Operations (KE) & & & & & & & $\bar{x}$ & $\bar{M}$ & & $1 / 28 / 99$ \\
\hline S04-98-608 & CD4 KE IWTS Operations & & & & & & & $\bar{X}$ & $\mathbf{M}$ & & $1 / 29 / 99$ \\
\hline S08-98-013 & CD4 HCS Operations & & & & & & & $\overline{\mathrm{X}}$ & $\mathbf{M}$ & & $3 / 23 / 99$ \\
\hline$\$ 04-00-520$ & CD4 DRS Operations & & & & & & & $\bar{x}$ & $\overrightarrow{\mathbf{M}}$ & & $10 / 4 / 99$ \\
\hline S00-00-902 & Complete Fuel Removal from Basins & $\mathrm{x}$ & $\bar{x}$ & $\bar{x}$ & $\bar{x}$ & & $\mathrm{x}$ & $\mathrm{x}$ & $\mathrm{S}$ & & $7 / 31 / 00$ \\
\hline S05-99-001 & Complete Fab \& Delivery Final Shipment of MCO's & & & & & & & $\mathrm{x}$ & $\mathrm{D}$ & $\overline{\mathrm{D}-03}$ & $7 / 31 / 00$ \\
\hline S04-00-205 & Start Sludge Transfer from KE-Basin to TWRS & $\mathrm{x}$ & $\bar{x}$ & & & & $x$ & $\mathrm{x}$ & s & & $9 / 29 / 00$ \\
\hline$\$ 04-01-507$ & Complete Basin Debris Removal & $\bar{x}$ & & & & & $\bar{x}$ & $x$ & s & & $2 / 26 / 01$ \\
\hline S08-02-001 & Complete HCS Operations (Fuel in Dry Storage) & & & & & & $\mathrm{x}$ & $\bar{x}$ & D & & $3 / 22 / 01$ \\
\hline S04-01-215 & Complete Sludge Removal & $\bar{x}$ & $\bar{x}$ & $\bar{x}$ & $\bar{x}$ & & $\bar{x}$ & $\mathrm{X}$ & s & & $8 / 30 / 01$ \\
\hline$\$ 00-01-909$ & Complete Spent Nuclear Fuel Project & & & $\bar{x}$ & & & $\bar{x}$ & $\mathrm{X}$ & $\mathrm{D}$ & & $9 / 30 / 01$ \\
\hline S07-97-044 & CD3A CSB Superstructure & & & & & & & $\mathrm{x}$ & $\mathrm{M}$ & & $3 / 10 / 97$ \\
\hline
\end{tabular}

-Criteria Definition: 


\section{DRAFT ONLY - EXAMPLE PURPOSES Dictionary Definition}

\begin{tabular}{|c|c|c|c|}
\hline $\begin{array}{l}\text { Def } \\
\text { Type }\end{array}$ & Titie & $\begin{array}{c}\text { Description of what constitutes completion of } \\
\text { this milestone }\end{array}$ & $\begin{array}{l}\text { Applicable } \\
\text { Milestone \# }\end{array}$ \\
\hline D-01 & Facility Design & $\begin{array}{l}\text { This milestone is complete when FDNW transmits final design } \\
\text { drawings and specifications necessary to procure, fabricate and } \\
\text { install the equipment and hardware to DESH. ATP's to be } \\
\text { prepared under/during Title III Engineering during construction. } \\
\text { (Drawings) }\end{array}$ & $\begin{array}{l}\text { S04-97-347 } \\
\text { S04-97-348 }\end{array}$ \\
\hline D-02 & Definitive Design & $\begin{array}{l}\text { Completion of approved design media as described above and } \\
\text { transmitted to } \mathrm{K} \text { Basin Projects. (Drawings and Other) }\end{array}$ & $\begin{array}{l}\text { S04-97-613 } \\
\text { S04-97-613 }\end{array}$ \\
\hline D-03 & $\begin{array}{l}\text { Fab \& Delivery Shipment } \\
\text { MCO's }\end{array}$ & Receipt of shipment at warehousing. (Other) & $\begin{array}{l}\text { S05-98-010 } \\
\text { S05-99-001 }\end{array}$ \\
\hline D-04 & SAR Final Submittal & $\begin{array}{l}\text { This milestone is complete when DESH approves and submits the } \\
\text { SAR to DOE-RL. (Letter) }\end{array}$ & $\begin{array}{l}\text { S07-97-026 } \\
\text { S07-98-018 }\end{array}$ \\
\hline & & & \\
\hline & & & \\
\hline & & & \\
\hline & & & \\
\hline
\end{tabular}




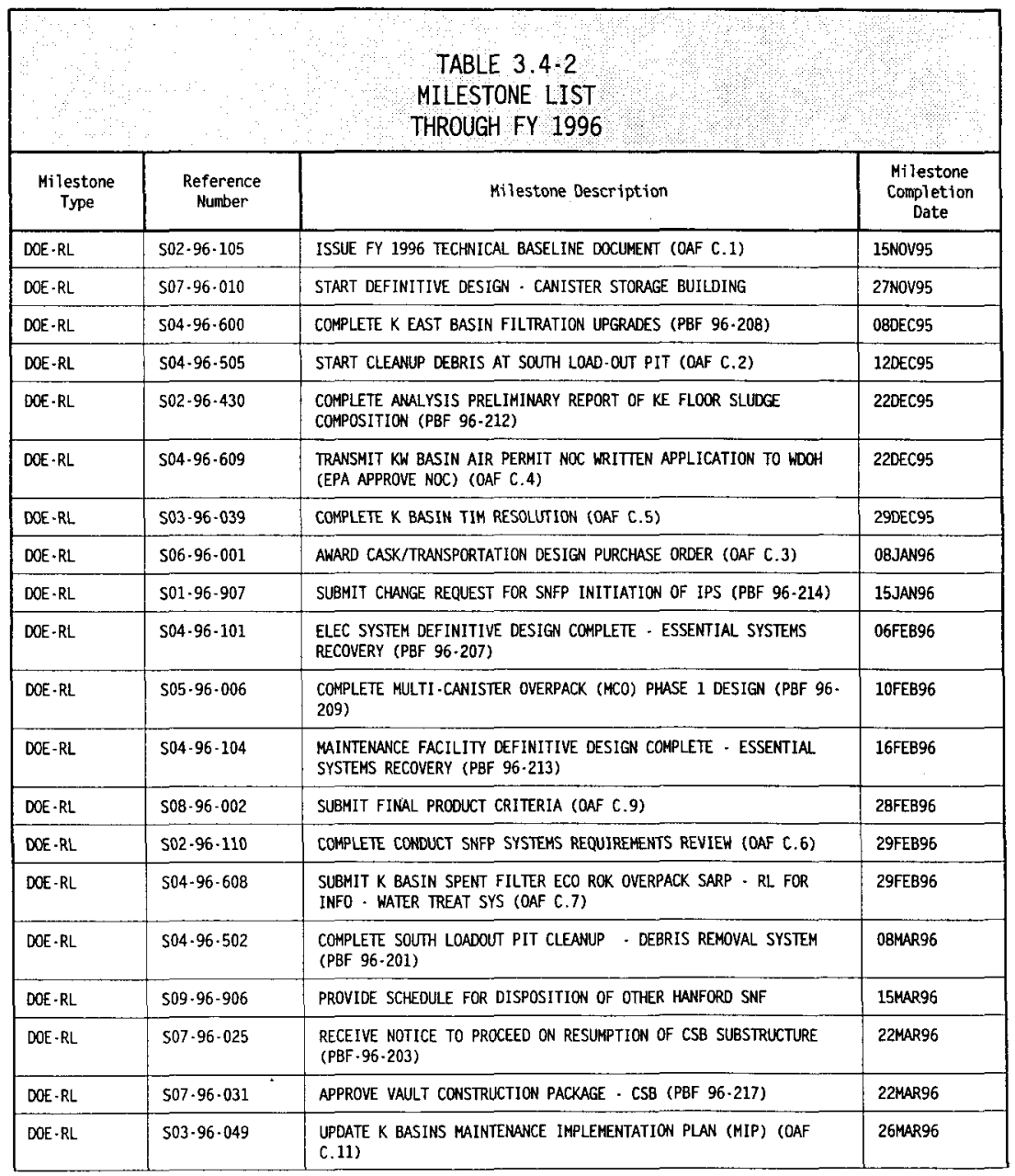


TABLE $3.4 \% 2$

MILESTONE LIST

THROUGH FY 1996

\begin{tabular}{|c|c|c|c|}
\hline $\begin{array}{l}\text { Milèstone } \\
\text { Type }\end{array}$ & $\begin{array}{l}\text { Reference } \\
\text { Number }\end{array}$ & Milestone Description & $\begin{array}{l}\text { Milestone } \\
\text { Completion } \\
\text { Date }\end{array}$ \\
\hline DOE $\cdot R L$ & $508-96-003$ & $\begin{array}{l}\text { PROVIDE INPUT TO TECHNICAL REQUIREMENTS FOR DESIGN - } \\
\text { CONDITIONING (OAF C.10) }\end{array}$ & 28MAR96 \\
\hline DOE-RL & $503-96.048$ & $\begin{array}{l}\text { COMPLETE K BASINS ESSENTIAL DRAWINGS AND SYS DESCRIPTION (PBF } \\
96-206 \text { ) }\end{array}$ & 29MAR96 \\
\hline DOE-RL & 508.96 .008 & $\begin{array}{l}\text { COMPLETE CONCEPTUAL DESIGN REPORT FOR COLD VACUUM DRYING } \\
\text { SYSTEM (PBF 96-215) }\end{array}$ & 29MAR96 \\
\hline $\mathrm{DOE} \cdot \mathrm{RL}$ & $504-96 \cdot 107$ & $\begin{array}{l}\text { OBTAIN SLDG MOU SIGN BY TWRS, SNFP (WHC), \& TWRS \& SFD (RL) } \\
\text { SLUDG REMOVE SYS (OAF C.12) }\end{array}$ & 29MAR96 \\
\hline DOE-RL & S02-96-225 & ISSUE SNF PROCESS FLOW DIAGRAM & 31MAR96 \\
\hline $\mathrm{DOE} \cdot \mathrm{RL}$ & $504-96 \cdot 340$ & START FRS FINAL DESIGN - CD $1 / 2$ & 01APR96 \\
\hline DOE-RL & S02.96-466 & 327 FACILITY APPROVED FOR RECEIPT KE FUEL AND CANISTER SLUDGE & O1APR96 \\
\hline DOE-RL & $508 \cdot 96 \cdot 004$ & $\begin{array}{l}\text { SUBMIT REV. O PERFORMANCE SPECIFICATIONS FOR COLD VACUUM } \\
\text { DRYING SYSTEM }\end{array}$ & 01APR96 \\
\hline$D O E-R L$ & $508 \cdot 96 \cdot 005$ & START DEFINITIVE DESIGN FOR COLD VACUUM DRYING SYSTEM & OLAPR96 \\
\hline DOE-RL & $508 \cdot 96 \cdot 010$ & $\begin{array}{l}\text { CRITICAL DECISION (CD-1/2) TO PROCEED WITH DEFINITIVE DESIGN } \\
\text { OF THE CVD FACILITY }\end{array}$ & 01APR96 \\
\hline DOE-RL & $506-96-005$ & COMPLETE CASK PRELIMINARY DESIGN (PBF 96.202) & 25APR96 \\
\hline DOE-RL & $\$ 01 \cdot 96 \cdot 111$ & ISSUE THE FY 1998 ACTIVITIES DATA SHEETS & 29APR96 \\
\hline $\mathrm{DOE} \cdot \mathrm{RL}$ & $504 \cdot 96 \cdot 325$ & FRS - PROJECT MANAGEMENT PLAN & 01MAY96 \\
\hline DOE-RL & $504 \cdot 96 \cdot 333$ & SUBMIT FRS CONCEPTUAL DESIGN REPORT TO DOE (PBF 96-211) & 07MAY96 \\
\hline DOE-RL & $506 \cdot 96-007$ & $\begin{array}{l}\text { DEVELOP CASK/TRANSPORTATION DRAFT SAFETY ANALYSIS REPORT FOR } \\
\text { PACKAGING (SARP) }\end{array}$ & 16 MAY96 \\
\hline DOE-RL & $507 \cdot 96 \cdot 015$ & START OF PROCUREMENT · CSB & 29 MAY96 \\
\hline $\mathrm{DOE} \cdot \mathrm{RL}$ & $502-96 \cdot 415$ & $\begin{array}{l}\text { COMPLETE SHIPMENT OF KE FUEL AND CANISTER SLUDGE TO HOT CELL } \\
\text { (PBF } 96-210)\end{array}$ & 31 MAY96 \\
\hline DOE $\cdot R L$ & $\$ 05-96.007$ & COMPLETE MCO TESTING (OAF) & 31MAY96 \\
\hline DOE $\cdot$ RL & $504 \cdot 96 \cdot 204$ & $\begin{array}{l}\text { CLEAN AND PAINT BASIN WALLS COMPLETE - DOSE REDUCTION (PBF } 96 \text { - } \\
205 \text { ) }\end{array}$ & 17 JUN96 \\
\hline DOE $\cdot R L$ & $502 \cdot 96 \cdot 425$ & ISSUE DRYING DATA REPORT FOR PROCESS DEFINITION & 28JUN96 \\
\hline DOE-RL & $\$ 04 \cdot 96-110$ & ESTABLISH ACCEPTABILITY OF 105-KE FLOOR SLUDGE BY TWRS & 283UN96 \\
\hline DOE-RL & $507.96 \cdot 037$ & $\begin{array}{l}\text { PHASE II SAR FOR AUTHORIZATION OF CSB OPER DECK, TUBES, REC } \\
\text { CRANE, \& MCO HANDLING MACHINE }\end{array}$ & 28JUN96 \\
\hline DOE-RL & $507 \cdot 96-011$ & $\begin{array}{l}\text { COMPLETE DEFINITIVE DESIGN - CANISTER STORAGE BUILOING (PBF } \\
96-204)\end{array}$ & 26JUน 96 \\
\hline$D O E-R L$ & S08-96-009 & $\begin{array}{l}\text { CMPLT CONCEPT DESIGN FOR HOT CONDITIONING SYSTEM PROCESS } \\
\text { EQUIPMENT (PBF } 96 \cdot 216 \text { ) }\end{array}$ & OJAUG96 \\
\hline
\end{tabular}




\section{SPENT NUCLEAR FUEL PROJECT \\ WBS 1.4 .1}

TABLE $3.4-2$

MILESTONE LIST

THROUGH FY 1996

\begin{tabular}{|c|c|c|c|}
\hline $\begin{array}{l}\text { Milestone } \\
\text { Type }\end{array}$ & $\begin{array}{l}\text { Reference } \\
\text { Number }\end{array}$ & Milestone Description & $\begin{array}{l}\text { Milestone } \\
\text { Completion } \\
\text { Date }\end{array}$ \\
\hline DOE-RL & S08-96.011 & $\begin{array}{l}\text { CRITICAL DECISION (CD-1/2) TO PROCEED WITH DEFIN DSGN OF THE } \\
\text { CBS-HCS PROCESS EQUIP'T }\end{array}$ & $02 A U G 96$ \\
\hline DOE -RL & $507 \cdot 96 \cdot 038$ & $\begin{array}{l}\text { PHASE III SAR FOR AUTH OF CSB SUPERSTRUCTURE ERECT AND FAB OF } \\
\text { SAFETY CLASS I OR II AUX EQUIP }\end{array}$ & 15AUG96 \\
\hline $\mathrm{DOE} \cdot \mathrm{RL}$ & $508-96 \cdot 030$ & $\begin{array}{l}\text { PHASE I SAR FOR AUTHORIZATION OF CIVIL/STRUCTURAL PORTION OF } \\
\text { HOT CONDITIONING ANNEX CONST }\end{array}$ & 15AUG96 \\
\hline $\mathrm{DOE} \cdot \mathrm{RL}$ & $\$ 08-96.007$ & START DEFINITIVE DESIGN - HOT CONDITIONING SYSTEM & 23AUG96 \\
\hline DOE-RL & 508.96 .006 & $\begin{array}{l}\text { SUBMIT REV O PERFORMANCE SPECIFICATIONS FOR HOT CONDITIONING } \\
\text { SYSTEM }\end{array}$ & 26AUG96 \\
\hline DOE $\cdot R L$ & $\$ 02-96 \cdot 210$ & INTEGRATEO REPORT FOR MCO PRESSURIZATION ANALYSIS (OAF) & 30ALIG96 \\
\hline $\mathrm{DOE} \cdot \mathrm{RL}$ & $504-96 \cdot 112$ & $\begin{array}{l}\text { DETERHINE TECHNICAL ACCEPTABILITY OF KE FLOOR SLUDGE FOR } \\
\text { TRANSFER TO TANK FARHS (OAF) }\end{array}$ & 30AUG96 \\
\hline DOE $\cdot R L$ & $508 \cdot 96 \cdot 013$ & COMPLETE CVD CILIL/STRUCT SAR DOCUMENTATION (OAF) & 31AUG96 \\
\hline DOE-RL & $501 \cdot 96 \cdot 211$ & ISSUE THE FIMAL PROGRAM PLAN (FY 97) & 03SEP96 \\
\hline $\mathrm{DOE} \cdot \mathrm{RL}$ & 500.96 .908 & DOCUMENT THE SNF PROJECT TECHNICAL BASIS (OAF) & 15SEP96 \\
\hline DOE $\cdot R L$ & $506-96-010$ & COMPLETE CASK/TRANSPORTATION PERFORMANCE TESTING & 19SEP96 \\
\hline $\mathrm{DOE} \cdot \mathrm{RL}$ & S06.96.013 & $\begin{array}{l}\text { CRITICAL DECISION (CD-1/2) TO PROCEED WITH CASK DEFINITIVE } \\
\text { DESIGN }\end{array}$ & 26SEP96 \\
\hline DOE $\cdot R L$ & $505-96-011$ & $\begin{array}{l}\text { CRITICAL DECISION }(C D \cdot 1 / 2) \text { TO PROCEED WITH MCO DEFINITIVE } \\
\text { DESIGN }\end{array}$ & 30SEP96 \\
\hline
\end{tabular}


This page intentionally left blank. 


\section{SPENT NUCLEAR FUEL PROJECT \\ WBS 1.4.1}

HNF-SP-1104, Rev 4

\begin{tabular}{|c|c|c|c|}
\hline \multicolumn{4}{|c|}{$\begin{array}{l}\text { Westinghouse Hanford Company } \\
\text { MILESTONE DESCRIPTION SHEET }\end{array}$} \\
\hline \multicolumn{3}{|c|}{ Title: Initiate FRS Procurement $\mathrm{CD}-3$} & Date: $01 / 26 / 96$ \\
\hline \multicolumn{3}{|c|}{ Assigned To: Spent Nuclear Fuel Project } & CIN: SNF $-96-095$ \\
\hline \multicolumn{3}{|c|}{ Program WBS Designator: 1.4.1.04.03.02.01.01 } & Due Date: $10 / 08 / 96$ \\
\hline \multicolumn{3}{|c|}{ Control Number: S04-97-350 } & Rev: 1 \\
\hline $\begin{array}{l}\text { MILESTONE TYPE: } \\
\text { DOE-HQ } \\
\text { X DOE-RL } \\
\text { D CONTRACTOR }\end{array}$ & $\begin{array}{l}\quad \text { DIVISION: } \\
\text { State } \\
\text { C Federal } \\
X \text { DOE } \\
\text { RCRA } \\
\square \text { TPA Number }\end{array}$ & $\begin{array}{l}\quad \text { DELIVERABLE: } \\
\text { Report } \\
\square \text { Letter } \\
\text { Drawings } \\
\text { X Other (specify) } \\
\text { Memorandum }\end{array}$ & $\begin{array}{l}\text { ADDRESS TO: } \\
\text { D DOE-HQ } \\
X \text { DOE-RL } \\
\square \text { Other (specify) }\end{array}$ \\
\hline \multicolumn{4}{|c|}{$\begin{array}{l}\text { Milestone Description: Initiation of Procurement for the equipment, hardware } \\
\text { and components necessary to complete fabrication and installation of process } \\
\text { systems in KW by August 29. } 1997 \text {. }\end{array}$} \\
\hline \multicolumn{4}{|c|}{$\begin{array}{l}\text { Description of what constitutes completion of this milestone: This milestone is } \\
\text { complete when wHC delivers the documentation necessary for a Critical Decision } \\
\text { presentation to the acquisition executive in accordance with the draft guidance } \\
\text { for performing CD's. } \\
\text { critical Decision is required by October 8, } 1996 \text { to maintain schedule } \\
\text { objectives of the Fuel Retrieval Sub-Project. }\end{array}$} \\
\hline \multicolumn{4}{|c|}{ Milestone Description Approval } \\
\hline \multicolumn{3}{|c|}{ 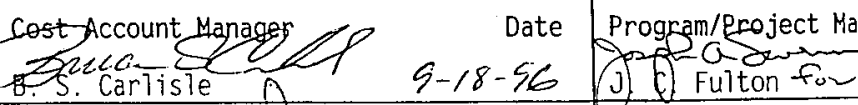 } & Dager $9 / 19 / 96$ \\
\hline \multicolumn{4}{|c|}{ Program Element Mangagr . D. Wiemers } \\
\hline \multicolumn{4}{|c|}{ 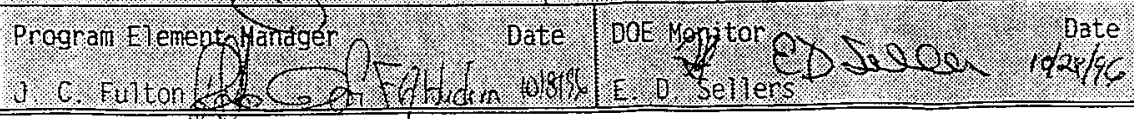 } \\
\hline
\end{tabular}




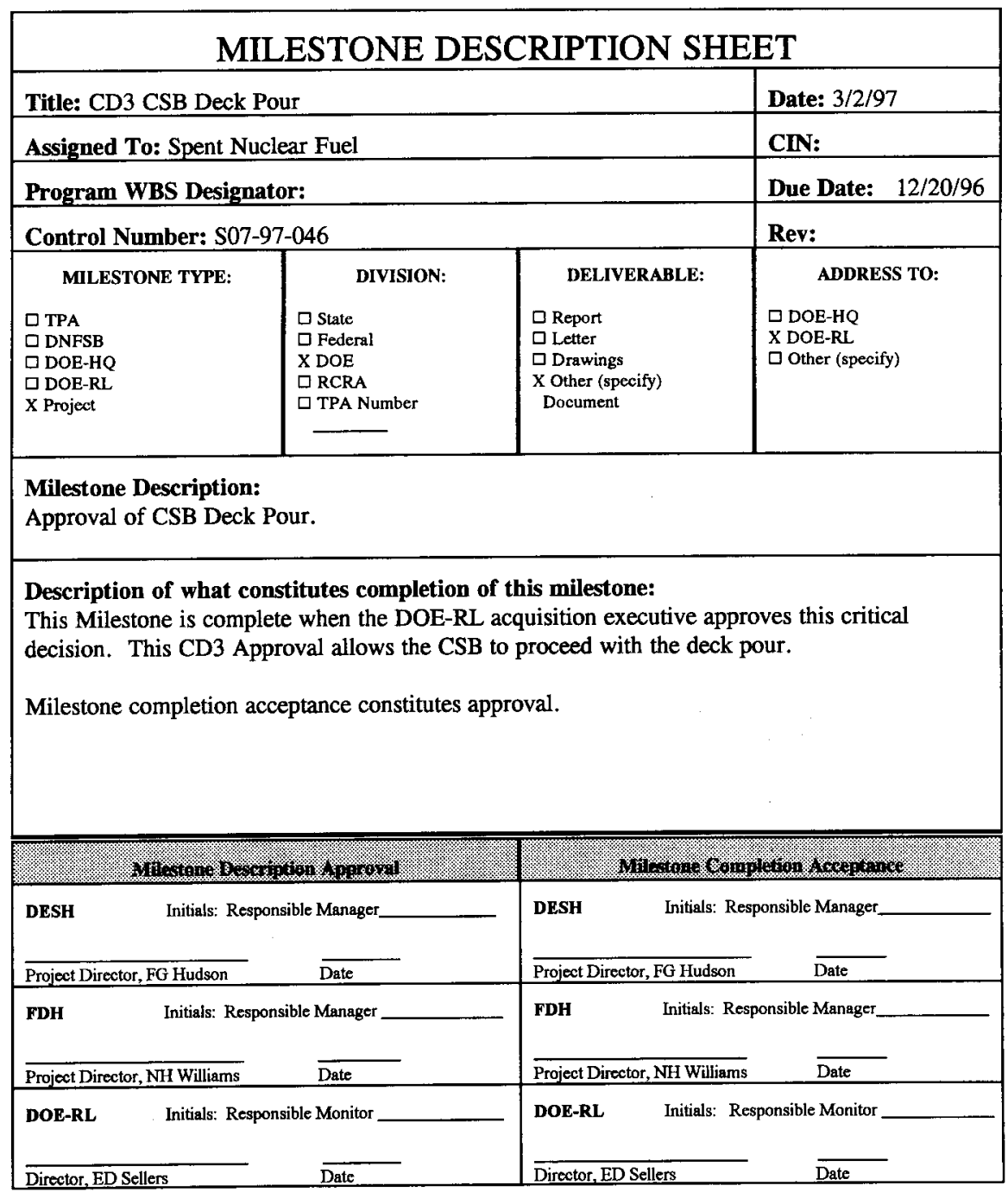




\section{SPENT NUCLEAR FUEL PROJECT \\ WBS 1.4.1}

HNF-SP-1 104, Rev 4

\section{Westinghouse Hanford Company \\ MILESTONE DESCRIPTION SHEET}

\begin{tabular}{|c|c|c|c|}
\hline \multicolumn{3}{|c|}{$\begin{array}{l}\text { Title: Critical Decision (CD-3) to proceed with } \\
\text { Cask/Transportation System Fabrication. }\end{array}$} & Date: $1 / 31 / 96$ \\
\hline \multicolumn{3}{|c|}{ Assigned To: Spent Nuclear Fuel Project } & CIN: SNF-96-013 \\
\hline \multicolumn{3}{|c|}{ Program WBS Designator: 1.4.1.06.01.02.01.02 } & Due Date: $1 / 22 / 97$ \\
\hline \multicolumn{3}{|c|}{ Control Number: S06-97-011 } & Rev: 0 \\
\hline MILESTONE TYPE: & DIVISION: & DELIVERABLE: & ADDRESS TO: \\
\hline $\begin{array}{l}\square \text { DOE-HQ } \\
X \text { DOE-RL } \\
\square \text { CONTRACTOR }\end{array}$ & $\begin{array}{l}\square \text { State } \\
\square \text { Federal } \\
\times \text { DOE } \\
\square \text { RCRA } \\
\square \text { TPA Number }\end{array}$ & $\begin{array}{l}\square \text { Report } \\
\square \text { Letter } \\
\square \text { Drawings } \\
\text { X Other (specify) } \\
\text { CD-3 Presentation } \\
\text { Materials }\end{array}$ & $\begin{array}{l}\square \text { DOE-HQ } \\
X \text { DOE-RL } \\
\square \text { other (specify) }\end{array}$ \\
\hline
\end{tabular}

Milestone Description: Prepare Critical Decision presentation materials in accordance with DOE guidel ines, conduct the CD-3 presentation (if necessary).

Description of what constitutes completion of this milestone: This milestone is complete when WHC delivers the documentation necessary for a Critical Decision presentation to the acquisition executive in accordance with the draft guidance for performing CD's. Critical Decision is required by January 23, 1990 in order to maintain schedule objectives of the Cask/Transportation system. Long lead procurement activities will be initiated in advance of fabrication. However the long lead procurement will only be initiated with DOE-RL approval.

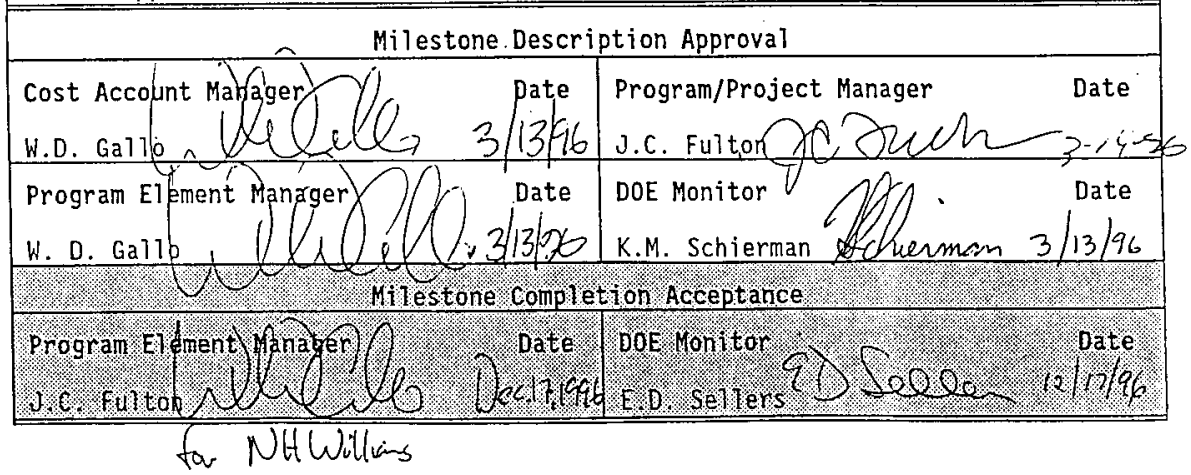




\section{MILESTONE DESCRIPTION SHEET}

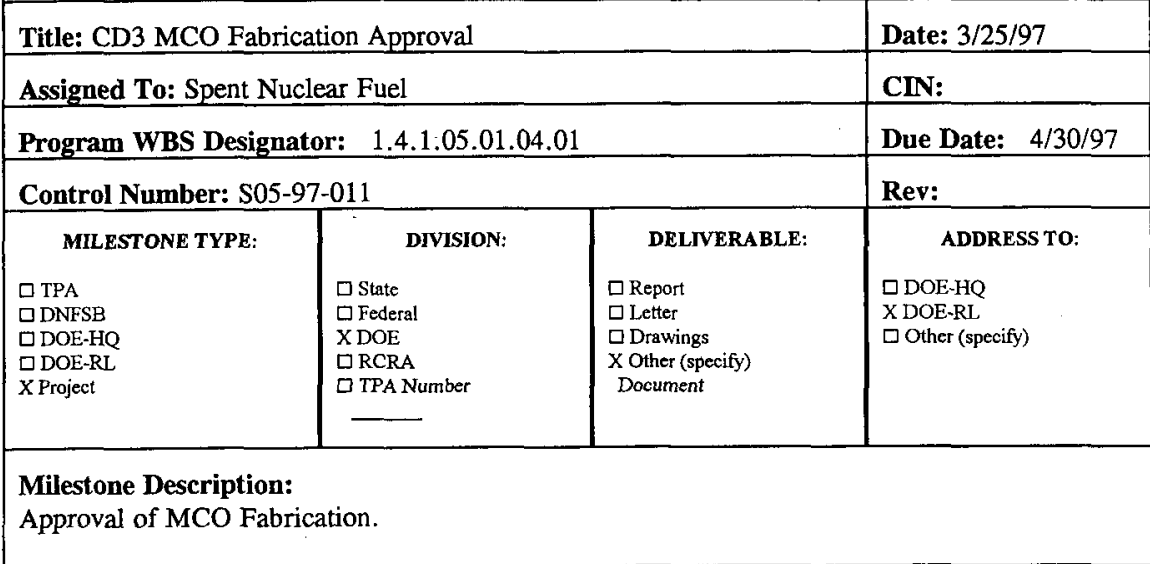

Description of what constitutes completion of this milestone:

This Milestone is complete when the DOE-RL acquisition executive approves this critical decision. This CD3 Approval allows the MCO to proceed with fabrication.

Milestone completion acceptance constitutes approval.

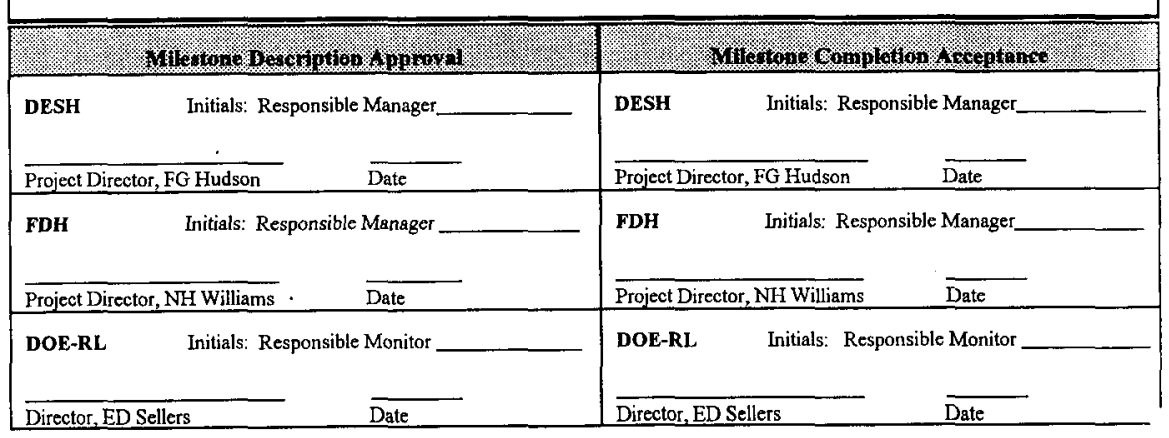




\section{SPENT NUCLEAR FUEL PROJECT \\ WBS 1.4.1}

HNF-SP-1104, Rev 4

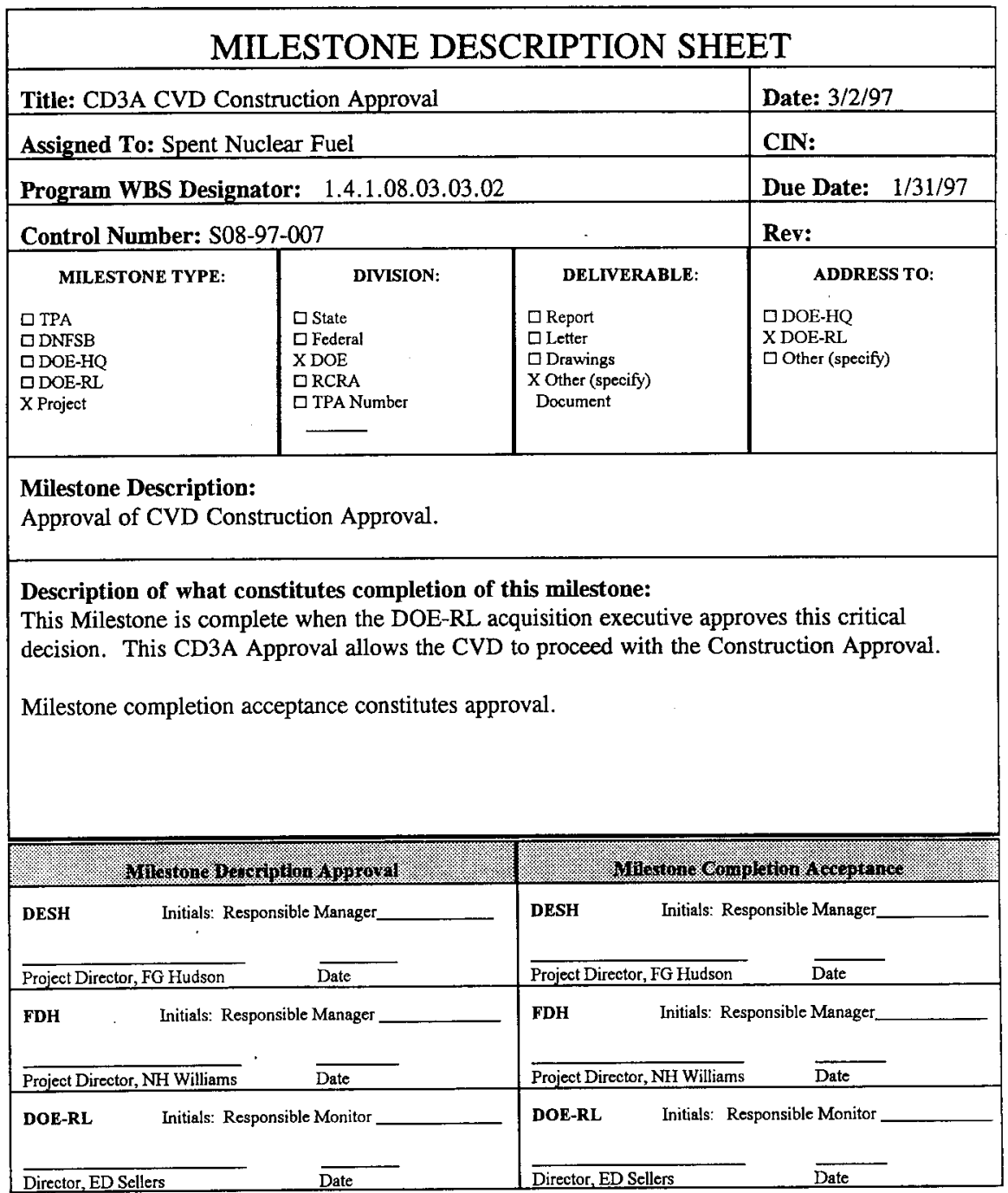




\section{Westinghouse Hanford Company MILESTONE DESCRIPTION SHEET}

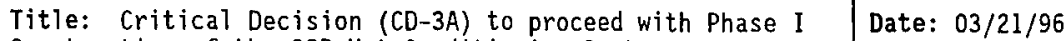
Construction of the CSB-Hot Conditioning System.

Assigned To: Spent Nuclear Fuel Project

Program WBS Designator: 1.4.1.08.01.03

Control Number: S08-97-012

MILESTONE TYPE:

DIVISION:

$\square$ DOE-HO

$X$ DOE-RL

$\square$ CONTRACTOR

DIVISION
$\square$ State
$\square$ Federal
$X$ DOE
$\square$ RCRA
$\square$ TPA Number

Milestone Description: Prepare Critical Decision presentation materials in accordance with DOE guidelines and conduct the CD-3A presentation (if necessary).

Description of what constitutes completion of this milestone: This milestone is complete when WHC delivers the documentation necessary for a Critical Decision presentation to the acquisition executive in accordance with the DOERL SFD guidance for performing CD's. Critical Decision is required by October 4,1996 in order to maintain schedule objectives of the CSB-Hot Conditioning system Sub-project.

\begin{tabular}{|c|c|c|}
\hline \multicolumn{3}{|c|}{ Milestone Description Approval } \\
\hline Cost Account Manager Bradshaw Date & $\begin{array}{l}\text { Program/Project Manager } \\
\text { Da a. Fultom f.u }\end{array}$ & $\begin{array}{c}\text { Date } \\
7-25-96\end{array}$ \\
\hline Program Element Managen Gallo Date & $\begin{array}{l}\text { DoE Monjtor } \\
\text { E. Sryson sel tor } \\
\text { E. Sellers }\end{array}$ & Date \\
\hline Milestone Compliet & 1on Acceptance. & \\
\hline 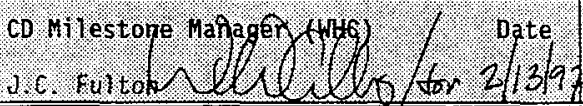 & co y liestone ranager (pot) & 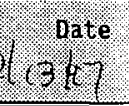 \\
\hline
\end{tabular}




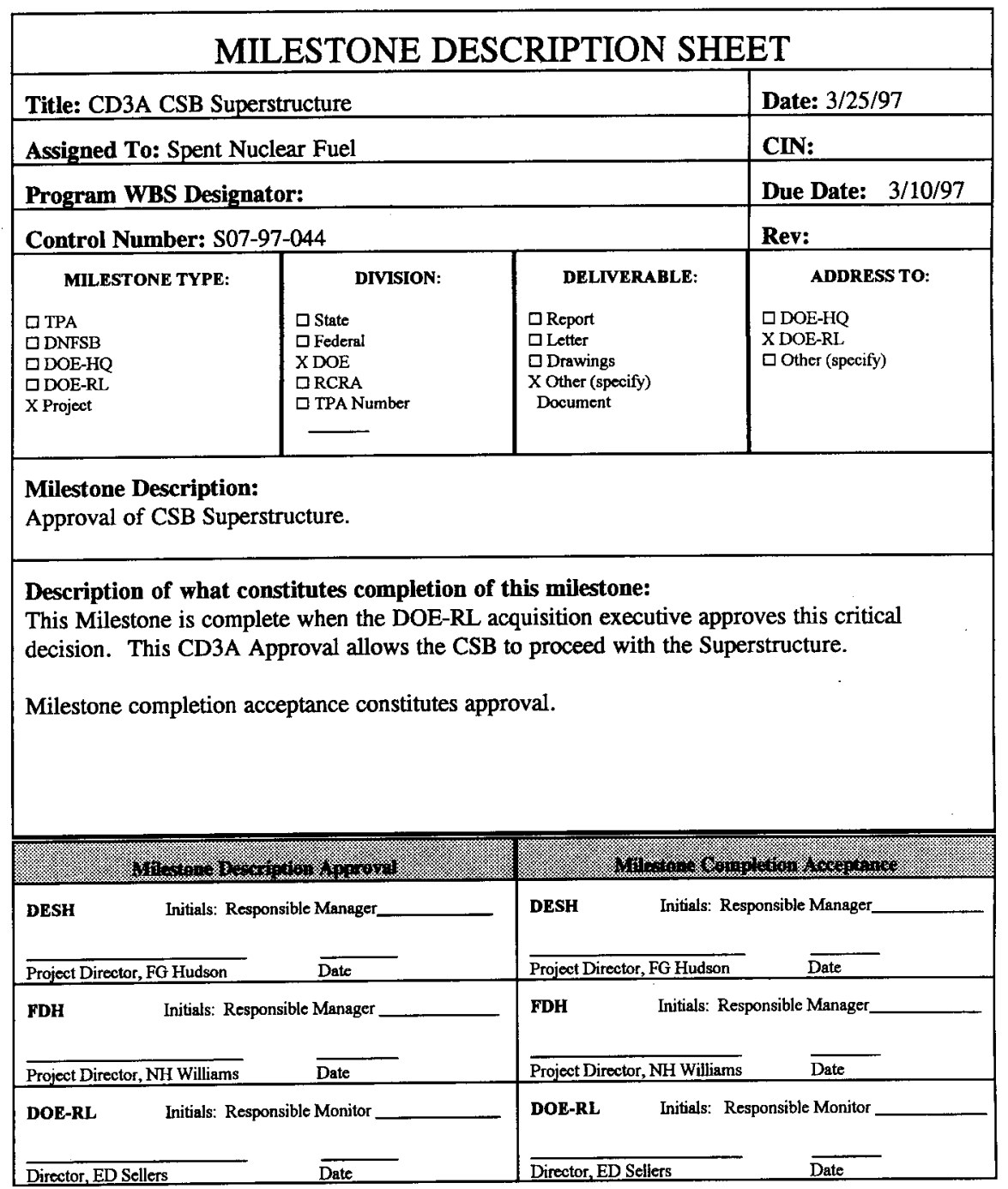




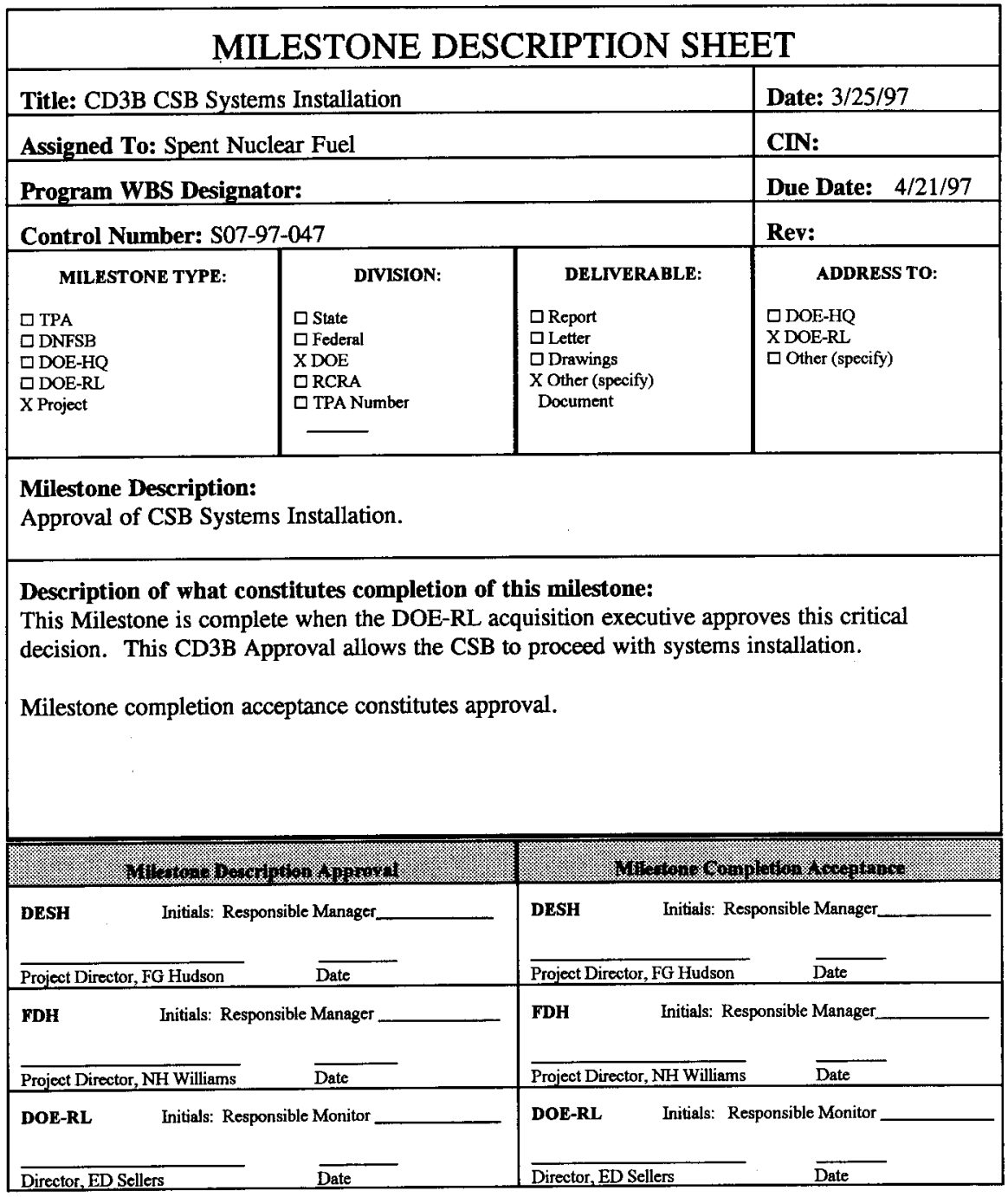




\section{MILESTONE DESCRIPTION SHEET}

\begin{tabular}{|c|c|c|c|}
\hline \multicolumn{3}{|c|}{ Title: CD3B HCS Process Equipment Procurement Approval } & Date: $3 / 25 / 97$ \\
\hline \multicolumn{3}{|c|}{ Assigned To: Spent Nuclear Fuel } & CIN: \\
\hline \multicolumn{3}{|c|}{ Program WBS Designator: } & Due Date: $4 / 30 / 97$ \\
\hline \multicolumn{3}{|c|}{ Control Number: S08-97-008 } & Rev: \\
\hline $\begin{array}{l}\quad \text { MILESTONE TYPE: } \\
\text { 口 TPA } \\
\text { 口DNFSB } \\
\text { 口DOE-HQ } \\
\text { व DOE-RL } \\
\text { X Project }\end{array}$ & $\begin{array}{l}\quad \text { DIVISION: } \\
\text { a State } \\
\square \text { Federal } \\
\text { X DOE } \\
\text { व RCRA } \\
\square \text { TPA Number }\end{array}$ & $\begin{array}{l}\quad \text { DELIVERABLE: } \\
\text { वReport } \\
\text { 口Letter } \\
\text { Q Drawings } \\
\text { X Other (specify) } \\
\text { Document }\end{array}$ & $\begin{array}{l}\quad \text { ADDRESS TO: } \\
\text { Q DOE-HQ } \\
\text { X DOE-RL } \\
\text { 口 Other (specify) }\end{array}$ \\
\hline
\end{tabular}

\section{Milestone Description:}

Approval of HCS Process Procurement.

\section{Description of what constitutes completion of this milestone:}

This Milestone is complete when the DOE-RL acquisition executive approves this critical decision. This CD3B Approval allows the HCS to proceed with procurement.

Milestone completion acceptance constitutes approval.

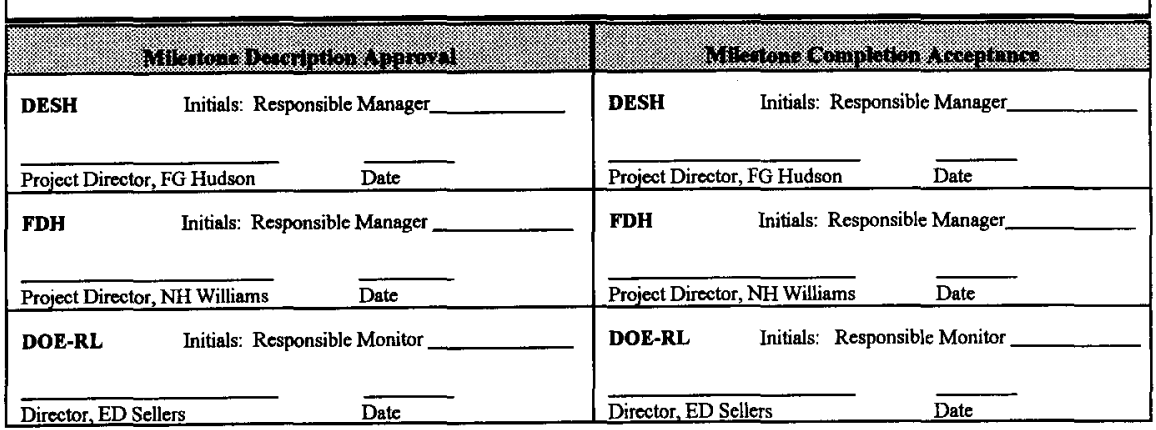




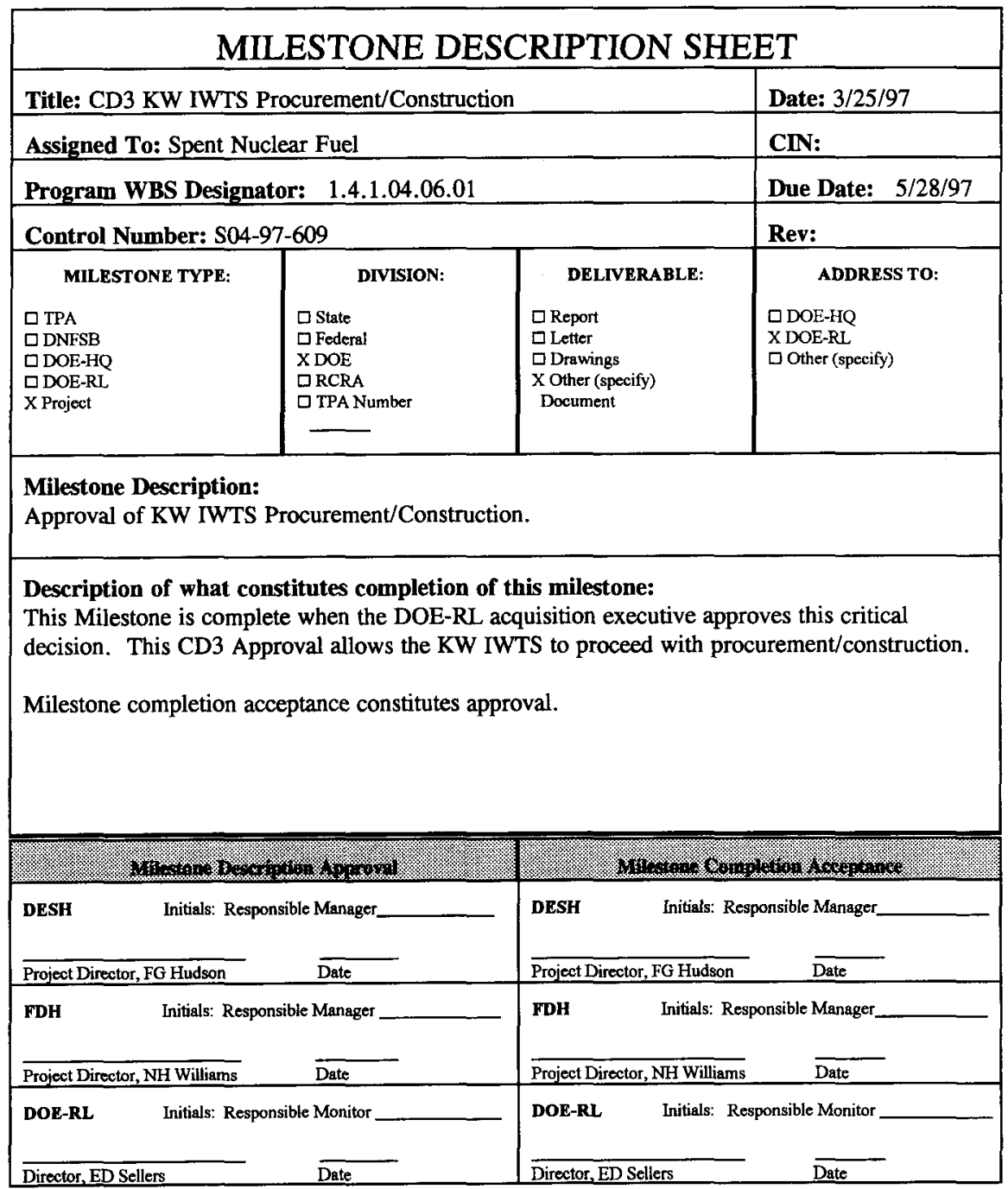




\section{MILESTONE DESCRIPTION SHEET}

\begin{tabular}{|c|c|c|c|}
\hline \multicolumn{3}{|c|}{ Title: CD3C CSB MHM Installation } & Date: $3 / 25 / 97$ \\
\hline \multicolumn{3}{|c|}{ Assigned To: Spent Nuclear Fuel } & CIN: \\
\hline \multicolumn{3}{|c|}{ Program WBS Designator: } & Due Date: $5 / 30 / 97$ \\
\hline \multicolumn{3}{|c|}{ Control Number: S07-97-048 } & Rev: \\
\hline $\begin{array}{l}\quad \text { MILESTONE TYPE: } \\
\text { 口 TPA } \\
\text { Q DNFSB } \\
\text { Q DOE-HQ } \\
\text { व DOE-RL } \\
\text { X Project }\end{array}$ & $\begin{array}{l}\quad \text { DIVISION: } \\
\text { ㅁ State } \\
\text { ㅁ Federal } \\
\text { X DOE } \\
\text { 口 RCRA } \\
\text { 口 TPA Number }\end{array}$ & $\begin{array}{l}\quad \text { DELIVERABLE: } \\
\text { a Report } \\
\text { aLefter } \\
\text { a Drawings } \\
\text { X Other (specify) } \\
\text { Document }\end{array}$ & $\begin{array}{l}\quad \text { ADDRESS TO: } \\
\text { 口 DOE-HQ } \\
\text { X DOE-RL } \\
\text { 口 Other (specify) }\end{array}$ \\
\hline
\end{tabular}

\section{Milestone Description:}

Approval of CSB MHM Installation.

Description of what constitutes completion of this milestone:

This Milestone is complete when the DOE-RL acquisition executive approves this critical decision. This CD3C Approval allows the CSB to proceed with MHM installation.

Milestone completion acceptance constitutes approval.

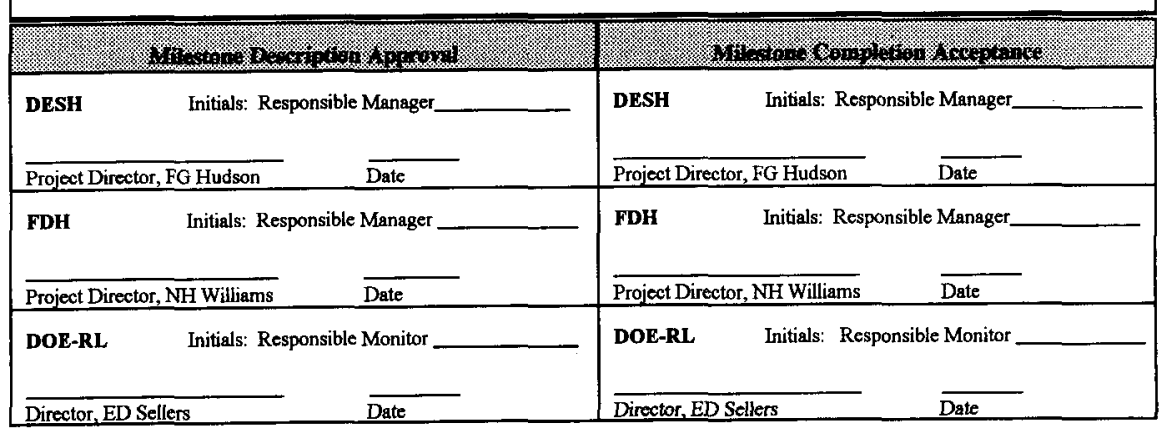




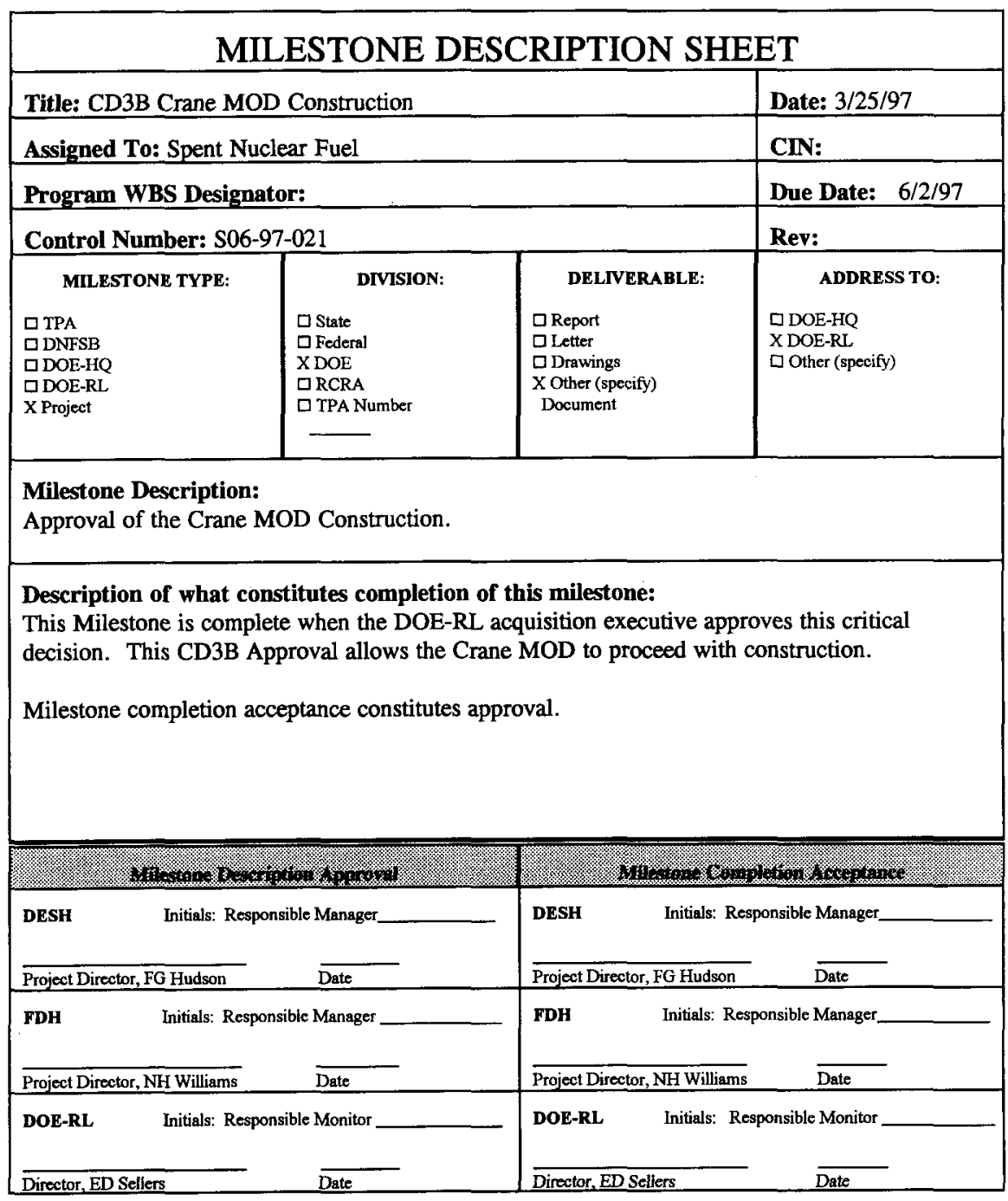




\section{MILESTONE DESCRIPTION SHEET}

Title: CD3B CVD Equipment Installation Approval Date: $3 / 2 / 97$

Assigned To: Spent Nuclear Fuel CIN:

Program WBS Designator: Due Date: 6/27/97

Control Number: S08-97-020 MILESTONE TYPE:

\begin{tabular}{l|l|l}
\multicolumn{1}{c|}{ MILESTONE TYPE: } & \multicolumn{1}{|c}{ DIVISION: } & \multicolumn{1}{c}{ DELIVERABLE: } \\
$\square$ TPA & $\square$ State & $\square$ Report \\
$\square$ DNFSB & $\square$ Federal & $\square$ Letter \\
$\square$ DOE-HQ & X DOE & $\square$ Drawings \\
$\square$ DOE-RL & $\square$ RCRA & X Other (specify) \\
X Project & $\square$ TPA Number & \\
& & \\
& & \\
& &
\end{tabular}
Rev:

\section{Milestone Description:}

Approval of CVD Equipment Installation Approval.

\section{Description of what constitutes completion of this milestone:}

This Milestone is complete when the DOE-RL acquisition executive approves this critical decision. This CD3B Approval allows the CVD to proceed with the equipment installation approval.

Milestone completion acceptance constitutes approval.

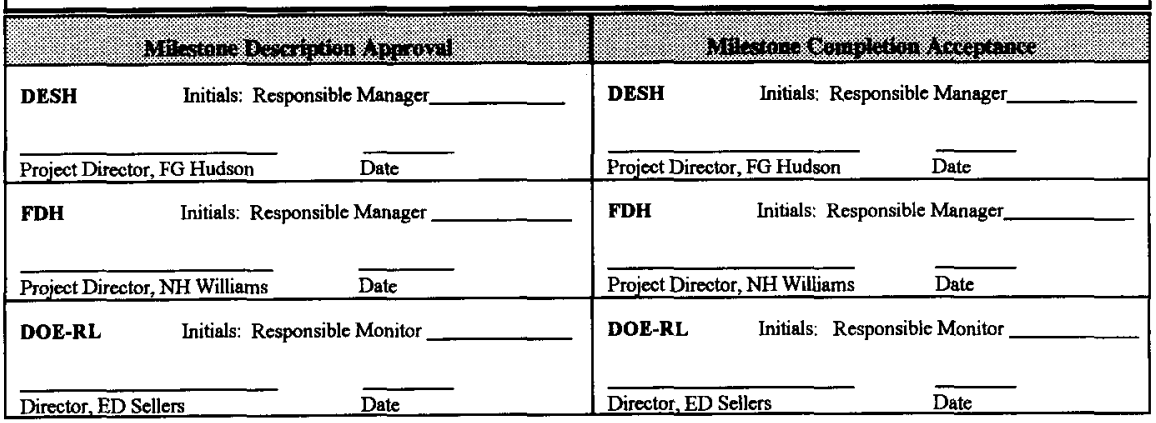




\section{SPENT NUCLEAR FUEL PROJECT \\ WBS 1.4.1}

HNF-SP-1104, Rev 4

\section{MILESTONE DESCRIPTION SHEET}

\begin{tabular}{|c|c|c|c|}
\hline \multicolumn{3}{|c|}{ Title: CD3 KE IWTS Procurement/Construction } & Date: $3 / 25 / 97$ \\
\hline \multicolumn{3}{|c|}{ Assigned To: Spent Nuclear Fuel } & CIN: \\
\hline \multicolumn{3}{|c|}{ Program WBS Designator: 1.4 .1 .04 .06 .01} & Due Date: $7 / 15 / 97$ \\
\hline \multicolumn{3}{|c|}{ Control Number: S04-97-616 } & Rev: \\
\hline $\begin{array}{l}\quad \text { MILESTONE TYPE: } \\
\square \text { TPA } \\
\square \text { DNFSB } \\
\square \text { DOE-HQ } \\
\square \text { DOE-RL } \\
\text { X Project }\end{array}$ & $\begin{array}{l}\quad \text { DIVISION: } \\
\square \text { State } \\
\square \text { Federal } \\
\text { X DOE } \\
\square \text { RCRA } \\
\square \text { TPA Number }\end{array}$ & $\begin{array}{l}\quad \text { DELIVERABLE: } \\
\text { 口 Report } \\
\square \text { Letter } \\
\square \text { Drawings } \\
\text { X Other (specify) } \\
\text { Document }\end{array}$ & $\begin{array}{l}\quad \text { ADDRESS TO: } \\
\text { a DOE-HQ } \\
\text { X DOE-RL } \\
\square \text { Other (specify) }\end{array}$ \\
\hline
\end{tabular}

\section{Milestone Description:}

Approval of KE IWTS Procurement/Construction.

\section{Description of what constitutes completion of this milestone:}

This Milestone is complete when the DOE-RL acquisition executive approves this critical decision. This CD3 Approval allows the KE IWTS to proceed with procurement/Construction.

Milestone completion acceptance constitutes approval.

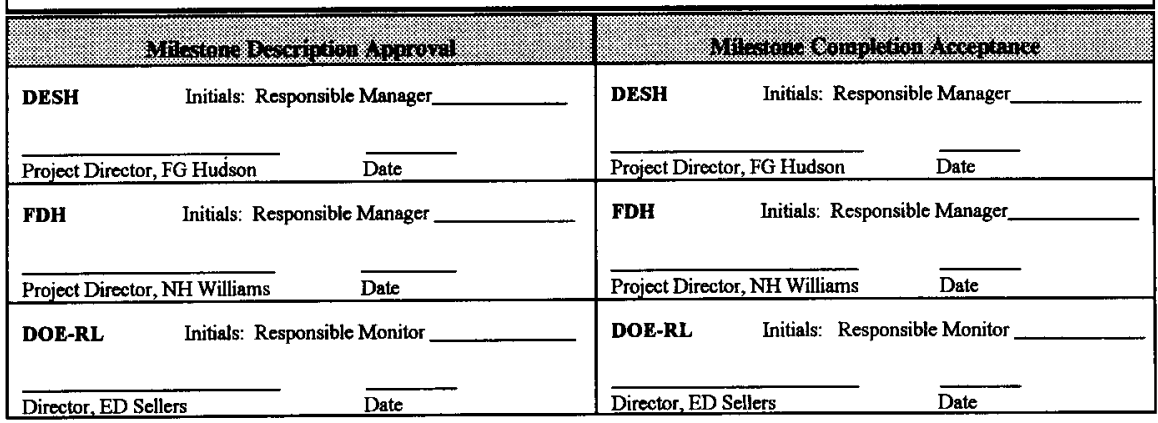




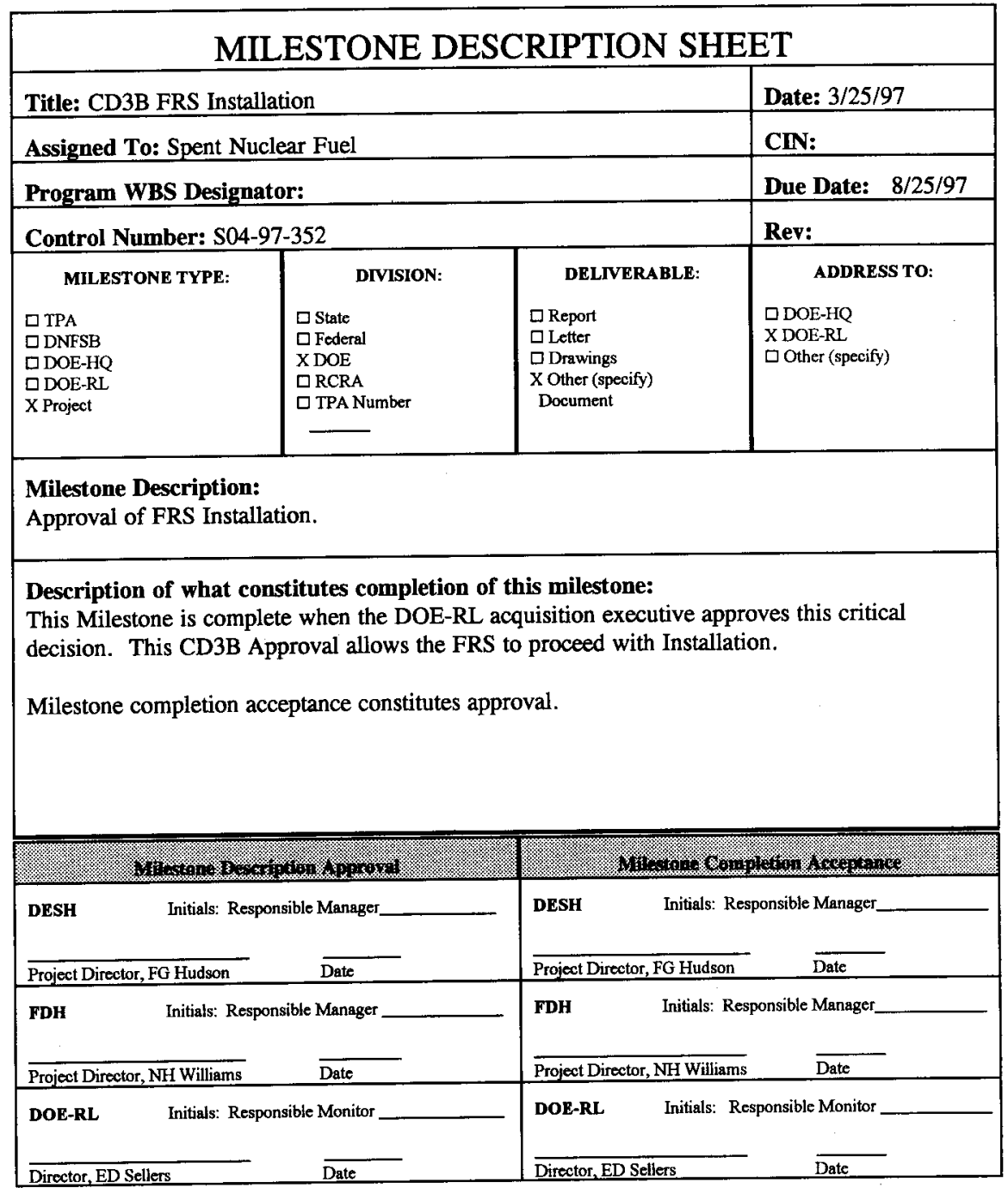




\section{MILESTONE DESCRIPTION SHEET}

\begin{tabular}{|c|c|c|c|}
\hline \multicolumn{3}{|c|}{ Title: CD2/3 PWS Definitive Design \& Procurement/Construction } & Date: $3 / 25 / 97$ \\
\hline \multicolumn{3}{|c|}{ Assigned To: Spent Nuclear Fuel } & CIN: \\
\hline \multicolumn{3}{|c|}{ Program WBS Designator: 1.4 .1 .04 .03 .07 .01 .02} & Due Date: $10 / 31 / 97$ \\
\hline \multicolumn{3}{|c|}{ Control Number:S04-98-105 } & Rev: \\
\hline $\begin{aligned} & \text { MILESTONE TYPE: } \\
& \square \text { TPA } \\
& \square \text { DNFSB } \\
& \square \text { DOE-HQ } \\
& \square \text { DOE-RL } \\
& \text { X Project }\end{aligned}$ & $\begin{array}{l}\quad \text { DIVISION: } \\
\quad \square \text { State } \\
\square \text { Federal } \\
\text { X DOE } \\
\square \text { RCRA } \\
\square \text { TPA Number }\end{array}$ & $\begin{array}{l}\text { DELIVERABLE: } \\
\text { QReport } \\
\square \text { Letter } \\
\square \text { Drawings } \\
\text { X Other (specify) } \\
\text { Document }\end{array}$ & $\begin{array}{l}\quad \text { ADDRESS TO: } \\
\text { } \square \text { DOE-HQ } \\
\text { X DOE-RL } \\
\square \text { Other (specify) }\end{array}$ \\
\hline
\end{tabular}

\section{Milestone Description:}

Approval of PWS Definitive Design \& Procurement /Construction.

\section{Description of what constitutes completion of this milestone:}

This Milestone is complete when the DOE-RL acquisition executive approves this critical decision. This CD2/3 Approval allows the PWS to proceed with Definitive Design \& Procurement Construction.

Milestone completion acceptance constitutes approval.

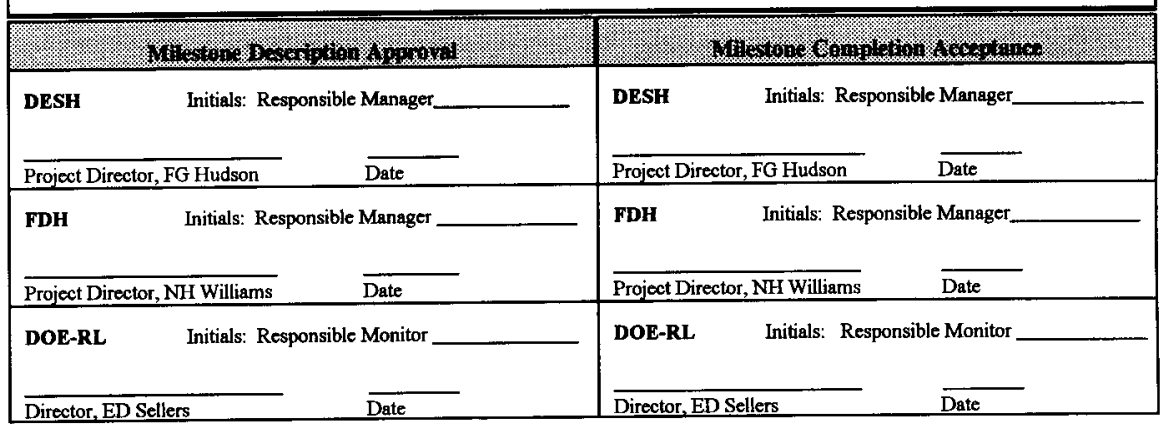




\section{SPENT NUCLEAR FUEL PROJECT \\ WBS 1.4.1}

HNF-SP-1 104, Rev 4

\section{MILESTONE DESCRIPTION SHEET}

\begin{tabular}{|c|c|c|c|}
\hline \multicolumn{3}{|c|}{ Title: CD3C HCS Process Equipment Installation } & Date: $3 / 25 / 97$ \\
\hline \multicolumn{3}{|c|}{ Assigned To: Spent Nuclear Fuel } & CIN: \\
\hline \multicolumn{3}{|c|}{ Program WBS Designator: 1.4 .1 .08 .01 .03 .02} & Due Date: $11 / 14 / 97$ \\
\hline \multicolumn{3}{|c|}{ Control Number: S08-98-025 } & Rev: \\
\hline $\begin{array}{l}\quad \text { MILESTONE TYPE: } \\
\square \text { TPA } \\
\text { 口DNFSB } \\
\square \text { DOE-HQ } \\
\square \text { DOE-RL } \\
\text { X Project }\end{array}$ & $\begin{array}{l}\quad \text { DIVISION: } \\
\text { 口 State } \\
\square \text { Federal } \\
\text { XDOE } \\
\square \text { RCRA } \\
\square \text { TPA Number }\end{array}$ & $\begin{array}{l}\quad \text { DELIVERABLE: } \\
\square \text { Report } \\
\square \text { Letter } \\
\square \text { Drawings } \\
\text { X Other (specify) } \\
\text { Document }\end{array}$ & $\begin{array}{l}\quad \text { ADDRESS TO: } \\
\text { 口 DOE-HQ } \\
\text { X DOE-RL } \\
\text { 口 Other (specify) }\end{array}$ \\
\hline
\end{tabular}

\section{Milestone Description:}

Approval of HCS Process Equipment Installation.

\section{Description of what constitutes completion of this milestone:}

This Milestone is complete when the DOE-RL acquisition executive approves this critical decision. This CD3C Approval allows the HCS to proceed with equipment installation.

Milestone completion acceptance constitutes approval.

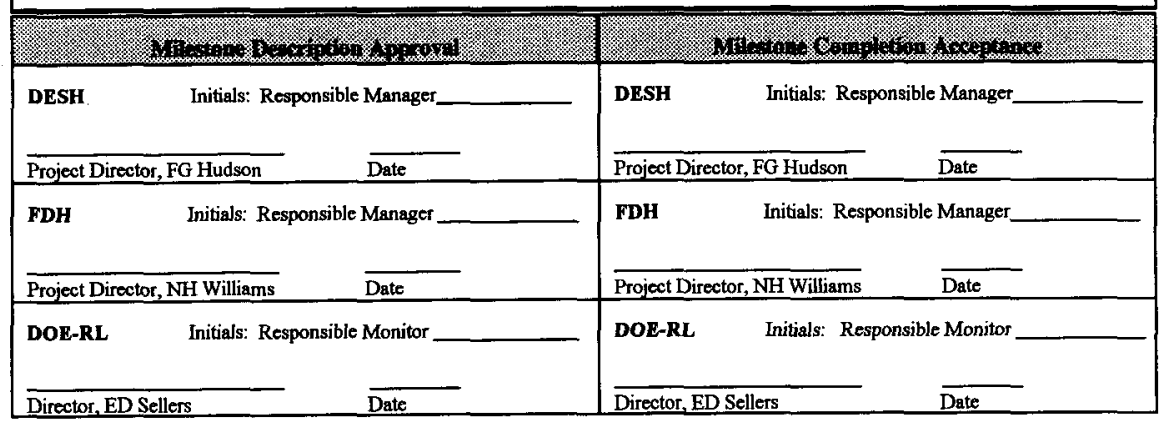




\section{SPENT NUCLEAR FUEL PROJECT \\ WBS 1.4.1}

HNF-SP-1 104, Rev 4

\section{MILESTONE DESCRIPTION SHEET}

\begin{tabular}{|c|c|c|c|}
\hline \multicolumn{3}{|c|}{ Title: CD4 KW IWTS Operations } & Date: $3 / 25 / 97$ \\
\hline \multicolumn{3}{|c|}{ Assigned To: Spent Nuclear Fuel } & CIN: \\
\hline \multicolumn{3}{|c|}{ Program WBS Designator: 1.4 .1 .04 .06 .02} & Due Date: $2 / 26 / 98$ \\
\hline \multicolumn{3}{|c|}{ Control Number: S04-98-607 } & Rev: \\
\hline $\begin{array}{l}\quad \text { MILESTONE TYPE: } \\
\square \text { TPA } \\
\square \text { DNFSB } \\
\square \text { DOE-HQ } \\
\square \text { DOE-RL } \\
\text { X Project }\end{array}$ & $\begin{array}{l}\quad \text { DIVISION: } \\
\quad \square \text { State } \\
\square \text { Federal } \\
\text { X DOE } \\
\square \text { RCRA } \\
\square \text { TPA Number }\end{array}$ & $\begin{array}{l}\text { DELIVERABLE: } \\
\square \text { Report } \\
\square \text { Letter } \\
\square \text { Drawings } \\
\text { X Other (specify) } \\
\text { Document }\end{array}$ & $\begin{array}{l}\quad \text { ADDRESS TO: } \\
\text { } \square \text { DOE-HQ } \\
\text { X DOE-RL } \\
\square \text { Other (specify) }\end{array}$ \\
\hline
\end{tabular}

Milestone Description:

Approval of KW IWTS Operations.

Description of what constitutes completion of this milestone:

This Milestone is complete when the DOE-RL acquisition executive approves this critical decision. This CD4 Approval allows the KW IWTS to proceed with operations.

Milestone completion acceptance constitutes approval.

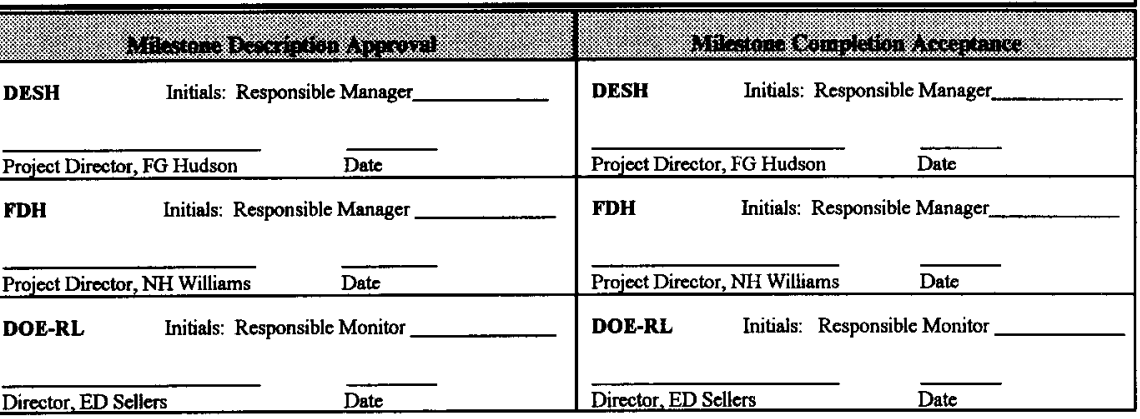




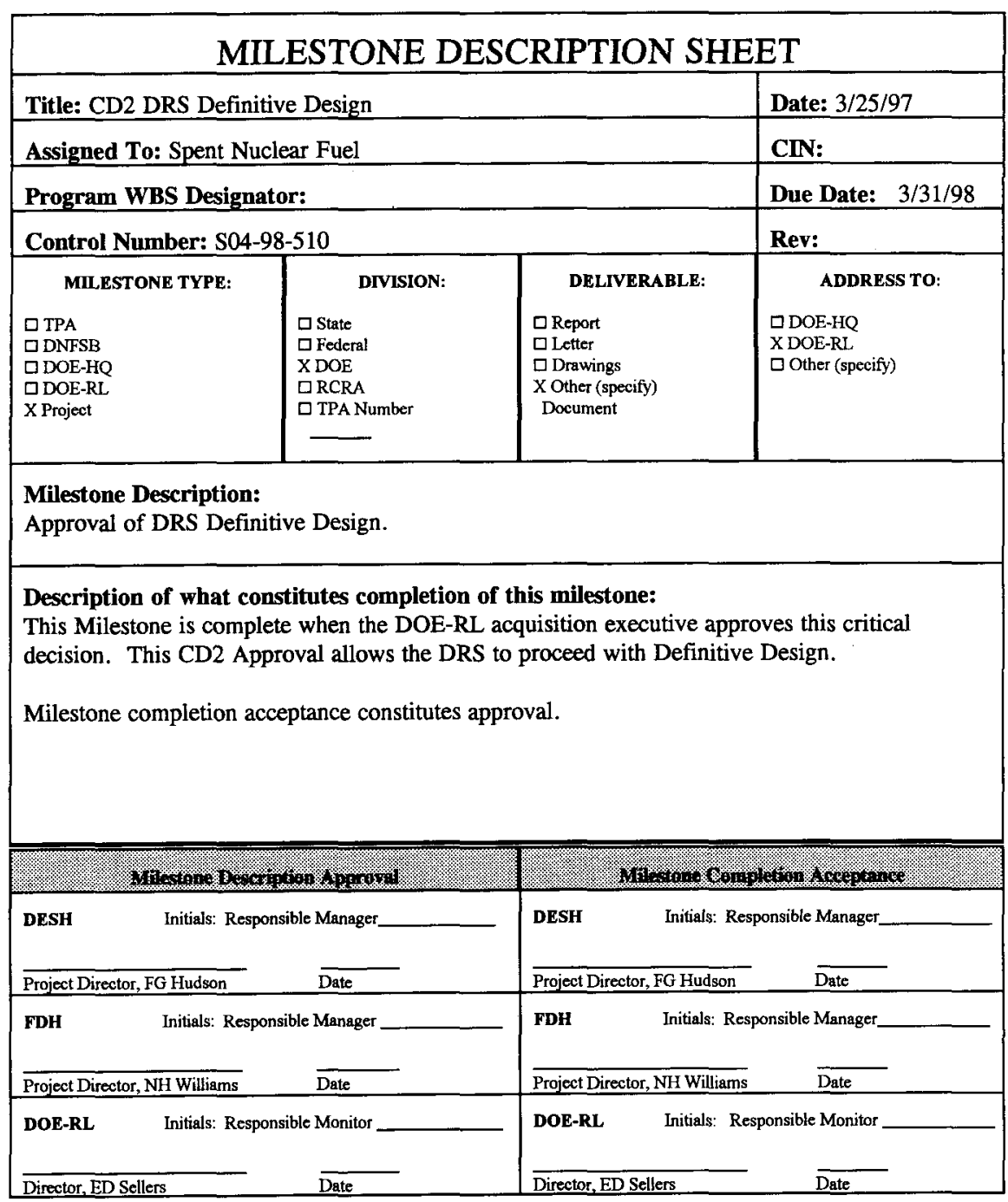




\section{MILESTONE DESCRIPTION SHEET}

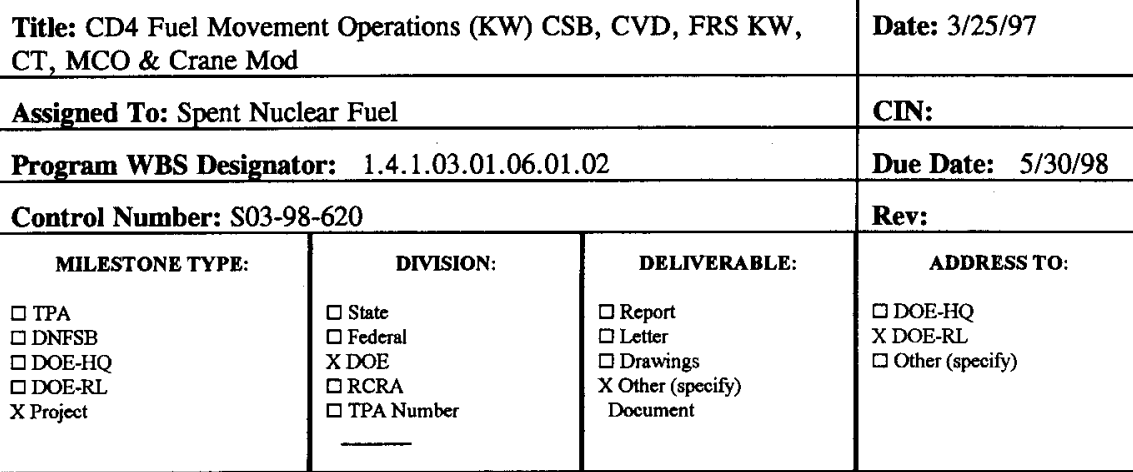

\section{Milestone Description:}

Approval of Fuel Movement Operations.

\section{Description of what constitutes completion of this milestone:}

This Milestone is complete when the DOE-RL acquisition executive approves this critical decision. This CD4 Approval allows the Fuel Movement to proceed with operations.

Milestone completion acceptance constitutes approval.

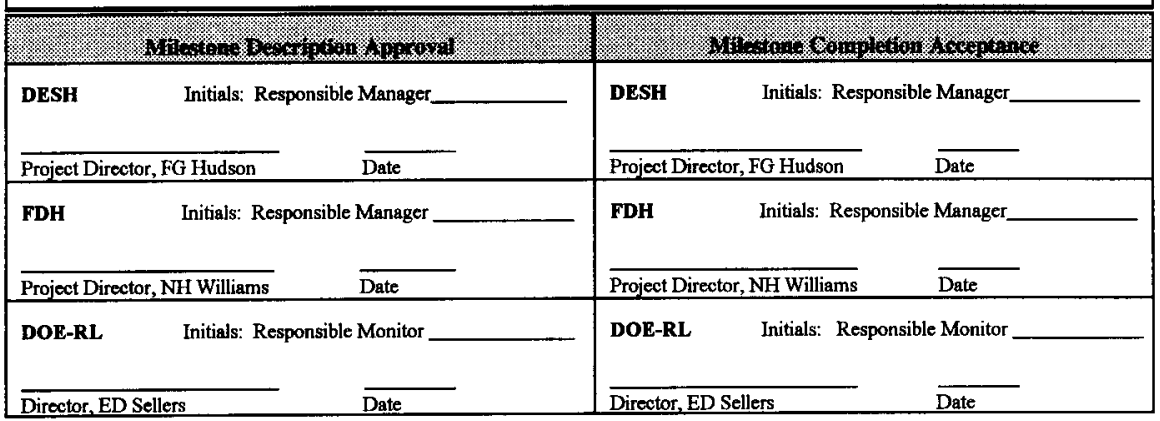




\section{MILESTONE DESCRIPTION SHEET}

Title: CD3 DRS Procurement/Construction

Date: $3 / 25 / 97$

Assigned To: Spent Nuclear Fuel CIN:

Program WBS Designator:

Control Number: S04-98-511

\begin{tabular}{|c|c|c|c|}
\hline $\begin{array}{l}\quad \text { MILESTONE TYPE: } \\
\square \text { TPA } \\
\square \text { DNESB } \\
\square \text { DOE-HQ } \\
\square \text { DOE-RL } \\
\text { X Project }\end{array}$ & $\begin{array}{l}\quad \text { DIVISION: } \\
\text { } \square \text { State } \\
\square \text { Federal } \\
\text { X DOE } \\
\square \text { RCRA } \\
\square \text { TPA Number }\end{array}$ & $\begin{array}{l}\quad \text { DELIVERABLE: } \\
\text { } \square \text { Report } \\
\square \text { Letter } \\
\square \text { Drawings } \\
\text { X Other (specify) } \\
\text { Document }\end{array}$ & $\begin{array}{l}\text { ADDRESS TO: } \\
\square \text { DOE-HQ } \\
\text { X DOE-RL } \\
\square \text { Other (specify) }\end{array}$ \\
\hline
\end{tabular}

\section{Milestone Description:}

Approval of DRS Procurement/Construction.

\section{Description of what constitutes completion of this milestone:}

This Milestone is complete when the DOE-RL acquisition executive approves this critical decision. This CD3 Approval allows the DRS to proceed with procurement/construction.

Milestone completion acceptance constitutes approval.

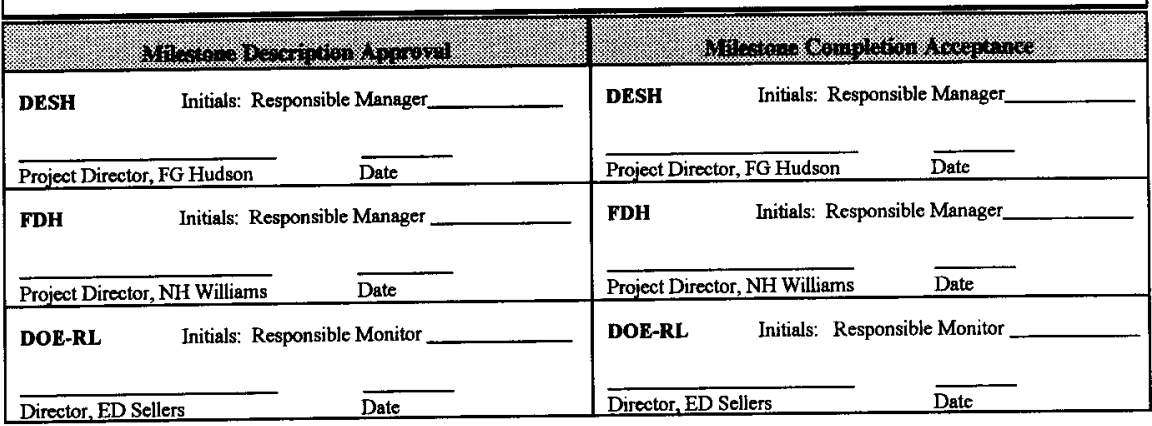




\section{SPENT NUCLEAR FUEL PROJECT \\ WBS 1.4.1}

HNF-SP-1 104, Rev 4

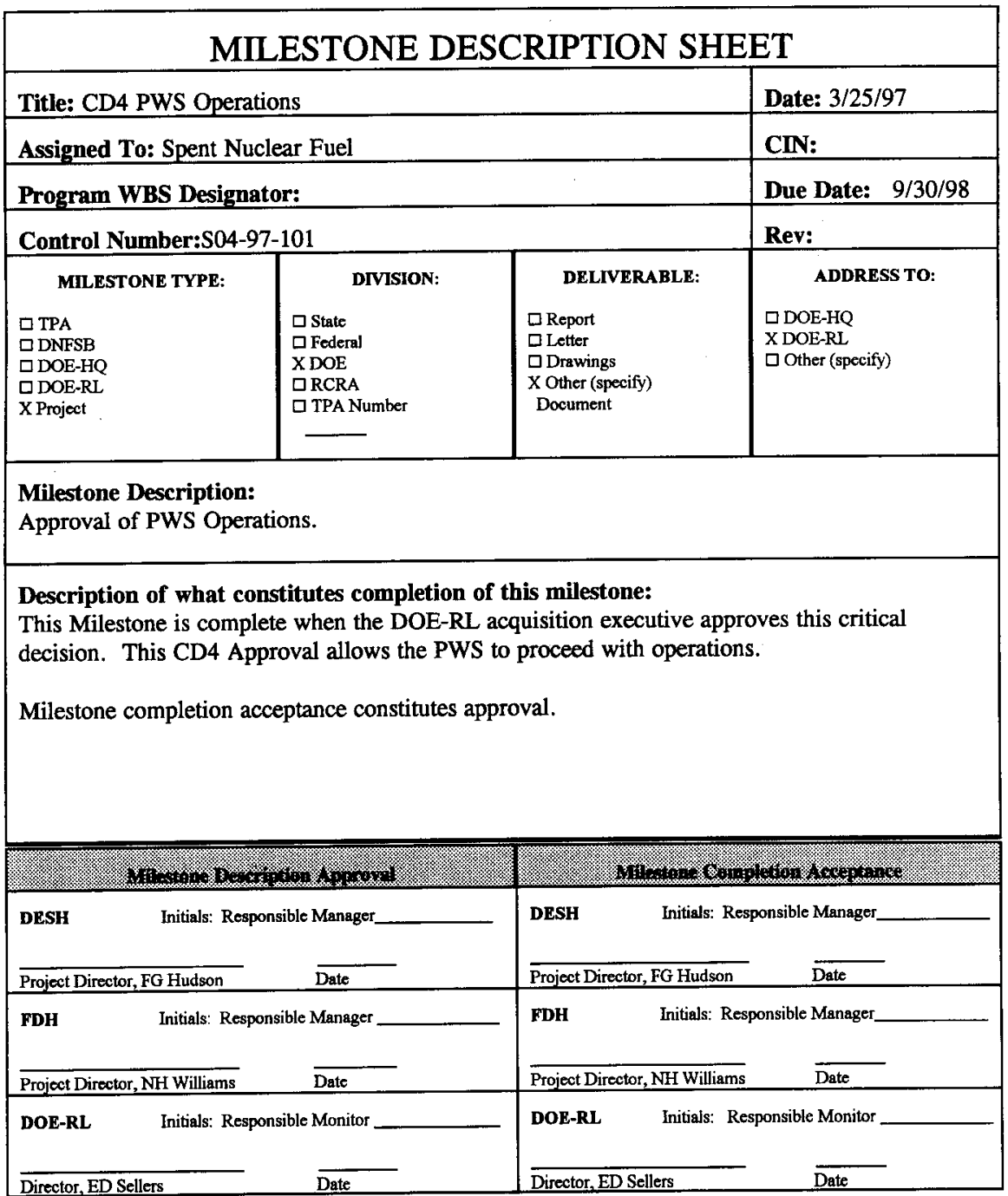




\section{SPENT NUCLEAR FUEL PROJECT \\ WBS 1.4.1}

HNF-SP-1 104, Rev 4

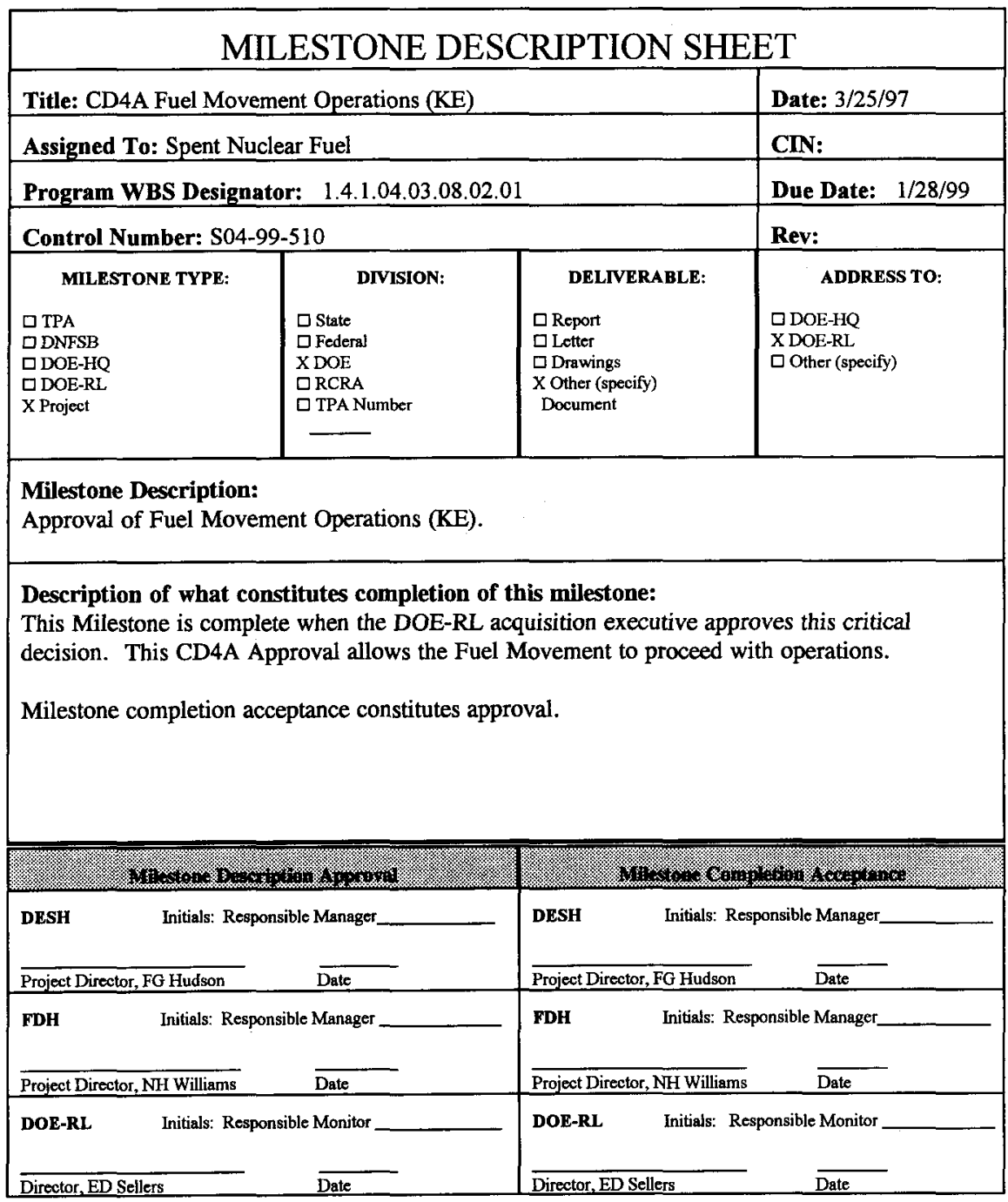




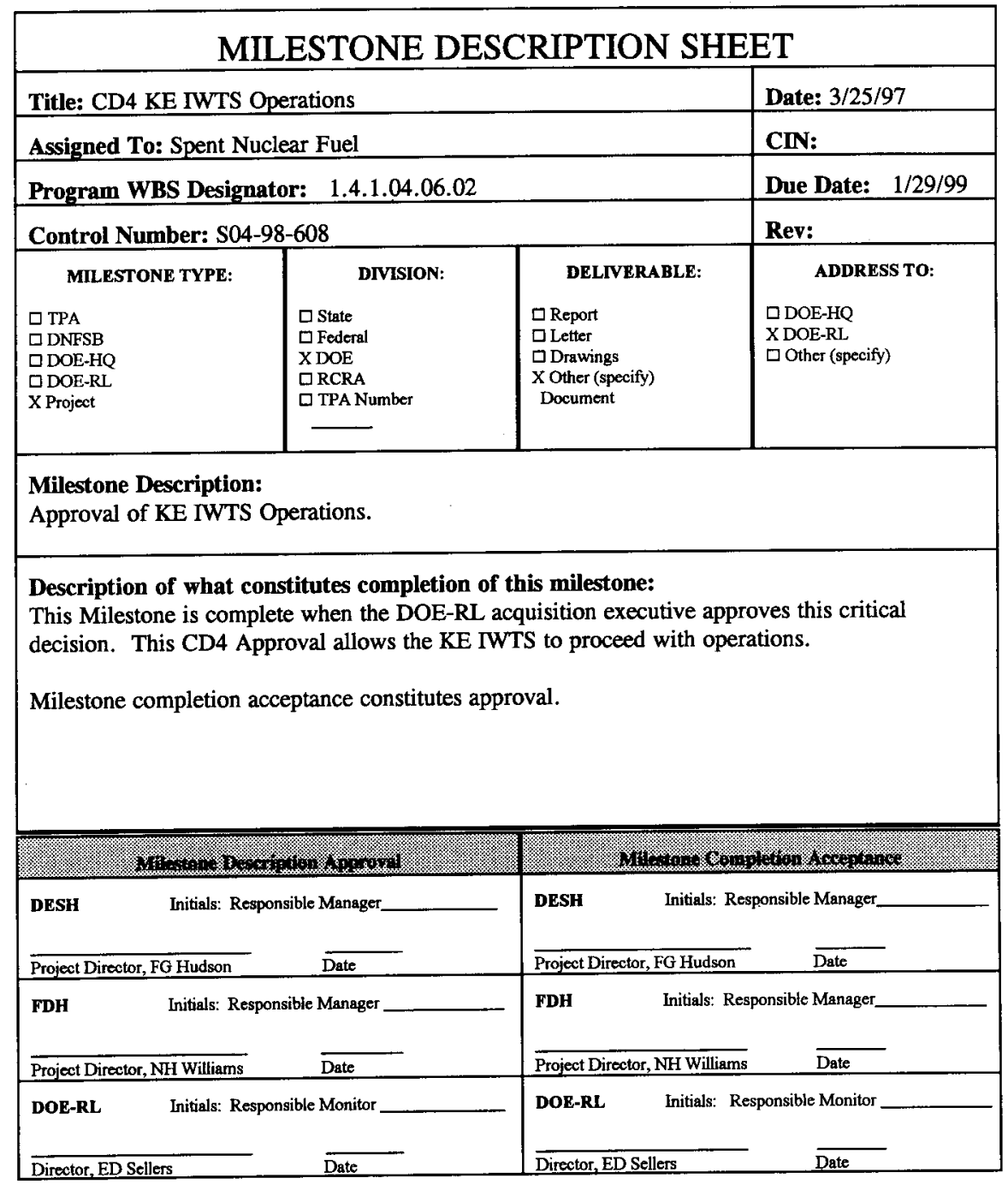




\section{SPENT NUCLEAR FUEL PROJECT \\ WBS 1.4.1}

HNF-SP-1 104, Rev 4

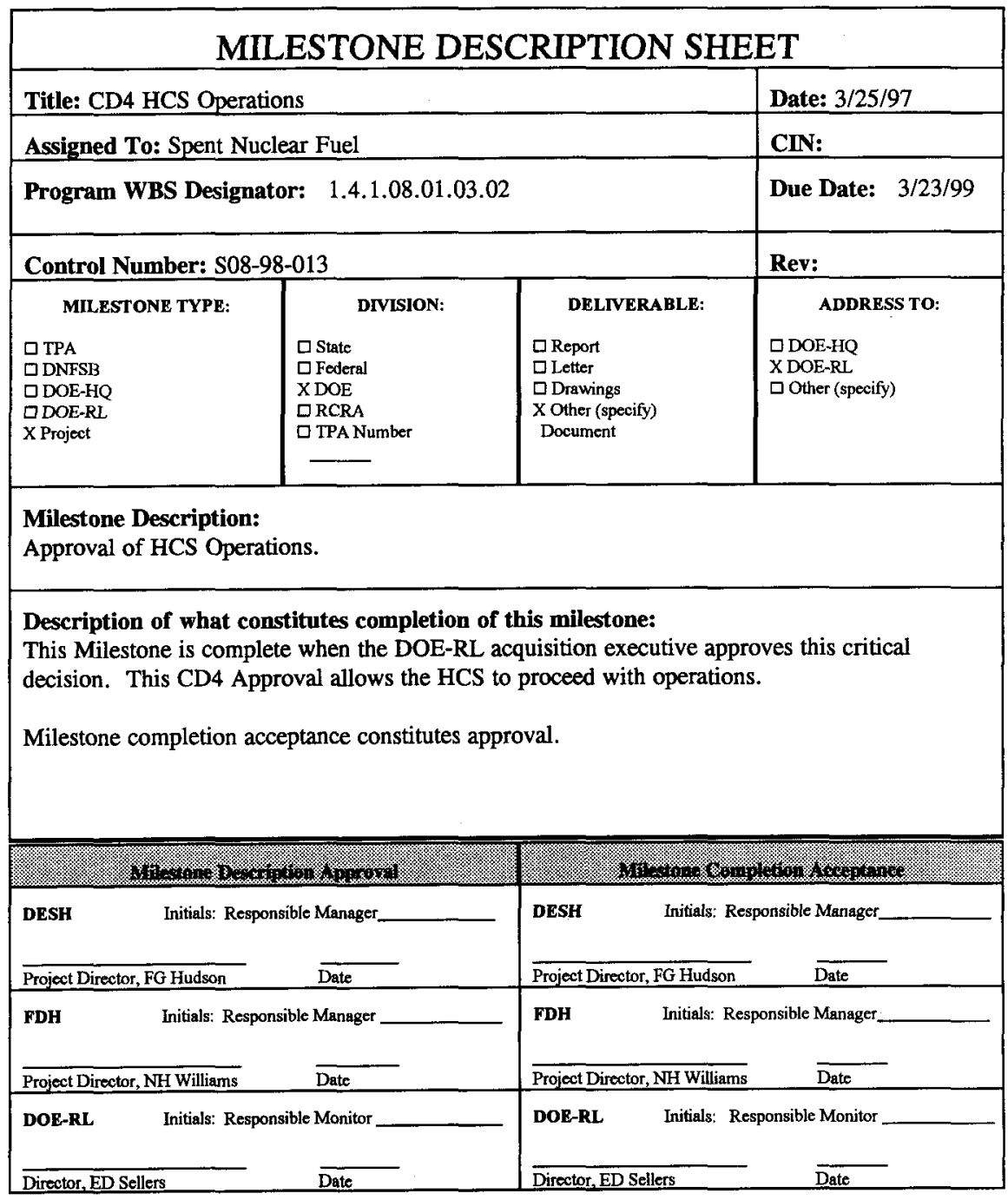




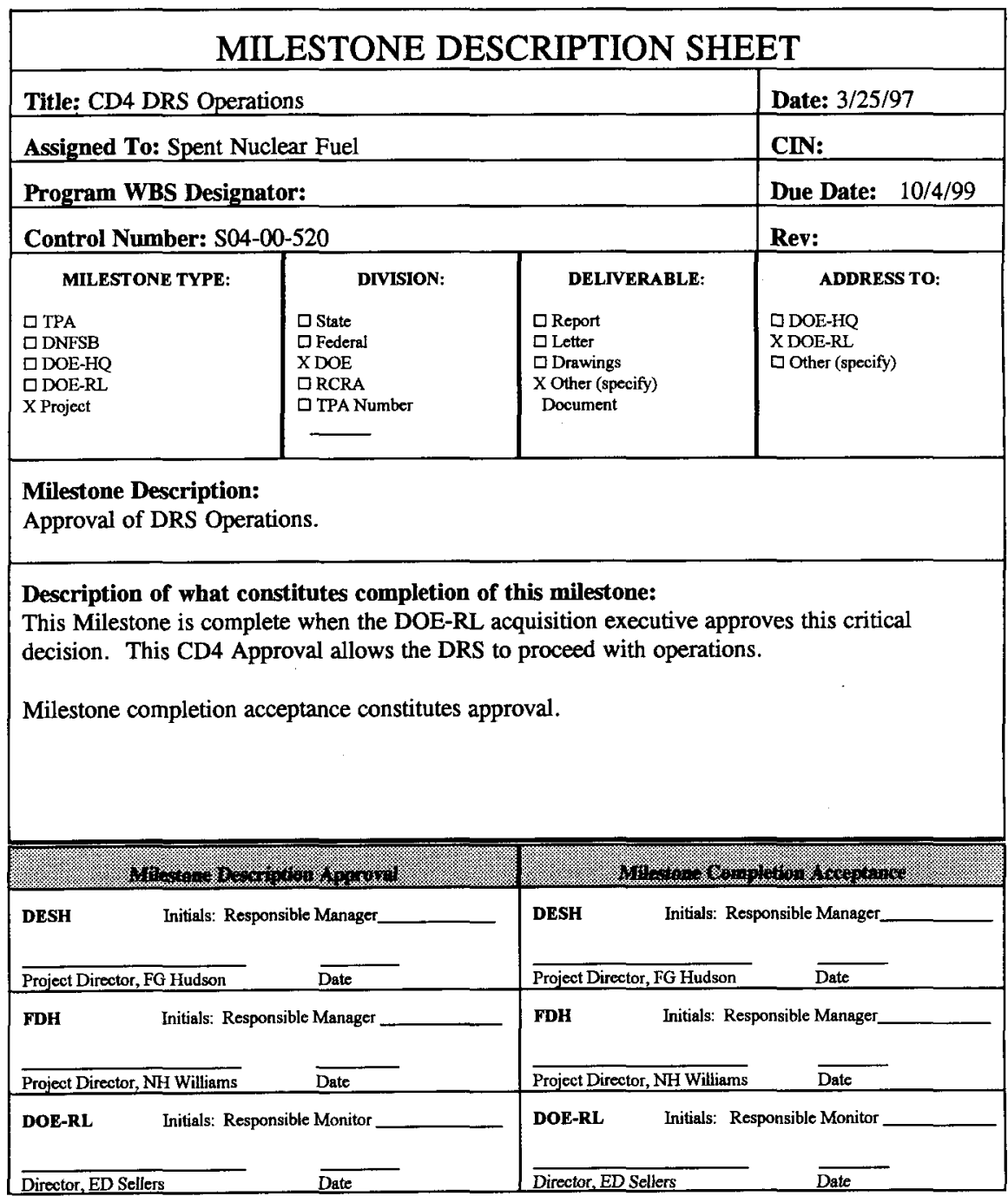




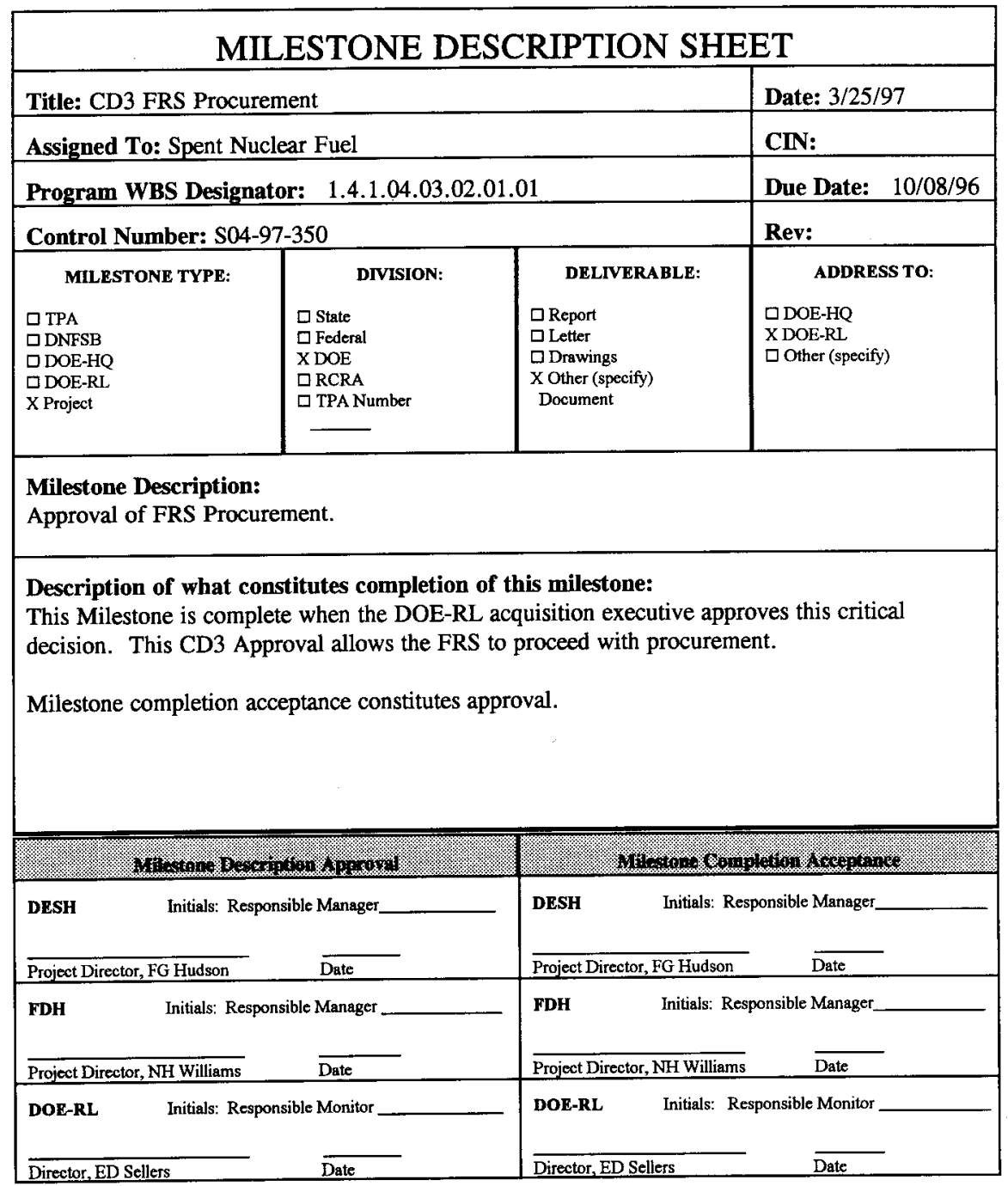




\section{SPENT NUCLEAR FUEL PROJECT \\ WBS 1.4.1}

HNF-SP-1 104, Rev 4

This page intentionally left blank. 
4.1.1 Budget Authority (BA) Summary by WBS

RL WBS 1.4.1

\begin{tabular}{ccrrrrrrrrrrrrr} 
PBS : & WDS TITLE & Prior Years & FY1997 & FY1998 & FY1999 & FY2000 & FY2001 & FY2002 & FY2003 & FY2004 & FY2005 & FY2006 & TOTAL \\
\hline RL-WM01 6696-0 & Project Direction & 1900 & 6854 & 13000 & 12000 & 6500 & 0 & 0 & 0 & 0 & 0 & 40254 \\
\hline Sub-Total Project Direction & & 1900 & 6854 & 13000 & 12000 & 6500 & 0 & 0 & 0 & 0 & 0 & 40254 \\
\hline
\end{tabular}

\begin{tabular}{|c|c|c|c|c|c|c|c|c|c|c|c|c|c|c|}
\hline RL-WM01 & $6690-0$ & QAES\&H & 471 & 785 & 258 & 263 & 270 & 50 & & & & & & 2097 \\
\hline RL-WM01 & $6690-0$ & Regulatory Compliance & 1836 & 1356 & 1332 & & & & & & & & & 4524 \\
\hline RL-WM01 & $6690-0$ & Characterization & 19000 & 6915 & 4638 & & & & & & & & & 30553 \\
\hline \multicolumn{3}{|c|}{ Sub-Total Safety \& Quality } & 21307 & 9056 & 6228 & 263 & 270 & 50 & 0 & 0 & 0 & 0 & 0 & 37174 \\
\hline
\end{tabular}

\begin{tabular}{llllllllllllll} 
RL-WM01 6696-0 Project Integration & 26665 & 13058 & 13871 & 13100 & 9618 & 6892 & 0 & 0 & 0 & 0 & 0 & $\mathbf{8 3 2 0 4}$ \\
\hline Sub-Total Project Integration & 26665 & 13058 & 13871 & 13100 & 9618 & 6892 & 0 & 0 & 0 & 0 & 0 & $\mathbf{8 3 2 0 4}$ \\
\hline
\end{tabular}

$\begin{array}{lllllllll}\text { RL-WM01 } 6696-0 & \text { Operate K Basins } & 29849 & 12045 & 12353 & 12689 & 13026 & 6800 & 86762\end{array}$

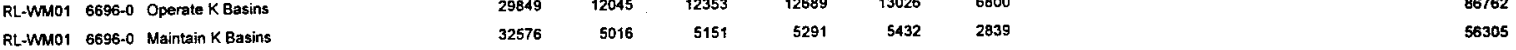

RL-WM01 6696-0 Provide/Maintain K Basin Baseline Doc $\quad 4482 \quad 2246 \quad 2307 \quad 2370 \quad 2433 \quad 1278 \quad 15116$

RL-WM01 6696-0 K Basin Staff Training

RL-WM01 6696-0 K Basin Management

$784 \quad 2532 \quad 2600$

$6003 \quad 3498 \quad 3593$

RL-WM01 6696-0 Fuet Relocation Common Operations

Sub-Total K Basin Operations \& Maintenance

$6638 \quad 1829$

15116

$\begin{array}{lll}2671 & 2742 & 0\end{array}$

987

\begin{tabular}{rrrr}
18294 & 12257 & 13473 & 0 \\
\hline 44298 & 38969 & 40895 & 12904
\end{tabular}

$73694 \quad 31975$

44298

50662

RL-WM01 6696-0 FRS K Basin Stantup/Training

$463 \quad 926 \quad 3705$

474

$\begin{array}{lllllll}0 & 0 & 0 & 0 & 0 & 242735\end{array}$

RL-WM01 6696-0 FRS K Basin Operations

$463 \quad 926 \quad 3705$

474

5568

RL-WM01 6696-0 Testing \& MSA of Transportation

Sub-Total FRS Operations

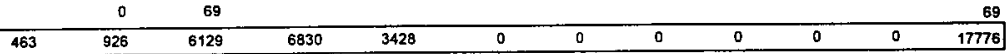

RL-WM01 6696-0 Debris Removal Operations

$760 \quad 1149-1145$

4

Sub-Total Debris Removal Operations

\begin{tabular}{llllllllllll}
760 & 1145 & 270 & 1896 & 2427 & 4 & & & & 6502 \\
\hline 760 & 1145 & 270 & 1896 & 2427 & 4 & 0 & 0 & 0 & 0 & 0 & 6502 \\
\hline
\end{tabular}

RL-WM01 6696-1 Cold Vacuum Drying Fac Startup \& MSA

RL-WMO1 6696-1 CVD Operations

Sub-Total CVD Operations

\begin{tabular}{|c|c|c|c|c|c|c|c|c|c|c|c|}
\hline \multirow[t]{2}{*}{228} & \multirow[t]{2}{*}{763} & 2283 & & & & & & & & & \multirow{2}{*}{$\begin{array}{r}3274 \\
11913\end{array}$} \\
\hline & & 1747 & 5471 & 4695 & & & & & & & \\
\hline 228 & 763 & 4030 & 5471 & 4695 & 0 & 0 & 0 & 0 & 0 & 0 & 15187 \\
\hline
\end{tabular}


4.1.1 Budget Authority (BA) Summary by WBS

RL WBS 1.4.1

\begin{tabular}{|c|c|c|c|c|c|c|c|c|c|c|c|c|c|c|}
\hline PBS \# & ADS \# & WES TITLE & Prior Years & FY1997 & FY1998 & FY1999 & FY2000 & FY2001 & FY2002 & FY2003 & FY2004 & FY2005 & FY2006 & TOTAL \\
\hline RL-WM01 & $6696-0$ & Water Treatment Operations & 1299 & 373 & 1646 & 1239 & 6351 & & & & & & & 10908 \\
\hline Sub-Total V & Nater Tre & atment Operations & 1299 & 373 & 1646 & 1239 & 6351 & 0 & 0 & 0 & 0 & 0 & 0 & 10908 \\
\hline
\end{tabular}

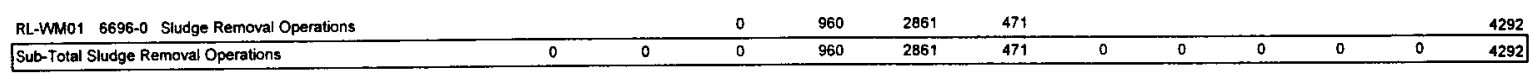

$\begin{array}{lllll}\text { RL-WMO1 6696-1 Hot Cond Sys Startup \& ORR } & 2192 & 403 & 2595\end{array}$

RL-WM01 6696-1 HCS Operations

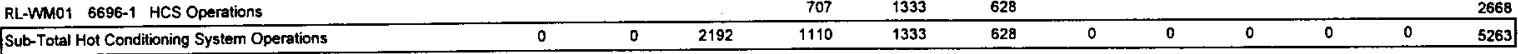

RL-WMD1 6696-1 CSB Startup \& MSA $\quad 600 \quad 912 \quad 2651 \quad 4163$

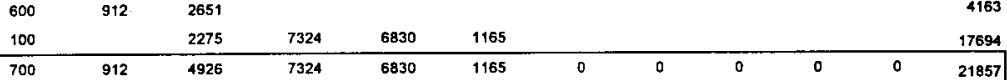

Sub-Total CSB Operations

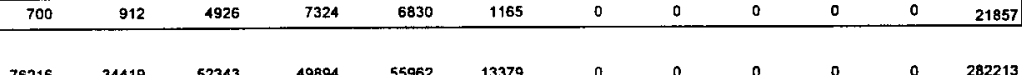

RL-WM01 6695-0 Sub-Total Operations

$\begin{array}{rrrrrrrrrrrr}76216 & 34419 & 52343 & 49894 & 55962 & 13379 & 0 & 0 & 0 & 0 & 0 & 282213 \\ 928 & 1675 & 11448 & 13905 & 12858 & 1793 & 0 & 0 & 0 & 0 & 0 & 42307\end{array}$

RL-WM01 6696-1 Sub-Total Operations

36094

63491

15172

$\begin{array}{rrrr}7439 & 2453 & 1413 & \\ 691 & 615 & 497 & 426\end{array}$

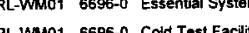

1100

RL-WM01 6696-0 Roof Repairs

RL-WM01 6696-0 Trailer Moves

RL.WM01 6696-0 Basin Personnel Facility Upgrades

RL-WM01 6696-0 Basin Facility Support Upgrades

RL-WM01 6696-0 Dose Reduction System

RL-WM01 6696-0 Basin Fac Upgrades for Transport

RL-WM01 6696-0 Deactivation Preparation

Sub-Total Facility Upgrades

123

$318 \quad 1498$

$258 \quad 34$

$5577 \quad 2041$

$782 \quad 4541 \quad 2250$

1551

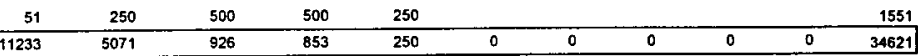


4.1.1 Budget Authority (BA) Summary by WBS

RL WBS 1.4.1

\begin{tabular}{|c|c|c|c|c|c|c|c|c|c|c|c|c|c|c|}
\hline PBS * & ADS * & WBS TITLE & Prior Years & FY1997 & FY1998 & FY1999 & FY2000 & FY2001 & FY2002 & FY2003 & FY2004 & FY2005 & FY2006 & TOTAL \\
\hline RL-WM01 & $6696-0$ & FRS Technical Baseline & 1793 & 308 & 20 & & & & & & & & & 2121 \\
\hline RL-WMO1 & $6696-0$ & FRS Project Management & 413 & 407 & 992 & 442 & & & & & & & & 2254 \\
\hline RL-WM01 & $6696-0$ & FRS Design & 3589 & 2431 & 527 & & & & & & & & & 6547 \\
\hline RL-WMo1 & $6696-0$ & FRS Proc/Fab/Construction & 226 & 6013 & 3830 & & & & & & & & & 10069 \\
\hline RL-WMO1 & $6696-0$ & FRS Equipment Test & 705 & 786 & & & & & & & & & & 1491 \\
\hline RL-WM01 & $6696-0$ & FRS Regulatory Compliance & 141 & 649 & 36 & & & & & & & & & 826 \\
\hline \multicolumn{3}{|c|}{ Sub-Total Fuet Removal } & 6867 & 10594 & 5405 & 442 & 0 & 0 & 0 & 0 & 0 & 0 & 0 & 23308 \\
\hline
\end{tabular}

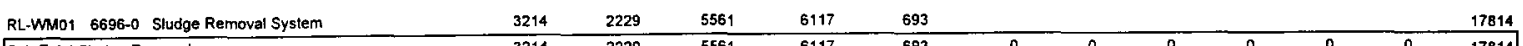

\begin{tabular}{|lllllllllllll}
\hline Sub-Total Sludge Removal & 3214 & 2229 & 5561 & 6117 & 693 & 0 & 0 & 0 & 0 & 0 & 0 & 17814 \\
\hline
\end{tabular}

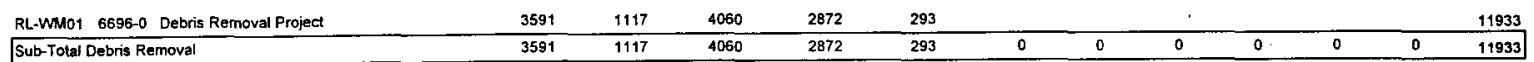

Sub-Total Debris Removal

\begin{tabular}{lllllll}
5862 & 8037 & 8624 & 1015 & 967 & 24505 \\
\hline
\end{tabular}

RL-WM01 6696-0 Water Treatment System

\begin{tabular}{llllllllll}
5862 & 8037 & 8624 & 1015 & 967 & 0 & 0 & 0 & 0 & 24505 \\
\hline
\end{tabular}

Total Facility Projects

3582

RL-WM01 6696-0 MCO ACquisition Definition

RL-WMO1 6696-0 MCO Project Management

RL-WM01 6596-0 MCO Design

RL-WMO1 6696-0 MCO Fabrication

RL-WMO1 6696-0 Testing \& Qualification of MCOs

RL-WM01 6696-0 MCO Topical Safety Report

\begin{tabular}{rr}
35822 & 3321 \\
\hline 1742 & \\
655 & 810 \\
1004 & 9 \\
& 174 \\
925 & 147 \\
190 & 256 \\
4516 & 39 \\
\hline
\end{tabular}

33210

$28721 \quad 11372 \quad 2806$

250

$0 \quad 0$

0

0

0

112181

Sub-Total Multi Canister Overpack (MCO)

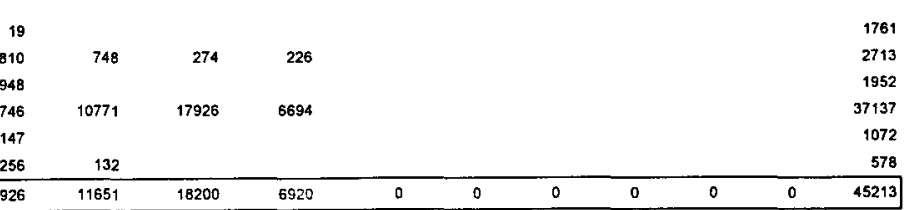




\section{SPENT NUCLEAR FUEL PROJECT WBS 1.4.1}

HNF-SP-1 104, Rev 4

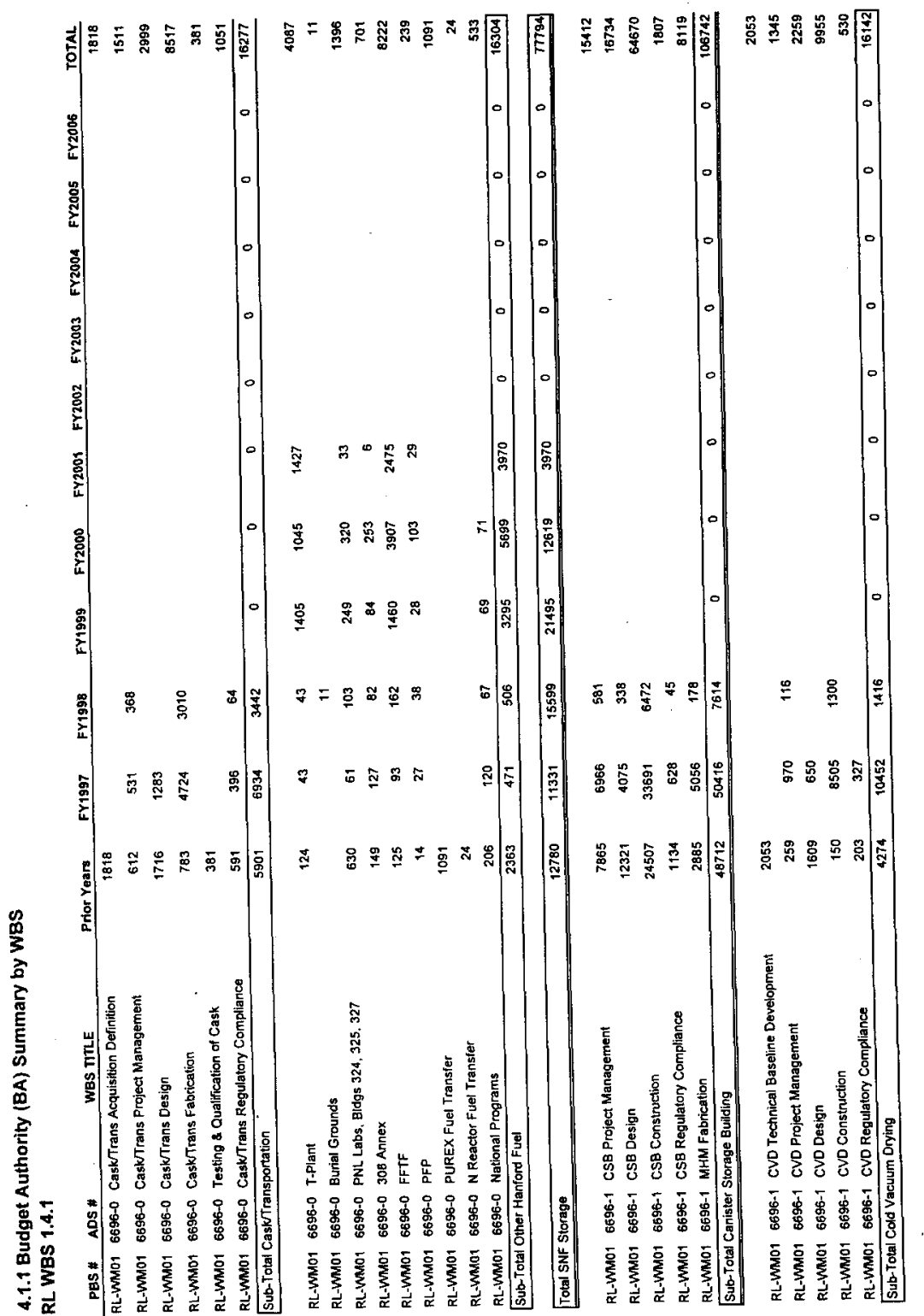




\subsubsection{Budget Authority (BA) Summary by WBS}

RL WBS 1.4.1

PBS \# ADS \# WBS TITLE Prior Years

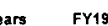

\begin{tabular}{|c|c|c|c|c|c|c|c|c|c|c|c|}
\hline lears & FY1997 & FY1998 & FY1999 & FY2000 & FY2001 & FY2002 & FY2003 & FY2004 & FY2005 & FY2006 & TOTAL \\
\hline 966 & 201 & & & & & & & & & & $\$ 167$ \\
\hline 167 & 1720 & 1288 & & & & & & & & & 3175 \\
\hline 1462 & 2833 & & & & & & & & & & 4295 \\
\hline 25 & 123 & 6502 & & & & & & & & & 6650 \\
\hline 300 & 493 & 226 & & & & & & & & & 1019 \\
\hline 2920 & 5370 & 8016 & 0 & 0 & 0 & 0 & 0 & 0 & 0 & 0 & 16306 \\
\hline
\end{tabular}

Sub-Total Hot Conditioning System

$7194 \quad 15822$

9432

0

0

0

RL-WM01 6696-0 Total SNF Projects

17279

RL-WM01 6696-1 Total SNF Projects

$172790 \quad 102974$

$123616 \quad 109124$

31041

56834

RL-WMO1 GRANO TOTAL SNF PROJECTS

229624

$102974 \quad 123616$

13905

12858

1793

$\begin{array}{llllll}0 & 0 & 0 & 0 & 0 & 632820 \\ 0 & 0 & 0 & 0 & 0 & 181497\end{array}$

\section{HQ/RL Assessments}

170887

151810

123029

106133

32834

0

$229624 \quad 175000$

151810

123029

106133

32834

$\begin{array}{lllll}0 & 0 & 0 & 0 & 818430\end{array}$

in

( 


\section{SPENT NUCLEAR FUEL PROJECT \\ WBS 1.4.1}

HNF-SP-1104, Rev 4

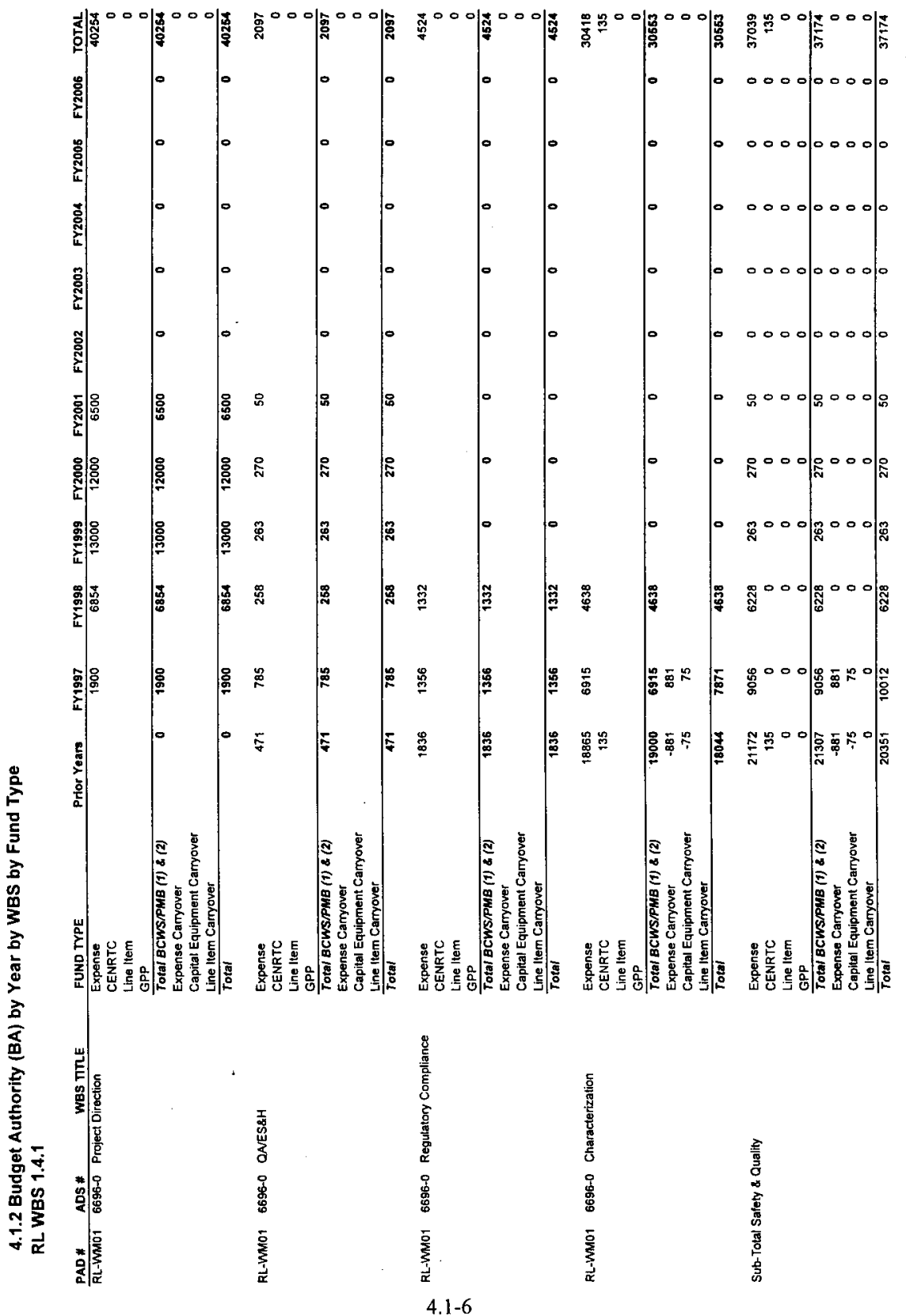




\section{SPENT NUCLEAR FUEL PROJECT \\ WBS 1.4.1}

HNF-SP-1 104, Rev 4

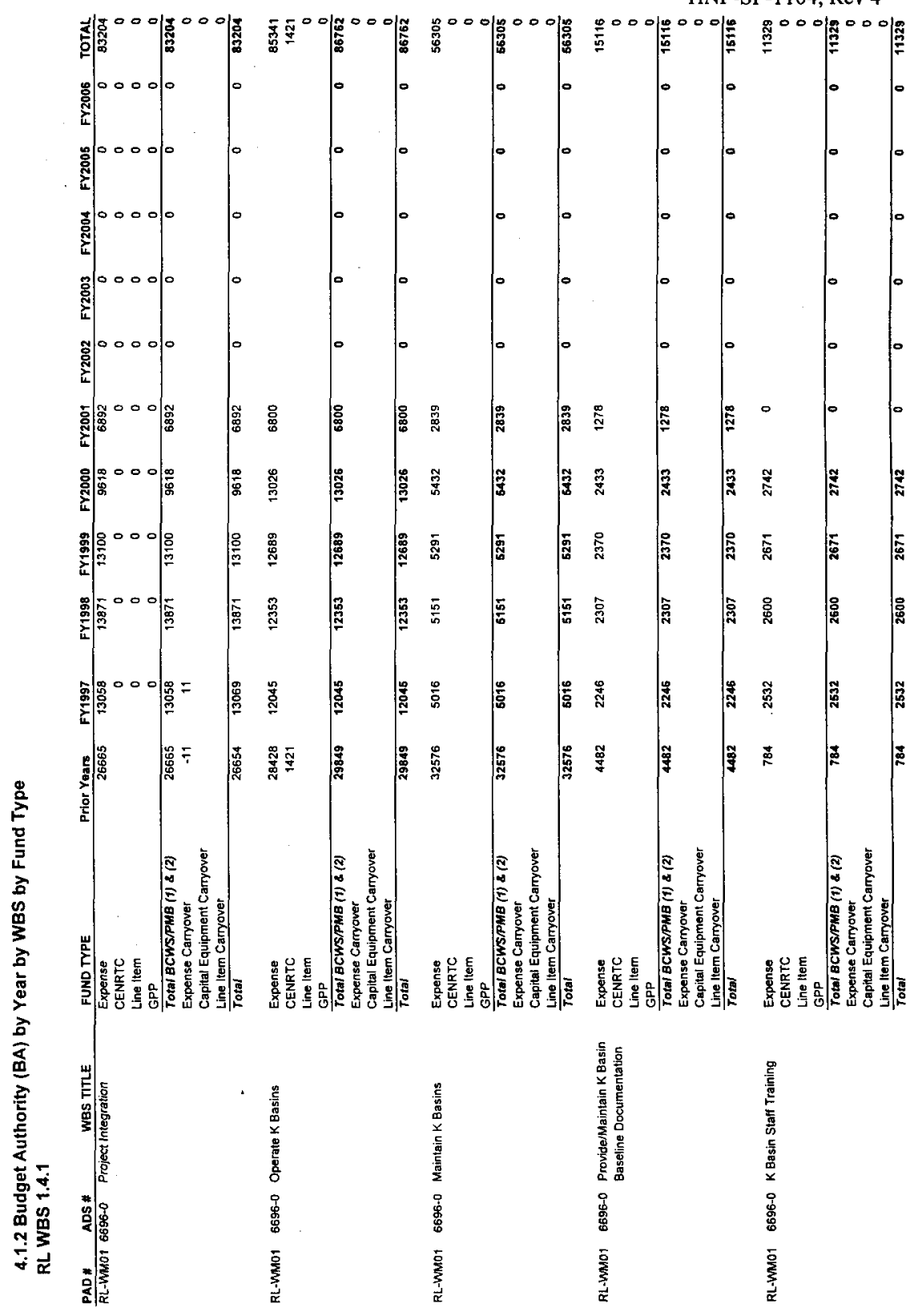


4.1.2 Budget Authority (BA) by Year by WBS by Fund Type

RL WBS 1.4.1

PAD* ADS * WBS TITLE

RL-WMO1 6696-0 KBasin Management

FUND TYPE

Expense

Prior Years

CENRTC

Line Item

\begin{tabular}{ll} 
GPP & \\
Line Item & \\
\hline
\end{tabular}

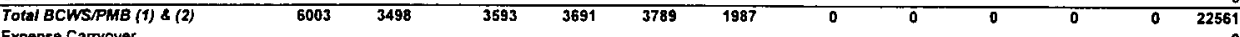

Expense Carryover

Capital Equipment Carryover

Line Item Carryover

Total

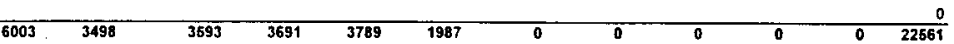

RL-WMO1 6696-0 Fuel Relocation Common Expense

Operations CENRTC

$\begin{array}{lllll}6638 & 18294 & 12257 & 13473 & 0\end{array}$

Line Item

Line Item

GPP

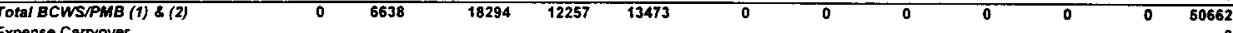

Expense Carnover

Capital Equipment Carryover

Line Item Carryover

Total

Expense

EXpense

Line Item

Total BCWS/PMB (1) \& (2)

Expense Carryover

Caprial Equipment Carryover

Line Item Canyover

Totel

$\begin{array}{rr}0 & 66 \\ 22273 & 31975 \\ 1421 & \\ 0 & \\ 0 & \\ 73694 & 31975 \\ 0 & \\ 0 & \\ 0 & \\ 73694 & 31975\end{array}$

RL-WMO1 6696-0 FRS K Basin Startup' Training

Expense

CENRTC

Line Item

GPP

Tota BCWS/PMB (1) \& (2)

$\begin{array}{llll}463 & 926 & 3705 & 474\end{array}$

Expense Carryover

$463-926$

$3705-474$

\begin{tabular}{lll} 
& & \\
\hline & 0 & 0
\end{tabular}

Capital Equipment Carryover

Line Item Carryover

Total

$463 \quad 926$

0 I

RL-WM01 6696-0 FRS K Basin Operations

Expense

CENRTC

Line Iter

GPP

TOES BCWS/PME (1) \& (2)

Expense Carryover

Capital Equipment Carryover

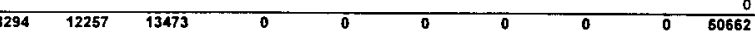

Laphe Equipment Cam

Total

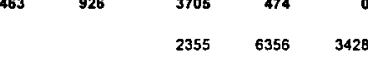

12904
0
0
0
12904
0
0
0
12904

2355

o 
4.1.2 Budget Authority (BA) by Year by WBS by Fund Type

RL WBS 1.4.1

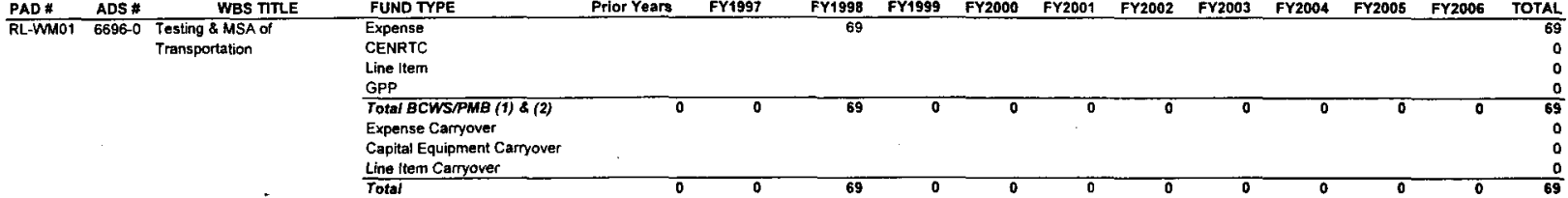

Sub-Total FRS Operations

$$
\text { rotaj }
$$

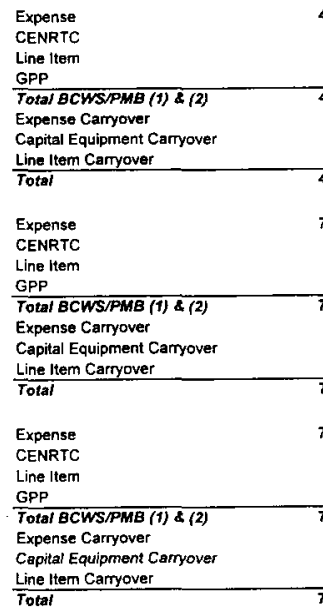

RL-WMO1 6696-1 Cold Vacuum Drying Fac. Startup \& MSA

Debris Removal

Operations

Sub-Total Debris Removal Operations

\section{Expense \\ CENRTC}

Line ltem

GPP

Total BCWS/PME (1) \& (2)

Expense Carryover

Capital Equipment Carryover

Line Item Carryover

Total

$\begin{array}{rrr}463 & 926 & \\ 0 & 0 & \\ 0 & 0 & \\ 0 & 0 & \\ 463 & 926 & 6 \\ 0 & 0 & \\ 0 & 0 & \\ 0 & 0 & \\ 463 & 926 & 6\end{array}$

$760-1145$

$1896-2427$

4

$760 \quad 1145$

$\begin{array}{rrr}760 & 1145 & 27 \\ 0 & 0 & \\ 0 & 0 & \\ 0 & 0 & \\ 760 & 1145 & 27 \\ 0 & 0 & \\ 0 & 0 & \\ 0 & 0 & \\ 760 & 1145 & 270\end{array}$

\begin{tabular}{rr}
270 & 1896 \\
270 & 189 \\
0 & \\
0 & \\
0 & \\
270 & 189 \\
0 & \\
0 & \\
0 & \\
270 & \\
\hline
\end{tabular}

$\begin{array}{r}1896 \\ 0 \\ 0 \\ 0 \\ \hline 1896 \\ 0 \\ 0 \\ 0 \\ \hline 1896\end{array}$

2427

2427

\begin{tabular}{rl}
427 & 4 \\
0 & 0 \\
0 & 0 \\
0 & 0 \\
\hline 427 & 4 \\
0 & 0 \\
0 & 0 \\
0 & 0 \\
\hline 427 & 4
\end{tabular}

\begin{tabular}{l}
0 \\
0 \\
0 \\
0 \\
0 \\
0 \\
0 \\
0 \\
\hline 0
\end{tabular}

\begin{tabular}{l}
0 \\
0 \\
0 \\
0 \\
0 \\
0 \\
0 \\
0 \\
\hline
\end{tabular}

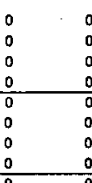

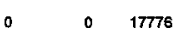

$\begin{array}{ll}0 & 0 \\ 0 & 0\end{array}$

$\begin{array}{lll}0 & 0 & 0 \\ 0 & 0 & 17776\end{array}$

$\begin{array}{r}0 \\ 0 \quad 0 \\ \hline\end{array}$

7776

\begin{tabular}{l}
0 \\
0 \\
\hline
\end{tabular}

502

0

0

$\begin{array}{lll}228 & 763 & 2283\end{array}$

$228 \quad 763$

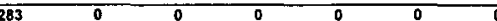

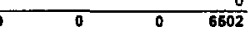

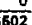

0 
4.1.2 Budget Authority (BA) by Year by WBS by Fund Type

RL. WBS 1.4.1

\begin{tabular}{|c|c|c|c|c|c|c|c|c|c|c|c|c|c|c|c|}
\hline \multirow{10}{*}{$\frac{\text { PAD \# }}{\text { RL-WM01 }}$} & \multirow{2}{*}{$\frac{A D S \#}{6696-1}$} & \multirow{2}{*}{$\frac{\text { Wes TITLE }}{\text { CVD Operations }}$} & FUND TYPE & \multirow[t]{2}{*}{ Prior Years } & FY4997 & \multirow{2}{*}{$\begin{array}{r}\text { FY1998 } \\
1747\end{array}$} & \multirow{2}{*}{$\frac{\text { FY1999 }}{5471}$} & \multirow{2}{*}{$\frac{F Y 2000}{4695}$} & FY2001 & FY2002 & FY2003 & FY2004 & FY2005 & FY2006 & TOTAL \\
\hline & & & Expense & & & & & & & & & & & & 11913 \\
\hline & & & CENRTC & & & & & & & & & & & & 0 \\
\hline & & & Line liem & & & & & & & & & & & & 0 \\
\hline & & & GPP & & & & & & & & & & & & 0 \\
\hline & & & Total BCWS/PMB (1) \& (2) & 0 & 0 & 1747 & 5471 & 4695 & $\overline{0}$ & 0 & $\mathbf{0}$ & 0 & 0 & 0 & 11913 \\
\hline & & & Expense Carryover & & & & & & & & & & & & 0 \\
\hline & & & Capital Equipment Carryover & & & & & & & & & & & & 0 \\
\hline & & & Line Item Carryover & & & & & & & & & & & & 0 \\
\hline & & . & Total & $\bar{c}$ & 0 & $\overline{1747}$ & 5471 & 4695 & 0 & 0 & $\overline{0}$ & $\overline{0}$ & 0 & 0 & $\overline{11913}$ \\
\hline
\end{tabular}

Sub-Total CVD Operations

\begin{tabular}{l} 
Expense \\
CENRTC \\
Line ltem \\
GPP \\
\hline Total BCWSIPMB (1) \& (2) \\
Expense Caryover \\
Capital Equipment Carryover \\
Line ltem Carmover \\
Total
\end{tabular}

D RL-WM01 6696-0 Water Treatment
Operations
D

Total

\begin{tabular}{rrrrrl}
228 & 763 & $\mathbf{4 0 3 0}$ & $\mathbf{5 4 7 1}$ & $\mathbf{4 6 9 5}$ & 0 \\
0 & 0 & 0 & 0 & 0 & 0 \\
0 & 0 & 0 & 0 & 0 & 0 \\
0 & 0 & 0 & 0 & 0 & 0 \\
\hline 228 & 763 & $\mathbf{4 0 3 0}$ & $\mathbf{5 4 7 1}$ & $\mathbf{4 6 9 5}$ & 0 \\
0 & 0 & 0 & 0 & 0 & 0 \\
0 & 0 & 0 & 0 & 0 & 0 \\
0 & 0 & 0 & 0 & 0 & 0 \\
\hline 228 & 763 & 4030 & 5471 & $\mathbf{4 6 9 5}$ & 0
\end{tabular}

\begin{tabular}{l}
0 \\
0 \\
0 \\
0 \\
0 \\
0 \\
0 \\
0 \\
\hline 0
\end{tabular}

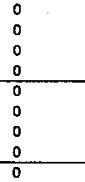

Expense

$\begin{array}{lllll}1299 & 373 & 1546 & 1239 & 6351\end{array}$

CENRTC

Line Item

Total BCWS/PMB (1) \& (2)

$\begin{array}{llll}0 & 0 & 0 & 15187\end{array}$

Capital Equipment Carryover

Line liem Carryover

299

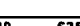

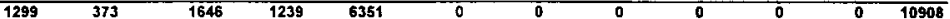

Sub-Total Water Treatment Operations

Expense

CENRTC

Line ltem

GPP

Total BCWS/PME (1) \& (2)

Expense Carryover

Capital Equipment Carryover

Line ltem Carryover

Total

Expense

CENRTC

Line liem

Line

\begin{tabular}{rrr}
299 & 373 & 164 \\
0 & 0 & \\
0 & 0 & \\
0 & 0 & \\
299 & $\mathbf{3 7 3}$ & 1646 \\
0 & 0 & \\
0 & 0 & \\
0 & 0 & \\
\hline 299 & 373 & 1646
\end{tabular}

RL-WM01 6696-D Sludge Removal Operations

TotaI BCWS/PMB (1) \& (2)

Expense Camyover

Capital Equipment Carryover

Line liem Carryover

$\begin{array}{lllll}0 & 960 & 2861 & 471\end{array}$

\begin{tabular}{rrr}
239 & 6351 & 0 \\
0 & 0 & 0 \\
0 & 0 & 0 \\
0 & 0 & 0 \\
\hline 39 & $\mathbf{6 3 5 1}$ & 0 \\
0 & 0 & 0 \\
0 & 0 & 0 \\
0 & 0 & 0 \\
\hline 239 & $\mathbf{6 3 5 1}$ & 0
\end{tabular}

\begin{tabular}{l}
0 \\
0 \\
0 \\
0 \\
0 \\
0 \\
0 \\
0 \\
\hline 0
\end{tabular}

\begin{tabular}{l}
0 \\
0 \\
0 \\
0 \\
0 \\
0 \\
0 \\
0 \\
\hline 0
\end{tabular}

$\begin{array}{llll}0 & 0 & 0 & 10908\end{array}$

Line Item Carryover

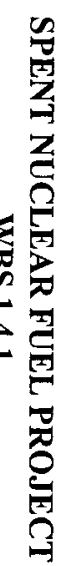


4.1.2 Budget Authority (BA) by Year by WBS by Fund Type RL WBS 1.4.1

PAD * ADS * WES TITLE Sub-Total SIudge Removal Operations

$=$

RL-WM01 6696-1 Hot Cond Sys Startup and $O R R$

\begin{tabular}{|c|c|}
\hline FUND TYPE & Prior Years \\
\hline Expense & $\overline{0}$ \\
\hline CENRTC & 0 \\
\hline Line ftem & 0 \\
\hline GPP & 0 \\
\hline Total BCWS/PMB (1) \& (2) & 0 \\
\hline Expense Carryover & 0 \\
\hline Capital Equipment Carryover & 0 \\
\hline Line Item Carryover & 0 \\
\hline Total & 0 \\
\hline
\end{tabular}

Sub-Total HCS Operations

RL-WM01 6696-1 CSB Startup and MSA

Expense

CENRTC

Line Item

GPP

Total BCWS/PMB (1) \& (2)

Expense Carryover

Capital Equipment Carryover

Line ltem Carryover

Total

Expense

CENRTC

Line liem

GPP

Total BCWS/PMB (1) \& (2)

Expense Carryover

Capital Equipment Carryover

Line Item Carryover

Total

Expense

CENRTC

Line lit

TPP

Expense Cartyover

Capital Equipment Carryover

Line Item Carryover

Total

Expense

CENRTC
Tolal BCWS/PMB (1)\& (2)

$\begin{array}{rrr}0 & \text { FY1998 } & \text { FY19g } \\ 0 & 0 & \\ 0 & 0 \\ 0 & 0 \\ 0 & 0 & 0 \\ 0 & 0 \\ 0 & 0 \\ 0 & 0 \\ 0 & 0\end{array}$
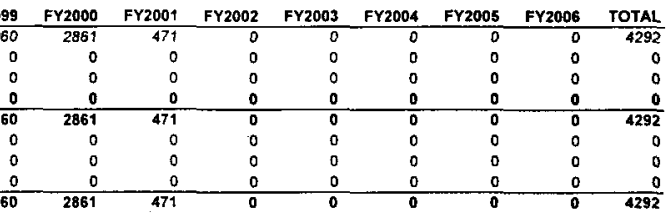

Line Item

$2192 \quad 403$

103

2595

03
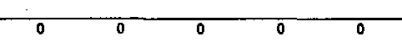

403

0

0

$\begin{array}{lll}707 & 1333 \quad 628\end{array}$

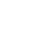

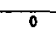

0

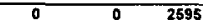

Total BCWSAPMB (1) \& (2)

Expense Carryover

Capital Equipment Carryover

Line Item Carryover

$$
\text { Total }
$$

0

-

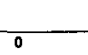

\begin{tabular}{lll}
0 & 0 & 2 \\
0 & 0 & \\
0 & 0 & \\
0 & 0 & 0 \\
0 & 0 & 2192 \\
0 & 0 & \\
0 & 0 & \\
0 & 0 & 0 \\
\hline 0 & 0 & 2192
\end{tabular}

\begin{tabular}{rr}
0 & 707 \\
2192 & 1110 \\
0 & 0 \\
0 & 0 \\
0 & 0 \\
\hline 2192 & 1110 \\
0 & 0 \\
0 & 0 \\
0 & 0 \\
\hline 2192 & 1110
\end{tabular}

\begin{tabular}{|c|}
\hline 133 \\
\hline 33 \\
\hline 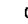 \\
\hline 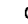 \\
\hline 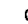 \\
\hline 133 \\
\hline 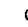 \\
\hline 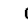 \\
\hline 0 \\
\hline
\end{tabular}

$\begin{array}{lll}0 & 0 & 0 \\ 0 & 0 & 0 \\ 0 & 0 & 0 \\ 0 & 0 & 0 \\ 0 & 0 & 0 \\ 0 & 0 & 0 \\ 0 & 0 & 0 \\ 0 & 0 & 0 \\ 0 & 0 & 0\end{array}$

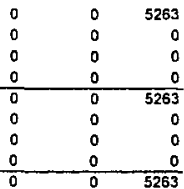

0 盯

4163

$600 \quad 912 \quad 2651$

0

0

163

0 2

$0 \rightarrow$

628
628
0
0
0
628
0
0
0
628

\begin{tabular}{ll}
0 & 0 \\
0 & 0 \\
0 & 0 \\
0 & 0 \\
\hline 0 & 0 \\
0 & 0 \\
0 & 0 \\
0 & 0 \\
\hline 0 & 0
\end{tabular}

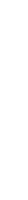

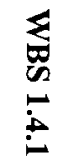


4.1.2 Budget Authority (BA) by Year by WBS by Fund Type

RL WBS 1.4.1

\begin{tabular}{|c|c|c|c|c|c|c|c|c|c|c|c|c|c|c|c|}
\hline PAD \# & ADS \# & WBS TITLE & FUND TYPE & Prior Years & FY1997 & FY1998 & FY1999 & FY2000 & FY2001 & FY2002 & FY2003 & FY2004 & FY2005 & FY2006 & TOTAL \\
\hline \multirow[t]{9}{*}{ RL-WMO1 } & $6696-1$ & Operations of CSB & Expense & 100 & & 2275 & 7324 & 6830 & 1165 & & & & & & 17694 \\
\hline & & & CENRTC & & & & & & & & & & & & 0 \\
\hline & & & Line Item & & & & & & & & & & & & 0 \\
\hline & & & GPP & & & & & & & & & & & & 0 \\
\hline & & & Total BCWS/PMB (1) \& (2) & 100 & 0 & 2275 & 7324 & 6830 & 1165 & 0 & $\overline{0}$ & 0 & 0 & $\overline{0}$ & 17694 \\
\hline & & & Expense Carryover & & & & & & & & & & & & 0 \\
\hline & & & Capital Equipment Carryover & & & & & & & & & & & & 0 \\
\hline & & & Line Item Carryover & & & & & & & & & & & & 0 \\
\hline & & & Total & 100 & $\mathbf{0}$ & 2275 & 7324 & 6830 & 1165 & 0 & 0 & 0 & o & o & 4 \\
\hline
\end{tabular}

Sub-Total CSB Operations

\begin{tabular}{|c|c|c|c|c|c|}
\hline Expense & 700 & 912 & 4926 & 7324 & 6830 \\
\hline CENRTC & 0 & 0 & 0 & 0 & 0 \\
\hline Line item & 0 & 0 & 0 & 0 & 0 \\
\hline GPP & 0 & 0 & 0 & 0 & 0 \\
\hline rotal BCWS/PMB (1) \& (2) & $\overline{700}$ & 912 & 4926 & 7324 & 6830 \\
\hline Expense Carryover & 0 & 0 & 0 & 0 & 0 \\
\hline Capital Equipment Carryover & 0 & 0 & 0 & 0 & 0 \\
\hline Line ltem Carryover & 0 & 0 & 0 & 0 & 0 \\
\hline Total & 700 & 912 & 4926 & 7324 & 6830 \\
\hline Expense & 74795 & 34419 & 52343 & 49894 & 55962 \\
\hline CENRTC & 1421 & 0 & 0 & 0 & 0 \\
\hline Line Item & 0 & 0 & 0 & 0 & 0 \\
\hline GPP & 0 & 0 & 0 & 0 & 0 \\
\hline Total BCWSTPM (1) \& (2) & 76216 & 34419 & 52343 & 49894 & 55962 \\
\hline Expense Carryover & 0 & 0 & 0 & 0 & 0 \\
\hline Capital Equipment Carryover & 0 & 0 & 0 & 0 & 0 \\
\hline Line Item Carryover & 0 & 0 & 0 & 0 & 0 \\
\hline
\end{tabular}

RL-WM01 6696-0 Sub-Total Operations

$76216 \quad 34419$

52343

0

$\begin{array}{rl}165 & 0 \\ 0 & 0 \\ 0 & 0 \\ 0 & 0 \\ 165 & 0 \\ 0 & 0 \\ 0 & 0 \\ 0 & 0 \\ 1165 & 0\end{array}$

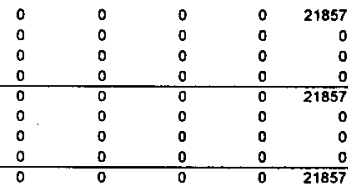

RL-WM01 6696-1 Sub-Total Operations

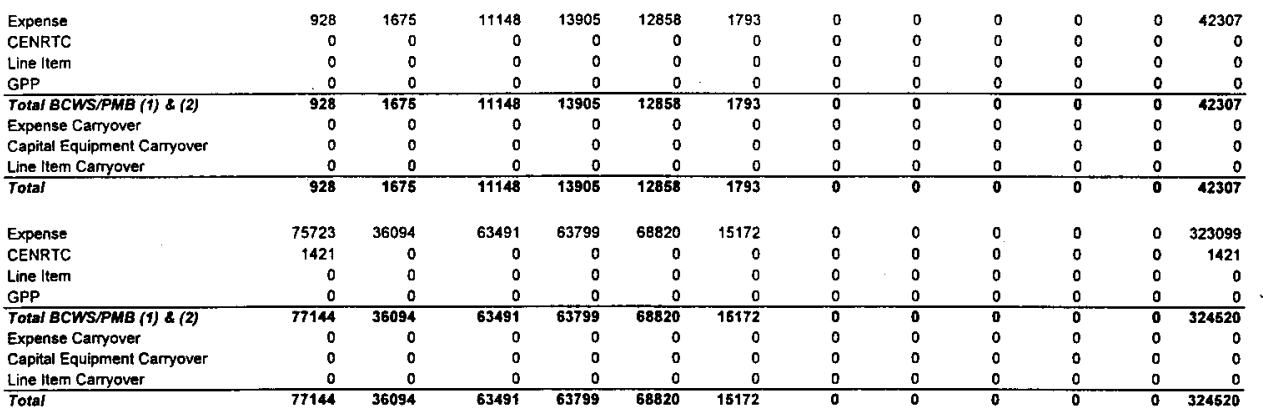


4.1.2 Budget Authority (BA) by Year by WBS by Fund Type

RL WBS 1.4.1

PAD \# ADS \# WBS TITLE

FUND TYPE

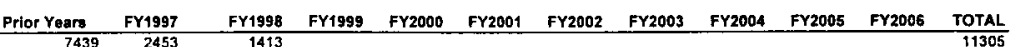

Expense

Line lte

GPP

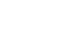

Toraibchsmor

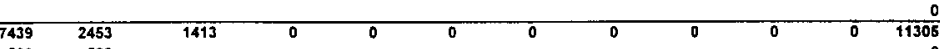

Capital Equipment Carryover

500

Line ltem Carryover

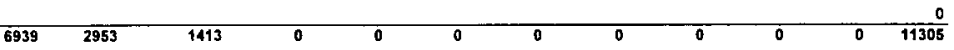

RL-WM01 6696-0 Cold Test Facility

Expense

$\begin{array}{lllll}691 & 615 & 497 & 426 & 353\end{array}$

Line liem

Line

0
0

Total BCWS/PMB (1) \& (2)

Expense Carryover

Capital Equipment Carryover

Line Item Carryover

Total

Expense

CENRTC

Line ltem

GPP

Total BCWS/PME (1) \& (2)

Expense Cartyover

Capital Equipment Carryover

Line item Carryover

Total

Expense

CENRTR

Line Item

Total BCWS/PMB (1) \& (2)

$615 \quad 497$

Capital Equipment Carryover

Line Item Cartyover

Tota!

$\begin{array}{llllllllll} & & & & & & & 0 \\ \end{array}$

Expense

CENRTC

Line It

GPP

Total BCWS/PMB

Capital Equipment Carryover

Line Item Carryover

Total

$\begin{array}{lllllllll} & & & & & & & & 0 \\ 0\end{array}$

(2)

$\begin{array}{lllllllll}0 & 0 & 0 & 0 & 0 & 0 & 0 & 0 & 0\end{array}$

123

0

o

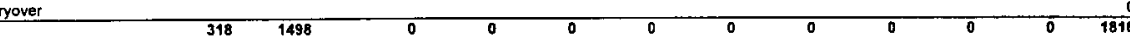


WBS 1.4.1

HNF-SP-1104, Rev 4

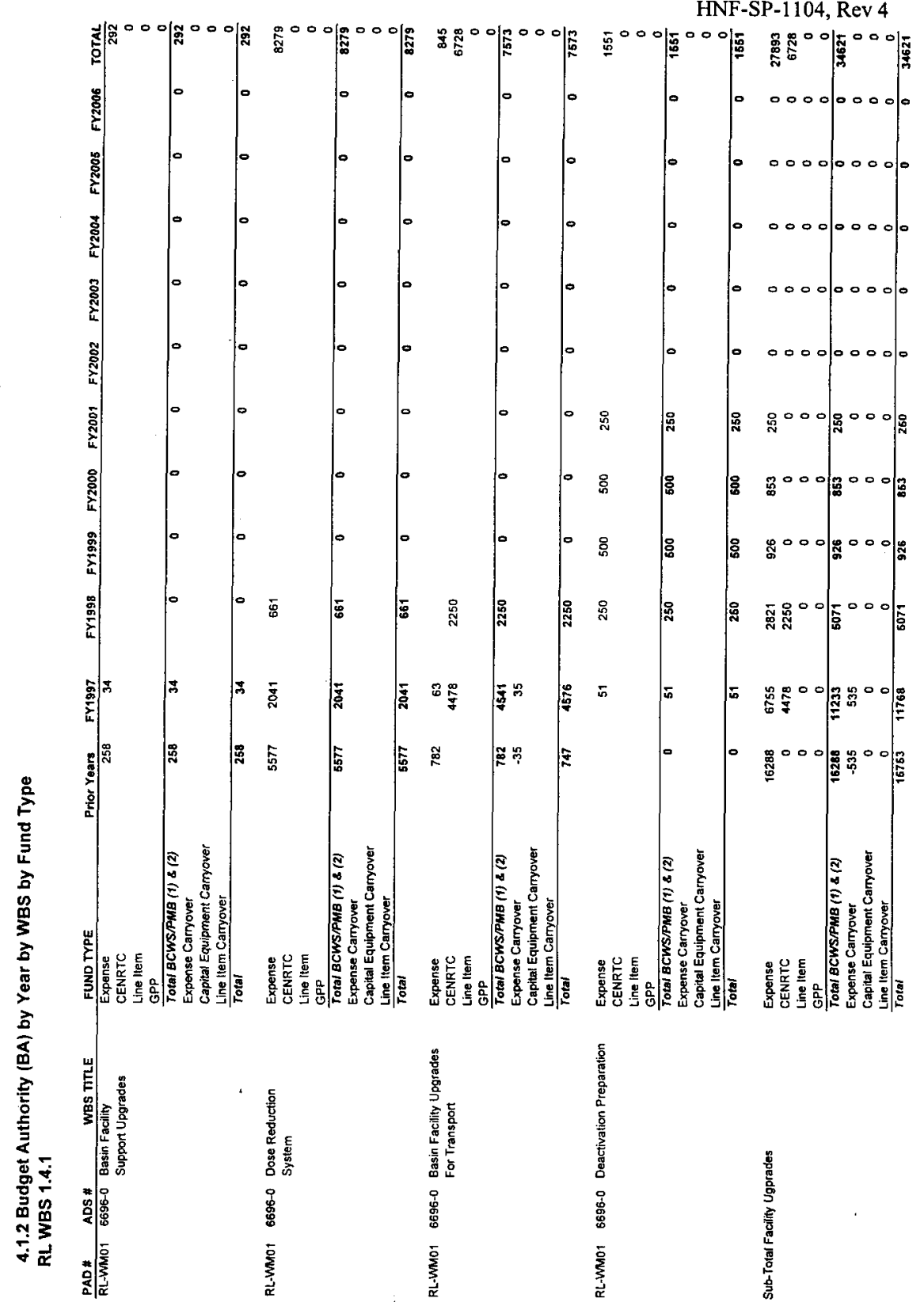




\section{SPENT NUCLEAR FUEL PROJECT WBS 1.4.1}

HNF-SP-1 104, Rev 4

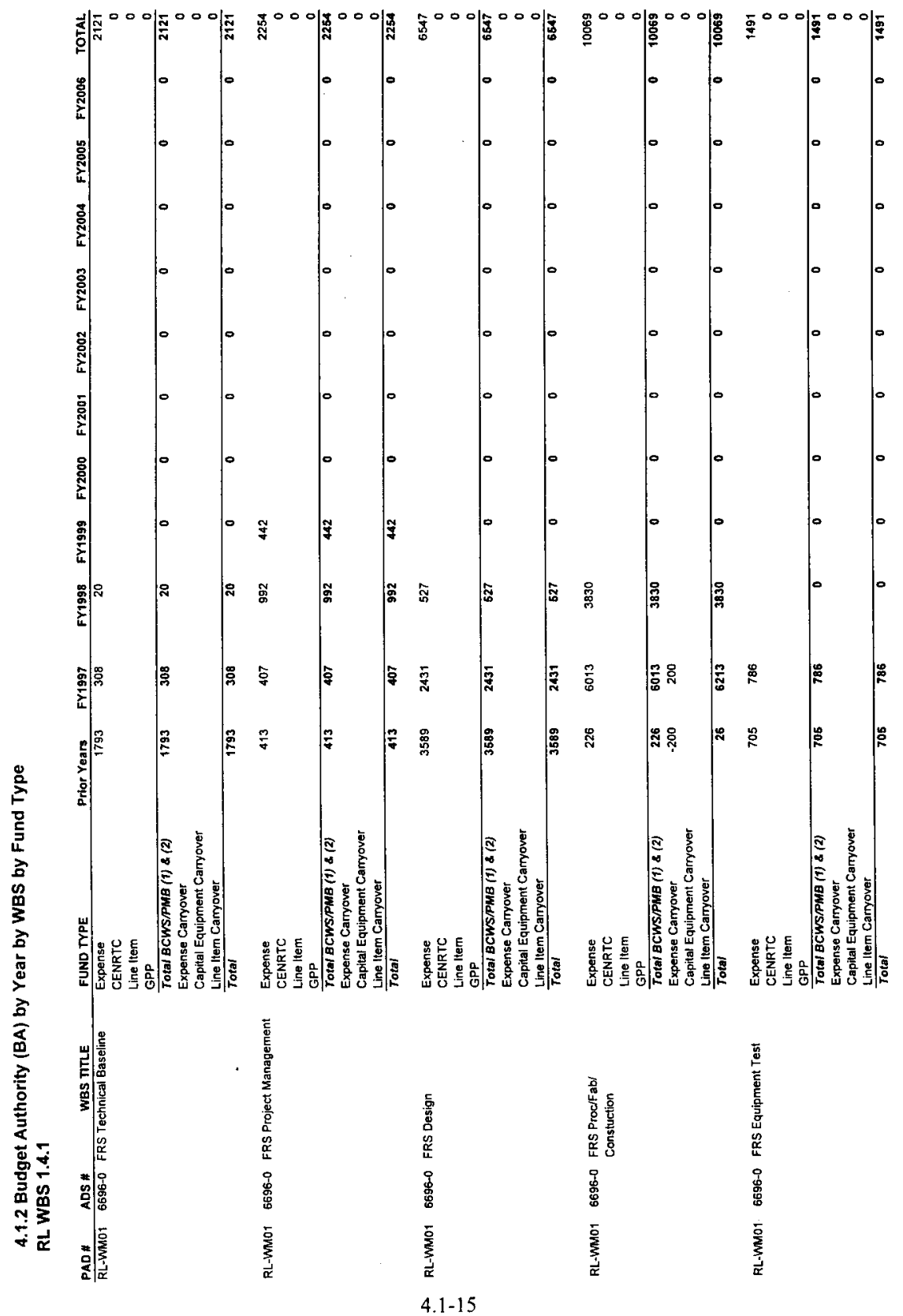


4.1.2 Budget Authority (BA) by Year by WBS by Fund Type RL WBS 1.4.1

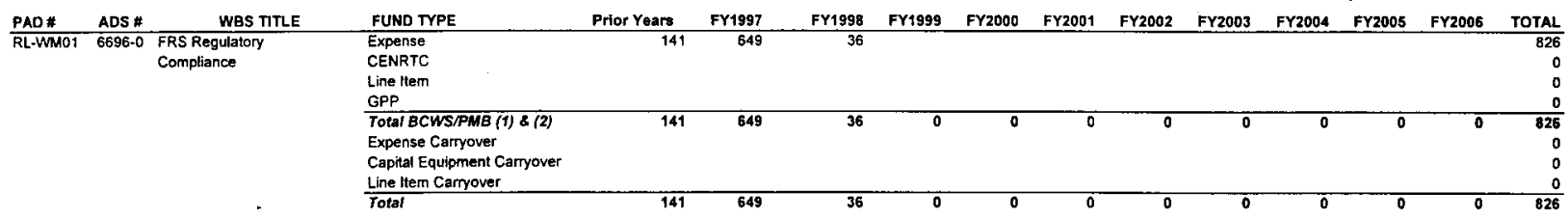

Sub-Total Fuel Removal

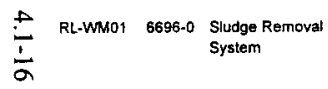

Expense
CENRTC
Line Item
GPP
Total BCWSPMB (1) \& (2)
Expense Carryover
Capital Equipment Carryover
Line ltem Carryover
Total

\begin{tabular}{rrr}
6867 & 10594 & 5 \\
0 & 0 & \\
0 & 0 & \\
0 & 0 & \\
\hline 6867 & 10594 & 5405 \\
-200 & 200 & \\
0 & 0 & \\
0 & 0 & \\
\hline 6667 & 10794 & 5
\end{tabular}

\begin{tabular}{rr}
405 & 4 \\
0 & \\
0 & \\
0 & \\
405 & \\
0 & \\
0 & \\
0 & \\
\hline 405 & 4 \\
\hline
\end{tabular}

\begin{tabular}{rl}
442 & 0 \\
0 & 0 \\
0 & 0 \\
0 & 0 \\
\hline 42 & 0 \\
0 & 0 \\
0 & 0 \\
0 & 0 \\
\hline 442 & 0
\end{tabular}

$\begin{array}{ll}0 & 0 \\ 0 & \\ 0 & 0 \\ 0 & 0 \\ 0 \\ 0 \\ 0 \\ 0 \\ 0\end{array}$

\begin{tabular}{lll}
0 & 0 & 0 \\
0 & 0 & 0 \\
0 & 0 & 0 \\
0 & 0 & 0 \\
0 & 0 & 0 \\
0 & 0 & 0 \\
0 & 0 & 0 \\
0 & 0 & 0 \\
\hline 0 & 0 & 0
\end{tabular}

$\begin{array}{ll}0 & 0 \\ 0 & 0 \\ 0 & 0 \\ 0 & 0 \\ 0 & 0 \\ 0 & 0 \\ 0 & 0 \\ 0\end{array}$

$0 \quad 0 \quad 23308$

Expense

$\begin{array}{lllll}3214 & 2229 & 5561 & 6117 & 693\end{array}$

CENRTC

Line Item

GPP

Total BCWS/PMB (1) \& (2)

3

Expense Carryover

Capital Equipment Carryover

Line Item Carryover

Total

$\begin{array}{lllll}3214 & 2229 & 5561 & 6117 & 693\end{array}$

93

$\begin{array}{rrrrr}0 & 0 & 0 & 0 & 17814\end{array}$

Sub-Total Sludge Removal

Expense

CENRTC

Line ttem

GPP

Total BCWS/PMB (1) \& (2)

Expense Carryover

Capital Equipment Carryover

Line Hem Caryover

Total

$3214 \quad 22$

229

$6561 \quad 6117$

693

\begin{tabular}{rrr}
3214 & 2229 & 55 \\
0 & 0 & \\
0 & 0 & \\
0 & 0 & \\
3214 & 2229 & 556 \\
0 & 0 & \\
0 & 0 & \\
0 & 0 & \\
\hline
\end{tabular}

$\begin{array}{rrr}561 & 6117 & 693 \\ 0 & 0 & 0 \\ 0 & 0 & 0 \\ 0 & 0 & 0 \\ 5564 & 6117 & 693 \\ 0 & 0 & 0 \\ 0 & 0 & 0 \\ 0 & 0 & 0\end{array}$

$\begin{array}{r}693 \\ 0 \\ 0 \\ 0 \\ \hline 693 \\ 0 \\ 0 \\ 0 \\ \hline 693\end{array}$

0
0
0
0
0
0
0
0
0
0

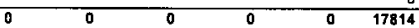

RL-WM01 6696-0 Debris Removal Project

Expense

$\begin{array}{lllll}3591 & 1117 & 4060 & 2872 & 293\end{array}$

CENRTC
Line Item

GPP

Total BCWSPMB (1) \& (2)

Expense Carryover

Capital Equipment Carryover

$3594 \quad 1117$

$2872 \quad 293$

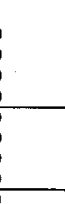

\begin{tabular}{ll}
0 & 0 \\
0 & 0 \\
0 & 0 \\
0 & 0 \\
\hline 0 & 0 \\
0 & 0 \\
0 & 0 \\
0 & 0 \\
\hline 0 & 0
\end{tabular}

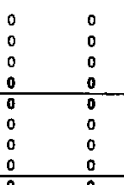

17814

Line Item Carryover

Line Item

$\begin{array}{lllll}3591 & 1117 & 4060 & 2872 & 293\end{array}$

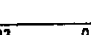


4.1.2 Budget Authority (BA) by Year by WBS by Fund Type

RL WBS 1.4.1

PAD \# ADS \# WES TTRE FUND TYPE

Expense

CENRTC

Line Item

GPP

Total BCWS/PMB (1) \& (2)

Expense Carryover

Capital Equipment Carryover

Line Item Carryover

rotal

$\begin{array}{rr}3591 & F Y 1 \\ 0 & \\ 0 & \\ 0 & \\ 3591 & 0 \\ 0 & 1197 \\ 0 & \\ 0 & \\ 3591 & 1117\end{array}$

$\begin{array}{rrrrrrr}1997 & \text { FY1998 } & \text { FY1999 } & \text { FY2000 } & \text { FY2001 } & \text { FY2002 } & \text { FY200 } \\ 117 & 4060 & 2872 & 293 & 0 & 0 & 0 \\ 0 & 0 & 0 & 0 & 0 & 0 \\ 0 & 0 & 0 & 0 & 0 & 0 \\ 0 & 0 & 0 & 0 & 0 & 0 & 0 \\ 1177 & 4060 & 2872 & 293 & 0 & 0 \\ 0 & 0 & 0 & 0 & 0 & 0 \\ 0 & 0 & 0 & 0 & 0 & 0 \\ 0 & 0 & 0 & 0 & 0 & 0 & 0 \\ 1117 & 4060 & 2872 & 293 & 0 & 0\end{array}$

RL-WM01 6696-0 Water Treatment System

Expense

Expense
CENRTC

$\begin{array}{lllll}4440 & 1502 & 1802 & 1015 & 967\end{array}$

967

Line lter

$4440 \quad 1502$

1802

Line I

GPP

14037

$8624 \quad 10$

Expense Carryover

Capital Equipment Carryover

Line Item Carryover

Total

Sub-Total Integrated Water Treatment System

Expense

CENRTC

Line lte

GPP

Total BCWS/PMB (1) \& (2)

Expense Caryover

Capital Equipment Carryover

Line Item Carryover

$430 \quad 430$

96

Tota

Total Facility Projects

\begin{tabular}{lrr} 
Expense & 34400 & $\mathbf{2 2 1 9}$ \\
CENRTC & 1422 & 1101 \\
Line Item & 0 & \\
GPF & 0 & \\
\hline Total BCWS/PMB (1) \& (2) & $\mathbf{3 5 8 2 2}$ & $\mathbf{3 3 2 1}$ \\
Expense Caryover & -735 & 735 \\
Capital Equipment Carryover & -430 & 430 \\
Line Item Carnover & 0 & \\
\hline Total & $\mathbf{3 4 6 5 7}$ & $\mathbf{3 4 3 7}$
\end{tabular}

\begin{tabular}{rrrrr} 
& FY2004 & FY2005 & FY2006 & TOTAL \\
\hline 0 & 0 & 0 & 0 & 11933 \\
0 & 0 & 0 & 0 & 0 \\
0 & 0 & 0 & 0 & 0 \\
0 & 0 & 0 & 0 & 0 \\
\hline 0 & 0 & 0 & 0 & 11933 \\
0 & 0 & 0 & 0 & 0 \\
0 & 0 & 0 & 0 & 0 \\
0 & 0 & 0 & 0 & 0 \\
\hline 0 & 0 & 0 & 0 & 11933
\end{tabular}

RL-WM01 6696-0 MCO ACquisition Definition

Expense

CENRTC

Line iter

Total BCWS/PMB (1) \& (2)

Expense Carryover

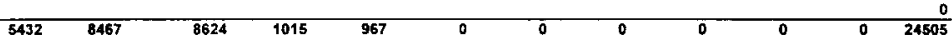

Capital Equipment Carryover

Line Item Camyover

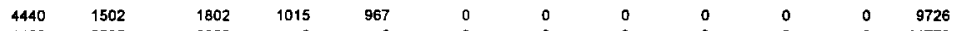

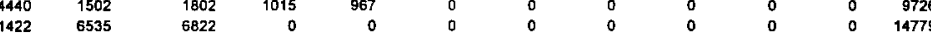

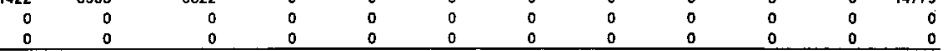

Total

$1695 \quad 19$

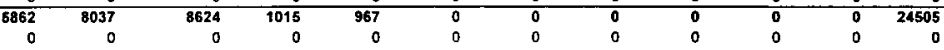

$-430 \quad 430$

\begin{tabular}{rrr}
30 & 0 & 0 \\
0 & 0 & 0 \\
\hline 67 & 8624 & 1015
\end{tabular}

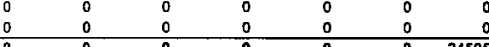

$\begin{array}{rrrrrllllll}197 & 19649 & 11372 & 2806 & 250 & 0 & 0 & 0 & 0 & 0 & 90674 \\ 013 & 9072 & 0 & 0 & 0 & 0 & 0 & 0 & 0 & 0 & 21507\end{array}$

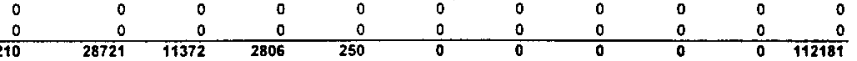

$\begin{array}{lll} & 0 & 0 \\ 0 & 0 & 0 \\ & 0 & 0\end{array}$

0

0

0 


\section{SPENT NUCLEAR FUEL PROJECT \\ WBS 1.4.1}

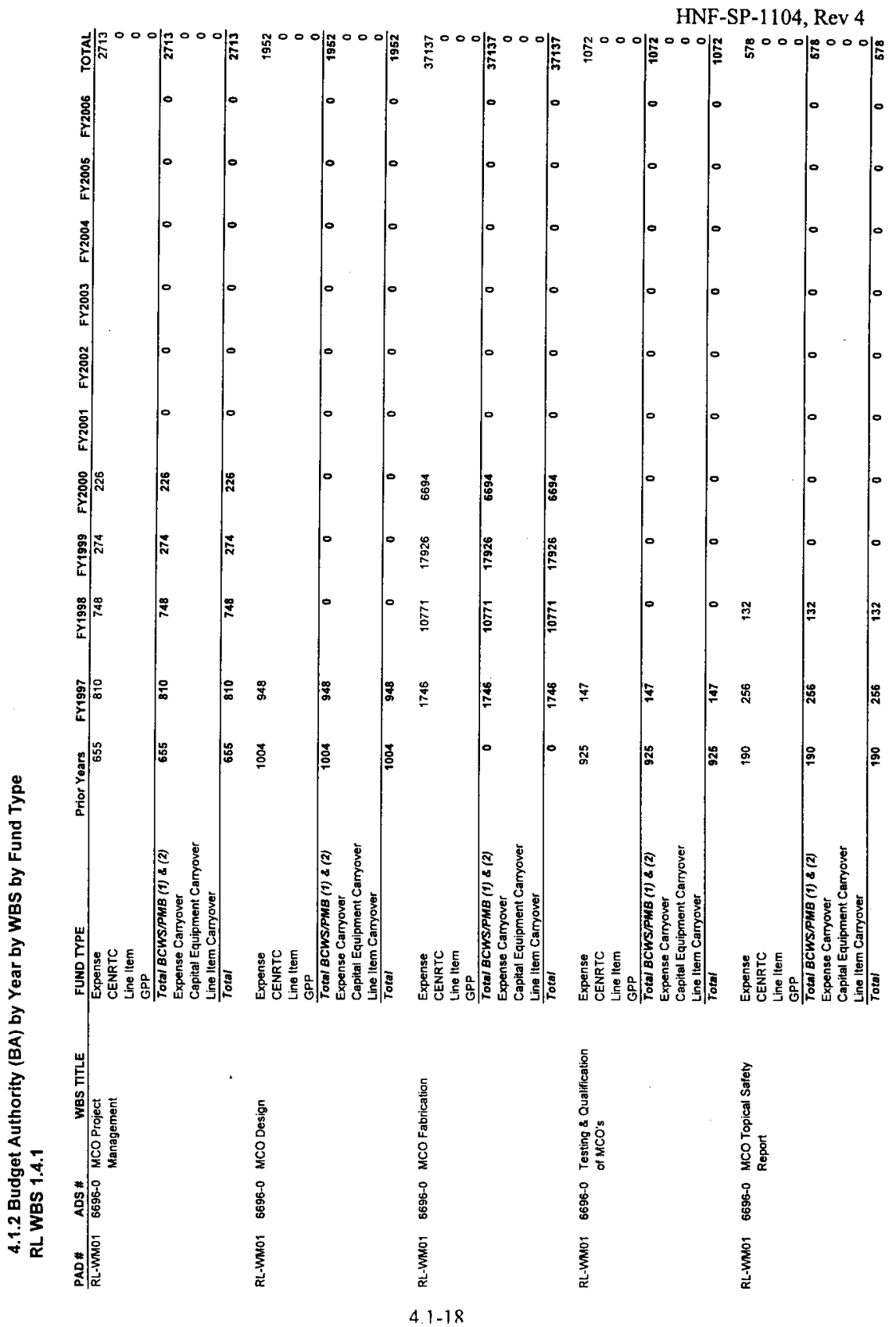


4.1.2 Budget Authority (BA) by Year by WBS by Fund Type

RL WBS 1.4.1

PAO \# ADS\# WBS TITLE Sub-Total Muti Canister Overpacks (MCOs)

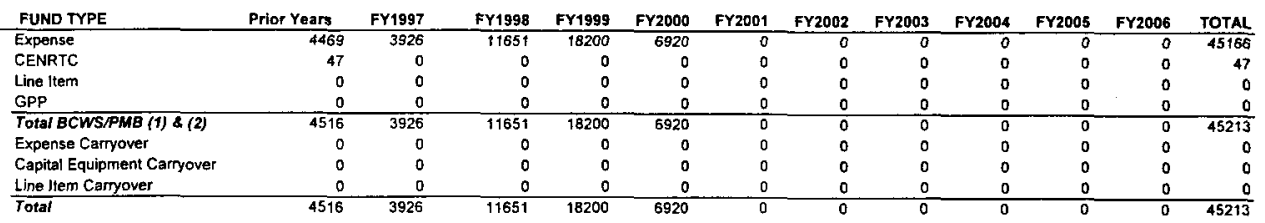

RL-WMO1 6696-0 CaskTransportation Acquisition Definition

Expense

1818

CENRTC

Line Item

LPP Item

GPP

Expense Caryover

Capital Equipment Cartyover

Line Item Carryover

Total

RL-WMO1 6696-0 Cask/Transportation Project Management

Expense

CENRTC

Line tern

GPP

Total BCWS/PMB (1) \& (2)

Expense Carryover

Capital Equipment Carryover

Line Item Carryover

Total
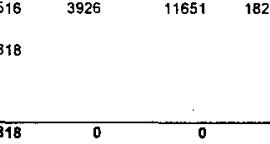

6920

1818
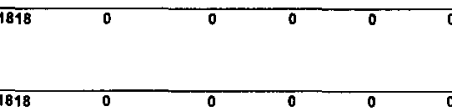

Expense

CENRTC

Line Item

GPP Design

RL-WM01 6696-0 CaskTransportation Fabrication

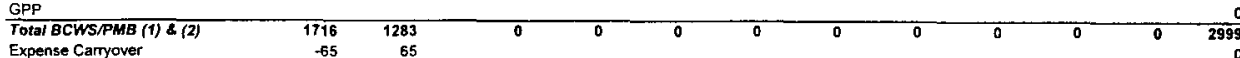

Capital Equipment Carryover $\quad-640 \quad 640 \quad 000$

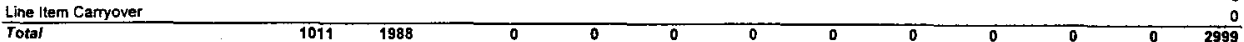

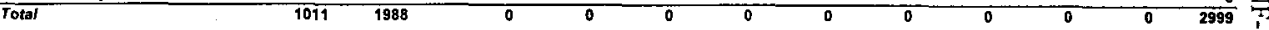

Expense

CENRTC

783
4724

0 ?

Line Item

3010

8517

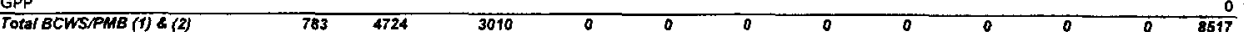

(1)

Capital Equipment Camyover

Line Item Carryover

$\mathbf{7 8 3} \quad \mathbf{4 7 2 4} \quad \mathbf{3 0 1 0}$

3010

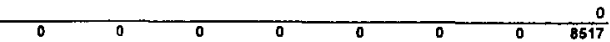




\section{SPENT NUCLEAR FUEL PROJECT WBS 1.4.1}

HNF-SP-1 104, Rev 4

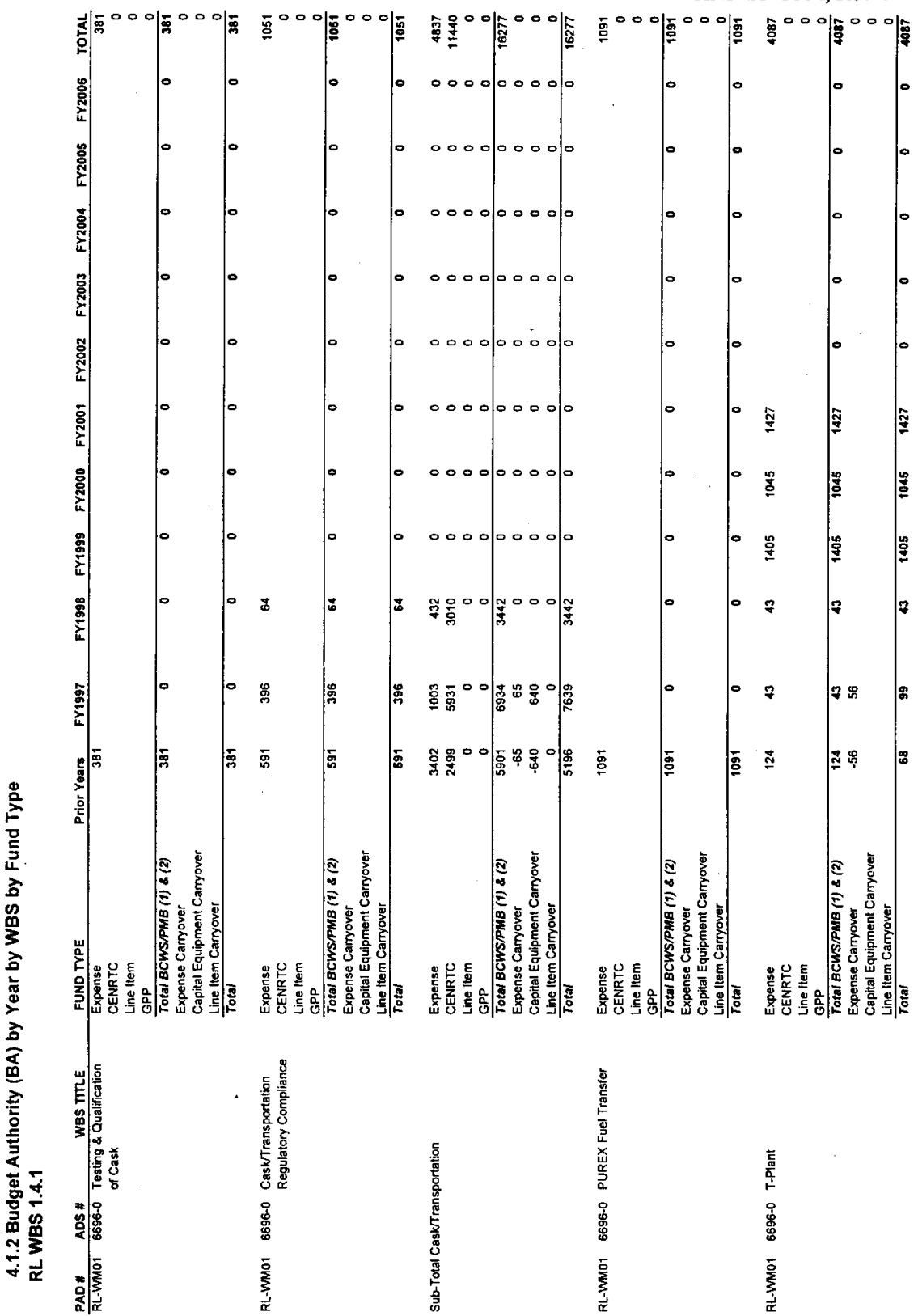


4.1.2 Budget Authority (BA) by Year by WBS by Fund Type

RL WBS 1.4.1

PAD : ADS

WBS TITLE $\quad$ FUND TYPE

Expense

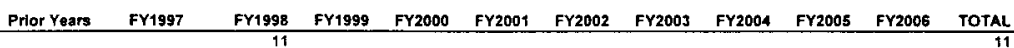

Line Item

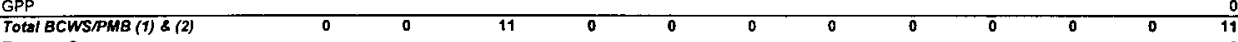

Expense Carryover

Capital Equipment Carryove

Line Item Carryover

Total

Expense

CENRTC

Line

GPP

325, 327

$\rightarrow$ RL-WMOt 6696-0 308 Annex

Expense Carryover

Capital Equipment Carryove

Line Item Carryover

Total

Expense

CENRTC

Line Item

GPP

Expense Carryover

Capital Equipment Carryover

Line ltem Carryover

Total

0

110

Expense

CENRTC

Line ltem

$249 \quad 3$

1396

96

RL-WMO1 6696-0 FFTF

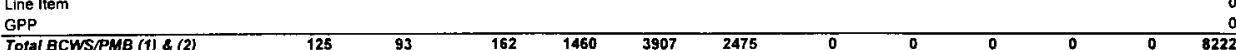

$\begin{array}{lllllllllllll}\text { Expense Carryover } & 125 & 93 & 162 & 1460 & 3907 & 2475 & 0 & 0 & 0 & 0 & 0 & 8222\end{array}$

Capital Equipment Carryover

Line Item Carryover

0 T

Total

$85 \quad 133$

Expense

CENRTC

Line ltem

GPP

Total BCWS/PMB (1) \& (2)

$14 \quad 27$

$\begin{array}{llll}142 & 1460 & 3907 & 2475 \\ 38 & 28 & 103 & 29\end{array}$

Expense Carryover

Capital Equipment Carryover

Line ltem Carryover

Total

29

14

27

$\begin{array}{lll}38 & 28 & 103\end{array}$

29 
4.1.2 Budget Authority (BA) by Year by WBS by Fund Type

RL. WBS 1.4.1

PAD \# ADS \# WES TITL RL-WMO1 6696-0 N Reactor Fuel Transfer

FUND TYPE Prior Years FYt997 FY1998 FY1999 FY2000 FY2001 FY2002 FY2003 FY2004 FY2005 FY2006 TOTAL CENRTC

Line Iter GPP

$\begin{array}{llllllll}\text { Total ACWS/PMB (1) \& (2) } & 24 & 0 & 0 & 0 & 0 & 0 & 0\end{array}$

Total BCWS/PMB (1) \& (2)

Expense Carryover

Capital Equipment Carryover

Line Item Carryover

Total

RL-WM01 6696-0 National Programs

Expense

CENRTC

Line Item

Line Item
GPP

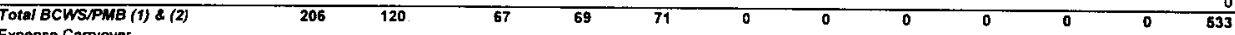

Expense Carryover

Capital Equipment Carryover

Line Item Carryover

Total

$\perp$ Sub-Total Other Hanford Fuel

Expense

Line Item

GPP

Total BCWS/PMB (1) \& (2)

Expense Carryover

Capital Equipment Carryover

Line Item Carryover

Total

Total SNF Storage

Expense

CENRTC

Line Item

GPP

Total BCWS/PMB (1) \& (2)

Expense Caryover

Capital Equipment Carryove

Line Item Carryover

Total

$\begin{array}{rr}206 & 120 \\ 2363 & 47 \\ 0 & \\ 0 & \\ 0 & \\ 2363 & 47 \\ -272 & 272 \\ 0 & 0 \\ 0 & 0 \\ 2091 & 74\end{array}$

RL-WM01 6696-1 CSB Project Management

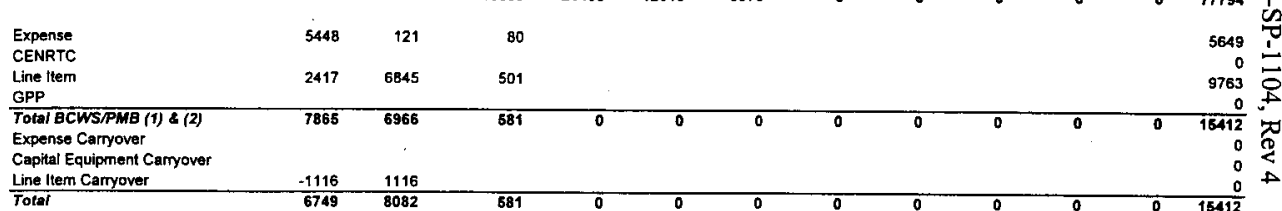


4.1.2 Budget Authority (BA) by Year by WBS by Fund Type

RL WBS 1.4.1

PAD \# ADS \#

WBS TIILE

FUND TYPE

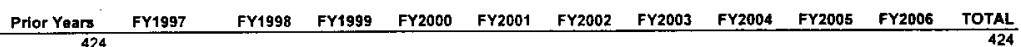

RL-WMO1 6596-1 CSB Design

Expense

424

$\begin{array}{llll}11897 & 4075 & 338 & \end{array}$

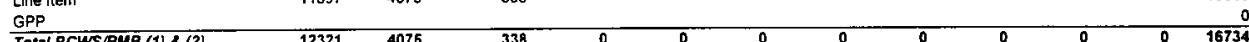

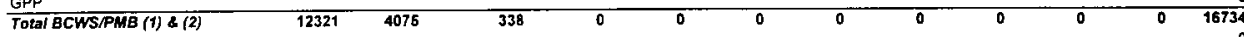

Expense Carryove

Capital Equipment Carryover

Line Item Carnyover

Total

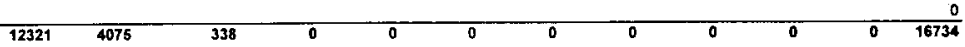

RL-WM01 6696-1 CSB Construction

\section{Expense}

CENRTC

Line Item

$55 \quad 188 \quad 44$

GPP

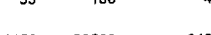

44

287

Total

$24452 \quad 33503$

0428

24507 33691

6472

Expense Carryover

Capital Equipment Carryover

$\begin{array}{r}-9781 \quad 9781 \\ \hline 14726\end{array}$

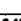

\section{Expense}

EXpense

CENRTC

Line tte

Total BCWSTPM (1) \& (2)

1134

45

Expense Caryover

Capital Equipment Carryover

Line item Carryover

Total

RL-WM01 6696-1 MHM Fabrication

Expense

Line lten

GPP

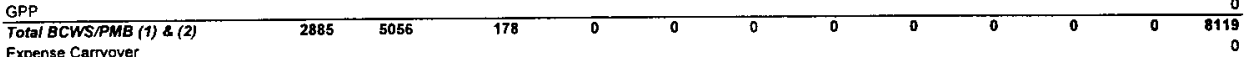

Expense Carryover

Capital Equipment Carryover

113420

28

Line Item Carryover

Total

Total Canister Storage Building

\begin{tabular}{|c|c|c|c|c|c|c|c|c|c|c|c|c|}
\hline Expense & 6352 & 413 & 141 & 0 & 0 & 0 & 0 & 0 & 0 & 0 & 0 & 6906 \\
\hline CENRTC & 2460 & 4952 & 161 & 0 & 0 & 0 & 0 & 0. & 0 & 0 & 0 & 7573 \\
\hline Line Item & 39900 & 45051 & 7312 & 0 & 0 & 0 & 0 & 0 & 0 & 0 & 0 & 92263 \\
\hline GPP & 0 & 0 & 0 & 0 & 0 & 0 & 0 & 0 & 0 & 0 & 0 & 0 \\
\hline Total BCWS/PMB (1) \& (2) & 48712 & 50416 & 7614 & 0 & 0 & 0 & 0 & 0 & 0 & 0 & 0 & 106742 \\
\hline Expense Carryover & 0 & 0 & 0 & 0 & 0 & 0 & 0 & 0 & 0 & 0 & 0 & 0 \\
\hline Capital Equipment Carryover & -9673 & 1673 & 0 & 0 & 0 & 0 & 0 & 0 & 0 & 0 & 0 & 0 \\
\hline Line Item Carryover & -10897 & 10897 & 0 & 0 & 0 & 0 & 0 & 0 & 0 & 0 & 0 & 0 \\
\hline Total & 36142 & 62986 & 7614 & 0 & 0 & 0 & 0 & 0 & 0 & 0 & 0 & $106 \overline{742}$ \\
\hline
\end{tabular}




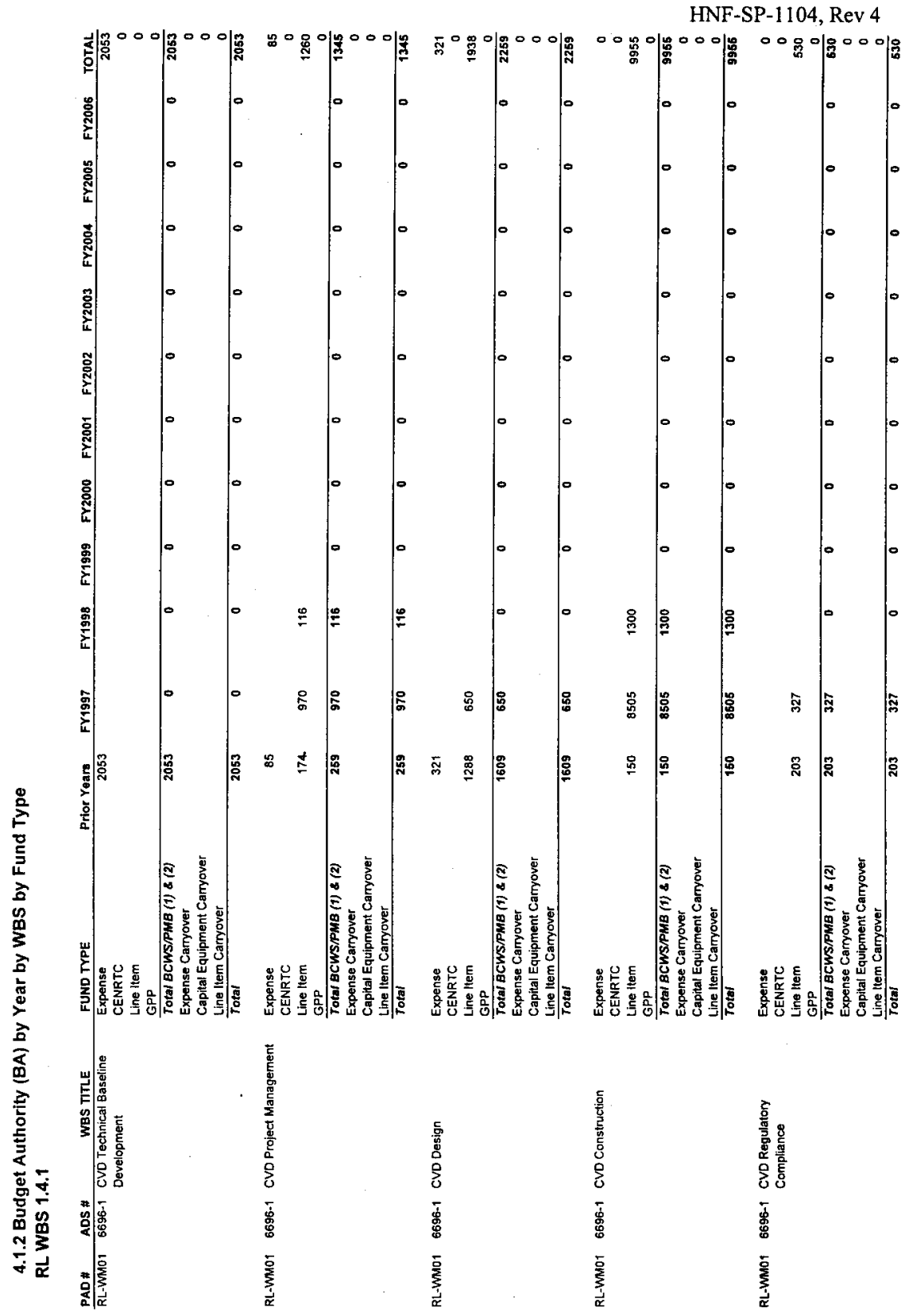


4.1.2 Budget Authority (BA) by Year by WBS by Fund Type

RL WBS 1.4.1

\begin{tabular}{|c|c|c|c|c|c|c|c|c|c|c|c|c|c|}
\hline$\frac{\text { PAD \# ADS \# }}{\text { Sub-Total Cold Vacuum Drying }}$ & FUND TYPE & Prior Years & FY1997 & FY1998 & FY1999 & FY2000 & FY2001 & FY2002 & FY2003 & FY2004 & FY2005 & FY2006 & TOTAL \\
\hline \multirow[t]{9}{*}{ Sub-Total Cold Vacuum Drying } & Expense & 2459 & 0 & $\overline{0}$ & 0 & 0 & 0 & 0 & D & 0 & 0 & 0 & 2459 \\
\hline & CENRTC & 0 & 0 & 0 & 0 & 0 & 0 & 0 & 0 & 0 & 0 & 0 & 0 \\
\hline & Line ttem & 1815 & 10452 & 1416 & 0 & 0 & 0 & 0 & 0 & 0 & 0 & 0 & 13683 \\
\hline & GPP & 0 & 0 & 0 & 0 & 0 & 0 & 0 & 0 & 0 & 0 & 0 & 0 \\
\hline & Total BCWS $/ P M B(1) \&(2)$ & 4274 & 10452 & 1416 & 0 & 0 & $\overline{0}$ & $\overline{0}$ & 0 & 0 & 0 & 0 & 16142 \\
\hline & Expense Carryover & 0 & 0 & 0 & 0 & 0 & 0 & 0 & 0 & 0 & 0 & 0 & 0 \\
\hline & Capital Equipment Carryover & 0 & 0 & 0 & 0 & 0 & 0 & 0 & 0 & 0 & 0 & 0 & 0 \\
\hline & Line Item Carryover & 0 & 0 & 0 & 0 & 0 & 0 & 0 & 0 & 0 & 0 & 0 & 0 \\
\hline & Total & 4274 & 10452 & 1416 & 0 & 0 & 0 & 0 & 0 & 0 & 0 & 0 & 16142 \\
\hline
\end{tabular}

RL-WM01 6696-1 HCS ACquisition Definition

芩 RL-WM01 6696-1 HCS Project Management

Expense

$966 \quad 201$

Line Item

GPP

Total BCWS/PMB (1) \& (2)

Expense Carryover

Capital Equipment Carryover

Line Item Caryover

Total

201

1

o
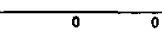

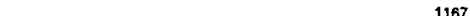

966

Expense

CENRTC

Line Item

73

GPP

$94 \quad 1720 \quad 1288$

288

Expense Carryover

$167 \quad 172$

1288

Capital Equipment Carryover

Line Item Carryover

Line Itern Cam

167

1286

\section{Expense}

CENRTC

Line ltem

296

Line Item

$1166 \quad 2833$

$\begin{array}{lll}\text { GPP } & \\ \text { Total BCWS/PMB (1) \& (2) } & 1462 & 2833\end{array}$

Capital Equipment Carryover

Line Item Carryover

$115 \quad 115$

1288

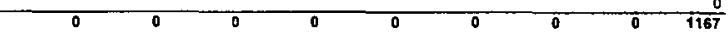

Total

$1347 \quad 2948$

Expense

CENRTC

Line Item
GPP

$25 \quad 123 \quad 6502$

o

0 Z

RL-WM01 6696-1 HCS Construction

$25 \quad \overline{123} \quad 6502$

$502+6$

Expense Caryover

Capital Equipment Carryover

Line Item Carryover

$25-123$

6502

0

01

官

$0 \$$

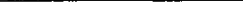


4.1.2 Budget Authority (BA) by Year by WBS by Fund Type

RL WBS 1.4.1

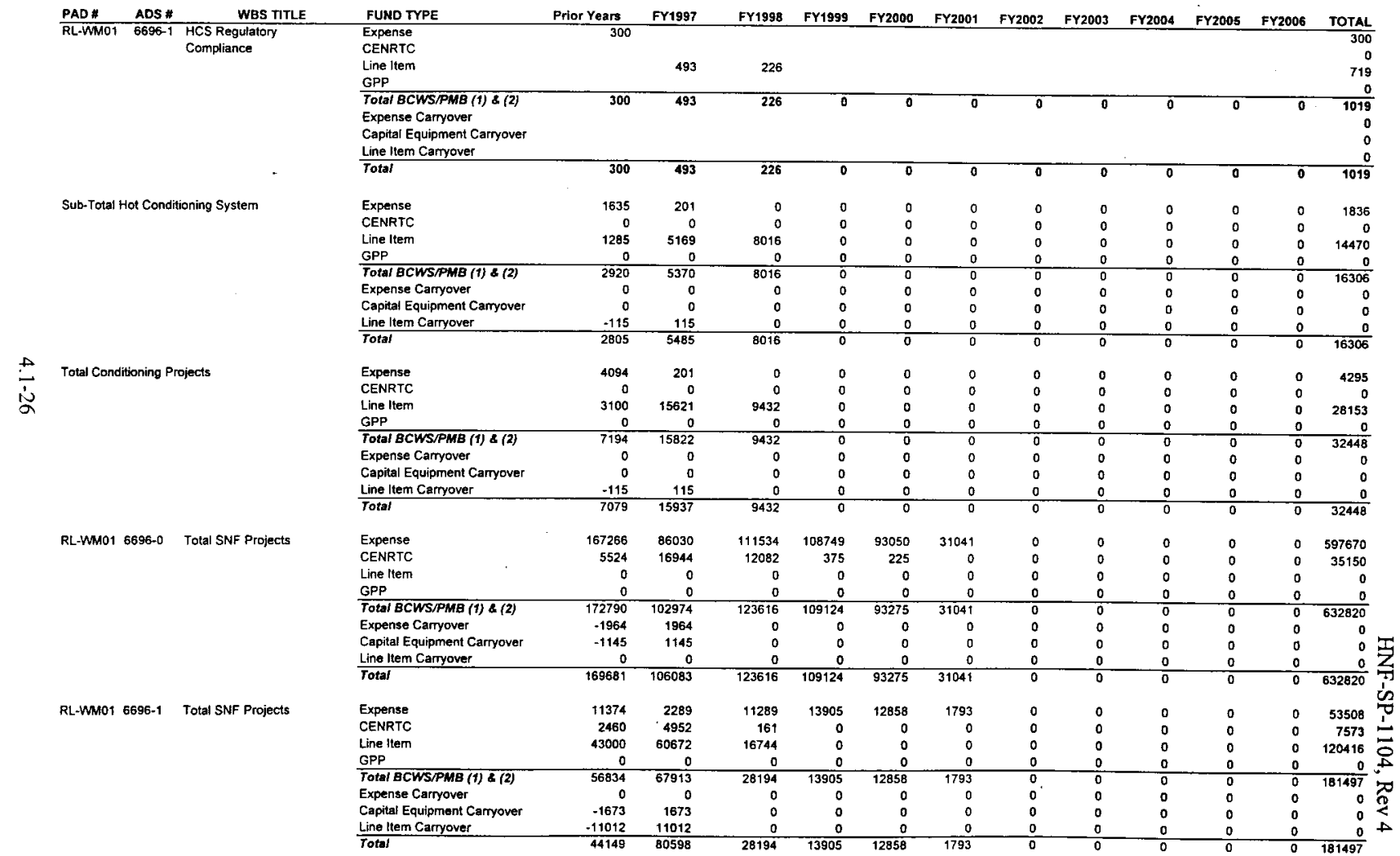




\section{SPENT NUCLEAR FUEL PROJECT WBS 1.4.1}

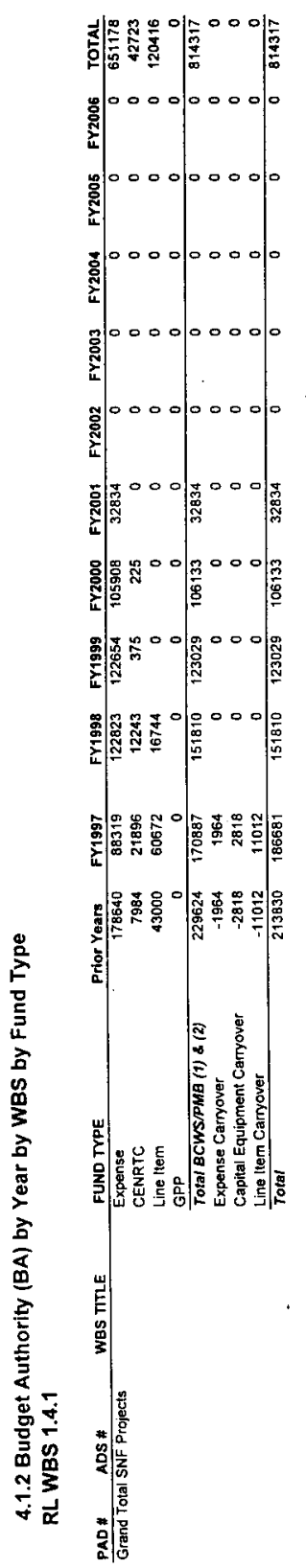

HNF-SP-1104, Rev 4 
4.1.3 Cost Baseline (BO) by Year by WBS

RL WBS 1.4.1

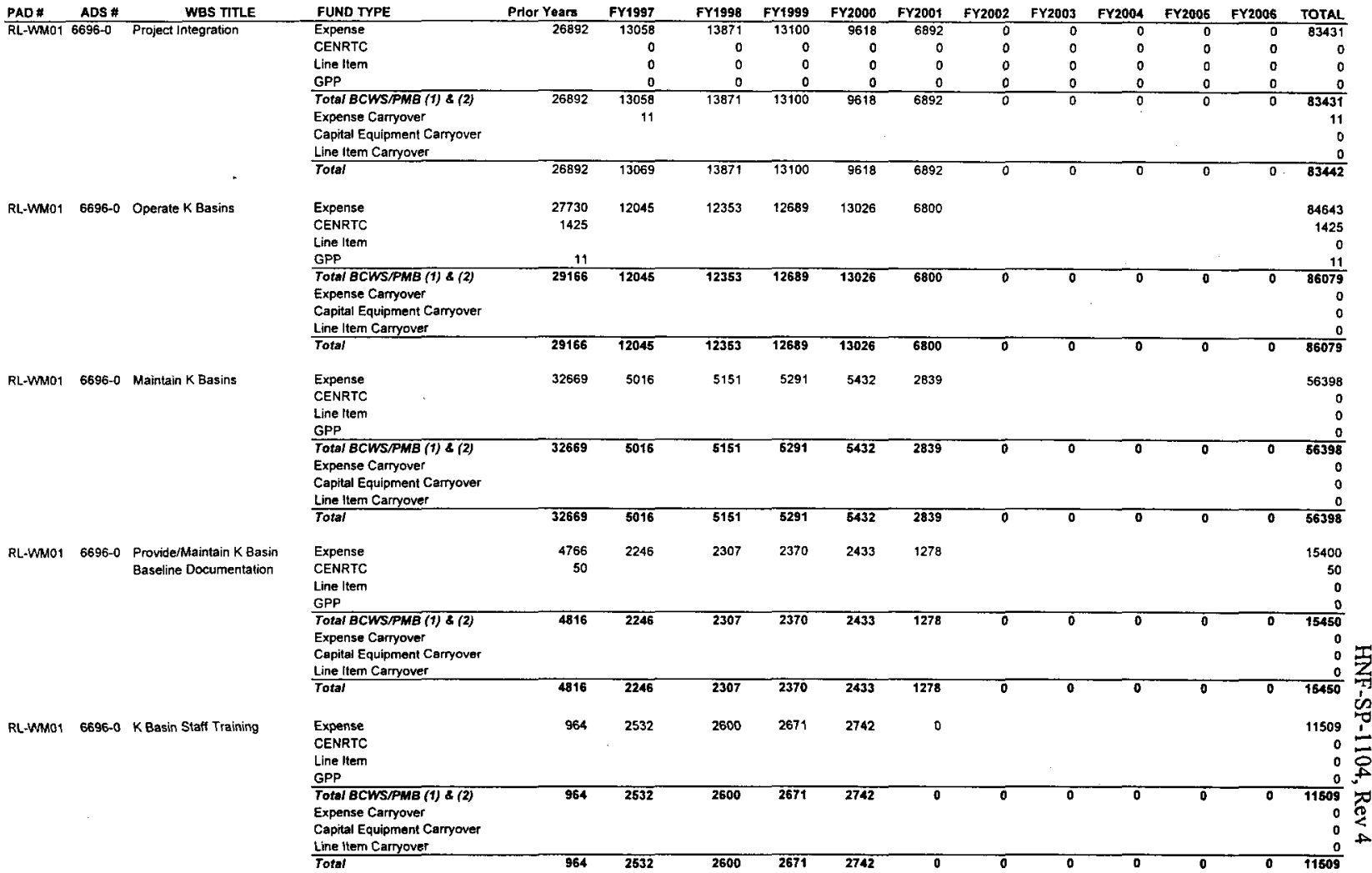

(1) Budgated Cost of Work Schedused (BCWS) equals Performance Meesurement Baseline (PMB) 


\subsubsection{Cost Baseline (BO) by Year by WBS}

\section{RL WBS 1.4.1}

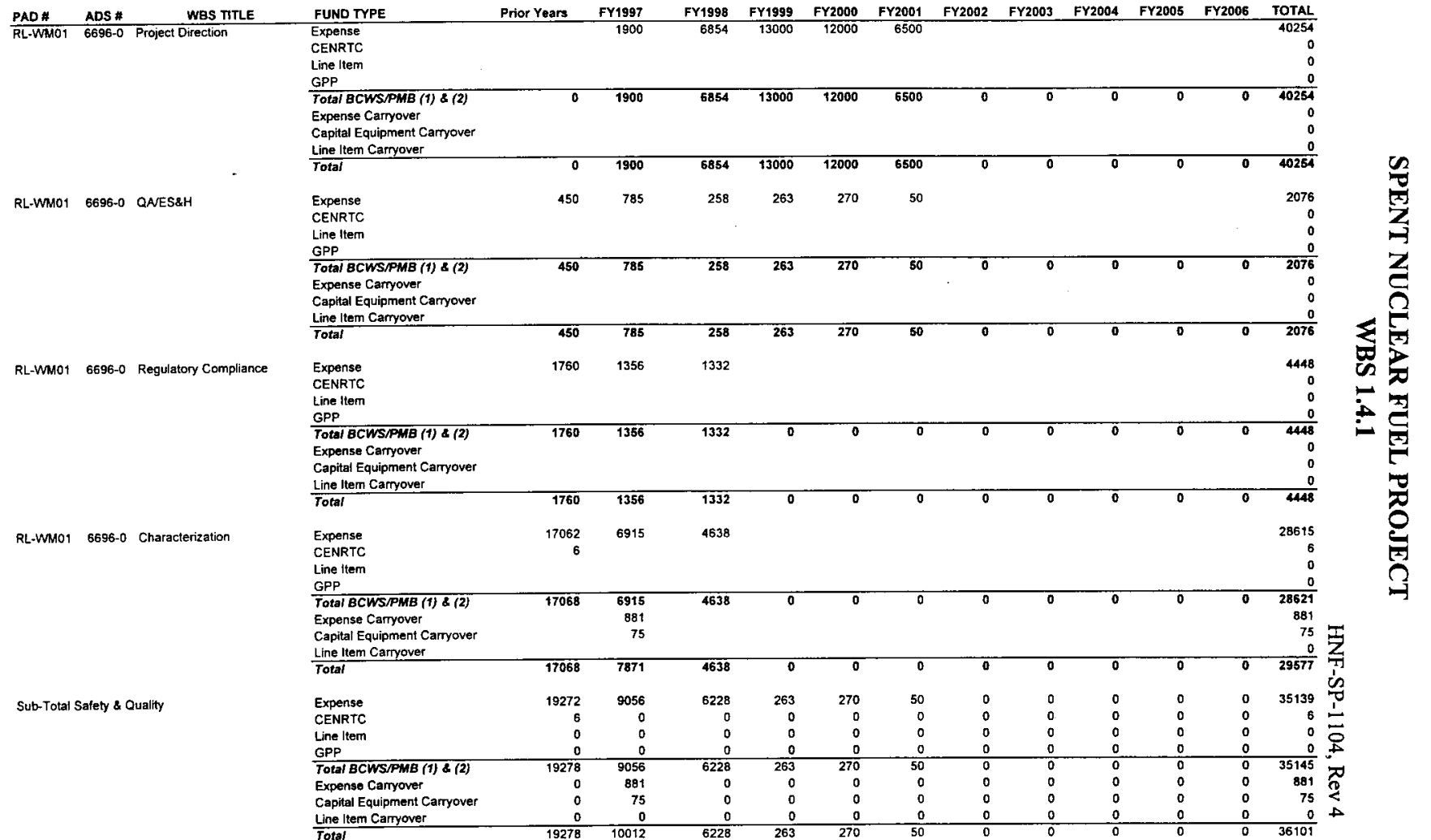




\section{SPENT NUCLEAR FUEL PROJECT \\ WBS 1.4.1}

HNF-SP-1 104, Rev 4

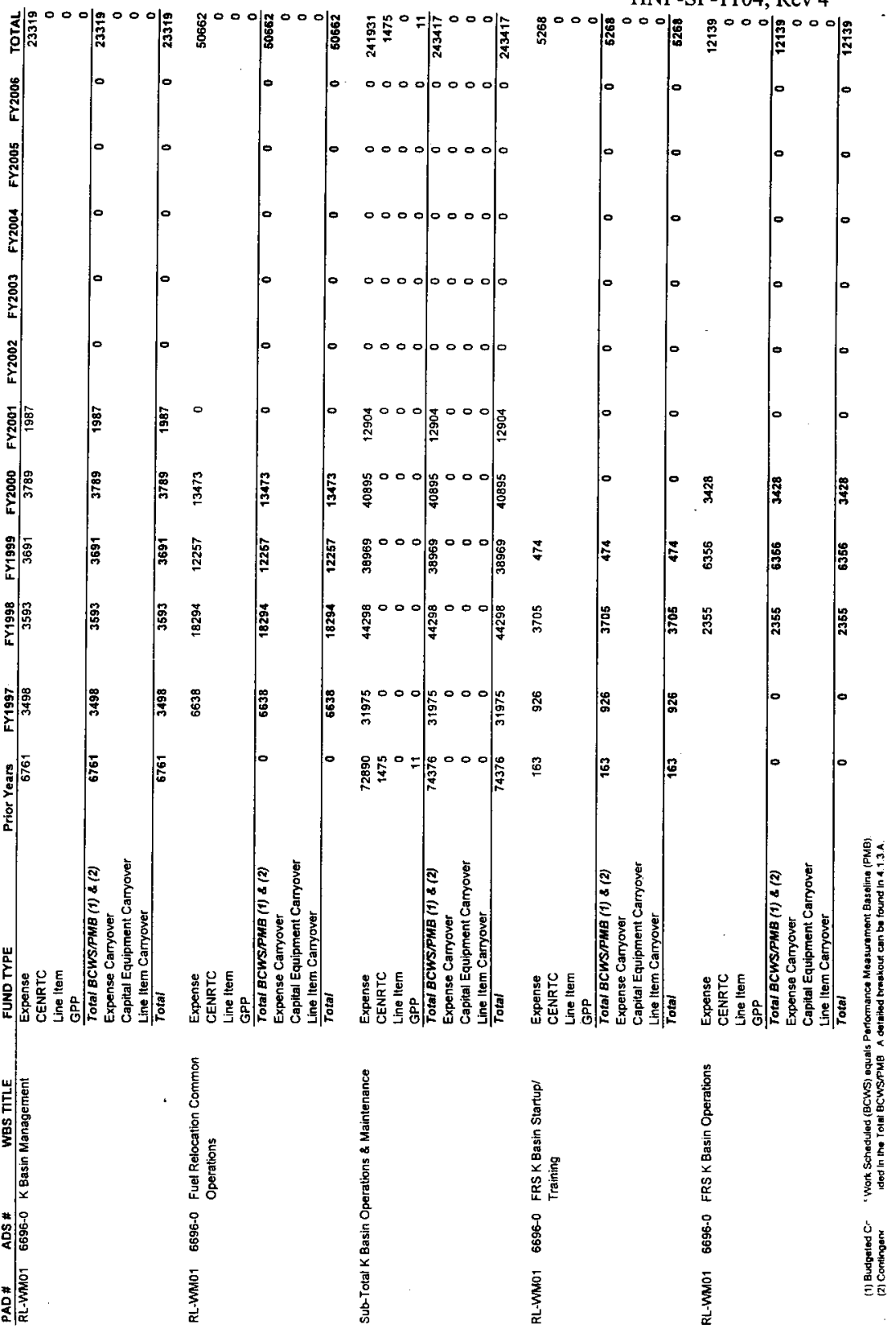


4.1.3 Cost Baseline (BO) by Year by WBS RL WBS 1.4.1

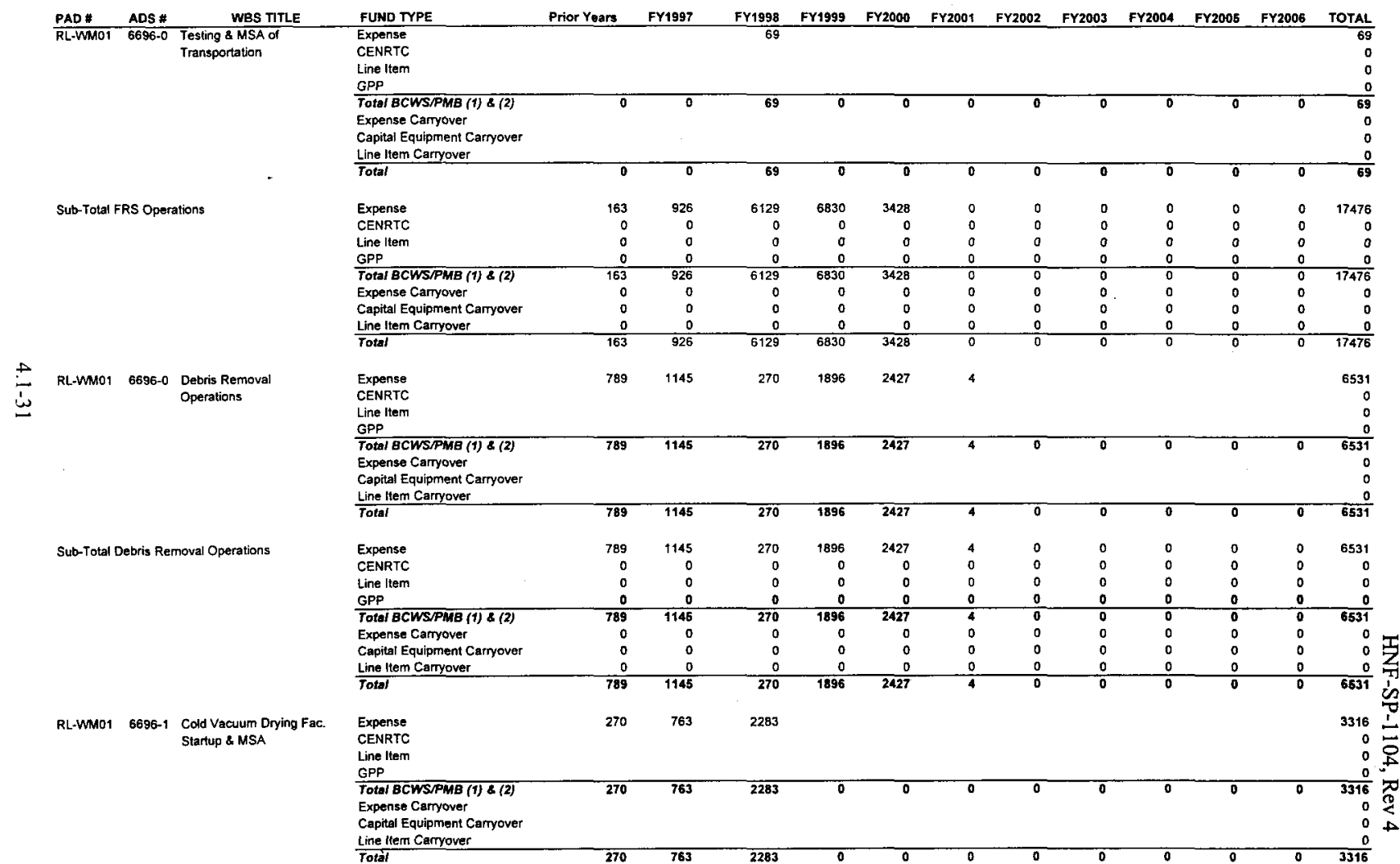


4.1.3 Cost Baseline (BO) by Year by WBS

RL WBS 1.4.1

PAD* ADS * FUND TYPE Expense

CENRTC

Line ltem

TPP.

Expense Carryover

Capital Equipment Carryover

Line ltem Caryover

Total

Sub-Total CVO Operations

RL-WMO1 6696-0 Water Treatment

\begin{tabular}{lrrr} 
Expense & 270 & 763 & 40 \\
CENRTC & 0 & 0 & \\
Line Item & 0 & 0 & \\
GPP & 0 & 0 & \\
\hline Total BCWSRPMB (1) \& (2) & 270 & 763 & 40 \\
Expense Cartyover & 0 & 0 & \\
Capital Equipment Carryover & 0 & 0 & \\
Line liem Camyover & 0 & 0 & \\
\hline Total & & 0
\end{tabular}

\begin{tabular}{rrr}
$\mathbf{1 7 4 7}$ & $\mathbf{5 4 7 1}$ & $\mathbf{4 6 9 5}$ \\
$\mathbf{4 0 3 0}$ & $\mathbf{5 4 7 1}$ & $\mathbf{4 6 9 5}$ \\
0 & 0 & 0 \\
0 & 0 & 0 \\
0 & 0 & 0 \\
\hline 4030 & 5471 & $\mathbf{4 6 9 5}$ \\
0 & 0 & 0 \\
0 & 0 & 0 \\
0 & 0 & 0 \\
\hline 4030 & 5471 & 4695
\end{tabular}

Total

Expense

$\begin{array}{lllll}965 & 373 & 1646 & 1239 & 6351\end{array}$

CENRTC

Line

51

Total BCWS/PMB (1) \& (2)

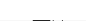

351

Expense Carryover

Capital Equipment Carryover

Line ltem Carryover

Line liem Carryover

96

373

$1646-9239$

0

-

$+2$

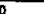

Expense

CENRTC

Lime them

GPP

Total BCW5/PMB (1) \& (2)

Expense Carryover

Cepital Equipment Carryove

Line ltem Carryover

Tota

$\begin{array}{lllll}\text { Expense } & 0 & 960 & 2861 & 479\end{array}$

RL-WM01 6696-0 Sludge Removal Operations

CENRTC

Line ltem

GPP

Total BCWS/PMB (1) \& (2)

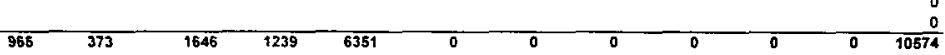

Capital Equipment Carryover

Line Item Carryover

\begin{tabular}{rrr}
$\mathbf{9 6 5}$ & $\mathbf{3 7 3}$ & 16 \\
0 & 0 & \\
0 & 0 & \\
0 & 0 & \\
$\mathbf{9 6 5}$ & $\mathbf{3 7 3}$ & $\mathbf{1 6}$ \\
0 & 0 & \\
0 & 0 & \\
0 & 0 & \\
\hline $\mathbf{9 6 5}$ & $\mathbf{3 7 3}$ & $\mathbf{1 6}$
\end{tabular}

$\begin{array}{rrr}1646 & 1239 & 6 \\ 0 & 0 & \\ 0 & 0 & \\ 0 & 0 & \\ 1646 & 1239 & 6 \\ 0 & 0 & \\ 0 & 0 & \\ 0 & 0 & 0 \\ 1646 & 1239 & 6\end{array}$

\begin{tabular}{rl}
6351 & 0 \\
0 & 0 \\
0 & 0 \\
0 & 0 \\
\hline 6351 & 0 \\
0 & 0 \\
0 & 0 \\
0 & 0 \\
\hline 6351 & 0 \\
\hline
\end{tabular}

\begin{tabular}{ll}
0 & 0 \\
0 & \\
0 & \\
0 & 0 \\
0 & 0 \\
0 & 0 \\
0 & 0 \\
0 & 0 \\
\hline 0 & 0
\end{tabular}

\begin{tabular}{l}
0 \\
0 \\
0 \\
0 \\
\hline 0 \\
0 \\
0 \\
0 \\
\hline 0
\end{tabular}

\begin{tabular}{l}
0 \\
0 \\
0 \\
0 \\
\hline 0 \\
0 \\
0 \\
0 \\
\hline 0
\end{tabular}


4.1.3 Cost Baseline (BO) by Year by WBS

RL WBS 1.4.1

PAD* ADS * WBS TITLE

Sub-Total Sludge Removal Operations

FUND TYPE

Prior Years

$\begin{array}{lrr} & \text { FY1997 } & \text { FY1998 } \\ 0 & 0 & \\ 0 & 0 & \\ 0 & 0 & \\ 0 & 0 & \\ 0 & 0 & \\ 0 & 0 & \\ 0 & 0 & \\ 0 & 0 & \\ 0 & 0 & \end{array}$

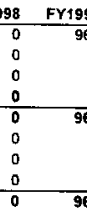

RL-WM01 6696-1 Hot Cond Sys Startup and $O R R$

\begin{tabular}{l} 
Expense \\
CENRTC \\
Line Item \\
GPP \\
\hline Total BCWS/PMB (1) \& (2) \\
Expense Carryover \\
Capital Equipment Carryover \\
Line Item Carryover \\
Total
\end{tabular}
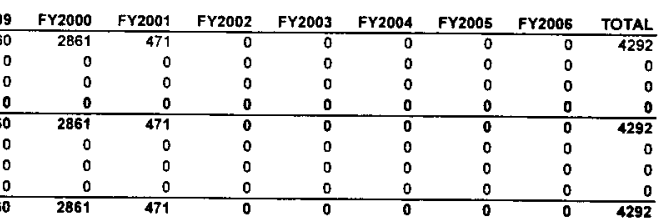

\section{Expense}

CENRTC

Line Item

$2192 \quad 403$

GPP

03

Total BCWSTPMB (1) \& (2)

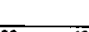

Expense Carryover

Capital Equipment Carryover

Line Item Carryover

$$
\text { Total }
$$$$
2192
$$

RL-WMO1 6696-1 HCS Operations

Expense

CENRTC

Line ltem

GPP

Total BCWS/PMB (1) \& (2)

Expense Carryover

Capital Equipment Carryover

Line Item Caryover

Line

Sub-Total HCS Operations

Expense

CENRTC

Line ltem

Total BCWS/PMB (1) \& (2)

Expense Carryover

Capital Equipment Carryover

Line Item Carryover

Total

Expense

CENRTC

CENRTC

Line lter

Total BCWS/PMB (1) \& (2)

Expense Camyover

Capital Equipment Carryover

Line Item Carryover

Total

0

$707 \quad 1333 \quad 628$

2688

0

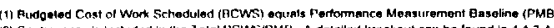

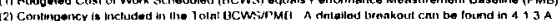




\section{SPENT NUCLEAR FUEL PROJECT WBS 1.4.1}

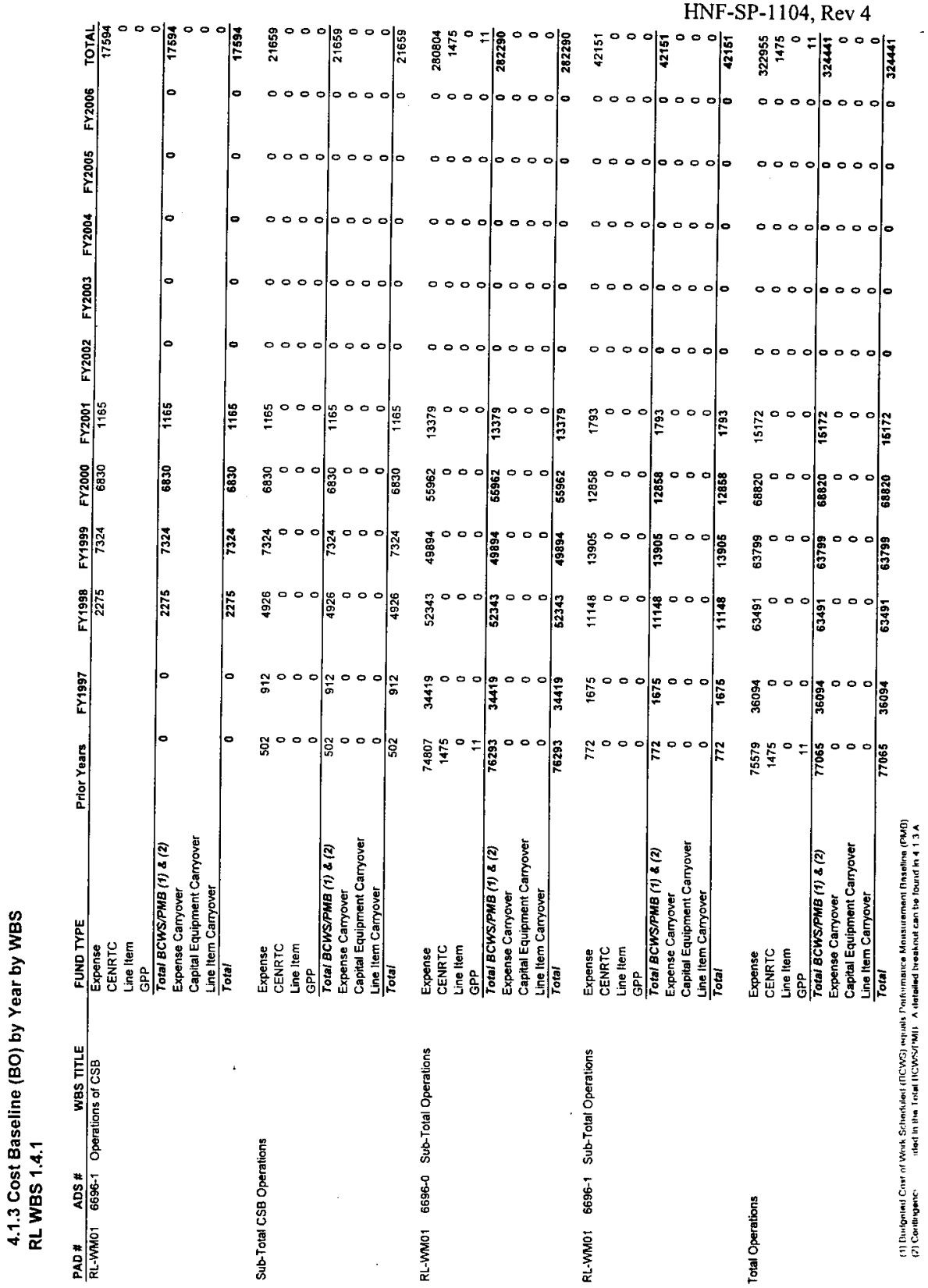




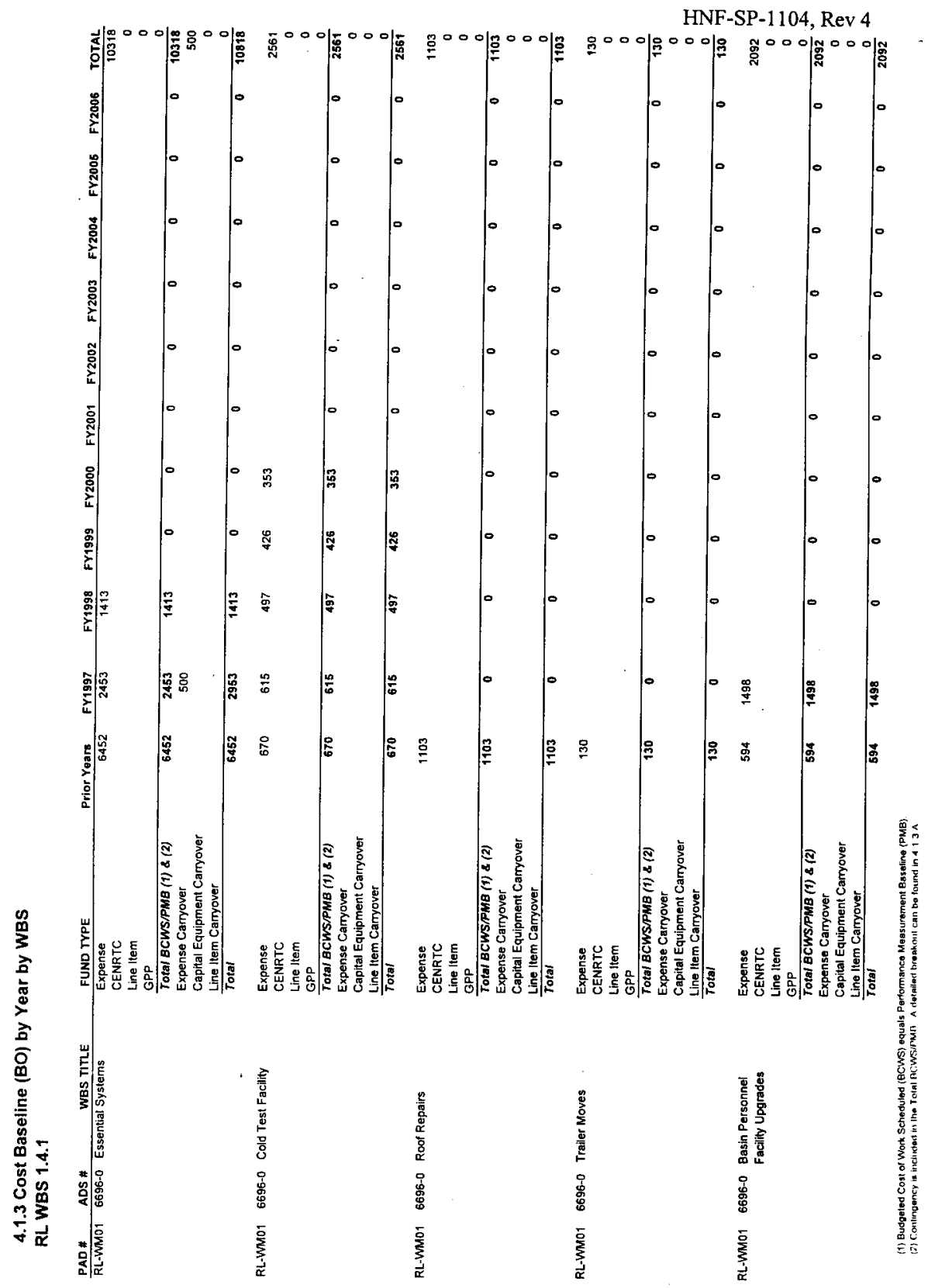


4.1.3 Cost Baseline $(B O)$ by Year by WBS

RL WBS 1.4.1

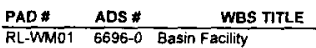
Basin Facility
Support Upgrades

$\frac{\Delta}{2}$ CENR

RL-WM09 6696-0 Dose Reduction System

RL-WM01 6696-0 Basin Facility Upgrades For Transport
FUND TYPE Expense CENRTC

Line Itern

\section{Total BCWSPMB (t) \& (2)}

Expense Carryover

Capital Equipment Carryover

Line Item Carryover

Total

\section{Expense}

CENRTC

Line Itern

GPP

Total BCWS/PMB (1) \& (2)

Expense Carryover

Capital Equipment Cartyover

Line Item Carryover

$$
\text { Totai }
$$

Expense

Line lte

GPP

Total BCWS/PMB (t) \& (2)

Expense Caryover

Capital Equipment Carryover

Line tem Carryover

Total

\section{Expense}

CENRTC

Line item

GPP
Total BCWS $F M B$ (1) \& (2)
Expense Caryover

Capital Equipment Camyover

Line liem Carryover

Total

Sub-Total Facility Ugprades

Expense
Prior Years FY1997 FY199 FY1999 FY2000 101

101

34

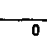

$101-34$

$5983 \quad 2041$

661

$5983-2041$

661

$+2$

0

-

0

0

$\overline{2041}$

1052

$4478 \quad 2250$

61

0

0

$\overline{0}$

0

0

CENRTC

Line ltem

GPP

ACWS $F \overline{M B}(1) \&(2)$

Expense Cartyover

Capital Equipment Carryover

Line Item Carryover

Total

-

250

o

1052

4576

2250

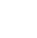

25

250

0

o

$\overline{0}$

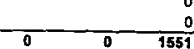

0

1551

0

0

0

$\begin{array}{rrrrrrrrrrrrr}0 & 51 & 250 & 500 & 500 & 250 & 0 & 0 & 0 & 0 & 0 & 1551 \\ 16095 & 6755 & 2821 & 926 & 853 & 250 & 0 & 0 & 0 & 0 & 0 & 27700 \\ 0 & 4478 & 2250 & 0 & 0 & 0 & 0 & 0 & 0 & 0 & 0 & 6728 \\ 0 & 0 & 0 & 0 & 0 & 0 & 0 & 0 & 0 & 0 & 0 & 0 \\ 0 & 0 & 0 & 0 & 0 & 0 & 0 & 0 & 0 & 0 & 0 & 0 \\ 16095 & 11233 & \mathbf{5 0 7 1} & \mathbf{9 2 6} & \mathbf{8 5 3} & \mathbf{2 5 0} & 0 & 0 & 0 & 0 & 0 & 35628 \\ 0 & 535 & 0 & 0 & 0 & 0 & 0 & 0 & 0 & 0 & 0 & 535 \\ 0 & 0 & 0 & 0 & 0 & 0 & 0 & 0 & 0 & 0 & 0 & 0 \\ 0 & 0 & 0 & 0 & 0 & 0 & 0 & 0 & 0 & 0 & 0 & 0 \\ 16095 & 11768 & \mathbf{5 0 7 1} & \mathbf{9 2 6} & \mathbf{8 5 3} & \mathbf{2 5 0} & 0 & 0 & 0 & 0 & 0 & 34963\end{array}$




\section{SPENT NUCLEAR FUEL PROJECT \\ WBS 1.4.1}

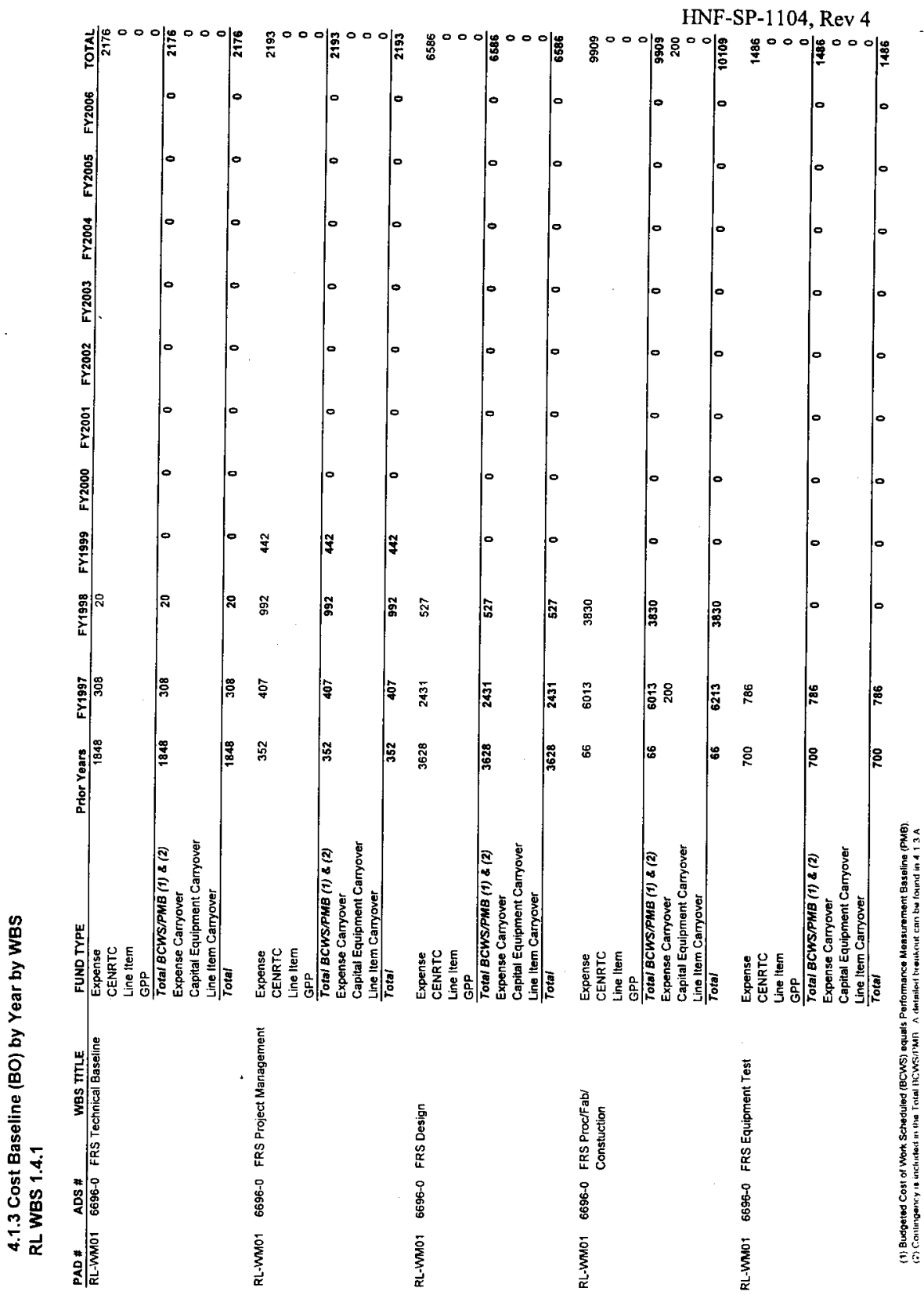


4.1.3 Cost Baseline (BO) by Year by WBS

RL WBS 1.4.1

RS Regulatory FUND TYPE FY1997 FY1998 FY1999 FY2000 FY2001 FY2002 FY2003 FY2004 FY2005 FY2006 TOTAL

Line Item

GPP

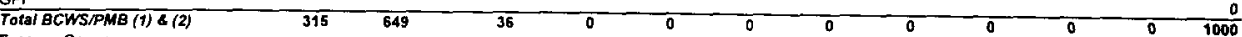

Expense Carryover

Capital Equipment Carryover

Line Item Carryover

Total

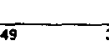

Sub-Total Fuel Removal

RL-WM01 6696-0 Sludge Removal System

Sub-Total Sludge Removal

$$
\begin{aligned}
& \text { Expense } \\
& \text { CENRTC } \\
& \text { Line Item } \\
& \text { GPP } \\
& \hline \text { Total BCW }
\end{aligned}
$$

(1) \& (2)

Capital Equipment Carryover

Line Item Carryover

Total

Expense

CENRTC

Line Item

Total BCWS-PMB (1) \& (2)

5405

Expense Carryover

Capital Equipment Carryover

Line Item Carryover

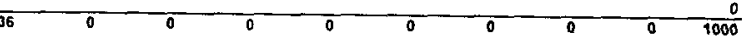

Tot

Expense
CENRTC
Line Item

Line It

Total BCWS/PMB (1) \& (2)

Expense Carryover

Capital Equipment Carryover

Line ltem Carryover

Total

Expense

CENRTC

Line Item

GPP

Torai BChSPMB (1)

Expense Cartyover

Caping Equipment Cerrover

Line liem Carryover

Total

\begin{tabular}{rr}
315 & \\
6909 & 1059 \\
0 & \\
0 & \\
0 & \\
\hline 6909 & 109 \\
0 & \\
0 & \\
0 & \\
\hline 6909 & 10794
\end{tabular}

\begin{tabular}{rrr}
36 & 0 & 0 \\
5405 & 442 & 0 \\
0 & 0 & 0 \\
0 & 0 & 0 \\
0 & 0 & 0 \\
5405 & 442 & 0 \\
0 & 0 & 0 \\
0 & 0 & 0 \\
0 & 0 & 0 \\
\hline
\end{tabular}

\begin{tabular}{ll}
0 & 0 \\
0 & 0 \\
0 & 0 \\
0 & 0 \\
\hline 0 & 0 \\
0 & 0 \\
0 & 0 \\
0 & 0 \\
\hline
\end{tabular}

0
0
0
0
0
0
0
0

\begin{tabular}{rlllr}
0 & 0 & 0 & 0 & 23350 \\
0 & 0 & 0 & 0 & 0 \\
0 & 0 & 0 & 0 & 0 \\
0 & 0 & 0 & 0 & 0 \\
\hline 0 & 0 & 0 & 0 & 23350 \\
0 & 0 & 0 & 0 & 200 \\
0 & 0 & 0 & 0 & 0 \\
0 & 0 & 0 & 0 & 0 \\
\hline 0 & 0 & 0 & 0 & 23550
\end{tabular}

$\begin{array}{lllll}2966 & 2229 & 5561 & 6117 & 693\end{array}$

17566

0

RL-WM01 6696-0 Debris Removal Project

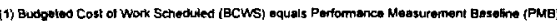

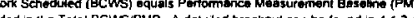


HNF-SP-1 104, Rev 4

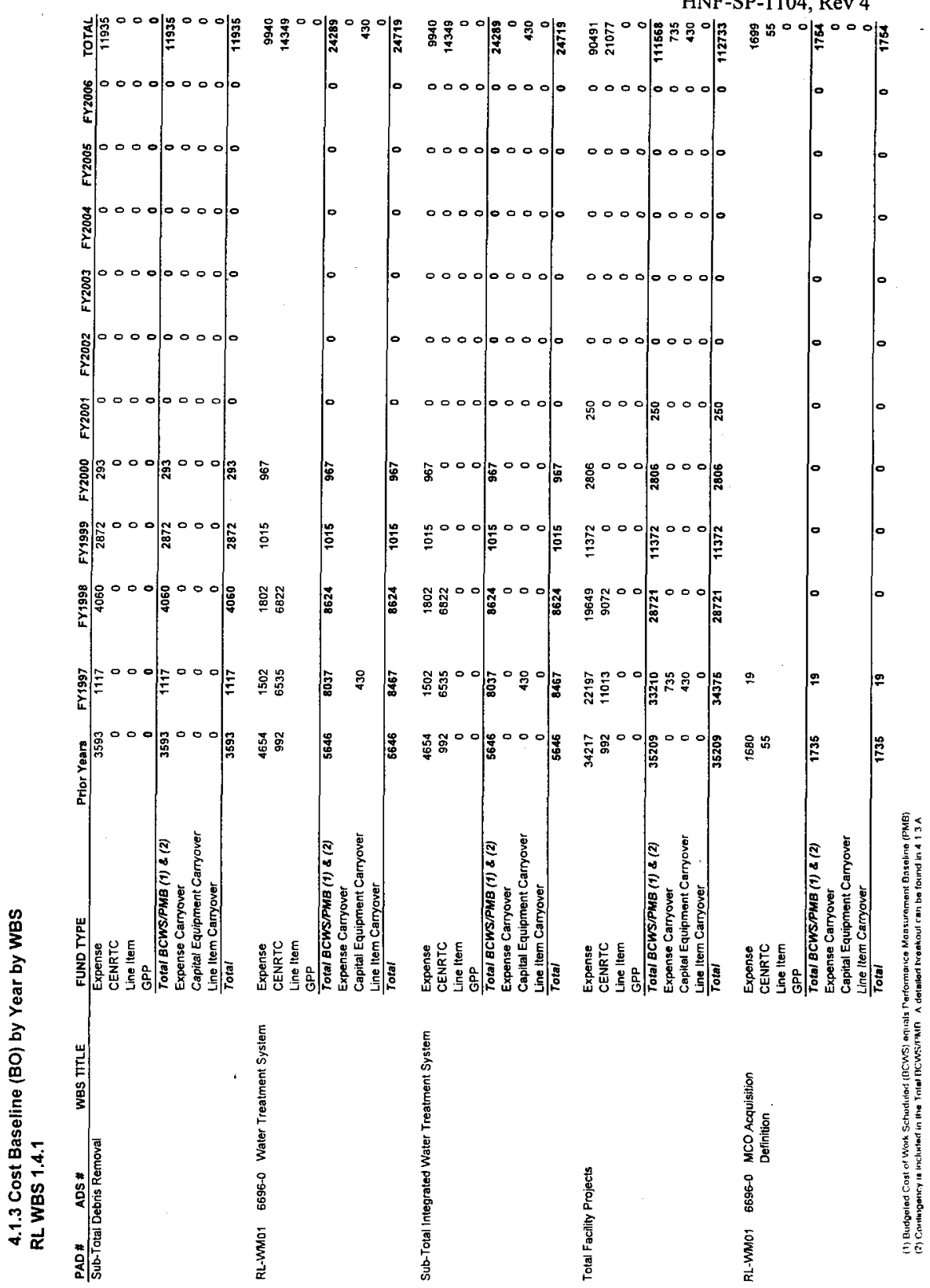


4.1.3 Cost Baseline (BO) by Year by WBS

RL WBS 1.4.1

\begin{tabular}{|c|c|c|c|c|c|c|c|c|c|c|c|c|c|c|c|}
\hline PAD \# & ADS \# & WBS TITLE & FUND TYPE & Prior Years & FY1997 & FY1998 & FY1999 & FY2000 & EY2001 & FY2002 & FY2003 & FYzo04 & FY2005 & FY2006 & TOTAL \\
\hline \multirow[t]{3}{*}{ RL-WMO1 } & $6696-0$ & $\begin{array}{l}\text { MCO Project } \\
\text { Management }\end{array}$ & $\begin{array}{l}\text { Expense } \\
\text { CENRTC } \\
\text { Line trem } \\
\text { GPP } \\
\end{array}$ & 720 & 810 & 748 & 274 & 226 & & & & & & & $\begin{array}{r}2778 \\
0 \\
0 \\
0 \\
\end{array}$ \\
\hline & & & $\begin{array}{l}\text { Total BCWSPME (1) \& (2) } \\
\text { Expense Carryover } \\
\text { Capital Equipment Carryover } \\
\text { Line Item Carryover }\end{array}$ & 720 & $\overline{810}$ & $\overline{748}$ & 274 & 226 & $\overline{0}$ & 0 & $\overline{0}$ & 0 & 0 & 0 & $\begin{array}{r}2778 \\
0 \\
0 \\
0 \\
\end{array}$ \\
\hline & & & Total & 720 & 840 & $74 \overline{8}$ & $27 \overline{4}$ & 226 & 0 & 0 & $\overline{0}$ & 0 & 0 & 0 & 2778 \\
\hline \multirow[t]{3}{*}{ RL-WMOT } & $6696-0$ & MCO Design & $\begin{array}{l}\text { Expense } \\
\text { CENRTC } \\
\text { Line ltem } \\
\text { GPP }\end{array}$ & 1170 & 948 & & & & & & & & & & $\begin{array}{r}2118 \\
0 \\
0 \\
0 \\
\end{array}$ \\
\hline & & & $\begin{array}{l}\text { Total BCWS/PMB (1) \& (2) } \\
\text { Expense Carryover } \\
\text { Capital Equipment Carryover } \\
\text { Line ltem Cartyover }\end{array}$ & 1170 & 948 & 0 & 0 & 0 & 0 & 0 & 0 & 0 & 0 & 0 & $\begin{array}{r}2118 \\
0 \\
0 \\
0 \\
\end{array}$ \\
\hline & & & Total & 1170 & $\overline{948}$ & 0 & $\overline{0}$ & 0 & 0 & $\overline{0}$ & $\overline{0}$ & 0 & 0 & 0 & 2918 \\
\hline \multirow[t]{3}{*}{ RL-WMO1 } & $6696-0$ & MCO Fabrication & $\begin{array}{l}\text { Expense } \\
\text { CENRTC } \\
\text { Line Item } \\
\text { GPP. }\end{array}$ & & 1746 & 10774 & 17926 & 6694 & & & & & & & $\begin{array}{r}37137 \\
0 \\
0 \\
0 \\
\end{array}$ \\
\hline & & & $\begin{array}{l}\text { Total BCWS } / P M B(1) \&(\overline{2}) \\
\text { Expense Carryover } \\
\text { Capital Equipment Carryover } \\
\text { Line Item Carryover }\end{array}$ & $\overline{0}$ & 1746 & 10771 & 17926 & 6694 & 0 & $\overline{0}$ & $\overline{0}$ & 0 & 0 & 0 & $\begin{array}{r}37137 \\
0 \\
0 \\
0 \\
\end{array}$ \\
\hline & & & Total & 0 & 1746 & 10779 & 17926 & 6694 & 0 & 0 & 0 & 0 & $\overline{0}$ & $\overline{0}$ & $\overline{37137}$ \\
\hline \multirow[t]{3}{*}{ RL-WM01 } & $6696-0$ & $\begin{array}{l}\text { Testing \& Qualification } \\
\text { of MCO's }\end{array}$ & $\begin{array}{l}\text { Expense } \\
\text { CENRTC } \\
\text { Line Item } \\
\text { GPP }\end{array}$ & 1078 & 147 & : & & & & & & & & & $\begin{array}{r}1225 \\
0 \\
0 \\
0 \\
\end{array}$ \\
\hline & & & $\begin{array}{l}\text { Total BCWS/PMB (1) \& (2) } \\
\text { Expense Carryover } \\
\text { Capital Equipment Carryover } \\
\text { Line lem Carryover }\end{array}$ & 1078 & 147 & $\overline{0}$ & $\overline{0}$ & 0 & 0 & 0 & 0 & 0 & $\overline{0}$ & 0 & $\begin{array}{r}\overline{1225} \\
0 \\
0 \\
0 \\
\end{array}$ \\
\hline & & & Total & 1078 & 147 & 0 & $\overline{0}$ & 0 & 0 & 0 & 0 & $\overline{0}$ & 0 & 0 & $\overline{1225}$ \\
\hline \multirow[t]{3}{*}{ RL-WMO1 } & $6696-0$ & $\begin{array}{l}\text { MCO Topical Safely } \\
\text { Report }\end{array}$ & $\begin{array}{l}\text { Expense } \\
\text { CENRTC } \\
\text { Line Item } \\
\text { GPP }\end{array}$ & 225 & 256 & 132 & & & & & & & & & $\begin{array}{r}613 \\
0 \\
0 \\
0 \\
\end{array}$ \\
\hline & & & $\begin{array}{l}\text { Totai BCWS/PMB (1) \&(2) } \\
\text { Expense Carryover } \\
\text { Capital Equipment Carryover } \\
\text { Line Item Carryover }\end{array}$ & 225 & 256 & 132 & $\overline{0}$ & 0 & $\overline{0}$ & $\overline{0}$ & $\overline{0}$ & - & 0 & 0 & $\begin{array}{r}6 \overline{13} \\
0 \\
0 \\
0 \\
\end{array}$ \\
\hline & & & Total & 225 & 256 & 132 & 0 & 0 & 0 & 0 & 0 & 0 & 0 & $\overline{0}$ & 613 \\
\hline
\end{tabular}

(1) Budgetiod Cost of Work Scheduled (BCWS) mquals Pertomanice Motsurement Baseline (PMB)

(2) Comtingency is inchured in the Total BCWS/PMB A detailed breakout ean be found in 4.1.3.A 


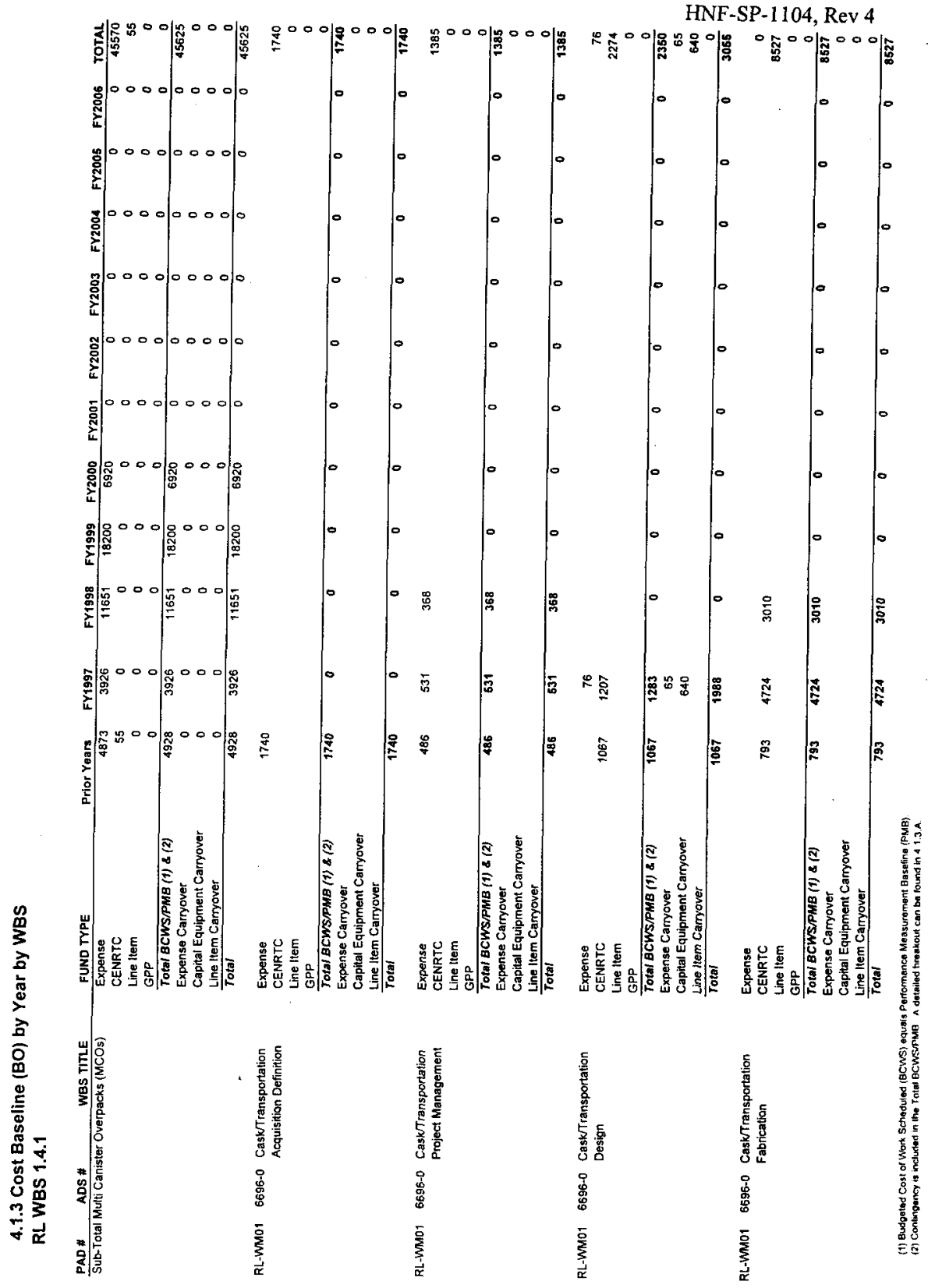




\section{SPENT NUCLEAR FUEL PROJECT}

WBS 1.4.1

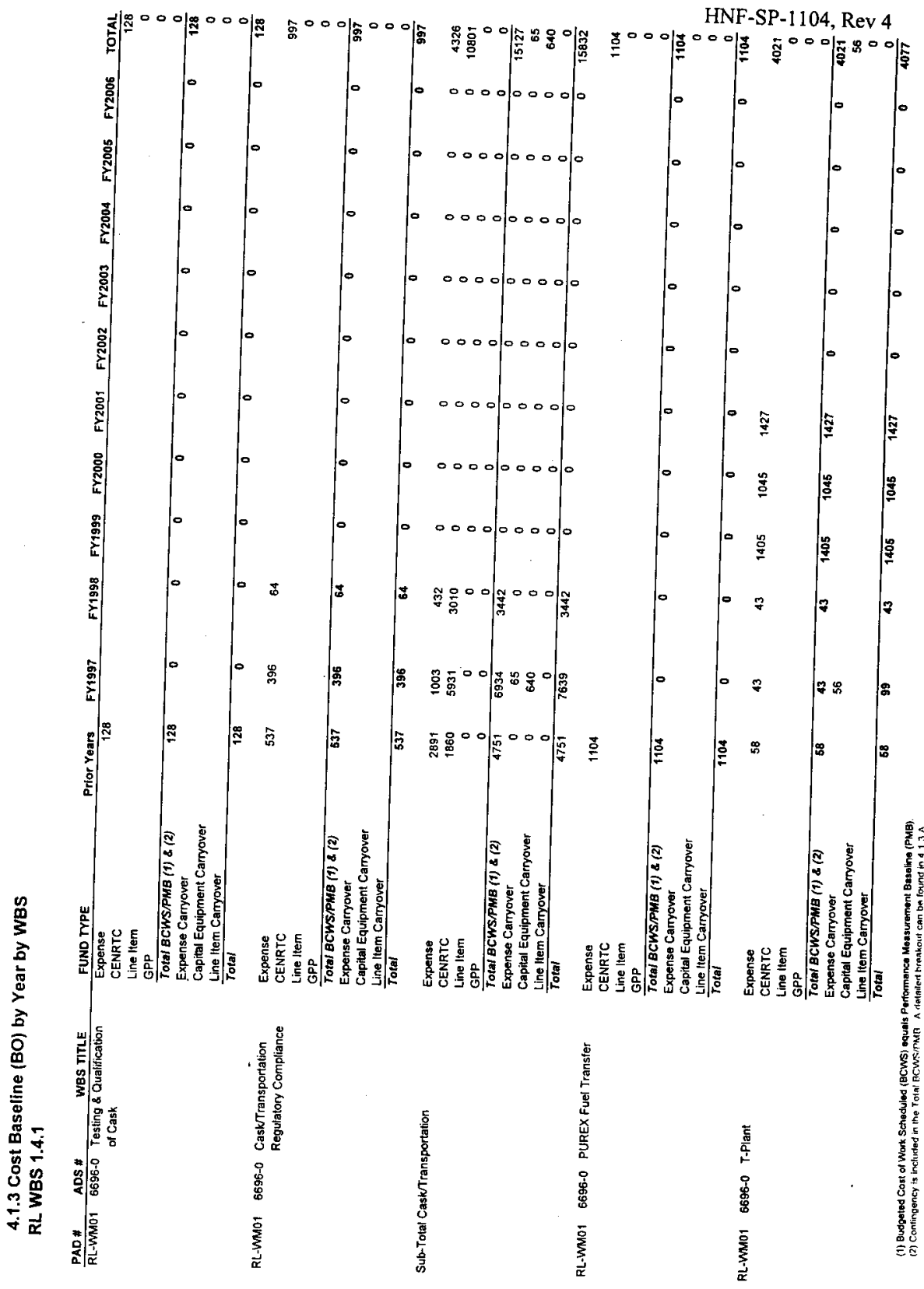


4.1.3 Cost Baseline (BO) by Year by WBS

RL WBS 1.4.1

PAD \# ADS \# WBS TITLE

FUND TYPE Expense

Y1997 FY1998 FY1999 FY2000 FY2001 FY2002 FY2003 FY2004 FY2005 FY2006 TOTAL CENRTO

Line ltem

GPP

Total BCWS/PMB (1) \& (2)

$11 \quad 11$

Expense Carryover

Capital Equipment Carryover

Line Item Carryover

Total

RL-WMO1 6696-0 PNL Labs, Bldgs, 324 325,327

\section{Expense}

CENRTC

Line Item

GPP

Totai BCWSTPB (1) \& (2) ___

Expense Carryover

17

Capital Equipment Carryover

Line item Carryover

Total

RL-WMO1 6696-0 308 Annex

\section{Expense}

CENRTC

Line Item

Line

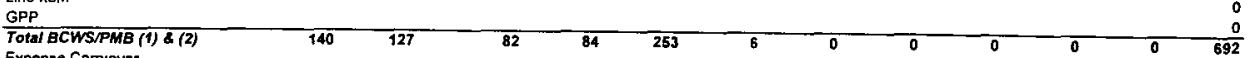

Expense Caryover

Capital Equipment Carryover

Line Item Carryover

$$
\text { Total }
$$

$$
237
$$$$
103
$$$$
248-32
$$

\section{Expense}

CENRTC

Line Item

Line

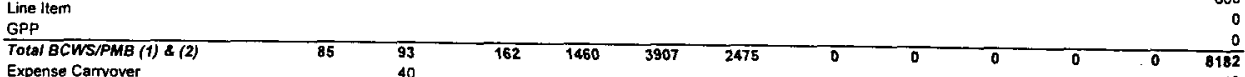

Expense Cartyover

Capital Equipment Carryover

Line ltem Carryover

Total

Expense

CENRTC

Line Item

GPP

Total BCWS Pito (1)

Expense Carryover

Capital Equipment Carryover

Line Item Carryover

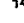

27

82

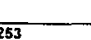

Total

85

93

1085

$3582 \quad 2475$

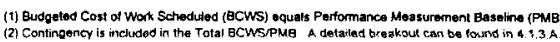

(2) Contingency is induded in the Total BCWSPMM A detaled breaksut con be fourts in $4.1 .3 \mathrm{~A}$ 
4.1.3 Cost Baseline (BO) by Year by WBS

\section{RL WBS 1.4.1}

PAD \# ADS \# WES TITLE

FUND TYPE

Prior Years FY1997

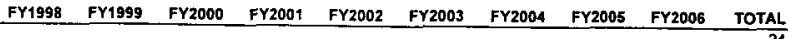
Expense CENRTC Line Item GPP

Total BCWS/PMB (1) $\overline{\mathrm{s}(2)}$

Expense Carryover

Capital Equipment Carryover

Line Item Carryover

Total

RL-WMO1 6696-0 National Programs

Expense

CENRTC

Line Item

GPP

Total BCWS/PMA (1) \& (2)

Toral BCWSismb (1)

Expense Carryover

Capital Equipment Carryover

Line liem Carryover

Total

$\frac{\text { Years FY1997 }}{24}$

\pm Sub-Total Other Hanford Fuel

Expense

CENRTC

Line liem

GPP

Total BCWS/PMB (1) \& (2)

Expense Carryover

Capital Equipment Carryover

Line hem Carryover

Total

Total SNF Storage

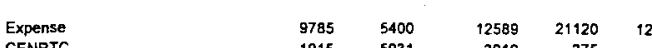

\begin{tabular}{|c|c|c|c|c|c|c|c|c|c|c|}
\hline Expense & 9785 & 5400 & 12589 & 21120 & 12394 & 3970 & 0 & 0 & 0 & 65258 \\
\hline
\end{tabular}

Line ltem

GPP

Total BCWS/PMB (1) \& (2)

Expense Carryover

Capital Equipment Carryover

Line Item Carryover

Total

$\begin{array}{rrr}1915 & 5931 & 3010 \\ 0 & 0 & \\ 0 & 0 & 0\end{array}$

$\begin{array}{rrr}0 & 375 & 225 \\ 0 & 0 & 0\end{array}$

$11700-11331$

0 o

0

$\begin{array}{rrrr}15599 & 21495 & 12619 & 3970\end{array}$

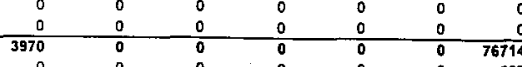

0

640
0

00

RL-WM01 6696-1 CSB Project

Manageme

Expense

CENRTC

Line tem

$5436+121 \quad 80$

$21495-12619$

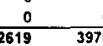

0
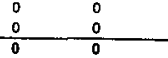

Z

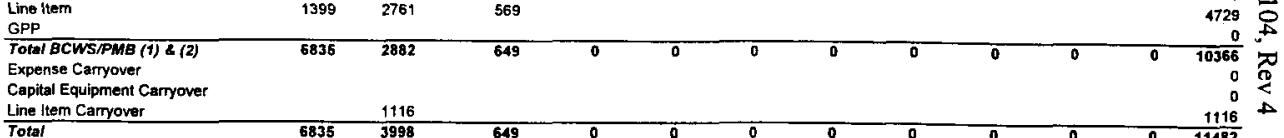

Line Item Carryover

649

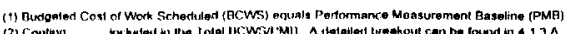




\section{SPENT NUCLEAR FUEL PROJECT}

WBS 1.4.1

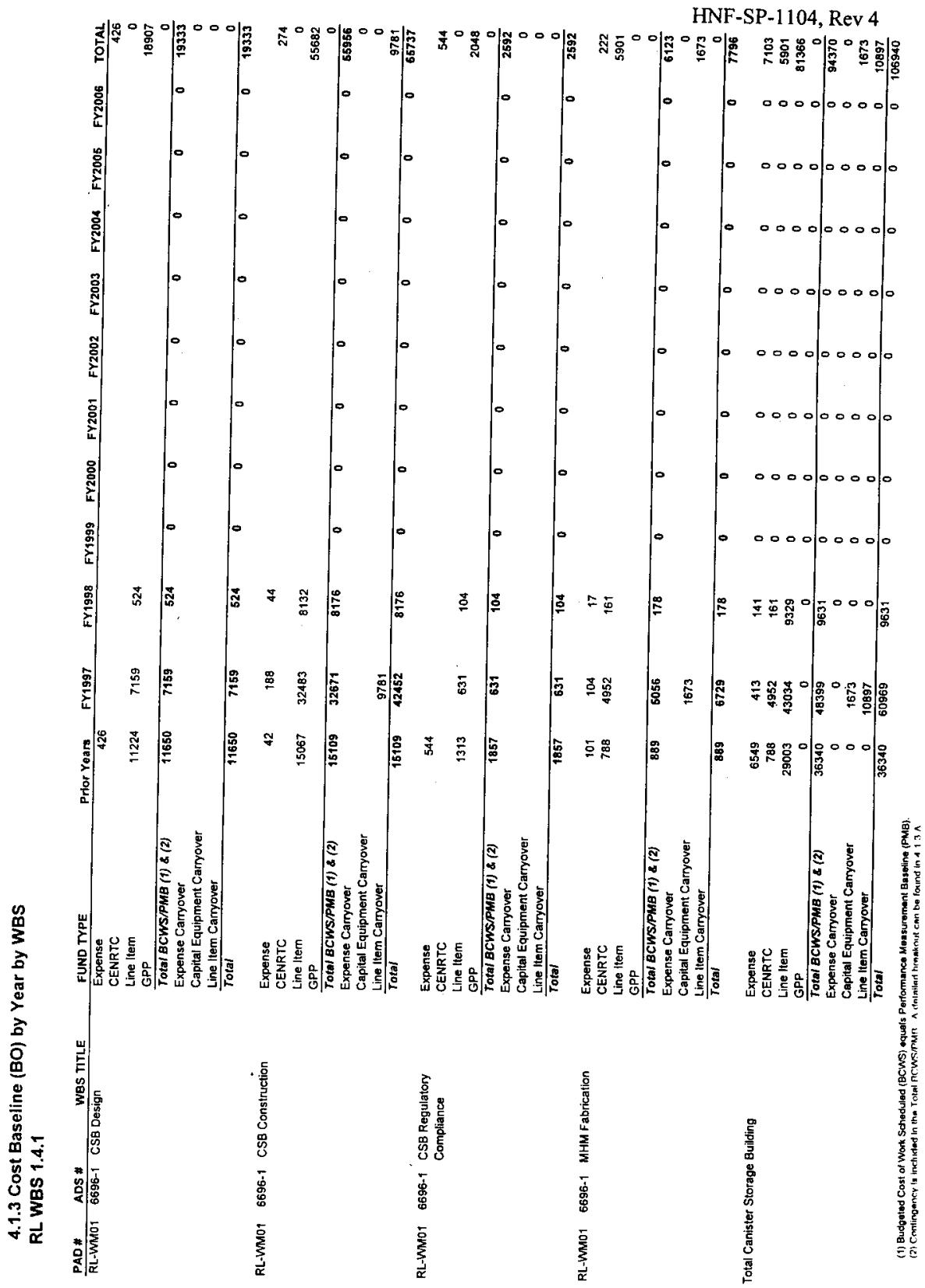


4.1.3 Cost Baseline (BO) by Year by WBS

\section{RL WBS 1.4.1}

PAD : ADS* WES TITLE Expense Prior Years FY1997 FY1998 FY1999 CENRTC

Line ltem

GPP

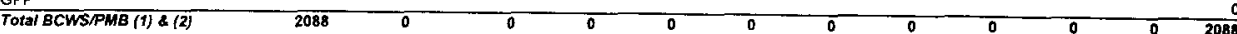

Expense Cartyover

Capital Equipment Carryover

Line liem Carryover

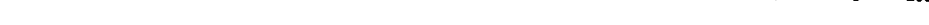

Total

2088

RL-WM01 6696-1 CVD Project Management

\section{Expense}

CENRTC

Line Item

GPP

Total BCWS/PMB (1) \& (2)

Expense Carryover

Capital Equipment Carryover

Line Item Carryover

Total

213

$102 \quad 970$

$970 \quad 110$

315970

$970-116$

0

0

RL.WM01 6696-1 CVD Design

Total

$315 \quad 97$

Expense

CENRTC
Line Item 368

Line Item

$1422 \quad 650$

Total BCWSTPB (1) \& (2)

1790

50

Expense Carryover

Capital Equipment Carryover

Line Item Carryover

Line Item

4790

\section{Expense}

CENRTC

Line item

GPP

$162 \quad 8168 \quad 1300$

Total BCWS/PMB (1) \& (2)

$162 \quad 8168$

Expense Carryover

Capital Equipment Carryover

Line Item Carryover

300

rotsi

162

$168-7$

\section{Expense}

CENRTC

Line Item

GPP

Total BCWS/PMB (1) \& (2)

Expense Carryover

Capital Equipment Carryover

Line Item Carryover

$466 \quad \quad 327$

$46 \overline{3} \quad 327$

327

$$
\text { Total }
$$

466

327

1300 


\subsubsection{Cost Baseline (BO) by Year by WBS}

\section{RL WBS 1.4.1}

PAD \# ADS \# Sub-Total Cold Vacuum Drying

WBS TTLE

FUND TYPE

\begin{tabular}{rrrrrrrrrrrr} 
Prior Years & FY1997 & FY1998 & FY1999 & FY2000 & FY2001 & FY2002 & FY2003 & FY2004 & FY2005 & FY2006 & TOTAL \\
\hline 2669 & & & 0 & 0 & & & & & &
\end{tabular}

CENRTC

GPP

Total BCWS/PMB (1) \& (2)

Expense Carryover

Capital Equipment Carryover

Line Item Carryover

Total

\begin{tabular}{rrrll}
2669 & 0 & 0 & 0 & 0 \\
0 & 0 & 0 & 0 & 0 \\
2152 & 10115 & 1416 & 0 & 0 \\
0 & 0 & 0 & 0 & 0 \\
\hline 4821 & 10115 & 1416 & 0 & 0 \\
0 & 0 & 0 & 0 & 0 \\
0 & 0 & 0 & 0 & 0 \\
0 & 0 & 0 & 0 & 0 \\
4821 & 10115 & 1416 & 0 & 0
\end{tabular}

\begin{tabular}{rrrrrrr}
0 & 0 & 0 & 0 & 0 & 0 & 2669 \\
0 & 0 & 0 & 0 & 0 & 0 & 0 \\
0 & 0 & 0 & 0 & 0 & 0 & 13683 \\
0 & 0 & 0 & 0 & 0 & 0 & 0 \\
\hline 0 & 0 & 0 & 0 & 0 & 0 & 16352 \\
0 & 0 & 0 & 0 & 0 & 0 & 0 \\
0 & 0 & 0 & 0 & 0 & 0 & 0 \\
0 & 0 & 0 & 0 & 0 & 0 & 0 \\
0 & 0 & 0 & 0 & 0 & 0 & 16352
\end{tabular}

RL-WM01 6696-1 HCS Acquistition

Expense

$1043 \quad 201$

Definition

CENRTC

Line item

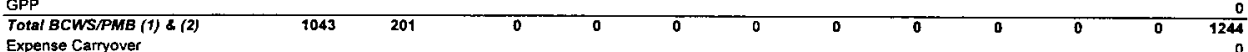

Capital Equipment Carryover

Line ltem Carryover

Total

Expense

CENRTC

Line Item
GPP

Total BCWSiPMB (1) \& (2)

Expense Carryover

Capital Equipment Carryover

Line Item Carryover

Total

Expense

CENRTC

Lina Item

Lino It

Total BCWSPMB (f) \& (2)

Expense Carryover

Capital Equipment Carryover

Line Item Carryover

Total

124

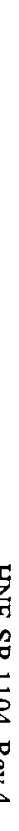

RL.WMO1 6696-1 HCS Construction

Expense

CENRTC

Line Item

Line

$4515-1242$

Total BCWS/PMB (1) \& (2)

Tola BCWSTMB

Capital Equipment Carryover

Line Item Carryover

$$
\text { Total }
$$

0

4515

1242

0

(1) Burgeter Cost of Woit Schedular (ACWSi equals Pertormance Measurement Baseltne (PMM)

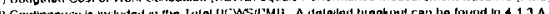




\section{SPENT NUCLEAR FUEL PROJECT WBS 1.4.1}

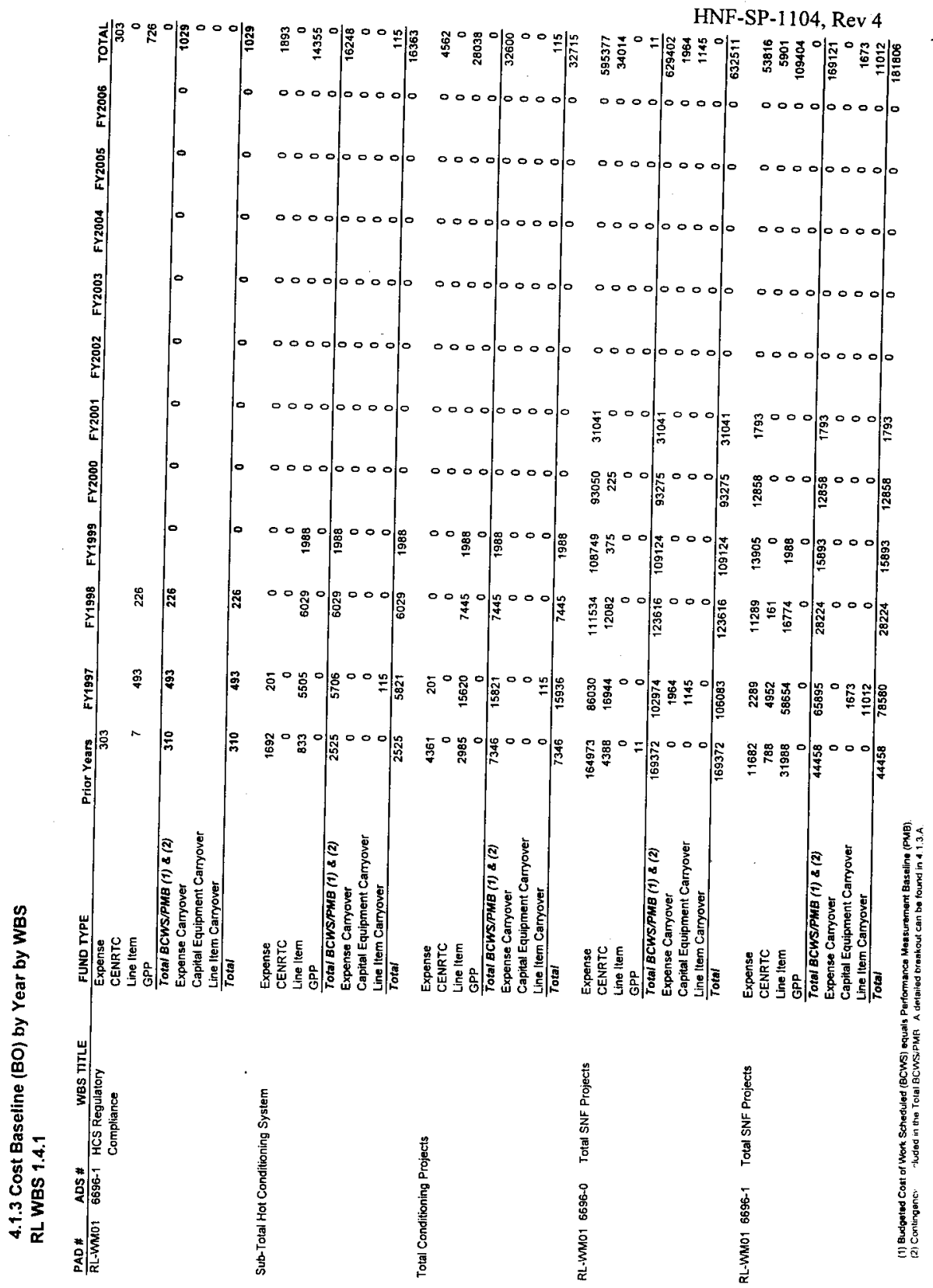


4.1.3 Cost Baseline (BO) by Year by WBS

RL WBS 1.4.1

PAD \# ADS \# WBS TITLE

FUND TYPE

Expense

CENRTC Prior Years FY1997

Line ltem

GPP

319885865

Total BCWS/PMB (1) \& (2) $\quad 213830 \quad 16886$

Expense Carryover

Capital Equipment Carryover

$\begin{array}{rr}113830 & 168869 \\ 0 & 1964\end{array}$

Line Item Carryover

\begin{tabular}{rr}
0 & $110 \uparrow 2$ \\
\hline
\end{tabular}

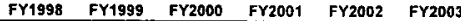

\begin{tabular}{rrrrr}
3 & FY2004 & FY2005 & FY2006 & TOTAL \\
0 & 0 & 0 & 0 & $\mathbf{6 4 9 1 9 3}$ \\
0 & 0 & 0 & 0 & 39915 \\
0 & 0 & 0 & 0 & 109404 \\
0 & 0 & 0 & 0 & 11 \\
\hline 0 & 0 & 0 & 0 & $\mathbf{7 9 8 5 2 3}$ \\
0 & 0 & 0 & 0 & 1964 \\
0 & 0 & 0 & 0 & 2818 \\
0 & 0 & 0 & 0 & 11012 \\
0 & 0 & 0 & 0 & 814317
\end{tabular}

$\begin{array}{rrr}122823 & 122654 & 105 \\ 12243 & 375 & 225\end{array}$

$12243 \quad 375 \quad 225 \quad 328$

$\begin{array}{rrr}0 & 0 & 0 \\ 151840 & 125017 & 105133\end{array}$

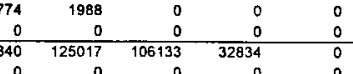

$\begin{array}{rrrrrrrrr}0 & 0 & 0 & 0 & 0 & 0 & 0 & 0 & 0 \\ 11012\end{array}$


4.1.3.A Contingency by Year by WBS

RL WBS 1.4.1

\begin{tabular}{|c|c|c|c|c|c|c|c|c|c|c|c|c|c|c|}
\hline \multirow[t]{2}{*}{ RL-WMO1 } & $\frac{A D S *}{6696-0}$ & $\frac{\text { WBS TITLE }}{\text { Project Direction }}$ & $\begin{array}{l}\text { FUND TYPE } \\
\text { Expense } \\
\text { CENRTC } \\
\text { Line Item } \\
\text { GPP }\end{array}$ & FY1997 & $\begin{array}{r}\text { FY1998 } \\
4854\end{array}$ & $\frac{\text { FY1999 }}{11000}$ & $\begin{array}{r}\text { FY2000 } \\
10000\end{array}$ & $\frac{F Y 2001}{4500}$ & FY2002 & $\mathrm{FY} 2003$ & FY2004 & FY2005 & FY2006 & $\begin{array}{r}\text { TOTAL } \\
30354 \\
0 \\
0\end{array}$ \\
\hline & & & Total Contingency & 0 & 4854 & 11000 & 10000 & 4500 & $\overline{0}$ & 0 & 0 & 0 & 0 & 30354 \\
\hline \multirow[t]{2}{*}{ RL-WMO1 } & $6696-0$ & $\begin{array}{l}\text { FRS Procifab/ } \\
\text { Construction }\end{array}$ & $\begin{array}{l}\text { Expense } \\
\text { CENRTC } \\
\text { Line Hem } \\
\text { GPP }\end{array}$ & 497 & 603 & & & & & & & & & $\begin{array}{r}1100 \\
0 \\
0\end{array}$ \\
\hline & & & Total Contingency & 497 & 603 & 0 & 0 & 0 & 0 & o & 0 & $\overline{0}$ & 0 & 1100 \\
\hline \multirow{2}{*}{\multicolumn{2}{|c|}{ Total Facility Projects }} & & $\begin{array}{l}\text { Expense } \\
\text { CENRTC } \\
\text { Line Item } \\
\text { GPP }\end{array}$ & 497 & 603 & 0 & 0 & 0 & 0 & 0 & 0 & 0 & 0 & $\begin{array}{r}1100 \\
0 \\
0 \\
0\end{array}$ \\
\hline & & & Total Contingency & 497 & 603 & 0 & 0 & 0 & 0 & $\overline{0}$ & 0 & 0 & 0 & 1100 \\
\hline RL-WM01 & $6696-1$ & CSB Project Managernent & $\begin{array}{l}\text { Expense } \\
\text { CENRTC } \\
\text { Line ftem } \\
\text { GPP }\end{array}$ & 1992 & & & & & & & & & & $\begin{array}{r}0 \\
0 \\
1992 \\
0\end{array}$ \\
\hline \multirow[t]{2}{*}{ RL-WM01 } & $6696-1$ & MHM Fabrication & $\begin{array}{l}\text { Expense } \\
\text { CENRTC } \\
\text { Line Item } \\
\text { GPP } \\
\end{array}$ & 200 & & & & & & & & . & & $\begin{array}{r}0 \\
200 \\
0 \\
0\end{array}$ \\
\hline & & & Total Contingency & 200 & $\overline{0}$ & 0 & $\overline{0}$ & 0 & $\overline{0}$ & 0 & 0 & 0 & 0 & 200 \\
\hline \multirow[t]{4}{*}{ Total Canis } & ster Storage & e Building & Expense & 0 & 0 & 0 & 0 & 0 & 0 & 0 & 0 & 0 & 0 & 0 \\
\hline & & & CENRTC & 200 & 0 & 0 & 0 & 0 & 0 & 0 & 0 & 0 & 0 & 200 \\
\hline & & & Line ltem & 1992 & 0 & 0 & 0 & 0 & 0 & 0 & 0 & 0 & 0 & 1992 \\
\hline & & & Total Contingency & $\frac{0}{2192}$ & $\frac{0}{0}$ & $\frac{0}{0}$ & $\frac{0}{0}$ & $\frac{0}{0}$ & $\frac{0}{0}$ & $\frac{0}{0}$ & $\frac{0}{0}$ & $\frac{0}{0}$ & $\frac{0}{0}$ & $\frac{0}{2192}$ \\
\hline RL-MM01 & $6696-1$ & $\begin{array}{l}\text { Cold Vacuum Drying } \\
\text { Construction }\end{array}$ & $\begin{array}{l}\text { Expense } \\
\text { CENRTC } \\
\text { Line hem } \\
\text { GPP } \\
\text { Total Contingency }\end{array}$ & 500 & $\overline{0}$ & 0 & 0 & & 0 & 0 & 0 & & & $\begin{array}{r}0 \\
0 \\
500 \\
0 \\
500\end{array}$ \\
\hline
\end{tabular}




\section{SPENT NUCLEAR FUEL PROJECT WBS 1.4.1}

HNF-SP-1104, Rev 4

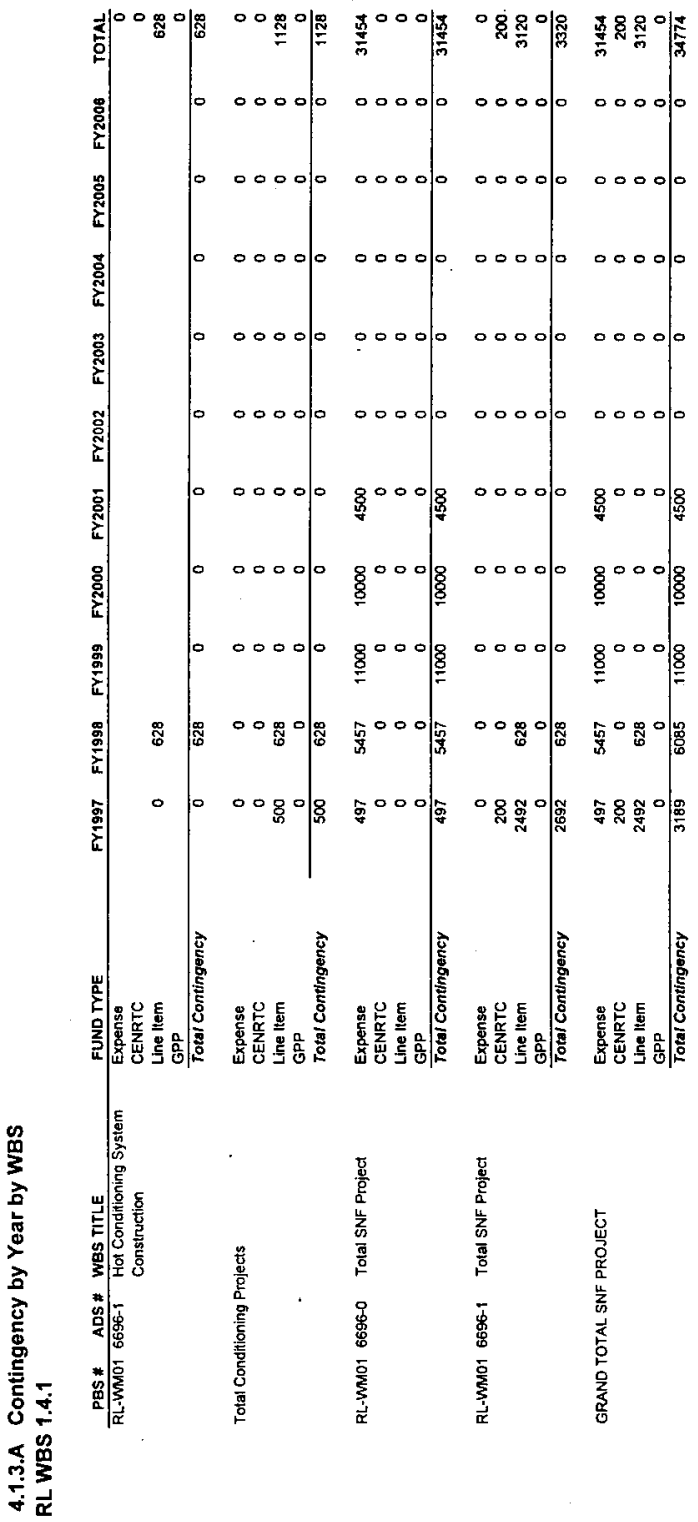


This page intentionally left blank. 


\section{SPENT NUCLEAR FUEL PROJECT WBS 1.4.1}

HNF-SP-1104, Rev 4

This page reserved for Exhibit 4.1.4 Cost Baseline Execution Year by Month.

4.1.4 
This page intentionally left blank. 


\section{SPENT NUCLEAR FUEL PROJECT \\ WBS 1.4.1}

HNF-SP-1104, Rev 4

\subsection{Basis of Estimate}

The cost estimates for the Spent Nuclear Fuel (SNF) project have been prepared to provide a cost profile of the remaining work scope required to remove 2100 Metric Tons of spent nuclear fuel from the K-Basins to dry interim storage in the Canister Storage Building, management of Other Hanford SNF, and planning for transition of the K-Basin facilities to Transition Projects. Estimate to Complete (ETC) costs have been reviewed by individual Sub-Projects (listed below) and assessed for reasonableness. Estimates are identified as having used analogy, trend analysis, expert opinion, parametric methodology, or comparable historical activities as their basis. Cost estimates have been developed and maintained throughout the life of each Sub-Project.

With the scope of the SNF Project activities defined, cost estimates and schedules have been developed. The complexity of developing these estimates for a resource loaded schedule required the involvement of many disciplines covering the entire spectrum of planning, scheduling, and estimating. The disciplines that provided input included technical, operational, project management, maintenance, engineering, safety, health physics, quality assurance, and security.

The estimates used current Company direct-labor rates escalated using RL-approved escalation rates. The overhead factors are shown below:

General \& Administration (G\&A) \& PHMC Fee

$16.7 \%$

Material Procurement Rate (MPR)

$7.0 \%$

The following estimate packages are located with the Planning \& Schedule Integration Group at 2751/200E:

Safety \& Quality

Project Integration

Operations

Facility Upgrades

Fuel Retrieval

Sludge Retrieval

Debris Removal

Integrated Water Treatment

Multi-Canister Overpack

Cask/Transportation

Other Hanford Fuel

Canister Storage Building

Hot Conditioning System

Cold Vacuum Drying System 
This page intentionally left blank. 
Exhibit: Program Average FTE Projections by cocs Categories

Project Hanford Breakdown Structure/Title: 1.4.1 Spent Nuclear Fuel Project

\begin{tabular}{|c|c|c|c|c|c|c|}
\hline COCS & Title & FY 1997 & FY 1998 & FY 1999 & FY 2000 & FY 2001 \\
\hline $\mathrm{COOO}$ & Crafts & 78 & 78 & 80 & 80 & 35 \\
\hline E000 & Engineers & 190 & 165 & 155 & 105 & 50 \\
\hline G000 & General Admin, Secretarial \& Clerical Support & 54 & 52 & 47 & 42 & 25 \\
\hline Loo0 & Laborers and General Service Workers & 9 & 11 & 13 & 12 & 8 \\
\hline Moo0 & Gen Mgrs, Exec, 1st Line Suprv'sr \& Prog/Proj Mgrs & 70 & 67 & 63 & 50 & 20 \\
\hline POOO & Professional Administrative \& Related Occupations & 47 & 45 & 42 & 38 & 20 \\
\hline Roo0 & Operators & 60 & 100 & 120 & 120 & 54 \\
\hline Sooo & Scientists & 1 & 1 & 1 & & \\
\hline T000 & Technicians & 52 & 71 & 73 & 70 & 22 \\
\hline Total & & 561 & 590 & 594 & 517 & 234 \\
\hline
\end{tabular}

NOTE: Includes DESH, major subcontractors, and ENCO personnel. 
HNF-SP-1104 Rev 4

Exhibit: Program Average FTE Projections by COCS Categories

Includes Major Subcontractor and EntCo FTEs Project Haniord Breakdown Structure/Title: Spent Nuclear Fuel Project

\begin{tabular}{|c|c|c|c|c|c|c|c|c|c|c|c|}
\hline COCS & Title & FY 1997 & FY 1998 & FY 1999 & FY 2000 & FY 2001 & FY 2002 & FY 2003 & FY 2004 & FY 2005 & FY 2006 \\
\hline $\mathrm{CO00}$ & Crafts & 78 & 78 & 801 & 80 & 35 & & & & & \\
\hline $\mathrm{co10}$ & Carpenters & 4 & 4 & & . & & & & & & \\
\hline $\mathrm{CO} 20$ & Electricians & 17 & 17 & & & & & & & & \\
\hline $\cos 0$ & Heating Air-Conditioning and Refrig Mechanics (HVAC) & & & & & & & & & & \\
\hline $\mathrm{CO40}$ & Machinists & & i & & & & & 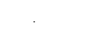 & & & \\
\hline $\cos 0$ & Masons & 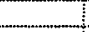 & & & & & & & & & \\
\hline $\mathrm{CO60}$ & Milwrighls... & 19 & 19 & & & & & , & & & $\cdot$ \\
\hline $\mathrm{Co70}$ & Painters & 6 & 6 & & & & & & & & \\
\hline COBO & Plumbers and Pipefitters & 16 & 16 & . & & & & & & & \\
\hline c090 & Structural and Metal Workers & 2 & 2 & & & & & & & & \\
\hline $\mathrm{C} 100$ & Vehicle and Mobile Equipment Mechanics & & & • & & & & & & & \\
\hline C110 & Welders & & & & & & & & & & \\
\hline $\mathrm{C} 120$ & Other Crafts & 14 & 14 & & & & & & & & \\
\hline E000 & Engineers & 190 & 165 & 155 & 105 & 50 & & & & & \\
\hline E010 & Chemical Engineers & 23 & 15 & & & & & & & & \\
\hline $\mathrm{EO} 20$ & Civil Engineers ... & 5 & 5 & & . & & & & & & \\
\hline E040 & Electrical Engineers & 7 & 7 & & & & & & & & \\
\hline E050 & Environmental Engineers & 3 & 3 & & & & & & & & \\
\hline E060 & industrial Engineers & & & & & & & & & & \\
\hline E்070 & Mechanical Engineers & 34 & 34 & & & & & & . & & \\
\hline E080 & Nuclear Engineers & 1 & 1 & & & & & & & & \\
\hline E090 & Petroleum/Mining Engineers & & & & . & & & & & & \\
\hline E100 & Plant Engincers & 53 & 45 & & & & & & & & $\therefore$ \\
\hline E110 & Quality Assurance/Control Engineers & 5 & 5 & & $\cdot$ & & & $=$ & & & $\because$ \\
\hline E120 & Safely Engineers & 6 & 6 & & . & & & & & & \\
\hline E130 & Other Engineers & 51 & 42 & & & & & & & & \\
\hline E140 & Construclion Engineers & 2 & 2 & & & & & ' & & & \\
\hline Go00 & General Admin, Secretarial \& Clerical Support & 54 & 52 & 47 & 42 & 25 & & & & & \\
\hline G010 & Administrative Assistants & 8 & 7 & & & & & & & & \\
\hline G020 & Office Clerks (General) & 17 & 16 & & & & & & & & \\
\hline G030 & Office Clerks (Specialized) & 7 & 7 & & & & & & & & \\
\hline G040 & Secretaries & 22 & 22 & & & & & & & & \\
\hline G050 & Typist and Word Processors & & & & & & & & & & \\
\hline G060 & Other General Admin, Secrelarial and Clerical Support & & $\stackrel{f}{t}$ & & & & & & & & \\
\hline
\end{tabular}


HNF-SP-1104 Rev 4

Exhibit: Program Average FTE Projections by COCS Categories

Ineludes Major Subcontractor and EntCo FTEs

Project Hanford Breakdown Structure/Title: Spent Nuclear Fuel Project

\begin{tabular}{|c|c|c|c|c|c|c|c|c|c|c|c|}
\hline COCS & Titie & FY 1997 & FY1998 & FY 1999 & FY 2000 & FY 2001 & FY 2002 & FY 2003 & FY $\overline{2004}$ & FY 2005 & FY 2006 \\
\hline Lo00 & Laborers and General Senvice Workers & 9 & 11 & 13 & 12 & 8 & & & & & \\
\hline L010 & Firefighters & & & & & & & & & . & \\
\hline L.020 & Food Service Workers & & & & & & & & & & \\
\hline L030 & Janitors and Cleaners & 3 & 3 & & & & & & & & \\
\hline LO40 & Laundry Workers & & 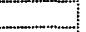 & & & & & & & & \\
\hline L050 & Handlers, Helpers and Laborers (General) & 1 & 2 & & & & & & & & \\
\hline L.060 & Handlers, Helpers and Laborers (Specialized) & & & & & & & & & & \\
\hline L070 & Light Vehicle Drivers & 5 & 5 & & & & & & & & \\
\hline L080 & Security Guards & 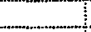 & 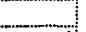 & & & & & & & & \\
\hline LOSO & Other Laborers and General Scrvices Workers & & 1 & & & & & & & & \\
\hline Mooo & Gen Mgrs, Exec, ist Line Suprv'sr \& Prog/Proj Mgrs & 70 & 67 & 63 & $50]$ & $20 \mid$ & & & & & \\
\hline M010 & First Line Supervisors & 25 & 22 & & & & & & & & \\
\hline M020 & General Managers and Exccutives & 24 & 24 & & & & & & & & \\
\hline M030 & Project and Program Managers & 6 & 6 & 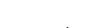 & & & & & & & \\
\hline $\mathrm{MO40}$ & Other Managers & 15 & 15 & & & & & & & & \\
\hline P000 & Professional Administrative \& Related Occupations & 47 & 45 & 42 & 38 & 201 & & & & & \\
\hline P010 & Accountants and Auditors & 10 & 9 & & & & & & & & \\
\hline $\mathrm{P020}$ & Architects & 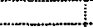 & 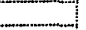 & . & & & & & & & \\
\hline P030 & Buyers, Procurement and Contracting Specialists & 5 & 5 & & & & & & & & \\
\hline P040 & Communications Specialists & & & & & & & & & & \\
\hline P050 & Compliance inspectors & & & & & & v & & & & $\cdot$ \\
\hline P060 & Computer Systems Analysts & & & & & & & . & & & \\
\hline P070 & Cost Estimators and Planners and Schedulers & 15 & 14 & & & & & & & & \\
\hline P080 & Health Physicists & 7 & 7 & & & & & - & & & \\
\hline P090 & Industrial Hygienists & 1 & 1 & & & & & & & & \\
\hline P100 & Lawyers & & & & & & & & & & \\
\hline P110 & Personnel and Labor Relations Specialists & & & & & & & . & & & \\
\hline P120 & Physicians & & & & & & & & & & \\
\hline P130 & Physician Assist, Nurses \& Oth Medical Supt Occup'tns & & & & & & & & & & \\
\hline P140 & Safeguards and Other Security Specialists & 2 & 2 & & & & & . & & & \\
\hline P150 & Trainers & 4 & 4 & & & & & & & & \\
\hline P160 & Technical Wrilers, and Edilors & 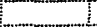 & $6 \cdot$ & & & · & & & & & \\
\hline P170 & Other Administrative \& Professional Other Occupations & 3 & 3 & & & & & & & & \\
\hline
\end{tabular}




\section{SPENT NUCLEAR FUEL PROJECT WBS 1.4.1}

HNF-SP-1104, Rev 4

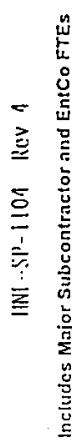

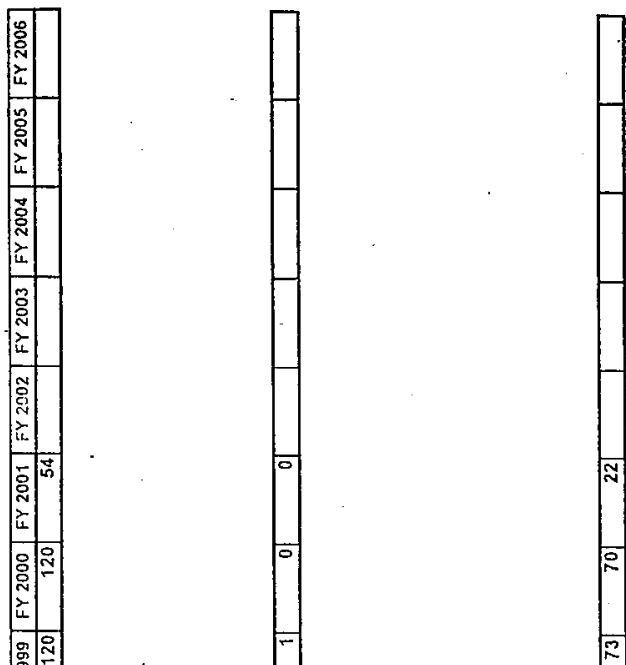

芯

尔

\begin{tabular}{|l|l|}
\hline & \\
\hline$\%$ & 0 \\
\hline 0
\end{tabular}

$\infty^{\infty}$

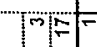

T.
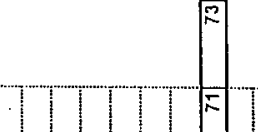

는

ํํำ

苋

o

O

तो

n

둥

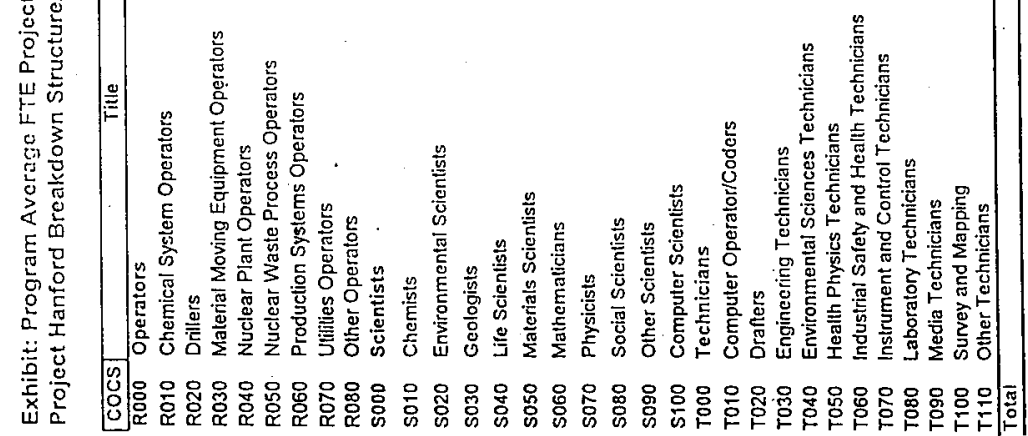

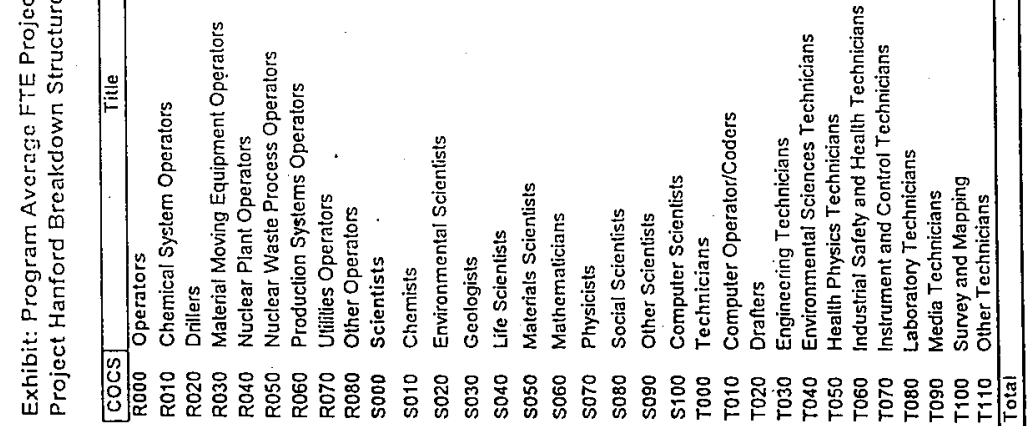

는

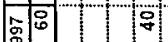

文

.

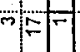

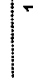

in

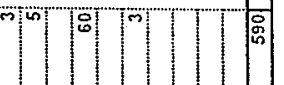




\section{SPENT NUCLEAR FUEL PROJECT \\ WBS 1.4.1}

HNF-SP-1104, REV 4

\subsection{ASSUMPTIONS AND RISKS}

\section{$5.1 \quad$ Assumptions}

\subsubsection{Technical}

Key SNF Project technical assumptions include:

- Characterization results -- The Project technical baseline was based on the assumption that the $\mathrm{K}$ West fuel was in a less degraded state than the $\mathrm{K}$ East fuel because the $\mathrm{K}$ West fuel had been treated with corrosion inhibitor and sealed. As a result, certain modifications to the $\mathrm{K}$ West Basin were eliminated from the Project scope that was assumed necessary for the $\mathrm{K}$ East Basin. The recent completion of the K West Basin "lift and look" campaign to validate this assumption revealed that the $\mathrm{K}$ West fuel was in a more degraded state than previously assumed. The cesium levels in the canisters were higher than expected and the amount of canister sludge was greater than assumed. Based on these results, the baseline assumption is modified to recognize that the $\mathrm{K}$ West fuel is roughly equivalent to the $\mathrm{K}$ East fuel, but no worse.

- $\quad$ RCRA Applicability -- The Resource Conservation and Recovery Act (RCRA) does not to apply to SNF.

- $\quad$ Sludge Disposition -- The current baseline assumes that K East Basin floor and canister sludge and $\mathrm{K}$ West Basin canister sludge will be managed as SNF while in the basins and will be transferred to the tank farms for management within the double-shell tank system after removal from the basins. Recent $\mathrm{K}$ East Basin sludge sampling has identified three locations where the PCB content exceeds the Toxic Substances Control Act (TSCA) limits. Resolution of technical issues associated with sludge disposition is included in the baseline. Any SNF sludge pretreatment requirements identified as a result of the resolution of either TSCA or technical issues will be incorporated through the baseline change control process.

- Integrated Water System effectiveness -- The Integrated Water System will be able to remove contaminants from the water as canisters are opened in the $\mathrm{K}$ West Basin. The IWTS will prevent excessive airborne contaminants and will prevent the ion exchange modules from becoming transuranic. Based on preliminary characterization data, $\mathrm{K}$ West basin canisters have an average of $2.14 \mathrm{Ci}$ cesium-137. It is assumed that $\mathrm{K}$ West basin 


\section{SPENT NUCLEAR FUEL PROJECT WBS 1.4.1}

HNF-SP-1 104, REV 4

has no more than 6.2 cubic meters of canister sludge and $\mathrm{K}$ East basin has no more than 10.6 cubic meters. Excess water removed from the basins will be transferred to the 200 Area Effluent Treatment Facility. No polychlorinated biphenyls (PCBs) are assumed to be present in the canister sludge.

- $\quad$ CVD Water Management -- The original baseline defined a process in which water extracted from MCOs during cold vacuum drying would be disposed of through the 200 Area Effluent Treatment Facility (ETF). It has subsequently been determined that the CVD process results in effluent which will not meet the acceptance criteria for the 200 Area ETF and that an alternate strategy for water management is required. Based on an analysis of alternatives, the baseline has been changed to require the return of CVD process water to the K West Basin IWTS through the lines flowing directly to the ion exchange system.

- $\quad$ Qffice of Civilian Radioactive Waste Management (OCRWM) QA Requirements -Analysis of changes to the existing SNF Project QA program and associated costs has been completed. However, the identified changes have not been finalized for implementation. The OCRWM requirements will be implemented on the project when SD-SNF-QAPP-004, Rev 1 is submitted to RL by FDH and approved by RL. Rough order of magnitude funding to implement the OCRWM requirements has been identified in this MYWP. Changes to both cost baselines may be necessary when the QAPP is approved.

- $\quad$ CSB/SNF Project scope -- The Canister Storage Building is being designed for SNF storage with an option to store high-level waste canisters; no plans are being made for storage of other materials, such as $\mathrm{Cs} / \mathrm{Sr}$ capsules.

- $\quad$ Sealed MCO Staging -- The SNF Project design was based on the strategy that the MultiCanister Overpacks. (MCO) would be vented to the Canister Storage Building (CSB) tubes during the staging period which precedes hot conditioning. A key management decision has been made to change from a vented to a sealed MCO during staging. The decision to seal MCOs impacts the technical, schedule, and cost baselines, design analyses, and some specific building/equipment design features. In the vented condition, hydrogen would be vented continuously from the MCO to the CSB storage tubes where it would have been periodically purged. With the sealed configuration, the hydrogen will be contained within the MCO and purged as an initial step prior to hot conditioning. Specific impacts include changes to CSB storage tubes, storage tube plugs, $\mathrm{MCO}$ 


\section{SPENT NUCLEAR FUEL PROJECT \\ WBS 1.4.1}

HNF-SP-1104, REV 4

Handling Machine and instrumentation system, and design or operational impacts in the Hot Conditioning System (HCS) associated with MCO overpressure blowdown.

- NRC Equivalency (tornado hardening) -- The Project has accepted NRC equivalency requirements for the Project to establish a degree of safety equivalence with NRClicensed facilities. This requires that the CSB and Cold Vacuum Drying (CVD) facility be designed and constructed to withstand tornado loadings which are not included in DOE design requirements. Primary impacts are in design and construction of these facilities, especially for the CSB operating shelter and service building and the CVD walls and roof.

- $\quad$ HCS pressure management -- An enabling assumption for the current HCS design is that the rupture disk will not actuate while an MCO is in the HCS process pit. Fuel characterization studies and computer modeling of fuel reactions are being conducted to validate this assumption. Until these studies demonstrate that this assumption is valid it will remain as an open issue.

- Interim storage period -- K Basins SNF and other Hanford SNF will be interim stored in the CSB or in the 200 Area ISA, pending decisions on final disposition. The design life of the CSB is 40 years. Steps have been taken to allow a potential increase in service life of interim storage facilities beyond 40 years to minimize the impact of uncertainties in the availability of a permanent repository.

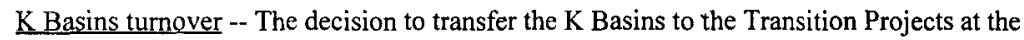
conclusion of the SNF Project changed the post-2001 responsibility for $\mathrm{K}$ Basins water and facilities from the SNF Project to the Transition Projects. A contractor-level Memorandum of Understanding has proposed programmatic interfaces at turnover which may result in some differences to the SNF Project baseline to improve the efficiency of turnover between the PHMC major subcontractors. The Revision 0 MYWP baseline reflects the transfer of the basin from the SNF Project to the Transition Projects. Any changes proposed by the PHMC will be submitted to RL for approval through the appropriate change control process.

- Facilities Transition Projects interface: 300 Area facilities -- Budget and schedule within Facilities Transition Projects for packaging and removal of 300 Area Light Water Reactor (LWR) fuel is currently being modified. Schedules, budgets, and technical strategy are being re-evaluated within the SNF Project and will likely require changes to Project baselines (which currently includes LWR fuel being transferred to the 400 Area Interim 


\section{SPENT NUCLEAR FUEL PROJECT WBS 1.4.1}

HNF-SP-1 104, REV 4

Storage Area (ISA) prior to transfer to the 200 Area ISA when it is available). Anticipated changes include not transferring LWR fuel to the 400 Area ISA prior to its final move to the 200 Area Interim Storage Area and possible storage of fuel within the CSB.

- $\quad$ HSTB Database Changes -- Changes to the SNF Project technical baseline are anticipated due to changes to the structure of baseline documentation as reflected in the HSTB data (Section 1, Exhibit 1A) and the life cycle requirements that the SNF Project facilities and sub-projects must meet. The technical baseline will be revised to incorporate these and other changes after the July 1997 revision of the HSTB database.

- $\quad$ FFTF fuel disposition -- Sodium-bonded FFTF fuel will be transferred offsite for treatment. Non-self protecting fuel will be stored in the Plutonium Finishing Plant if necessary for safeguards purposes pending decisions on final disposition. 


\section{SPENT NUCLEAR FUEL PROJECT \\ WBS 1.4.1}

HNF-SP-1 104, REV 4

\subsubsection{Cost/Schedule}

The following order of magnitude costs were included in the MYWP from FY 1997 to FY 2001.:

- $\$ 3.0 \mathrm{M}$ was used as an order of magnitude estimate for KE Basin Tritium Level Reduction.

- $\$ .5 \mathrm{M}$ was used as an order of magnitude estimate for QA Program/OCRWM to cover cost associated with outstanding QA Program compliance issues and incorporation of future OCRWM requirements.

- $\$ 2.5 \mathrm{M}$ was used as an order of magnitude estimate for Sludge Disposal to cover additional scope arising from Regulatory requirements regarding PCB management and disposal and Task Waste Remediation System (TWRS) requirements for preprocessing of sludge prior to shipment to the Tank Farms.

- $\quad \$ 3.0 \mathrm{M}$ was used as an order of magnitude estimate for $\mathrm{KW}$ Integrated Water Treatment System (IWTS) design. This entails replicating the KE design in KW. The estimate was based on the current estimates for KE IWTS.

- $\quad \$ 1.0 \mathrm{M}$ was used as an order of magnitude estimate for IWTS (Cold Vacuum Drying Offload Station). Constructing a CVD offload station where CVD process return water is returned to the basin IWTS in the lines flowing directly to the ion exchange media. 
This page intentionally left blank. 


\section{SPENT NUCLEAR FUEL PROJECT \\ WBS 1.4.1}

HNF-SP-1104, Rev 4

\subsection{RISKS}

\subsubsection{Technical}

- $\quad$ KE Basin Tritium Level Reduction -- Reduction of tritium levels in K East Basin may be required prior to final water removal to provide maximum protection to the environment and to comply with the re-negotiated Tri-Party Agreement (TPA). This will be determined when agreement is reached with regulators. \$9M was requested in FY 2001 but is not part of this Baseline.

- $\quad \mathrm{MCO}$ detonation -- If analysis or testing cannot show that the current design precludes hydrogen/oxygen detonation within MCOs, additional process steps and equipment may have to be added to confirm $\mathrm{MCO}$ oxygen generation rates at a process station or in the CSB.

- $\quad$ Recejpt of pressurized $\mathrm{MCO}$ in HCS -- The current design basis assumes that actuation of the MCO pressure relief system does not occur in the HCS. If analyses, tests, or administrative controls cannot preclude $\mathrm{MCO}$ pressurization during staging, a safety class inerting system would have to be added to the HCS to accommodate a pressurized MCO. As an alternate, the CSB service station could be upgraded to vent pressurized MCOs prior to transfer to the HCS. The magnitude of change for either option could exceed Revision 0 MYWP budgets.

- Beyond-design-basis accident analysis - The fast track schedule associated with the SNF Project has dictated the need for process development concurrent with the detailed design and safety analysis activities. As a result, the beyond-design-basis accident analysis is yet to be performed as part of the final Safety Analysis Report preparation. If this analysis invalidates any of the key enabling assumptions, an assessment of the required facility changes will be made.

- $\quad$ N Basin fuel chips -- The recent discovery of fuel chips in the N Basin may require SNF Project work scope changes. Because the Environmental Restoration Management Contractor and it's subcontractors do not have nuclear material accountability within their authorized workscope, the PHMC may need to take responsibility for the material. The current baseline assumes that the SNF Project does not receive fuel chips from $\mathrm{N}$ Basin. Additional workscope, cost, and schedule may need to be added to accommodate packaging, transport, and storage of the $\mathrm{N}$ Basin if it becomes the 


\section{SPENT NUCLEAR FUEL PROJECT \\ WBS 1.4.1}

HNF-SP-1104, Rev 4

responsibility of the SNF Project. Change Control will be implemented prior to addition of this workscope.

- South load-out pit accident analysis - Assumptions currently contained in the transport cask drop accident analysis are undergoing internal and external review. Any unresolved concerns related to these assumptions may dictate a need for mitigating design features currently not within the Project scope.

- Current sludge and liquid/gas -- characterization data and "lift and look" estimations indicate that the problems associated with the K-West Integrated Water Treatment System are significantly more difficult than previously assumed. These include higher than anticipated sludge volumes and $\mathrm{Cs}-137$ concentrations. Issues in $\mathrm{KE}$ include weasel pit heat generation, criticality and hydrogen generation.

- Ion Exchange Column (IXC) Disposition -- Funds are budgeted in FY 1998 (\$398K) and $2000(\$ 5971 \mathrm{~K})$ for disposition of 33 transuranic (TRU)-contaminated and 6 low-level IXCs stored in the K West Basin Chlorine Vault and 6 TRU-contaminated IXCs stored in the K East Basin. The TRU contamination, the high radiation fields (up to $100 \mathrm{R}$ ), and the hydrogen generated by the IXCs pose challenges that require the development of special packaging for transport and storage. The funds budgeted were based on estimates with minimal information about the packaging requirements. Additional costs and workscope may be encountered as development and fabrication of the packages and the disposition of the IXCs proceed. 


\section{SPENT NUCLEAR FUEL PROJECT \\ WBS 1.4.1}

HNF-SP-1 104, Rev 4

\subsubsection{Cost \& Schedule}

Contingency breakdown from FY 1997 to FY 2001:

- $\quad \mathrm{K}$ West Sludge Disposition -- Current Baseline is to transfer K West floor sludge to Transition Projects, if during fuel removal operations it is found that the K West sludge needs to be handled as transuranic, it will be incorporated into the Baseline. $\$ 4 \mathrm{M}$ has been allocated as contingency.

- Expense -- 5.2\% or $\$ 11.8 \mathrm{M}$ has been allocated for Expense Contingency to cover design evolutions and process improvements that will be found as part of the Cold Test Facility and Fuel Movement Operations. Non-scope related cost impacts.

- Operations -- $5.9 \%$ or $\$ 14.5 \mathrm{M}$ has been allocated for Operations Contingency due to the first of a kind operations.

- $\quad$ Line Item -- $3.5 \%$ or $\$ 3.1 \mathrm{M}$ has been allocated for Line Item Contingency for non-scope related impacts.

- $\quad$ FRS $--6.6 \%$ or $\$ 1.1 \mathrm{M}$ has been allocated for FRS Contingency for non-scope related impacts.

- $\quad$ MHM $-2.9 \%$ or $\$ .2 \mathrm{M}$ has been allocated for MHM Contingency for non-scope related impacts. 


\section{SPENT NUCLEAR FUEL PROJECT}

WBS 1.4.1

HNF-SP-1 104, Rev 4

This page intentionally left blank. 